\title{
LAWRENCE BERKELEY LABORATORY INSTITUTIONAL PLAN FY 1995-2000
}

December 1994

Lawrence Berkeley Laboratory

University of California

Berkeley, California 94720

Prepared for the U.S. Department of Energy under Contract No. DE-AC03-76SF00098 DISTRIBUTION OF THIS DOCUMENT IS UNLMITED 
89 reycles paper 


\section{DISCLAIMER}

This report was prepared as an account of work sponsored by an agency of the United States Government. Neither the United States Government nor any agency thereof, nor any of their employees, make any warranty, express or implied, or assumes any legal liability or responsibility for the accuracy, completeness, or usefulness of any information, apparatus, product, or process disclosed, or represents that its use would not infringe privately owned rights. Reference herein to any specific commercial product, process, or service by trade name, trademark, manufacturer, or otherwise does not necessarily constitute or imply its endorsement, recommendation, or favoring by the United States Government or any agency thereof. The views and opinions of authors expressed herein do not necessarily state or reflect those of the United States Government or any agency thereof. 


\section{DISCLAIMER}

Portions of this document may be illegible in electronic image products. Images are produced from the best available original document. 


\section{PREFACE}

The Institutional Plan provides an overview of the Lawrence Berkeley Laboratory mission and strategic plan. For FY 1995-2000, the Institutional Plan reflects significant revisions based on the core business areas and critical success factors of the Department of Energy's Strategic Plan, "Fueling a Competitive Economy," and the Laboratory's strategic planning process.

The Strategic Plan section identifies long-range conditions that will influence the Laboratory, as well as potential research trends and management implications. The Core Business Areas section identifies by subsections of Science and Technology, Energy Resources, Environmental Quality, and Industrial Competitiveness potential new research programs that represent major long-term opportunities for the Laboratory, and the resources required for their implementation. It also summarizes current programs and potential changes in research program activity. The Critical Success Factors section reviews human resources; environment, safety, and health; management practices (with subsections on quality assurance and self assessment, administrative management, information resources management, and site and facilities); and communications and trust. The Resource Projections are estimates of required budgetary authority for the Laboratory's ongoing research programs.

The Institutional Plan is a management report for integration with the Department of Energy's strategic planning activities, developed through an annual planning process. The plan identifies technical and administrative directions in the context of the national energy policy and research needs and the Department of Energy's program planning initiatives. Preparation of the plan is coordinated by the Office for Planning and Communications from information contributed by the Laboratory's scientific and support divisions. 


\section{TABLE OF CONTENTS}

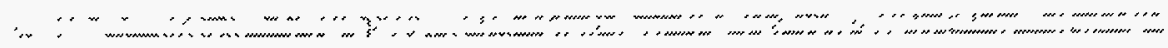

PREFACE

I. DIRECTOR'S STATEMENT

II. THE LABORATORY MISSION

Core Competencies and Foundations

Division Strengths

III. LABORATORY STRATEGIC PLAN

LBL'S Vision and Strategic Objectives

Situation Analysis

Laboratory Constraints

Objectives and Strategies

Strategic Actions

Management Issues

IV. CORE BUSINESS AREAS

Science and Technology

ALS Beamlines Initiative

Combustion Dy'namics Initiative

Structural Biology Initiative

Genome Sequencing Initiative

Transgenic Initiative

Atomic-Scale Synthesis of Advanced Materials Initiative

Electron Beam Microcharacterization Facility Initiative

B Factory at Stanford Linear Accelerator Initiative

Large Hadron Collider Initiative

Relativistic Heavy-Ion Collider Initiative

National Information Infrastructure: Networking Initiative 
Energy Resources 4-38

Heavy-Ion Fusion Driver Initiative 4-38

Energy Efficiency for the Climate Change Action

Plan Initiative $\quad$ 4-39

Advanced Petroleum Technologies Alliance Initiative 4-41

Environmental Quality 4-47

Assisting Development of Energy Practices and

Technologies Initiative 4-47

Energy and Environmental Technology Strategic Initiative $\quad$ 4-49

Advanced Risk-Based Environmental Technologies and

Remediation Initiative 4-50

California Environmental Enterprise Initiative 4-52

Industrial Competitiveness $\quad$ 4-57

Advanced Lithography Initiative 4-58

Fabrication of Microelectromechanical Systems, Using Deep

X-ray Lithography Initiative 4-59

AMTEX: The AMerican TEXtile Partnership Initiative 4-61

Northern California Defense Conversion Initiative 4-63

V. CRITICAL SUCCESS FACTORS 5-1

Human Resources 5-1

Environment, Safety, and Health 5-12

Management Practices $\quad 5-22$

Communications and Trust $\quad 5-43$

VI. RESOURCE PROJECTIONS 6-1

VII. ACKNOWLEDGEMENTS $7-1$

VIII. ACRONYMS AND ABBREVIATIONS 


\section{Director's Statement}

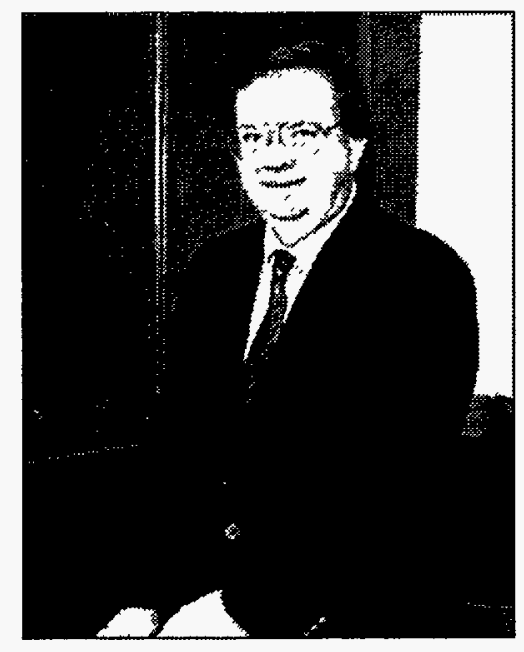

Charles V. Shank, LBL Director
Through LBL's Institutional Plan, we combine our strategic planning with that of the Department of Energy to address our nation's future. The benefits of the national laboratory system are being realized through our strengths in the basic sciences and new research and technological partnerships with industry, academia, and other national laboratories. These partnerships enrich our understanding of the world, create value for the economy, enhance education and contribute to our larger community.

The Lawrence Berkeley Laboratory (LBL) solves science and technology problems of national significance by making available the advanced scientific capabilities of a multiprogram national laboratory. For more than 60 years, LBL has maintained a tradition of outstanding research in advanced materials, biosciences, energy efficiency, earth and environmental sciences, and the use of particle beams and detectors to understand the fundamental properties of matter. Today, our partnerships with industry and universities are creating value for the economy, improving the environment and health care, and enhancing the education of our citizens. We are setting a scientific agenda for the next ten years that reflects both the critical technology needs of the nation and the Laboratory's ability to contribute to those needs.

In 1992, I initiated Vision 2000, the start of a major strategic planning effort at LBL. The purpose of this effort was to define the Laboratory's major objectives and provide LBL, its employees, its partners, and the Department of Energy with a clear statement of our plans and goals for the future. Vision 2000 affirms basic research as our foundation and establishes a commitment to reach out and forge partnerships, to develop our resources and our people to create value for the economy, to enhance education, and to contribute to our community. This FY 1995-2000 Institutional Plan describes progress towards our vision and brings focus to these efforts by illustrating how its core competencies and the resulting business lines and 
critical success factors make us a key element of the national laboratory system.

LBL plans to strengthen its user facilities that are already serving national research needs. The Advanced Light Source (ALS) was completed on schedule, within budget, and with performance exceeding project specifications. This has begun an era of scientific programs at the ALS that is at the forefront of research in materials, surfaces, interfaces, chemical dynamics, and biosciences, with partners drawn from the national scientific community. Completion of the Advanced Light Source Beamlines Initiative is a crucial element of the Laboratory's strategic plan. To further enhance the potential of the ALS, the Laboratory's Structural Biology Initiative will also add user capabilities for studies in biological microscopy, crystallography, and spectroscopy. LBL continues to emphasize the value of scientific partnerships through such collaborations as the Combustion Dynamics Initiative. This program will combine the capabilities of two national laboratories-Sandia and LBL-and LBL's educational ties with the University of California to research the characteristics and potential improvement in efficiency of fossil fuel combustion and emission.

Our advances in biosciences hold special promise for improvements in health and the environment, and for U.S. industry. LBL's Human Genome Center, with its ability to deliver new levels of sequencing productivity, and expanding activity in structural biology research offer prospects for a much deeper understanding of the environmental and genetic causes of health risks and disease. This research also offers advances in the area of molecular structure that will eventually allow the manufacture of custom-tailored pharmaceuticals, and the development of microbioal systems to eliminate contaminants from the soil. The information generated at the LBL Human Genome Center has already contributed to the development of improved methods for screening genetic abnormalities, locating cancer genes, and diagnosing solid tumors. Further advances with our new sequencing facility hold great promise for further improving sequencing, with benefits to our many research partners.

In materials research, the Laboratory is addressing pressing national needs for establishing the molecular and atomic basis for materials design, characterization, and processing. We are directing our capabilities to those areas where our greatest strengths lie: atomically structured materials and devices, bioorganic synthesisceramics and alloys, heterostructures, and superconducting and magnetic materials. The area of advanced materials has also been identified as an "enabling technology" for other critical technologies, including biotechnology, transportation, manufacturing, energy and environment, and information and communication.

To meet the nation's need for technologies for energy supply, the Laboratory continues at the forefront of inertial confinement fusion research. To this end, we will continue to develop heavy-ion accelerators as fusion energy drivers. They offer the most promising prospect for efficient and reliable pellet ignition for civilian power production during the middle of the 21 st century. LBL also continues to pioneer advances, including development of electromagnetic and seismic methods, for imaging subsurface resources such as oil and gas. 
To address the pressing national need for improved energy efficiency, our leadership in advanced building technologies and electrochemical research is being applied to new lighting systems and superwindow technology, and to restructuring the way innovative building systems can be incorporated in residential and commercial design. This program advances from our accomplishments in developing building and appliance codes and standards, low-emissivity windows, and high-frequency fluorescent lighting, as examples. In addition, we have formed new partnerships with industry for advanced battery development and combustion research. We serve as an international resource for energy efficiency technologies and provide technical assistance on energy supply and demand to developing nations.

The Laboratory is also well positioned for leadership in environmental remediation technologies, with research programs in improved characterization methods, development of predictive models and risk-assessment methodologies, development of techniques to isolate and clean up sites contaminated by radioactive materials and toxic chemical wastes (including bioremediation techniques), and R\&D on waste minimization and effective means of waste disposal. For example, we have developed new polymer gel technology for immobilizing subsurface contaminants in place, with the technology currently being tested at leaking waste tanks at Hanford.

LBL will maintain its historic strengths in high-energy and nuclear physics, providing leadership for the B Factory at the Stanford Linear Accelerator Center (SLAC) and the STAR detector at the Relativistic Heavy lon Collider (RHIC). The Laboratory's competencies also provide an outstanding opportunity to help design and fabricate key detector and accelerator systems at the Large Hadron Collider in Europe.

LBL's continuing commitment to its people has been reflected in institutional changes. The Office of Work Force Diversity was established to ensure an ethic that fully respects diversity and encourages excellence. Our commitment to the environment continues to be extremely important in our day-to-day operations. We have incorporated major changes in our response to environmental and safety concerns through a continuous improvement program that ensures that our work is carried out with full regard for the environment and the health and safety of our employees and neighbors.

To be fully successful in adding value to the nation, LBL is committed to its strategy of partnership with industry, universities, and other federal agencies. LBL's partnerships bridge the gap between research and development and manufacturing industries, including electronics, textiles, pharmaceuticals, energy utilities, transportation, and construction. Our university partnerships, building on our historic alliances with the University of California, position us to contribute to programs at hundreds of universities nationwide. Our partnerships with other government agencies, including the National Institutes of Health, the National Aeronautics and Space Administration, and the Environmental Protection Agency, add value for all our citizens, as is fully appropriate for a DOE multiprogram national laboratory. 
As LBL charts its course into the 21 st century, we will continue to refine our strategic objectives and to articulate clearly this institution's contribution to the Department of Energy and its partners. A focused communications program is being directed towards improving the national understanding of our research contributions and strategic directions. We are strengthening our partnerships with industry, other government agencies, and educational institutions to better serve national needs. Our ultimate goal is to see that our people and our technological resources continue to serve as a unique and valuable resource for the Department of Energy and the nation.

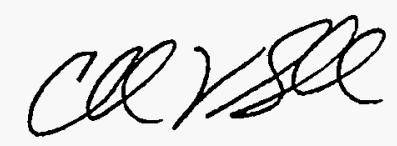

Charles V. Shank

Director 
The Lawrence Berkeley Laboratory is a multiprogram national research facility operated by the University of California for the Department of Energy (DOE). Its fundamental mission is to provide national scientific leadership and technological innovation to support the DOE's objectives. LBL's mission addresses four distinct goals:

- To perform leading multidisciplinary research in the energy sciences, general sciences, and biosciences in a manner that ensures employee and public safety and protection of the environment.

- To develop and operate unique national experimental facilities that are available to qualified investigators: the Advanced Light Source, National Center for Electron Microscopy, 88-Inch Cyclotron, and National Tritium Labeling Facility.

- To educate and train future generations of scientists and engineers to promote national science and education goals.

- To transfer knowledge and technological innovations and foster productive relationships between LBL research programs, universities, and industry to promote national economic competitiveness.

\section{¿CORE COMPETENCIES AND FOUNDATIONS}

The ability of the Laboratory to advance its mission depends upon its "core competencies." These are an integration of research disciplines, personnel, skills, technologies, and facilities that produces results of value to our sponsors and all customers. The core competencies can be applied to rapidly changing national needs and new research problems while, at the same time, undergoing evolution themselves. 
Underlying many of our core competencies in specific technical areas are fundamental capabilities or "foundations." LBL has identified seven core competencies and four foundations as follows.

\section{Core Competencies}

- Bioscience and Biotechnology: Structural biology; genome research; bioinstrumentation; molecular cytogenetics; medical imaging; biology of human diseases; biomolecular design.

- Particle and Photon Beams: Analysis and design of accelerators; beam dynamics; high-brightness ion, electron, and photon sources; advanced magnet design and R\&D; high-frequency rf technology; $x$-ray optics and lithography; induction linacs and neutral beams for fusion energy.

- Characterization and Synthesis of Materials: Advanced spectroscopies and microscopies based on photons, electrons, and scanning probes; ceramics; alloys; heterostructures; superconducting, magnetic, and atomically structured materials; bioorganic synthesis.

- Advanced Technologies for Energy Supply and Energy Efficiency: Subsurface resources and processes; building technologies; electrochemistry; fossil fuel technologies; energy analysis.

- Chemical Dynamics, Catalysis, and Surface Science: Reaction dynamics; photochemistry of molecules and free radicals; surface structures and functions; heterogeneous, homogeneous, and enzymatic catalysis.

- Advanced Detector Systems: Major detectors for high-energy physics, nuclear science, and astrophysics; scientific conception and project leadership; advances in particle and photon detection; implementation of new concepts in detector technology.

- Environmental Assessment and Remediation. Advanced instrumentation and methods for environmental characterization and monitoring; human health and ecological risk assessment; indoor air quality; subsurface remediation of contaminants; geologic isolation of highlevel nuclear waste; actinide chemistry.

\section{Foundations}

- National Research Facilities: Advanced Light Source; National Center for Electron Microscopy; 88-Inch Cyclotron and Gammasphere; National Tritium Labeling Facility.

- Computation and Information Management: High-speed networking and distributed computing; processing and analysis of scientific images; data-acquisition and analysis systems; scientific information systems; database technology.

- Engineering Design and Fabrication Technologies: Custom integrated circuits; integrated accelerator systems; superconducting magnet assemblies; insertion devices for synchrotron radiation; large-volume semiconductor detector technology; laboratory automation; advanced CAD/CAM facilities for large systems; facilities for materials processing and fabrication. 
- Education of Future Scientists and Engineers: Undergraduate, graduate, postdoctoral, and faculty involvement in scientific and engineering research through close ties with the University of California system; educational programs for elementary schools, high schools, and colleges.

\section{DIVISION STRENGTHS}

LBL benefits from its close working relationship with the University of California at Berkeley, as well as other universities, laboratories, and industrial institutions. As indicated in the organization chart, the Laboratory is structured to integrate these relationships with its mission in the most effective way possible. The core research strengths of each of the LBL divisions are as follows:

\section{Energy Sciences Divisions}

- Chemical Sciences — chemical physics and the dynamics of chemical reactions; structure and reactivity of transient species; electron spectroscopy; surface chemistry and catalysis; electrochemistry; chemistry of the actinide elements and their relationship to environmental issues; atomic physics.

- Earth Sciences-structure, composition, and dynamics of the earth's subsurface; geophysical imaging methods; chemical and physical transport in geologic systems; isotopic geochemistry; physicochemical process investigations.

- Energy and Environment-building energy efficiency; environmental effects of technology; energy storage and distribution; fossil-energy conversion; industry, transportation, and utility energy use; national and international energy policy studies.

- Materials Sciences_-advanced ceramic, metallic, polymeric, magnetic, biological, and semi- and superconducting materials for catalytic, electronic, optical, magnetic, structural, and specialty applications; exploration of low-dimensional materials; development and use of instrumentation including spectroscopies, electron microscopy, $x$-ray optics, nuclear magnetic resonance, and analytical tools for ultrafast processes and surface analysis.

\section{General Sciences Divisions}

- Accelerator and Fusion Research-fundamental accelerator physics research; accelerator design and operation; advanced accelerator technology development for high-energy and nuclear physics; accelerator and beam physics research for heavy-ion fusion; beam and plasma tools for materials sciences and engineering; operation of the Advanced Light Source.

- Nuclear Science-relativistic heavy-ion physics; medium- and lowenergy nuclear physics; nuclear structure; nuclear theory; nuclear astrophysics; weak interactions; nuclear chemistry; studies of transuranium elements; nuclear-data evaluation; detector development; operation of the 88-Inch Cyclotron. 
- Physics-experimental and theoretical particle physics; advanced detector development; particle database for the high-energy physics community; astrophysics; applied mathematics; innovative education programs for high schools.

\section{Biosciences Divisions}

- Life Sciences-gene expression; molecular genetics and human genome studies; cellular differentiation and carcinogenesis; hematopoiesis; macromolecular structure; DNA repair and recombination; diagnostic and functional imaging; and radiation biology.

- Structural Biology-structural and molecular biology of nucleic acids and proteins; genetics and mechanisms of photosynthesis; photochemistry; and mechanisms of mutagenesis.

\section{Resources and Operations Divisions}

- Engineering-engineering design, planning, and concept development; advanced accelerator components; electronic and mechanical instrumentation; scientific applications software development; laboratory automation; fabrication of detectors and experimental systems; shops and technical support for scientific programs and research facilities.

- Environment, Health and Safety-technical support for safety and environmental protection; radiation associated with accelerator technology; advanced dosimeters; dispersion of radionuclides; waste management.

- Information and Computing Sciences-advanced software engineering; information management; network development; scientific imaging and visualization tools; computation tools for the human genome project; biostatistics; agile manufacturing. 


\section{LAWRENCE BERKELEY LABORATORY • UNIVERSITY OF CALIFORNIA}

ASSOCIATE DIRECTOR AT LARGE G.T. SEABORG

\section{LABORATORY DIRECTOR C.V. SHANK \\ DEPUTY DIRECTORS KH. BERKNER P.J. ODDONE}

LABORATORY COUNSEL G.R. WOODS

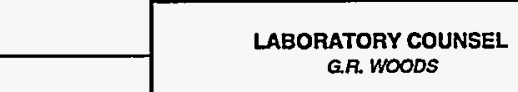
H. REED

INTERNAL AUDT R.T. TUFFNEU, Acting

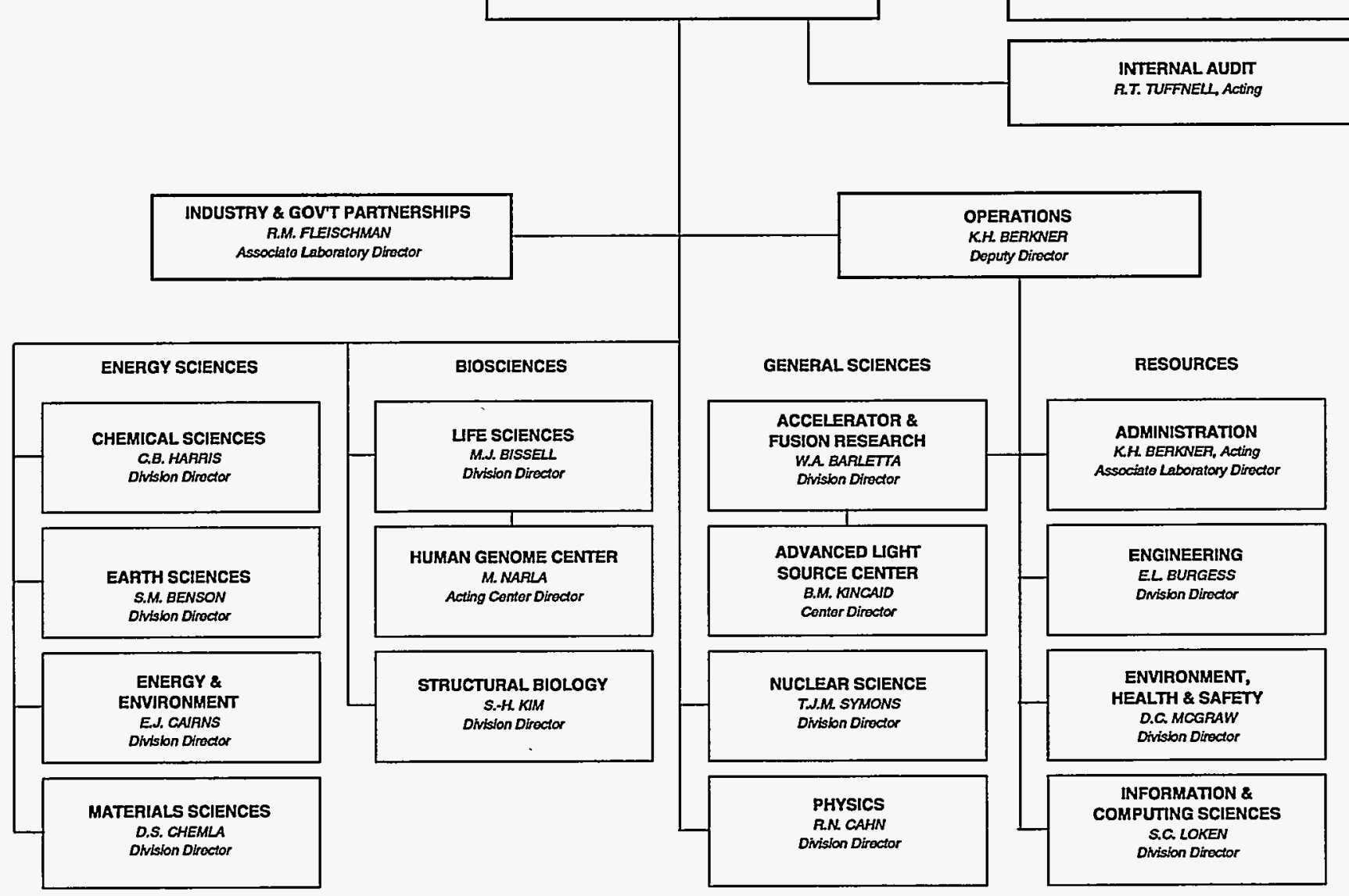

LBL organization chart. 


\section{III}

\section{LABORATORY STRATEGIC PLAN}

\section{U. LBL'S VISION AND STRATEGIC OBJECTIVES}

The strategy outlined in this section addresses the R\&D environment of the national laboratories that affects the LBL's research priorities and the allocation of its resources. LBL's Vision 2000 establishes the overarching goals of the Laboratory's strategic planning effort. It focuses on a commitment to reaching out and forging new partnerships with industry, academia, and other national laboratories to create value for the economy, enhance education, and contribute to the community. The four major goals of Vision 2000 are as follows:

Distinguish ourselves as a premier DOE multiprogram national laboratory by performing research of the highest scientific quality. Build on our educational and technical resources to gain a competitive advantage for addressing problems of national significance and advancing the mission of the DOE.

Create value for the economy, enhance education, and contribute to the community through partnerships with industry, universities, and other laboratories.

Make LBL the location of choice for facilities and programs. Our operational, administrative, and technical resources will integrate seamlessly with the research and engineering programs to make an LBL that works. All of our activities will be conducted with full regard for the environment, health, and safety.

Commit to developing our people to their fullest potential. We value and seek diversity in our work force. We will create an environment that respects the individual, en- 
courages leadership, stimulates innovation, fosters integrity, and demands excellence.

To achieve these goals, we must understand the context of our research programs within the framework of DOE, other national laboratories, and the nation, and must incorporate this information into our strategic planning effort.

\section{$\therefore$ SITUATION ANALYSIS}

LBL is dedicated to multiprogram energy research that addresses vital energy, economic, and educational interests. The world has entered an era fundamentally different from the one that motivated the establishment of the national laboratories. Pressing economic conditions in concert with the end of the Cold War have caused a reevaluation of national research priorities within the larger world community. U.S. global economic competitiveness and the preeminence of U.S. science and engineering have emerged as important federal goals, along with new imperatives for improving government and addressing health, safety, and environmental concerns. National laboratories will be called upon to function as an efficient and effective system that supports DOE's strategic business lines for maintaining the foundations of science and technology, improving energy efficiency and supply, protecting the environment, and strengthening competitive advantage. We will be expected to carry out these missions in a manner that incorporates the highest standards for health, safety, and environmental protection.

\section{Science and Technology}

- Structural biology, biotechnology, and the human genome. These efforts include research underlying the development of new medical treatments and commercial products, including activities exploiting such modern techniques as genetic engineering and the use of monoclonal antibodies and structural studies based on x-ray diffraction and spectroscopy, neutron scattering, and magnetic resonance imaging. They demand continuing development of instrumentation and computation, and underpin the nation's biotechnology industry.

- Materials for technological applications. R\&D efforts are needed for the development of superconducting, catalytic, polymeric, magnetic, biological, and tribiological materials and materials for electronic applications that have long-term economic benefit. Cross-cutting research efforts include those aimed at developing nanometer-scale artificially structured materials; materials for harsh chemical environments and high-radiation applications; and techniques for synthesis, processing, and characterization. This demands continuous development of advanced facilities and instrumentation.

- Scientific foundations. The universe, matter and its interactions, and basic science as a foundation for future technology development. This effort includes research in high-energy and nuclear physics, planetary studies, astronomy, and astrophysics. Such research demands continuing development of investigative tools, including advanced accelerator/ detector facilities and space technologies. Basic science studies span 
the scientific spectrum, including materials, chemical, geosciences, and life sciences research. Broadly based fundamental research is the ultimate source of long-term technological advancement of DOE's mission.

\section{Economic Competitiveness}

- High-performance computation and information management. These are efforts focused primarily in the areas of massively parallel processing and high-performance, network-mediated collaborative computing. They demand continuous development of both hardware and software as well as telecommunication technology, and are vital to the nation's economic future.

- Advanced manufacturing and industrial processes. These efforts are broadly aimed at developing and applying technology-driven techniques and scientific insights to the improvement of manufacturing methods and industrial processes. Examples include the development of advanced robotics, the application of surface science research, studies of heterogeneous catalysis to crucial processes in the oil and chemical industries, and a collaboration of the textile industry with the national laboratories to explore advanced manufacturing techniques.

\section{Energy Efficiency and Supply}

- Energy supply and resource development technology. Energy supply research is needed to improve the performance, economics, and environmental effects of technologies such as solar energy systems (e.g., photovoltaic cells, biomass combustion and conversion), coalburning systems, fission reactors, and fusion-based energy sources. Sustainable resources, including fusion energy, are key to the nation's R\&D program. Resource development technology is needed to ensure the optimal, environmentally safe exploitation of resources, and geosciences research to allow accurate prediction of natural disasters such as earthquakes and volcanic eruptions. Required activities include the development of better techniques for characterization, resource evaluation, and prediction of natural and induced processes.

- Technologies to increase the efficiency of energy use. These include fundamental research in chemical and combustion dynamics as well as development of more energy-efficient building technologies, industrial processes, and transportation systems. They encompass research on energy-efficient windows; lighting; heating, ventilating, and air-conditioning (HVAC) systems for buildings; and the development of vehicles using fuels derived from natural gas and biomass; as well as other advanced air and surface transportation systems.

\section{Environmental Quality and Remediation}

- Ecological, climatological and medical risks of energy use and mitigation of environmental contamination. These needs include biological and medical research on plant and animal response to pollution and climate modeling research. Solving critical problems in global warming demands advances in computation, including new data manage- 
ment tools, parallel algorithms, speed of processing, and size of processors and storage. Environmental contamination mitigation requires research on improved characterization methods, development of predictive models and risk-assessment methodologies, development of techniques to isolate and clean up sites contaminated by toxic chemical wastes and radioactive materials (including bioremediation techniques), and R\&D on waste minimization and disposal.

- Environmental and Energy Policy. This research is needed to strengthen U.S. policies for energy use and environmental protection, in support of DOE's mission. This includes development of improved methods for analyzing policies to increase energy efficiency, assessing alternative strategies for R\&D investment and energy supply, analyzing policy responses to mitigate global climate change, evaluating environmental cleanup policy, and understanding the consequences of transferring better energy and environmental technology to the former Soviet Union and the developing world.

- Subsurface Characterization. Over the past several years, LBL, with support from the Subsurface Science Program, has developed a unique wide-band, cross-hole seismic imaging system for use in subsurface environments. This system is being used to extrapolate hydrogeologic and geologic information between boreholes in support of field experiments in bacterial transport and microbial heterogeneity, so that disturbance of natural systems in weakly consolidated sediments can be minimized. This technology is now being transferred to the environmental/geologic services and petroleum industries for commercial applications that include subsurface imaging of landfills, abandoned gas stations, commercial waste sites, and heterogeneous petroleum resources, and for mapping the path of steam in secondary oil recovery operations. If successful, single-well seismic imaging would cut the cost of high-resolution subsurface imaging by an order of magnitude while increasing the radius of investigation for submeter resolution by two orders of magnitude over conventional well-logging.

\section{LABORATORY CONSTRAINTS}

Scientific and technological advances are critical if we are to achieve the nation's energy, economic, and environmental objectives. Despite the key role laboratories play in long-term economic security, national laboratory initiatives are constrained by limited budget resources. Constrained funding is a recognized factor in energy research strategic planning. The costs of state-of-the-art facilities and added operational requirements limit the opportunities for setting new research objectives. Under these conditions, it is essential that initiatives be well coordinated and managed and involve effective scientific review. Setting priorities and improving cost effectiveness will be essential to initiating new programs that are vital to the national interest.

The national laboratory system must address performance improvements in many sectors of its research and support operations to meet stakeholder expectations and best management practices. These activities call for improvement in the quality of services and delivery of information at all levels, including environmental, health, and safety programs and financial, 
acquisition, and personnel management systems. A laboratory must have sufficient research funding to support these raised performance expectations. Additional program growth will permit enhancement of LBL's support services while maintaining its high level of research capability and performance.

From the perspective of U.S. industry, national laboratories have sometimes been perceived as enclaves for government-directed research. Contributing to this attitude has been the lack of a specific mission to work with industry and develop the financial resources for successful long-term research that supports critical industrial technology needs. Efforts such as process improvement teams are to remove the barriers to industry-laboratory cooperation and to devote an increasing share of LBL's programs to these activities.

\section{OBJECTIVES AND STRATEGIES}

LBL has a distinguished history of scientific discovery, grounded in a spirit of multidisciplinary teamwork. Many available measures, such as citation analyses, peer reviews, and DOE evaluations, corroborate the Laboratory's preeminent scientific status. However, in spite of LBL's measurable scientific success, LBL supports a diminished share of the total effort of the national laboratory system. This decline does not reflect any change in the ability to deliver on our commitment to research excellence; rather, it suggests a greater need for initiating the new programs demanded by a climate of changing scientific expectations.

An essential part of the new landscape in which these changes must occur is the DOE's emerging strategic outlook-an outlook that recognizes science and technology as one of the Department's "core businesses." Among the goals of this business are the construction of productive user facilities, the application of new technologies to add value to the U.S. economy, and the enhancement of science training and scientific literacy in the U.S. Also included are central research goals, especially important as we consider our own role in contributing to the national interest.

An important DOE research goal focuses on providing "the science and technology core competencies that enable DOE's other business units [energy resources, national security, environmental quality, and industrial competitiveness] to succeed in their missions." In carrying out this enabling research, both basic and applied, LBL has developed internationally recognized programs and facilities. In considering the full range of research that supports the energy, environmental, and competitiveness missions of the $D O E, L B L$ is poised to make significantly increased contributions to the nation. We possess an outstanding set of core competencies, and we are dedicated to conducting research in these areas safely and with full regard for the public's expectations for environmental protection. We are committed to adding value to society by weaving our core competencies into a coherent program for the national laboratory system that carries us from the most basic studies to technological developments of economic value. It is in this context that LBL's agenda for the future has taken shape.

\section{Maintaining Scientific Excellence}

The first part of Vision 2000, to "distinguish ourselves as a premier DOE multiprogram national laboratory by performing research of the highest 
scientific quality," enunciates the single objective that breathes life into all the rest. Without scientific achievement as their ultimate product, partnerships, administrative efficiency, and an empowered work force are empty accomplishments. Our first concern must therefore be a scientific agenda for the next ten years that reflects both the research needs of the nation and the Laboratory's ability to contribute to those needs. The most promising research directions are described below. Among them are endeavors that focus on the energy, environment, and competitiveness missions of the $\mathrm{DOE}$, as well as research directed at a more fundamental understanding of matter and its interactions.

- The Advanced Light Source (ALS) is a crucial part of LBL's future. As a tool for the characterization of advanced materials, the development of $x$-ray lithographic techniques, the study of chemical dynamics, and the pursuit of structural biology research, the ALS has a central role to play in some of the Laboratory's most important research thrusts, at the same time providing great opportunities for researchers across the country.

- The Human Genome Project, in which LBL is a major player, and burgeoning activity in structural biology research serve as both evidence of biotechnology's current vitality and sure signs of dramatic advances in the future. Prospects include a much deeper understanding of the origins of genetic disease, insights into viral structure for custom-tailored pharmaceuticals, and microbial engineering to eliminate contaminants from the soil.

- In high-energy and nuclear physics, astronomy, and astrophysics, LBL has been at the forefront for more than a half century. The quest to understand the universe and the properties of matter underlies fundamental science. Practical applications, such as improvements in medical imaging, also emerge from the scientific achievements themselves and from the technological developments necessary to carry out these challenging research programs. In addition, the intellectual excitement plays a critical role in attracting talented students to the pursuit of science.

- Accelerator and detector technologies sustain central roles in the scientific future of the nation and the national laboratory system. The $\mathrm{ALS}$, and forefront undulator magnet technology, are products of a mature research field that must continue to flourish at LBL. Of particular immediate interest, the Heavy-lon Fusion Accelerator Research effort holds the potential for induction linac technology as a practical means of producing fusion energy. Likewise, the detector technology now finds applications in many fields of science, including medicine.

- LBL must remain at the forefront in providing the nation with a range of technological options to meet both a growing need for energy and an increasing demand for minimizing the environmental impacts of its use. Areas of potential growth include many where LBL has made pioneering advances: development of electromagnetic and seismic methods for imaging subsurface resources, development of advanced building technologies, studies of indoor air quality, electrochemical research and advanced battery development, and combustion research. A particularly important thrust for the Laboratory is research in heavy-ion fusion, where LBL continues to play a pioneering role. 
- LBL is well-positioned for leadership in environmental remediation. Programs include research on improved characterization methods, predictive models and risk-assessment methodologies, techniques to isolate and clean up sites contaminated by radioactive materials and toxic chemical wastes (including bioremediation techniques), and $R \& D$ on waste minimization and effective means of waste disposal, as well as programs of related interest, such as research on indoor air quality. Environmental quality is a national priority, and research aimed at restoring and maintaining the environment is both an opportunity and an obligation of the national laboratory system.

The two steps below are essential elements of LBL's strategy to develop and nurture these new scientific and technological programs and maintain the excellence of research programs:

- Empower leadership for national initiatives. Major initiatives require that resources be mobilized from throughout the Laboratory. In program areas supported as single investigators by DOE, special emphasis must be given to support leadership in emerging areas of science. In addition, LBL must be optimally organized for good pointto-point contact with DOE program managers to advance initiatives of national importance. LBL is working with the DOE to anticipate needs and develop proposals that match the nation's most pressing technological needs. LBL is also working to fully empower initiative champions and have the division directors involved in cross-divisional initiatives.

- Mobilize available fiscal resources for new scientific efforts. The Laboratory-Directed Research and Development (LDRD) funds are vital for catalyzing and fostering new scientific directions and initiatives. LBL is reviewing increased levels of funding for LDRD and assuring the process supports the most promising research directions. The amounts allocated must be large enough to make a difference, to significantly enhance the potential for establishing new directions. At the same time, significant LDRD funds should be reserved for high-risk research whose probability of immediate success is low, but whose long-term scientific payoff may be high.

\section{Adding Value through Partnerships}

The system of national laboratories provides the unique strength of multidisciplinary collaborations for tackling some of nature's and society's most intractable puzzles. Cooperative intradisciplinary efforts among the organizational units within our Laboratory thus remain our most important alliances. We must extend the concept of teamwork even further, from an intellectual network that has linked disciplines within the national laboratories to a productive partnership among the labs, universities, and U.S. industry. A central objective for the next decade must be to enhance our relationships with both American industry and the university community, with the underlying aims being those enunciated in Vision 2000: "to create value for the economy, enhance education, and contribute to the community." Three strategies address strengthening LBL's partnerships:

- Encourage productive partnerships with industry. A powerful force driving the changes in the national laboratories is the environment of global competition. Answering this call entails a true partnership 
between the national laboratories and American industry. Some of the impediments include delays in negotiating industry-laboratory contractual agreements, administrative red tape, and lack of co-funding. Clearly, a more fruitful relationship between the Laboratory and private industry will require not only administrative reform, but also a culture change that must be addressed by the technology transfer process improvement teams.

- Develop partnerships with other laboratories, universities, and agencies. In order to benefit fully from the competencies of the national laboratories, we must partner with federal and state agencies and universities to tackle problems of increasing complexity. We see ourselves as partners in multiagency team projects or serving a wider range of industrial, environmental, and health needs. Partnerships not only among different institutions, but also among different disciplines, are likely to be increasingly productive arrangements at national user facilities such as the ALS. The Human Genome Project is an archetypal example of such a multiagency effort. These partnerships are essential to answer the nation's needs in such areas as materials research and development, environmental research, and biotechnology.

- Strengthen partnerships with the University of California. One of the great strengths of the Laboratory is its close relationship with the UC Berkeley campus. That relationship must take the form of successful joint efforts to pursue major new initiatives. Partnerships must fully join the multidisciplinary academic strengths on campus with the research facilities and technical infrastructure of the Laboratory. Because of the growing importance of interdisciplinary and facilityoriented research (such as the ALS and the Human Genome Project), partnerships with the campus should be strengthened and broadened to address industrial and national $R \& D$ needs and promote leadership in science, mathematics, and engineering.

\section{Optimizing the Way We Work}

In an increasingly competitive national research environment, we need to demonstrate to the DOE and other sponsors that we will spend their research dollars wisely and effectively. The multiplicity of programs at today's Laboratory is both its greatest strength and a potential weakness to developing shared unity of purpose. To strengthen our sense of community, all Laboratory staff must recognize that diverse interests and aims, and a keen respect for individual effort, enhance rather than weaken our ability to reach our goals. A second hurdle is an increasing administrative demand for documentation, paperwork, and adherence to procedural regulation. A mutually respectful relationship and an effective integration of effort are challenges if we are to deliver on our commitment to scientific and technological excellence. Five strategies address these challenges:

- Promote communications among organizations and groups. Good communication plays a key role in working with DOE, other agencies, industry, and key audiences. It can also improve quality and productivity and motivate the Laboratory work force by demonstrating management's respect for every individual. Better communication addresses "us versus them" attitudes that can lead to a balkanization of research and support groups. Among the specific solutions are a 
continuing tradition of working with our customers, town meetings, enhanced systems for desktop communications, a freer and less hierarchical flow of information, a campaign of interoffice and interdivisional information exchanges, and a variety of written and electronic information directories.

- Review policies with a goal of streamlining the bureaucracy. As already noted, a consistent concern at all levels of the Laboratory centers on the increasing burden of regulations, often well-justified but sometimes restrictive and duplicative, and the growing demands for documentation and review. The Laboratory is establishing process improvement teams and working in concert with DOE teams and working groups to address the need for streamlined, efficient, and costeffective management systems.

- Promote a cooperative spirit among service providers and "customers." Scientists quite properly look increasingly to user facilities that facilitate rather than hinder their research and that minimize cost and inconvenience. Satisfied customers should be recognized as the main deliverable of our user facilities. In management support areas a strong customer orientation is required. This strategy includes efforts to assure adequate technical resources and skilled support staff, resource analysis to ensure that facilities are fully utilized but not overcommitted, expedited handling of facility visitors ("one-stop shopping"), and a host of user-friendly amenities. More broadly, a commitment to cooperation in pursuing research excellence should be the central focus of administrative and support elements at the Laboratory.

- Promote on-line systems for administrative functions. An essential tactic in implementing each of the three strategies just discussed is a greater reliance on electronic communications and electronic information management. Despite the Lab-wide use of faxes, voicemail, electronic mail, and electronic information transfers, the penetration and use of these newer communications technologies are uneven throughout the Laboratory. Such services should be available at each employee's work area. An attainable objective must be to replace virtually all of the Lab's paper transactions with on-line systems that make forms and procedures current and accessible, and that allow users to check the status of pending actions.

- Advance DOE's Total Quality Management Initiative. Many of LBL's strategies and initiatives previously taken in connection with the Tiger Team corrective action plan, the Self-Assessment Program, and the new maintenance and operations $(M \& O)$ contract between the University of California and the DOE, can be seen as elements of "total quality management" at LBL. However, to complete this quality program at the Laboratory-a process that we see as an evolutionary one-we must make two additional Lab-wide changes. First, we must promote a culture where we measure and analyze our results from a quality perspective. The second change is the emergence of a culture in which all employees participate in the development of the Laboratory. Management must seek input, advice, and suggestions from employees on all the work processes that affect them. And employees must be visibly recognized and rewarded for their contributions to the institution. $L B L$ is implementing training for employees to address this strategy. 


\section{Respecting and Ennpowering Our People}

This management objective is to develop our people to their fullest potential, value and seek diversity in our work force, and create an environment that respects the individual and fosters integrity. The objective is fundamental - to ensure a unity of purpose, a respectful sense of community without which our research objectives surely become unattainable. To broaden the dialogue between senior management and Laboratory staff, specific training and task forces continue their "focus group" meetings, moderated by the Deputy Director. Several strategies have been defined:

- Enhance communication as a means of unifying our community. A number of specific actions are being implemented to address this strategy, including town meetings, enhanced electronic communications, and more interdivisional meetings. To break down hierarchical rigidity in the dispersal of information, it is equally necessary to encourage rapid vertical diffusion of accurate information and feedback. Successful communication will mean conveying more directly, even personally, a message of respect and appreciation, not just current information, from upper management to all levels of the organization.

- Establish and sustain a diverse work force at the Laboratory. Although Laboratory programs related to diversity in the work force have been in place since the mid ' 80 s, the term work force diversity has recently come to have a much broader meaning than mere gender and racial balance. The challenge and the goal is to value diversity in the workplace, and to establish diversity as a permanent part of the Laboratory's institutional culture. We are committed to equal opportunity and affirmative action, and we recognize these policies as the first and most important steps to achieving diversity in our working community. However, unlike equal opportunity and affirmative action, achieving work force diversity is not simply a matter of compliance with the law; rather, it is a process that aims to build a community in which fairness and equity exist across the board for all individuals. The section on Critical Success Factors in this plan delineates steps undertaken to address LBL workforce diversity needs.

- Train managers in leadership. LBL managers are often chosen primarily for their scientific achievements and scientific imagination. However, their abilities to organize and manage or to inspire and lead a diverse staff are now critically important. An effective scientific leader cannot ignore the importance of a range of management skills, especially with regard to communications, interpersonal relations, and personnel development. Management should provide "goals and guidelines," then give "scientific, technical, and administrative personnel the freedom and support necessary to develop their own solutions." Our personnel must be empowered to solve problems rather than simply carry out solutions invented by others. Skill in moving toward the goals of staff development, open communications, and employee empowerment is a key aim of LBL's management training.

- Encourage career growth for all our people. More educational opportunities and job-related training and a wider awareness of opportunities are measures for addressing the professional development of LBL employees. Innovative approaches are also being addressed and considered, including mentoring programs, job rotation, 
and short-term interdivisional job assignments, among others. The goal in all cases must be a realization on the part of all of our people that their development and growth as LBL employees should be limited only by their own ambitions and aptitudes, and facilitated by a responsive management.

- Broaden the practice of mentoring. Mentoring, cultivating a relationship in which an experienced employee gives personal and professional guidance to an employee with less experience, encourages employee empowerment, career growth, and better communication across the Laboratory. A mentor guides a protégé by taking a more active role in his or her career development and provides an anchor to the organizational culture. While informal mentoring has taken place at LBL since the early days of the Laboratory, not all new employees establish such relationships. By formalizing mentoring, we can not only sanction the mentoring relationship, but also ensure that the benefits of mentoring are available.

\section{STRATEGIC ACTIONS}

LBL is undertaking a range of scientific, operational, and administrative steps to implement the strategic plan and address the Laboratory's needs for the next decade. The following seven steps mobilize LBL strengths through investing in the future: in our science, in our infrastructure, and in our people. These actions are only the first steps, albeit important and thematic ones, of a plan that will be further defined and elaborated in the coming months and years.

- Allocate enhanced resources to support high-priority initiatives. These resources will come from an increase in and some redirection of Laboratory resources. For the Laboratory Directed Research and Development funds a target for the ultimate size of the LDRD lies between $4 \%$ and $6 \%$ of the Laboratory operating budget, which will better support critical new directions without diminishing our ability to encourage high-risk research. In addition, major Laboratory initiatives will have dedicated leadership to address major national science and technology issues.

- Establish a strong industrial program. Parallel with our strengthened commitment to new initiatives, the Laboratory must heed the growing expectation that scientific research deliver measurable value to society. A strong industrial program, supported in part by resources from the new technology transfer programs of the DOE, will ensure that our strengths in such fields as structural biology, materials science, and energy efficiency technologies are productively linked to the commercial sectors that can exploit them. Our goal is a level of precompetitive research that will bring in industrial funding equal to a significant fraction of our annual operating budget.

- Reinforce our historic partnership with the University of California at Berkeley. We will move vigorously to fortify the Laboratory's historic partnership with the Berkeley campus by engaging in joint planning at all levels. Already under way is a joint planning activity involving managers at the highest levels of the University and the Laboratory. Ultimately, upon realizing the goals embodied in this and the previous 
action, we envision a three-way cooperative link among industry, academia, and the Laboratory-a broad partnership that will put us in a much stronger position to exploit the opportunities of a changing scientific landscape.

- Implement actions for making an LBL that works. Laboratory management has endorsed specific actions for administration, technical support infrastructure, and information and computing. In all cases, the goals of the recommendations are greater efficiency and more productive, mutually respectful relationships. These include developing a more seamless information and computing environment, reductions in paperwork, elimination of redundant activities, and streamlining decision making.

- Provide training to enhance skills, career opportunities, and employee development. Management and leadership training for our employees reflects an evolving consensus that management today is about empowerment-that team-building and communications are more essential skills than ever before. Further, we are putting increasing emphasis on formal career planning for each career employeeplanning that includes training appropriate to each employee's skills and performance, and aligned with realistic career goals within the Laboratory's job classification structure. The central aim will be to reinforce strengths and correct weaknesses as a means to realizing each employee's full potential. A new program of supervisory training emphasizes the building of general supervisory skills, as well as the basics of leadership behavior. Among its tenets are the principles of maintaining the self-esteem of others, developing and maintaining productive relationships, and leading by example.

- Establish a broad communications program. A Communications Task Force has been established to address all aspects of LBL communications. Key external communications issues include conveying an accurate picture of the Laboratory to the scientific community, to our sponsors, and to the public, both locally and nationally. Internally, initial actions include training people in communications (as part of the training effort above); enhancing "real-time" intralaboratory communications by means of electronic bulletin boards, providing a means for all employees to make their views known on Laboratory issues and problems; and increasing the availability and visibility of Lab management. This last commitment consists of all-employee divisional meetings and town meetings to discuss matters of broad concern within the Laboratory.

\section{MANAGEMENT ISSUES}

To maintain LBL's scientific leadership and to ensure the full development of its capabilities and resources, LBL has identified strategic management issues that it is addressing with DOE through institutional planning and other management forums. These issues, which continue to be developed as an ongoing process within LBL's strategic planning activities, can be divided into five main categories: fulfilling our mission, implementing initiatives, modernizing our research facilities, further improving our ES\&H performance, and improving efficiency in oversight and indirect costs. 


\section{Fulfilling Our Mission}

The following are some key activities LBL needs to address as part of the implementation of its strategic plan.

- Enable industrial R\&D partnerships. The Laboratory is working with DOE to develop work-for-others conditions and Cooperative Research and Development Agreement documents and procedures that reduce obstacles to the development of R\&D partnerships and technology transfer.

- Establish further alliances and agreements with NIH, DOD, NASA, EPA, and other agencies. The Laboratory's research relationships with the National Institutes of Health (NIH), Department of Defense (DOD), National Aeronautics and Space Administration (NASA), and U.S. Environmental Protection Agency (EPA) have strengthened national research programs in space research, health, and environment. Agreements need to be forged to reinforce these mutually constructive relationships and reduce administrative barriers.

- Fully utilize Laboratory and university capabilities. LBL is unique among the multiprogram national laboratories in being located next to a large university. The Laboratory and DOE must continue working constructively to strengthen relationships with the University of California Berkeley campus as well as with other universities, and to devise an administrative framework that minimizes barriers to university and other partners seeking access to facilities.

\section{Implementing Initiatives in Core Business Areas}

Limited DOE resources set constraints on the range and scope of LBL's initiatives. In this environment, scientific excellence alone is no guarantee of success; the Laboratory must also demonstrate that its initiatives contribute to national needs. The Laboratory is working with DOE, the scientific community, the state, and industry participants to establish priorities and to support initiatives that can best serve the nation under financial constraints. Examples from four key DOE program areas illustrate the Laboratory's priorities for initiatives.

- Office of Basic Energy Sciences. The Laboratory proposes additional beamlines and the completion of the second floor of the Advanced Light Source building for user offices and laboratories. The Combustion Dynamics Initiative, proposed in partnership with Sandia National Laboratories, supports the DOE's Chemical Sciences Division commitment to the nation's environmental, energy supply, and fundamental science goals. The Laboratory is working with broader state and national scientific communities to seek additional support for this project to complement DOE funding.

- Office of Health and Environmental Research. LBL's Human Genome Center and the Advanced Light Source give biologists access to advanced technology needed to address fundamental life science challenges: to understand the genetic and structural basis of energyderived and environmentally derived health issues. LBL is working with the Office of Health and Environmental Research to establish a scientific and management framework that will ensure the success of these initiatives. 
- Office of Fusion Energy. The proposed Elise accelerator project is poised to evaluate the potential for using intense and focused heavyion beams as drivers for inertial confinement fusion. The Laboratory is prepared to construct this test facility, as well as to continue research in other national fusion programs.

- Assistant Secretary for Energy Efficiency and Renewable Energy. LBL proposes to assist DOE in its efforts to implement actions to reduce greenhouse gas emissions (the Climate Change Action Plan, or CCAP). This initiative consists of three distinct elements, each of which is important to the nation if the CCAP is to be successful: (1) assistance with specific elements in the CCAP (e.g., appliance standards); (2) monitoring and evaluation (e.g., of buildings retrofitted to be more energy efficient); and (3) developing a research base of advanced energy efficiency technologies and methodologies (e.g., advanced techniques for building design).

\section{Modernizing Facilities}

The Laboratory will continue to give priority to modernizing and restoring facilities to sustain national programs while also achieving standards of excellence in the areas of environment, health, and safety. The Laboratory has integrated its work on the Site Development Plan with the processes of institutional planning, safety and health planning, and environmental restoration so that a safe working environment will be provided for implementation of DOE scientific programs. Critical elements of LBL capital resource and modernization planning are implemented through:

- Multiprogram Energy Laboratories Facilities Support (MEL-FS). This program has been vital for replacing mechanical and electrical utilities, upgrading buildings, and improving segments of roadways at LBL. A key element for the program in the 1990 s will be the continued improvement of these systems and modernization of support buildings and general purpose research facilities. Recent reversals in the program's funding place the Laboratory infrastructure under severe stress.

- General Plant Projects (GPP). Small capital projects $(\$ 100,000$ to $\$ 2.0$ million) supported by GPP are essential for small program modifications and additions, for compliance with environmental, health, and safety standards, and for upgrades of obsolete and deteriorated equipment such as transformers, switching stations, boilers, chillers, and roofs. At $\$ 3.5$ million, the current annual budget for GPP projects is inadequate to make substantial progress in meeting identified needs. Note that funds are allocated in accordance with the DOE CAMP system, which is integrated with the ES\&H Management Plan.

- General Purpose Equipment (GPE). The Laboratory uses GPE funds to replace its essential support equipment. This equipment includes environmental, safety, and health equipment; mechanical and electrical engineering shop equipment; transportation vehicles, including shuttle vehicles; data processing and telecommunications equipment; and other equipment used by support divisions. In FY 1994, GPE funds are $\$ 1.8$ million, but, compared with 1981, the purchasing power is equivalent to about $\$ 700,000$, or one-half the 1981 funding. This level of need has resulted in equipment used beyond its normal 
lifetime, sustained high maintenance costs, and substandard equipment performance.

- Recycling Bevalac Shielding Blocks. The Bevalac accelerators were shut down in FY 1993. Plans called for Energy Research support for the stand-down and secure phase into FY 1994. The prospect of recycling shielding blocks at Brookhaven's RHIC offers the potential for very significant waste minimization and cost savings for DOE.

\section{Improving ES\&H Performance}

It is the policy of the Laboratory to integrate its performance in the areas of environment, safety, and health (ES\&H) into the planning and conduct of all of its operations to protect the health of employees, the public, and the environment. The Laboratory has developed a comprehensive Corrective Action Plan, an Environment, Safety and Health Management Plan, and a five-year Environmental Restoration and Waste Management Plan. These plans integrate ES\&H requirements into all Lab activities in a prioritized manner, to assure that LBL can meet DOE's Critical Success Factors for ES\&H in the pursuit of world-class research. All new initiatives include specific identification of ES\&H needs, and their Conceptual Design Reports and other supporting materials indicate ES\&H requirements for program planning. Issues that continue to be addressed include:

- Resources for required environment, safety, and health plans. Management arrangements and interactions with DOE are being strengthened, and commitments must be obtained from DOE to ensure that the ES\&H momentum can be maintained. LBL has committed substantial resources to EH\&S functions, but emphasis on documentation and compliance regardless of risk has diverted resources from needed improvement projects as well as having been insufficient to fully develop and implement LBL's ES\&H plans and programs.

- ES\&H facilities for essential programs. As indicated by the Tiger Team, many of LBL's ES\&H facilities are inadequate and in poor condition. Additional facilities are necessary to correct deficiencies identified by the 1991 Tiger Team Assessment, implement the Corrective Action Plan, and fulfill the personnel and program obligations in the Environmental Restoration and Waste Management and the Environment, Safety and Health five-year plans. Construction has begun on a new Hazardous Waste Handling Facility to upgrade, consolidate, and relocate existing waste handling operations. Construction is scheduled for completion in 1996.

\section{Performance-Based Management}

Appendix $\mathrm{F}$ of the contract between the University of California Regents and the Department of Energy for the operation and management of LBL contains performance measures that are the components of the performance-based management system that the University and DOE utilize for Laboratory oversight. The DOE and the University have developed the measures as clear and reasonable objective standards against which the University's overall performance under the prime contract will be assessed. The performance measures are subject to annual review and may be modified by the agreement of the parties. 
In addition, a self-assessment methodology has been established. The guidelines for science and technology assessment are summarized in a document entitled "Laboratory Science and Technology Assessment Process and Criteria." In essence, these guidelines prescribe the use of outside peer review committees, which assess the laboratories' science and technology programs according to four criteria: quality of science, relevance to national needs and agency missions, performance in the construction and operation of major research facilities, and programmatic performance and planning. The result of each review is an overall rating of outstanding, excellent, good, marginal, or unsatisfactory. Furthermore, an extensive self-assessment program for all LBL management, administrative, and operational activities has been established with a particular focus on the environment, safety, and health performance of the Laboratory as reviewed in Section V.

The long-term goal for LBL's performance-based contracting is the integration of the measures, assessments, and reviews for an efficient and productive evaluation of LBL's performance. The Laboratory and the University will continue to work with DOE to optimize the performance evaluation process, eliminate redundancies, and develop a system that provides positive feedback for improved performance.

\section{Improving Oversight Efficiency}

$D O E, L B L$, and $U C$ need to work together to eliminate redundancy in the review process, such as minimizing unnecessary paperwork and lengthy review cycles in the existing procedures for compliance presently required for the National Environmental Protection Act (NEPA). Performance based management and the Self-Assessment Program hold promise for improving the efficiency of Laboratory operations.

- Paperwork reduction. The existing framework for Cooperative Research and Development Agreements must be streamlined and made more timely. In addition, paperwork required for NEPA compliance should be reduced by consolidating similar projects. Both of these actions would facilitate effective decision making and encourage industrial partnerships. The DOE-Laboratory process improvement team is working to address this issue.

- Excessive reviews. LBL program and support activities are subject to more than 300 reviews annually. These costly reviews require considerable time and effort by both the Laboratory and DOE. By emphasizing biennial and triennial reviews, DOE and the Laboratory could work together to streamline the review process, increase productivity, and reduce costs. The current performance-based contract between the University of California Regents and the Department of Energy calls for the elimination of redundant oversight.

DOE, LBL, and UC need to work together to better understand the drivers of indirect costs. Opportunities for consolidating redundant and/or non-value-adding activities should be identified. 


\section{$I V$}

The strategic planning efforts of Lawrence Berkeley Laboratory (LBL) support four business areas of the Department of Energy (DOE):

- Science and Technology

- Energy Resources

- Environmental Quality

- Industrial Competitiveness

The programs supporting these strategic areas, although founded on a tradition of excellence in fundamental and applied research, evolve in response to new challenges and national priorities. The following sections each describe one of these four business areas and discuss the initiatives proposed. The research and development directions in each area are also described.

\section{SCIENCE AND TECHNOLOGY}

LBL's science and technology business area is directed to build and make available the unique resources of the DOE laboratories to maintain leadership in basic research, to focus on fundamental and applied research in support of the DOE and other federal and state agencies, and to maintain world technical leadership through science and mathematics education. The key program areas include energy research and the DOE's other science and science education activities. The following sections identify key LBL initiatives in support of these core areas and describe research and development directions. It is important to recognize the overlap of these initiatives with other core business areas. Examples include the applicability of Atomic-Scale Synthesis of Advanced Materials to Industrial Competitiveness, 
and Combustion Dynamics to Environmental Quality. LBL's science and technology initiatives include the following:

- Advanced Light Source Science and Technology

- ALS Beamlines

- Combustion Dynamics

- Structural Biology

- Genome Sequencing

- Transgenic Initiative

- Atomic-Scale Synthesis of Advanced Materials

- Electron Beam Microcharacterization Facility

- B Factory at Stanford Linear Accelerator

- Large Hadron Collider

- Relativistic Heavy-Ion Collider Program

- IsoSpin Laboratory

- National Information Infrastructure: Networking

\section{Advanced Light Source Science and Technology Initiatives}

The Advanced Light Source (ALS) produces the world's brightest light in

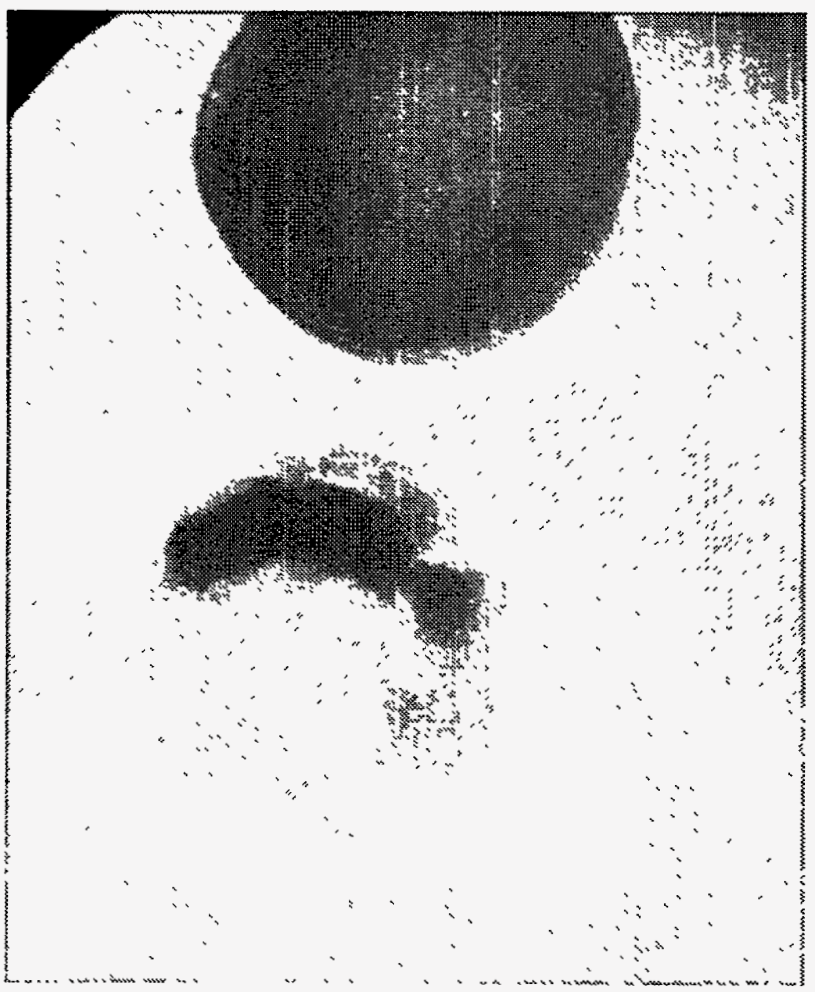
the soft $x$-ray and vacuum ultraviolet range of the spectrum. This synchrotron radiation source is used for basic and industrial research and development across a broad spectrum of the physical, chemical, and life sciences, as well as such technological areas as materials analysis, microstructure fabrication, and macromolecular crystallography. To exploit fully the state-of-the-art capabilities of this newly constructed national user facility, the Laboratory has developed three major initiatives that, collectively, would provide for installation of the full complement of insertion devices (undulators and wigglers) in the ALS storage ring, full instrumentation of the insertion-device beamlines, and completion of the second floor of the ALS building with user offices and laboratories, thereby enhancing the ability of the ALS to expand the nation's scientific and technology base. The ALS Beamlines Initiative will provide specialized facilities for high-spatial-resolution $x$-ray microscopy with emphasis on systems of industrial interest and high-resolution spectroscopy of materials and biological systems at high photon energies with both linearly and circularly polarized x-rays. In collaboration with the Sandia National

The High-Resolution Zone Plate Microscope, on the Advanced Light Source's microscopy beamline, is dedicated to highresolution microscopy for biology and materials science applications. The image shows a malaria-infected human red blood cell at a resolution of less than 0.1 micron; planned improvements should enhance that resolution to approximately 5 times that available using visible-light microscopy. $X$-ray microscopy using synchrotron radiation offers researchers a unique opportunity to image biological systems in near-native states (in water, at atmospheric pressure). 
Why is this solar cell defective? The answer was found by analyzing the chemical composition of the cell in microscopic detail using the $x$-rays of the ALS. A major benefit of the brightness of the ALS beam is high spatial resolution and high spectral resolution, which in this case allowed the detection of trace amounts of iron that were probably introduced during fabrication and. led to the cell's failure. The peaks represent the amount of iron in the defective cell.
Laboratories (SNL), the Combustion Dynamics Initiative will provide the facilities that will enable the ALS to support chemical dynamics research that underlies solutions to key problems in energy development and efficiency. The Structural Biology Initiative will provide the facilities for advanced imaging of biological structures in near-natural environments, diffraction for structural determinations of proteins and other biological macromolecules, and spectroscopy using polarized radiation to investigate metallo-organic molecules.

ALS Beamlines Initiative

To realize the full scientific potential of the ALS for both fundamental and applied research, LBL is proposing an initiative for experimental facilities needed to address new forefront scientific and technological opportunities identified by the nation's research communities. Applications of the ultrahigh-brightness ultraviolet and soft $x$-ray radiation from the ALS cover diverse areas, including atomic and molecular physics; biology and medicine; chemical dynamics; materials, interface, and surface science; and industrial research and technology:

- Several of the most exciting applications of the ALS lie in the field of high-resolution x-ray microscopy, in which enhanced spatial resolution is a significant consequence of the high brightness of the ALS. The impacts of $x$-ray microscopy are expected to be broad because of the decreasing size of the physical and biological systems to be analyzed.

- The pulsed time structure of the ALS is crucial to the area of chemical dynamics, which encompasses all phenomena in which molecules undergo energetic or chemical transformations, both in the gas phase and on solid surfaces.

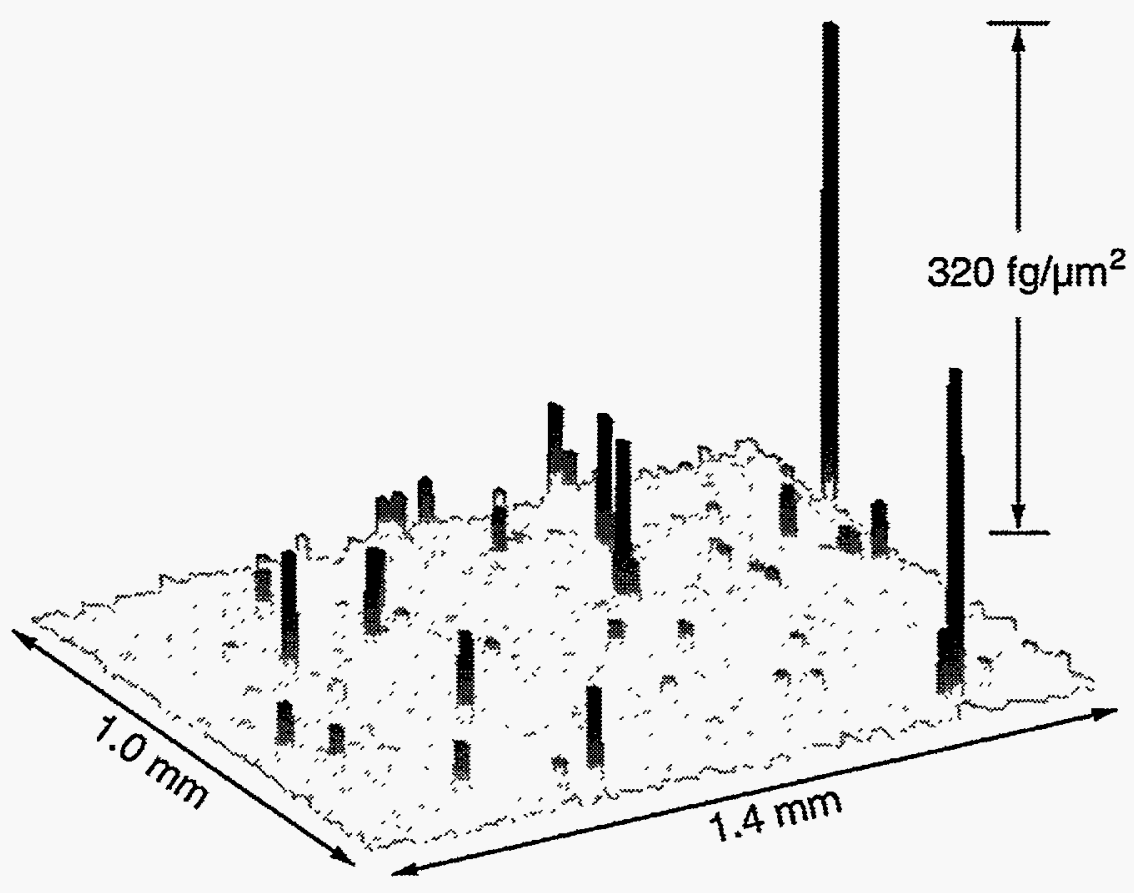


- The availability of broad-spectrum x-ray radiation is essential for detailed spectroscopic studies of solid-state materials and gas-phase atomic and molecular systems.

- In all fields, the extra degree of freedom associated with circular polarization lends a precision and specificity to photon-based techniques that would otherwise be lacking. The use of circularly polarized synchrotron radiation is now at the cutting edge of research on properties, such as magnetism, that are dependent on electron spin.

Full utilization of the ALS to exploit emerging needs of users from industry, academia, and government laboratories requires insertion devices (undulators and wigglers) and beamlines to carry the light from insertion devices and bend magnets to the experimental end stations, together with completion of the $1877 \mathrm{gsm}(20,200 \mathrm{gsf})$ of office, light laboratory, and support space for users in the unfinished second floor of the ALS building. Project costs include all safety systems necessary for full compliance with applicable regulations, orders, and ALS design specifications. In compliance with the National Environmental Policy Act (NEPA), an Environmental Assessment was prepared for the ALS and a finding of No Significant Impact was issued in 1988. In compliance with the California Environmental Quality Act (CEQA), an Initial Study report was prepared and a Negative Declaration adopted in 1987.

ALS Beamlines Initiative Resource Requirements $(\$ M)^{\mathrm{a}}$

\begin{tabular}{lcccccccc}
\hline \multirow{3}{*}{ Category } & $\begin{array}{c}\text { Prior } \\
\text { Years }^{\mathrm{b}}\end{array}$ & 1995 & 1996 & 1997 & 1998 & 1999 & 1999 & Total \\
\hline Operating & 0.1 & 0.0 & 0.0 & 3.0 & 2.0 & 3.0 & 0.0 & 8.1 \\
Construction & 0.0 & 0.0 & 0.0 & 9.0 & 20.9 & 22.7 & 0.0 & 52.6 \\
\hline
\end{tabular}

aPreliminary estimate of actual-year LBL Budget Authority (B\&R code KC), per 1994 Conceptual Design Report.

b For production of conceptual design report and environmental assessment.

\section{Combustion Dynamics Initiative}

In support of DOE's national role in combustion research and chemical science, LBL and SNL have proposed a Combustion Dynamics Initiative. This initiative advances DOE's energy sciences mission by providing the knowledge and technology base needed to help solve the nation's combustion-related problems of the 21 st century. DOE's Basic Energy Sciences Advisory Committee (BESAC) reviewed the initiative in depth in 1992 and again in the Spring of 1994, and gave its strong endorsement to the effort at Sandia and Berkeley. The Chemical Dynamics Research Laboratory (CDRL), LBL's component of this initiative, will be a dedicated facility open to all qualified investigators. The CDRL will exploit recent advances in chemical dynamics, in conjunction with the ALS, which produces the brightest ultraviolet light available anywhere in the world, to expand the understanding of fundamental chemistry relevant to combustion. The CDRL will also contribute to the knowledge base on which rests the conservation energy programs in DOE, including those supporting efficient use of methane and related fuels. 
The Advanced Light Source Science and Technology Initiatives are directed toward full utilization of the state-of-the-art capabilities of the ALS. When these initiatives are realized, this national user facility will have a full complement of insertion devices in the storage ring, full instrumentation of the insertiondevice beamlines, and completion of laboratory and office space.

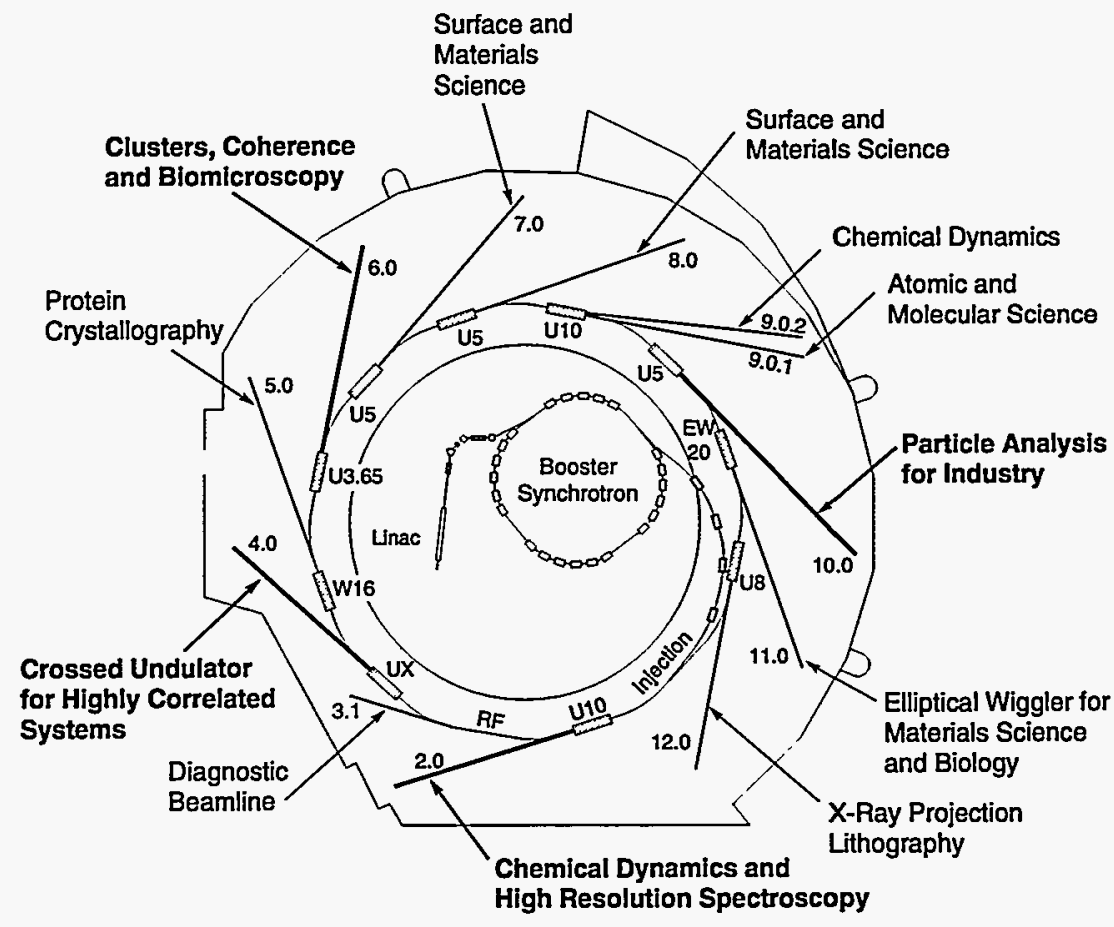

The CDRL will provide experimental resources for national users to enable new investigations of fundamental and applied combustion processes. At LBL, new lasers, experimental stations, and dedicated chemical physics beamlines from the ALS will be developed for dynamic, spectroscopic, and structural studies of many types of highly reactive molecules, radicals, clusters, and unusual transient species. A rigorous molecular-level understanding of combustion reactions, the structure and dynamics of highly excited molecular species and reactive intermediates, and molecular energy flow processes can provide basic new knowledge that underlies scientific and technological leadership in internationally competitive energy-related and alternative fuels industries. Application of this basic chemical knowledge will be accelerated by the partnership with SNL through complementary experimental resources for applied research and linkages to combustion researchers in industry and universities.

Research results from the CDRL will be incorporated into powerful predictive computer codes under development in the Combustion Dynamics Initiative. These computer codes will enable a broad range of manufacturers to design clean, efficient combustion systems.

The CDRL will host visiting scientists, and its facilities will be made available to collaborating investigators. A Program Review Panel, reporting to the highest levels of management, will recommend allocations of resources and review all proposals for use of the experimental stations. LBL's outstanding graduate student and science education programs will contribute to full utilization of the facility in support of national science education goals. The planning of this initiative has included rigorous safety, health, and environmental considerations to ensure that the project will be conducted in full compliance with DOE Orders, NEPA and CEQA, and other federal, state, and local regulations. It is anticipated that an Environmental Assessment will be prepared under NEPA and an Initial Study will be prepared under CEQA. 
Combustion Dynamics Initiative Resource Requirements $(\$ M)^{\mathrm{a}}$

\begin{tabular}{lccrrrrl}
\hline Category & 1995 & 1996 & 1997 & 1998 & 1999 & 2000 & Total \\
\hline Operating & 0.0 & 1.0 & 1.3 & 2.4 & 2.5 & 2.8 & $10.4^{\mathrm{b}}$ \\
Construction & 0.0 & 6.6 & 14.5 & 16.7 & 15.4 & 8.8 & 61.9 \\
\hline
\end{tabular}

aEstimates of actual-year Budget Authority for construction and related project costs for the Chemical Dynamics Research Laboratory (B\&R code KC).

bDoes not include \$0.3M R\&D conducted in FY 1992.

\section{Structural Biology Initiative}

New applications of advanced imaging, diffraction, and spectroscopy techniques will greatly strengthen DOE's emerging national program in structural biology. A structural biology research program is being formulated at LBL to develop the potential structural biology resources at the Advanced Light Source. The ALS will offer major new resource opportunities for life sciences research in several emerging areas of scientific emphasis:

- X-ray crystallography-to conduct static and dynamic analysis of macromolecular architecture with precise wavelength tuning. The facility addresses the high demand for beam time with a multiuser configuration and user-friendly design and operation.

- X-ray spectroscopy-to determine biochemical properties at high spatial and temporal resolution within cells and organelles. Many experiments will exploit the capacity to control the polarization of synchrotron radiation.

- X-ray microscopy-to investigate tissues, cells, and organelles in nearnative states at a resolution that may approach macromolecular dimensions. Among the benefits of $x$-ray microscopy at the ALS will be element-specific imaging and the ultimate possibility of threedimensional imaging.

The structural biology programs at the ALS will rely initially on experimental stations at the ends of three beamlines. The first, from a wiggler

A layout is shown of the protein crystallography beamline that is part of LBL's Structural Biology Initiative. This new capability will support research for a wide variety of applications, including the determination of how proteins regulate the expression of genes by examining the structure of the protein-nucleic acid complexes, and the study of protein microcrystals.

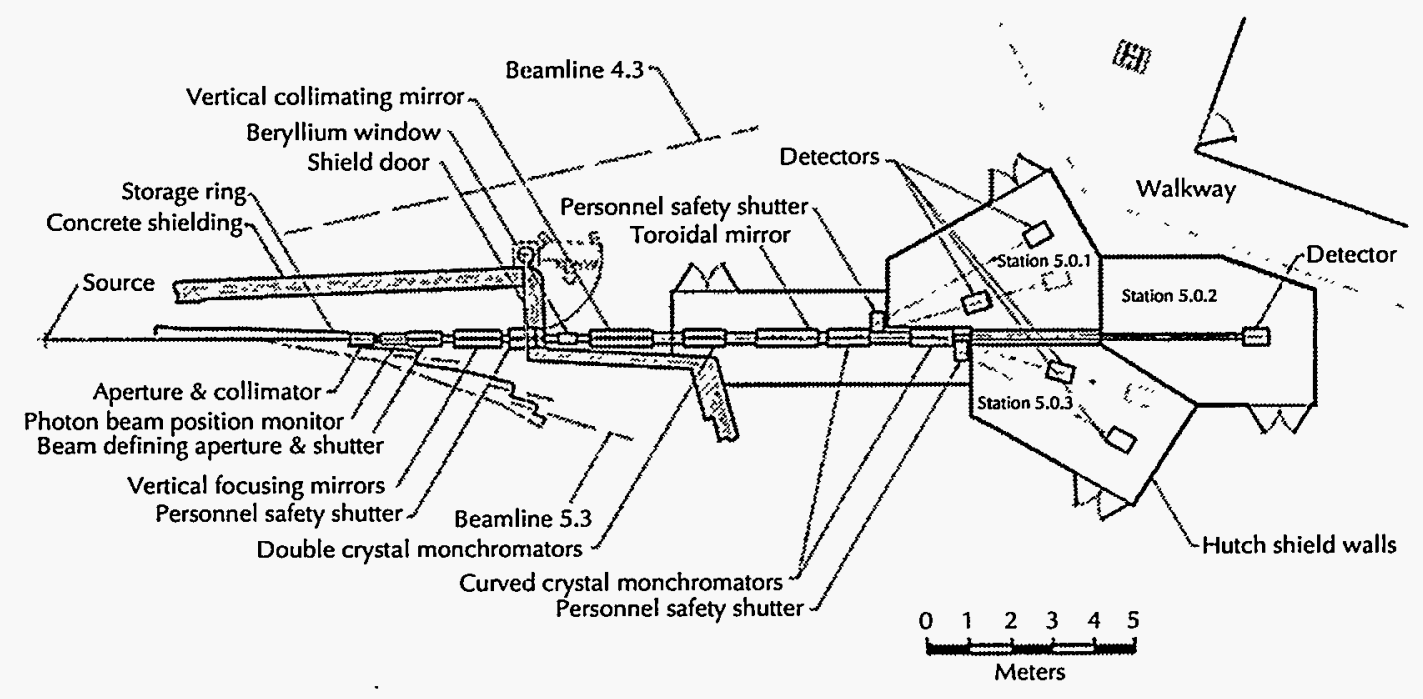


source of both soft and hard $x$-rays, will branch into several experimental areas for both $\mathrm{x}$-ray crystallography and spectroscopy. The second beamline will come from a polarized wiggler for applications in structural biology and the materials sciences. The third beamline, from an undulator source of ultrabright soft $x$-rays, will illuminate two $x$-ray microscope stations, one available for biological microscopy and the other for developing advanced microscopy techniques. The majority of the operating (and equipment) funds identified below will be for fabrication and development of these beamlines. The supporting laboratories will be constructed on the second floor of Building 6 and in the adjacent Building 80, as part of the ALS Structural Biology Support Facilities. A categorical exclusion under NEPA and a categorical exemption under CEQA were prepared for associated modifications to Building 80 .

These ALS projects will be coupled to other structural biology expertise at LBL to initiate new collaborations and to participate in the national scientific program. Other relevant strengths at LBL include electron crystallography, high-voltage electron microscopy, nuclear magnetic resonance (NMR) spectroscopy, time-resolved Fourier-transform infrared spectroscopy, and scanning tunneling microscopy.

Structural Biology Initiative Resource Requirements $(\$ M)^{\mathrm{a}}$

\begin{tabular}{lccccccc}
\hline Category & 1995 & 1996 & 1997 & 1998 & 1999 & 2000 & Total \\
\hline Operating & 7.5 & 8.2 & 5.5 & 4.1 & 4.1 & 4.1 & 33.5 \\
Construction & 4.7 & 2.6 & 0.0 & 0.0 & 0.0 & 0.0 & $7.9^{\mathrm{b}}$ \\
\hline
\end{tabular}

apreliminary estimate of actual-year LBL Budget Authority (B\&R code KP).

bIncludes prior year funding.

\section{Genome Sequencing Initiative}

A Genome Sequencing Initiative is being developed to leverage the technology and expertise that thrive in the Lawrence Berkeley Laboratory's Human Genome Center (HCC) toward megabase sequencing challenges. The HGC is oriented almost exclusively towards developing and implementing directed methodologies for cost-effective and accurate high-throughput human DNA sequencing. This work has five components. The first three components of the Center are all involved with new technology development for sequencing and are based on a collaboration between biologists, the automation group, and computer scientists. The fourth component is the sequencing production effort itself. The fifth component of the HGC is the biology effort that interfaces with and performs experiments derived from the completed sequence data.

The first component of the HGC, the biology component of the new technology group, has developed a directed strategy of DNA sequencing in which high-resolution physical maps are generated so that a small set of standard primer binding sites are positioned every 300 base pairs. This mapped set of templates is then sequenced. Using this strategy, templates can be selected in a minimally redundant fashion, which means that template preparation requirements are reduced tenfold and sequencing reactions can be reduced fivefold. In addition, sequence assembly is straightforward because all the templates are mapped in relation to each other, with a resolution of about 30 base pairs, prior to sequencing. The biology group is continuing to optimize the biological procedures of the directed process. 
In support of DOE's Human Genome Project, $L B L$ researchers have combined their expertise in biology, automation and informatics, to implement a "directed" sequencing strategy that has led to dramatic improvements in sequencing efficiency. The strategy, which allows for a minimal set of $P 1$ sequencing subclones to be selected, reduces needless redundant sequencing. The oligonucleotide sequencer shown here, an LBL innovation that has enabled cost savings in sequencing reagent use, has become the focus of commercial interest by the biotechnology industry.

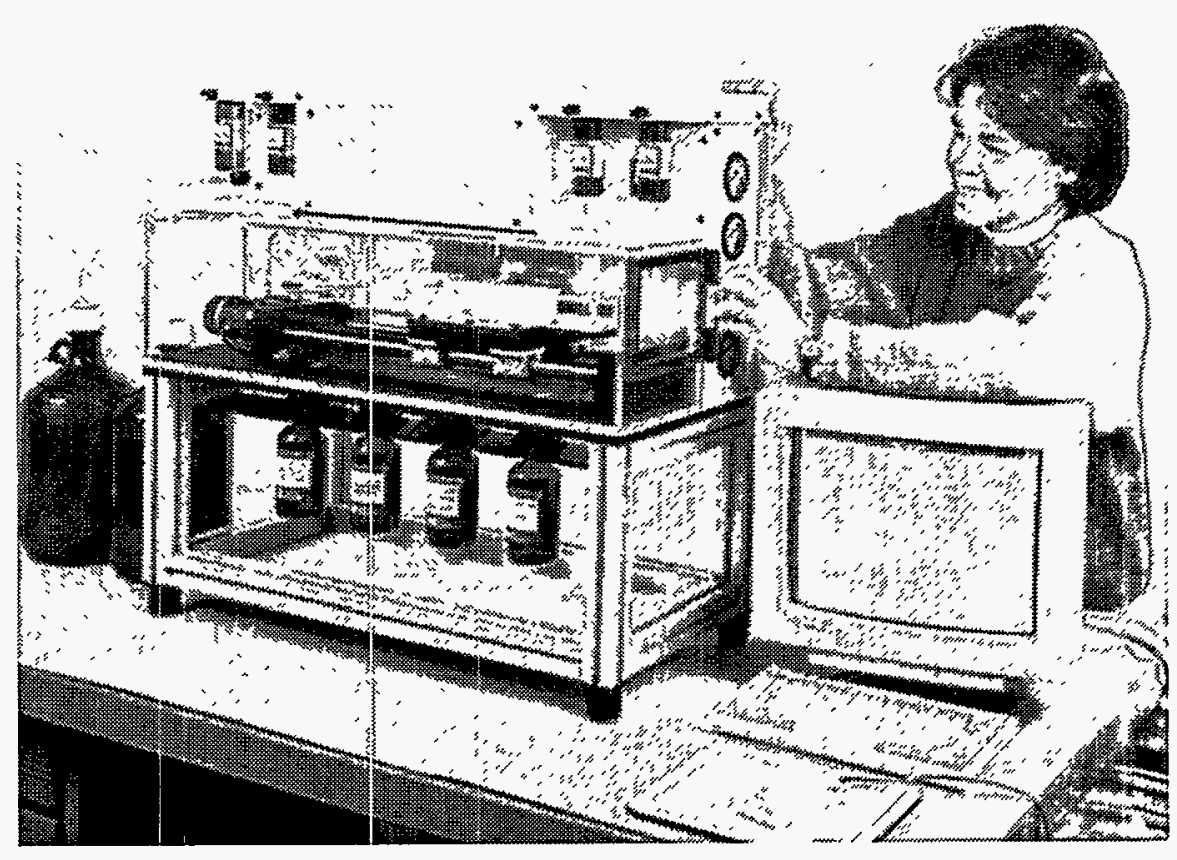

The second component of the Center is the automation group, which is developing instrumentation to support the directed sequencing approach. Some of the modules have been completed and are currently in use: an image station that captures and analyzes the mapping information from agarose gels, a colony picker, a robotic library replicator, and a modified Biomek that sets up polymerase chain reaction assays and sequencing reactions. A water-based thermocycler and a 12-channel oligonucleotide synthesizer are being rigorously tested by the biologists prior to entering production. Novel methods to analyze polymerase chain reaction fragments as well as preliminary plans to integrate some of the initial modules are in the early planning stages.

The third component of the HGC is the informatics group. The major goal of the group is to develop software that facilitates the sequencing effort. The developmental effort is aimed at all aspects of the process, beginning with the physical mapping efforts, continuing through the generation of the high-resolution map and template selection, followed by sequencing and assembly of templates, and concluding with the analysis and dissemination of the sequencing information. The programs that keep track of and display the physical mapping data are nearing completion. The emphasis of the work is shifting now to sequence assembly, editing, and analysis. Another aspect of the work is focused on developing mechanisms to make the data publicly accessible as it is being generated.

The fourth component of the Center is the production effort. A team of about 6 FTE's can currently generate $600-700$ thousand base pairs per year. The goal of the production effort is to maintain this rate as well as to add additional teams in the next few years and increase the productivity of each team.

The final component of the HGC is the biology program, which has been reconstituted to be closely integrated with the overall sequencing effort. The biology effort will play a role in selecting templates beforehand 
LBL is developing into one of the permier centers for the generation of transgenic models for the study of human health and disease. LBL researchers have created two groups of transgenic mice containing human genes. One group expresses high levels of protective forms of lipoproteins, while the other group mimics conditions of human heart disease. The inset figure shows DNA containing the atherogenic apo (a) gene being injected into a mouse embryo. The resulting mouse developed blood vessels clogged with plaque that could eventually cause an aneurysm. The pharmaceutical industry is interested in using such transgenic models for drug development. and then developing biological programs to interpret such a large amount of data in a meaningful way. This program is addressed in the Environmental Assessment and Environmental Impact reports for the Human Genome Laboratory.

Genome Sequencing Initiative Resource Requirements $(\$ M)^{\mathrm{a}}$

\begin{tabular}{lrrrrrrr}
\hline Category & 1995 & 1996 & 1997 & 1998 & 1999 & 2000 & Total \\
\hline Operating & & & & & & & \\
DOE & 7.0 & 8.0 & 8.5 & 8.9 & 9.4 & 9.8 & 51.6 \\
NIH & 3.4 & 8.2 & 10.6 & 14.4 & 16.1 & 13.4 & 66.1 \\
Construction & 0.0 & 0.0 & 0.0 & 0.0 & 0.0 & 0.0 & 0.0 \\
\hline
\end{tabular}

apreliminary estimate of LBL actual year Budget Authority from DOE (B\&R code KP) and $\mathrm{NIH}$.

\section{Transgenic Initiative}

A significant opportunity exists for LBL to expand its transgenic capabilities and leverage the resources associated with the HGC. To fully realize the value of the mapping and sequencing information generated by the HGC, new genes must be identified and their biological function characterized. The genetically engineered mice will clearly play a fundamental role in the discovery of important human genes and the understanding of their function in biology and disease. A Transgenic Initiative is being developed to advance LBL's expertise in generating transgenic animals and enable LBL's Transgenic Facility to better serve the greater scientific community.

The Facility produces transgenic animals for LBL investigators, enabling major studies of gene function in development and disease. The Facility currently constructs animal models for such disease conditions as atherosclerosis, sickle cell disease, thalessemias, Down's syndrome (utilizing DNA provided by the $\mathrm{HGC}$ ), and cancer. Other LBL transgenic projects include the study of such basic biological questions as the control of steroid receptors, RNA editing, and novel interlukin sequences. In parallel with the generation of transgenics, the Facility has developed the capability for inactivating genes in mice through targeted mutagenesis of embryonic stem

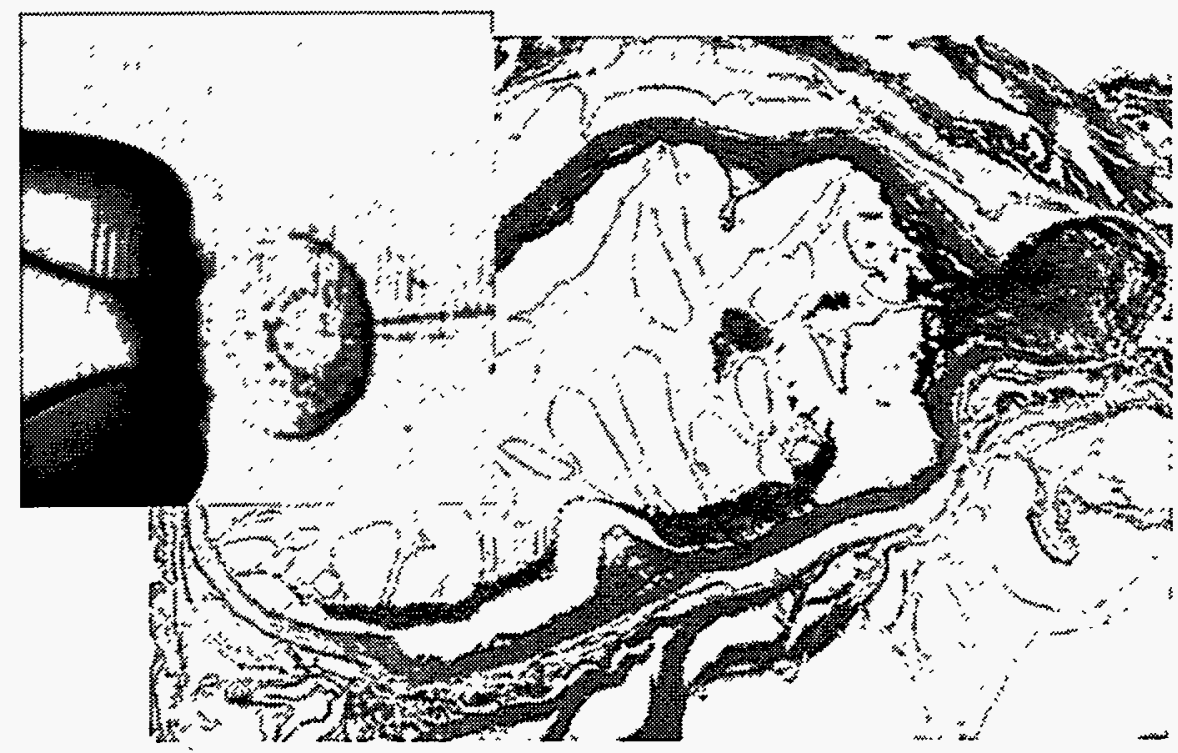


cells. This system has proven effective for the production of germ line chimeras with high frequency. In addition to supplying LBL investigators with these model transgenic animals, the Facility has also, on occasion, made these services available to outside investigators, thus enhancing opportunities for technology transfer with both the industrial and academic communities.

To optimize the relationship between the Facility and the HGC, new technologies need to be pursued. These include: (1) improved construction of in vivo libraries of regions of the human genome in transgenic mice; and (2) methods for creating large targeted deletions in mice of regions syntenic to areas of the human genome being deciphered by the LBL HGC. The development of the Yeast Artificial Chromosome (YAC) system in sequencing and transgenics has provided a technically feasible approach for creating in vivo libraries of large defined segments of the genome. In vivo libraries provide both the ability to study novel phenotypes in the animal as well as enable the complementation of existing mouse mutations. The latter approach allows for the recovery of previously undetermined human homologues for existing mouse mutations.

This initiative will position the LBL Transgenic Facility to serve as one of the premier transgenic facilities. The Facility is ideally set up to provide cost-effective transgenics for investigators who do not possess the capabilities or resources to generate their own transgenic animal models. LBL's transgenic expertise extends beyond mere production of transgenic animals. The Facility possesses a strong instructional component. It provides inexperienced investigators with advice on construct design and preparation as well as instructions for screening and maintenance of the transgenic lines. These discussions provide a rational course for planning well-designed experiments, minimizing the number of DNA constructs and transgenic animals entailed, and ensuring successful results.

Transgenic Initiative Resource Requirements $(\$ M)^{\mathrm{a}}$

\begin{tabular}{lccccccc}
\hline Category & 1995 & 1996 & 1997 & 1998 & 1999 & 2000 & Total \\
\hline Operating & 0.68 & 0.73 & 0.40 & 0.45 & 0.50 & 0.50 & 3.25 \\
Construction & 0.38 & 0.38 & 0.00 & 2.00 & 2.00 & - & 4.75 \\
\hline
\end{tabular}

aPreliminary estimate of actual year Budget Authority (B\&R code KP).

\section{Atomic-Scale Synthesis of Advanced Materials Initiative}

In support of DOE's materials science research programs, LBL is proposing the establishment of a new program to develop techniques for the synthesis, processing, and characterization of materials at the atomic scale. Atomic-scale synthesis will enable highly efficient, finely controlled processing and fabrication for the development of a new realm of materials. In addition, atomic-scale processing can enhance the properties of existing materials, techniques for their synthesis, and their use in conjunction with bulk materials. The effort will strengthen DOE's mission in basic research that supports advanced energy technology, enabling more effective development of energy sources and more efficient processing technology. Atomic- 
An array of CdSe nanocrystals on an amorphous carbon substrate. Each crystallite is highly ordered and faceted. Because of their small size, these crystallites exhibit novel properties that can be exploited in the development of new materials and the enhancement of existing materials.

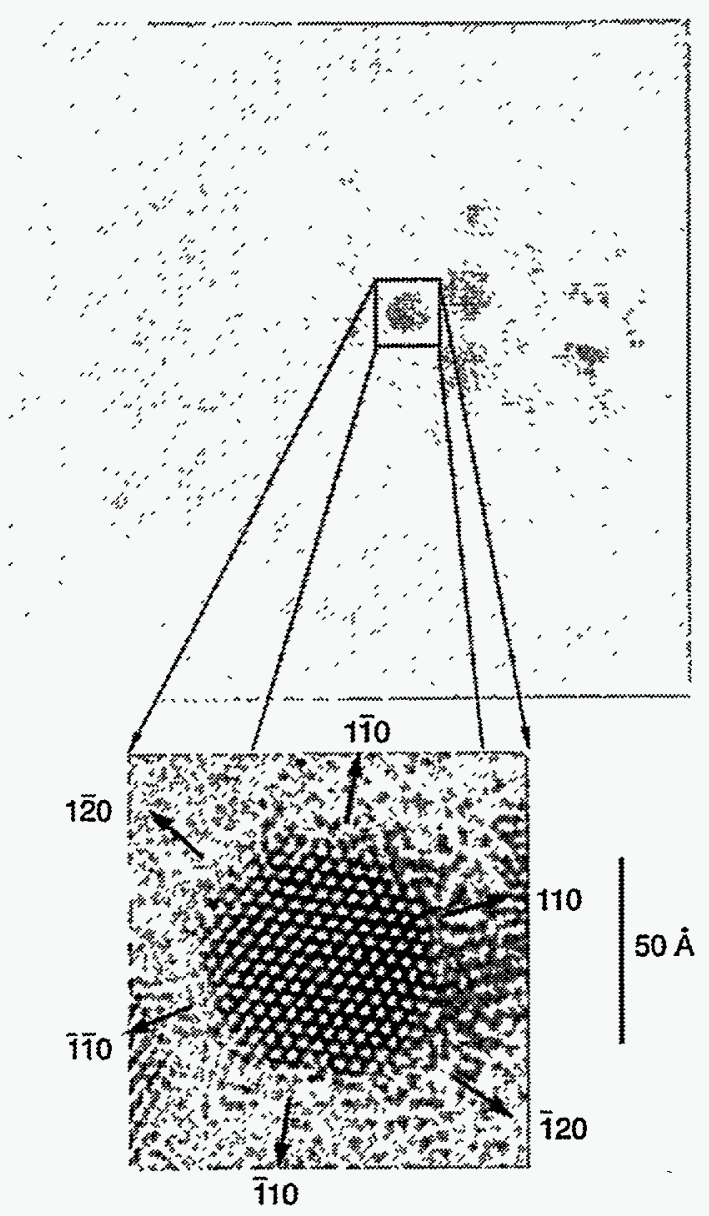

scale control will enable a new generation of materials technology to improve energy efficiency, environmental research, and economic competitiveness, as exemplified below:

- New superconductors and semiconductor structures (energy storage, photovoltaics, sensors, computing, and automation)

- High-energy-product magnets and magnetic films (motors and memories)

- Control of friction (energy dissipation)

- Hard and/or soft coatings (wearing)

- Stronger and lighter structural materials (transport)

- Materials with better heat resistance (energy dissipation)

- Catalysis (coal gasification and liquefaction; environmental cleanup)

The program will extend beyond the current generation of thin films, interfaces, and bulk materials research. These next-generation studies can enable atomically tailored materials optimized for energy storage; energy transfer; and optical, mechanical, electronic, surface (lubricative, adhesive, hardness), and magnetic properties. Optimized characteristics can be achieved through manipulation and deposition of atoms, clusters, and films using novel inorganic and organic synthesis, molecular and ion manipulation, and other techniques, resulting in unique "zero-dimensional" structures 
or quantum dots (points at conventional scales), molecular wires, tubes of one dimension (length), and two-dimensional films of atomic or molecular thickness.

Powerful characterization methods are available at $\mathrm{LBL}$, including the Electron Beam Microcharacterization Facility (see below), advanced laser spectroscopy, and the ALS. This initiative complements the ALS, which will be invaluable for studies of structure (e.g., with glancing-incidence $x$-ray scattering) and other properties. Structural characterization will also be performed by LBL's scanning tunneling microscopes and atomic-force microscopes to characterize hardness, adhesion, and other properties. The research will be conducted under rigorous safety, health and environmental controls, and reviews will ensure compliance with DOE Orders and Occupational Safety and Health Administration (OSHA) regulations. Collaborations with industry will be encouraged, and an industrial visitors' program will be established. As research proposals under this program are developed, they will be reviewed for NEPA and CEQA compliance requirements.

Atomic-Scale Synthesis of Advanced Materials Initiative Resource Requirements (\$M)a

\begin{tabular}{lccccccc}
\hline Category & 1995 & 1996 & 1997 & 1998 & 1999 & 2000 & Total \\
\hline Operating & 5.2 & 5.2 & 5.2 & 5.2 & 5.2 & 5.2 & 31.2 \\
Equipment & 2.0 & 2.0 & 2.0 & 2.0 & 2.0 & 2.0 & 12.0 \\
\hline
\end{tabular}

aEstimate of actual year Budget Authority ( $B \& R$ code KC).

\section{Electron Beam Microcharacterization Facility Initiative}

The Electron Beam Microcharacterization Facility (EBMF) Initiative will support the Department of Energy's Basic Energy Sciences Program by providing state-of-the-art electron beam instrumentation, laboratories and techniques for the characterization of advanced materials at high resolution. The facility will be an essential and integral part of DOE's National Center for Electron Microscopy (NCEM) and as such will be operated as a national user facility. Research at the EBMF will focus on establishing the link between microstructure and properties of solids in order to provide the scientific basis for understanding the behavior of a broad range of advanced materials, important for fundamental science and new technologies. Following the established mode of operation of the National Center for Electron Microscopy, access to the facility will be controlled by an external steering committee representing industry, universities, and government laboratories. As such, the facility will provide essential support to all of DOE's materials research programs in metals, alloys, ceramics, superconducting, semiconducting, and magnetic materials.

The three critical elements of a national research user facility are stateof-the-art instrumentation, a forefront research effort, and a supporting infrastructure. The EBMF is designed to provide essential support for all three elements. The research program at the facility will be centered on electron optical instrumentation and techniques for the microstructural and microchemical characterization of materials at high spatial resolution. A Zcontrast scanning tunneling electron microscope with chemically sensitive imaging capability, and sub-nanometer probe size for high-resolution imaging and diffraction will complement the structural imaging capabilities of the NCEM's One-Ångström Microscope, funded in FY 1994. The capabil- 
The Electron-Beam

Microcharacterization Facility will be a large part of a major upgrade to the National Center for Electron Microscopy. Within this upgrade, NCEM will purchase a new electron microscope with a resolution of 1.7 $\AA$ and a very-high-coherence electron beam. Using a computer image processing method invented at NCEM, the high coherency of the electron beam will be exploited to extend the resolution of the microscope from $1.7 \AA$ to $1.0 \AA$. This microscope/computer combination is called the one-Ångström microscope (OAM). With the microscope, researchers will be able to explore the structures of many technologically important materials at the highest resolution available anywhere, and in a much larger number of viewing directions than with other microscopes, revealing the positions of both metal and light atoms, as shown in the simulation of an $O \AA M$ image of a grain boundary in rutile. ity to obtain spectrum images at nanometer resolution will fill a need critical to many materials programs to identify the structure and composition of nanometer volumes of materials. These capabilities and techniques will be further developed by NCEM scientists and made fully available to researchers in other DOE and industrial research programs.

The EBMF will also provide laboratory space for a medium-voltage instrument, which will supply unique capabilities for in-situ characterization of the dynamic behavior of materials. At $400 \mathrm{kV}$, the instrument will allow samples of representative thicknesses to be observed with minimal radiation damage. The machine will be equipped with a high-speed video camera to record mechanisms and kinetics of transformation, deformation, and reactions in solids. Special stages will be constructed for experiments exploring materials behavior under extreme conditions of temperature, stress, electrical fields, or environmental parameters. In addition to such stages as a hightemperature environmental cell with thin-film diamond windows, there will be provisions to develop specimen holders and instrument configurations for specialized, narrowly focused experiments such as an in-situ diamond anvil cell for high-pressure studies or an in-situ nano-indenter for studies of friction, wear, fatigue and early stages of deformation. A supporting laboratory will be dedicated to simple microlithography for novel sample geometries. Research with this facility will support different materials research efforts within DOE's Basic Energy Sciences Program.

Sufficient laboratory space has been included to install supporting instrumentation, prepare samples, perform thermomechanical treatments,

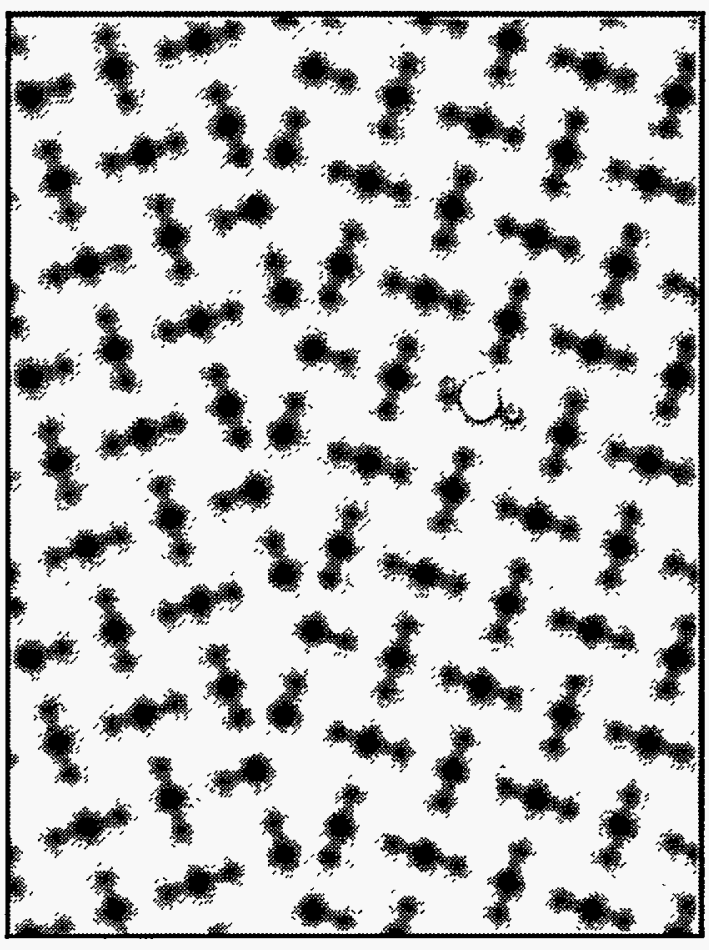
make measurements and pre-characterize materials before examination by advanced electron optical techniques. Additional laboratory space will be important for industry, university, and government scientists who need to finalize experiments or sample preparation as well as for resident scientists to perform materials research prior to electron beam microcharacterization. In addition to laboratory space and equipment, the EBMF will provide facilities for workshops to educate industrial and university scientists and professionals in electron beam characterization, theory, computing, and sample preparation. The facility will also allow the NCEM to lead the materials characterization community in topical discussion meetings and workshops focused on forefront developments in the field. It is anticipated that an Environmental Assessment and Initial Study would be prepared for this facility in compliance with NEPA and CEQA. 
LBL, through its Accelerator and Fusion Research Division, is collaborating with SLAC and LLNL in the design and construction of PEP II, an asymmetric B Factory at Stanford Linear Accelerator. The concept was developed by Pier Oddone, Deputy Director of $L B L$. The Physics Division is part of a broad international collaboration developing the detector for the project, $B A B A R$, shown in the figure. $L B L$ has several roles in the development of the vertex detector, including data acquisition, particle identification, and on-line and offline computing.
Electron Beam Microcharacterization Facility Initiative Resource Requirements $(\$ M)^{\mathrm{a}}$

\begin{tabular}{lccccccc}
\hline Category & 1995 & 1996 & 1997 & 1998 & 1999 & 2000 & Total \\
\hline Operating & 0.0 & 0.0 & 3.0 & 4.4 & 0.0 & 0.0 & $7.4^{\mathrm{b}}$ \\
Equipment & 0.0 & 0.9 & 4.7 & 2.3 & 0.6 & 0.0 & 8.5 \\
\hline
\end{tabular}

apreliminary estimate of LBL actual year Budget Authority (B\&R code KC).

${ }^{b}$ Amount representing equipment obligations.

\section{B Factory at Stanford Linear Accelerator Initiative}

The study of B-meson decays will be one of the key elements of the worldwide high-energy physics program for many years to come. These studies are limited today by the relatively low rate of events produced at $\mathrm{e}^{+} \mathrm{e}^{-}$storage rings. To study the most interesting processes within the Standard Model, both rate decays and, even more important, the phenomenon of charge conjugation-parity (CP) violation, an effective increase in the event rate of at least a factor of 100 is required. In the B Factory, this will be accomplished by increasing the luminosity by a factor of 10 and by simultaneously increasing the event sensitivity through the use of asymmetric collisions (equivalent to another factor of 10 in luminosity), as described below.

The concept of using asymmetric collisions of storage-ring beams with a center-of-mass energy at the Upsilon (4S) resonance was originally suggested at LBL. The Upsilon (4S) decays into two B mesons nearly at rest in

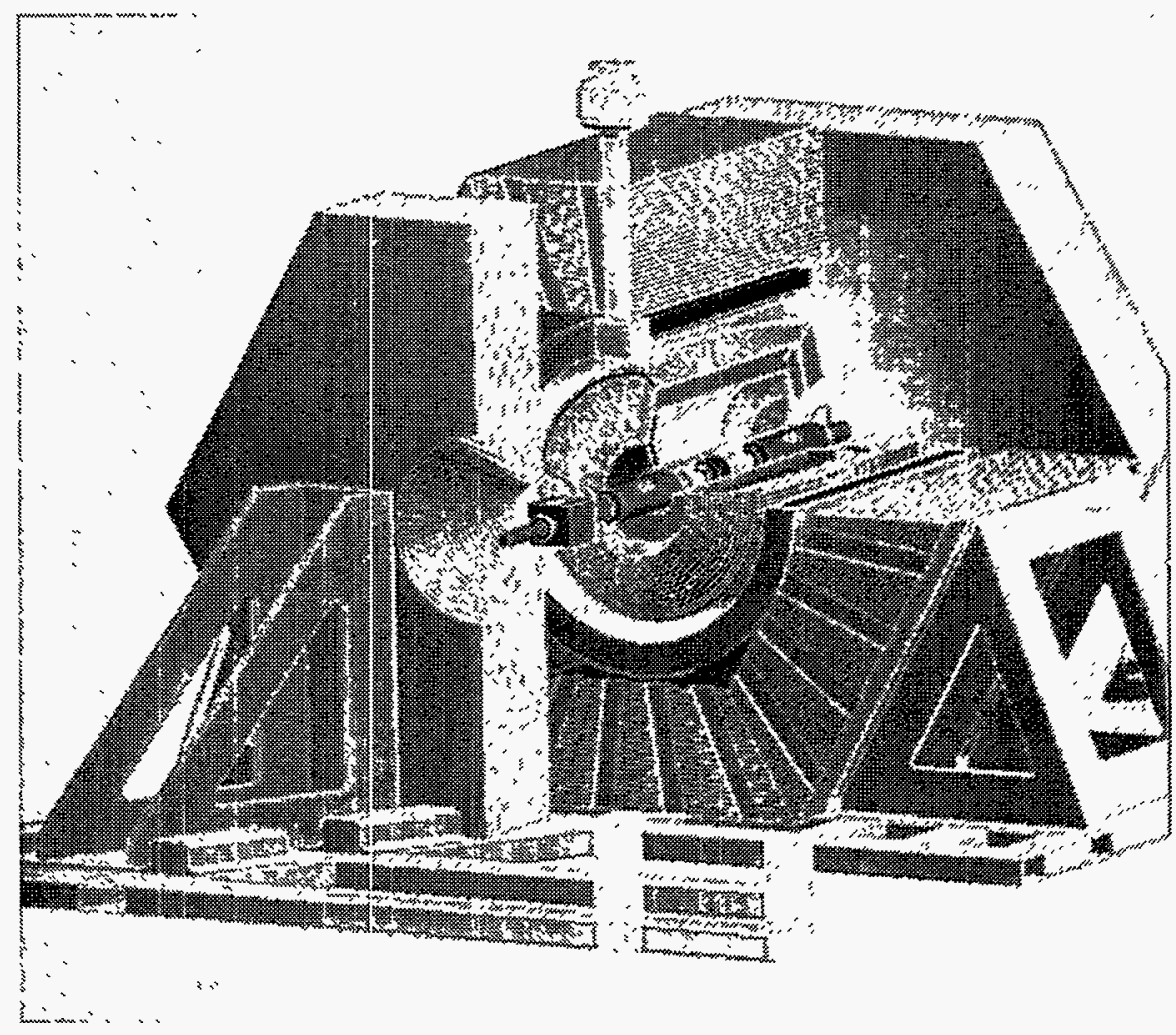


the center of mass. Since the center of mass would be moving in the laboratory frame (because of the different beam energies of the two rings), the two $B$ mesons move along the direction of the higher-momentum beam, and their decays are separated in space (or equivalently, time). This separation permits the reconstruction of the individual $B$ mesons and the study of the time evolution of their decays. As noted, the use of asymmetric collisions is equivalent to an additional factor of approximately 10 in luminosity for the study of the most interesting channels for $\mathrm{CP}$ violation.

LBL, in collaboration with the Stanford Linear Accelerator Center (SLAC) and Lawrence Livermore National Laboratory (LLNL), has evaluated the use of the Positron-Electron Project (PEP) storage ring in conjunction with a new low-energy storage ring. A B Factory based on PEP, which serves as the high-energy, 9-GeV ring, with the addition of a new low-energy 3.1-GeV ring, is attractive both scientifically and fiscally. A conceptual design, prepared in collaboration with SLAC and LLNL, has shown that such an asymmetric B Factory scenario is entirely feasible with state-of-the-art technology. After extensive joint review by DOE and the National Science Foundation (NSF), and comparison with a competing B Factory proposal from Cornell University, President Clinton selected SLAC as the site for the U.S. B Factory project.

Construction of the $\$ 177$ million PEP-II accelerator began in January 1994 and will extend through the end of FY 1998. The project is being carried out as a collaboration of SLAC, LBL, and LLNL. LBL construction activities focus mainly on the Low-Energy Ring. In particular, LBL is responsible for fabrication of the Low-Energy Ring arc magnets (roughly 200 dipoles, 300 quadrupoles, 150 sextupoles, and 200 corrector dipoles) and some of the key "special" magnets in the interaction region. We are also responsible for fabrication of the arc vacuum system (about $1500 \mathrm{~m}$ of vacuum chambers) and for a portion of the PEP-Il power supplies (corrector supplies and high-power, non-chopper power supplies). Staging and fabrication space sufficient to serve the PEP-II project has been identified on site. LBL has played a key role in the development and testing of the innovative multibunch feedback systems and will be responsible for providing the transverse feedback systems for both PEP-II rings. LBL accelerator physicists and engineers continue to play key roles in the B Factory project, including serving as Deputy Project Director, and as System Manager, and System Engineer for the entire Low-Energy Ring. In FY 1998, LBL personnel will play a leading role in the commissioning of the Low-Energy Ring.

LBL will have a major role in the design and construction of the silicon vertex tracker for the B Factory. This is an especially critical component of the detector beçause it is essential to determining the location of the $B$ decay vertices. LBL's effort builds on its extensive experience with the SVX in the Collider Detector at Fermilab (CDF), with the major effort accomplished for the Solenoidal Detector Collaboration (SDC), and with the design of the D-Zero upgrade planned at Fermilab. The silicon vertex tracker will require substantial contributions from the Engineering Division, as well as from the Physics Division itself.

LBL will, in addition, assume responsibility for other components of the B Factory detector. Particle identification is a particularly important challenge since it is essential to determining the particular decay modes being observed. While Cerenkov radiation is likely to be a basis for particle identification, it is not yet certain exactly how it will be employed. Addi- 
tional information may come from time-of-flight and from $\mathrm{dE} / \mathrm{dx}$ measurements. LBL's experience from the time projection chamber (TPC) and Mark II will be a valuable resource in this work.

LBL will also undertake a substantial role in electronics for the B Factory detector. This effort will build on the tremendous capability established, in conjunction with the Engineering Division, to meet the daunting requirements of the SDC, which we were designing for the Superconducting Super Collider (SSC). Similarly, data-acquisition work, originally designed in part for the SSC, gives us a strong basis for tackling the problems of the B Factory detector.

The B Factory detector will have enormous computing needs, and LBL will be a leader in designing the overall architecture for this work, with leadership coming from the Information and Computing Sciences Division.

A NEPA and CEQA review will be performed for activities proposed to take place at $L B L$ under this initiative.

B Factory at Stanford Linear Accelerator Initiative Resource Requirements $(\$ M)^{a}$

\begin{tabular}{lrrrrrrl}
\hline Category & 1995 & 1996 & 1997 & 1998 & 1999 & 2000 & Total \\
\hline Operating & 2.5 & 2.7 & 2.7 & 2.5 & 2.5 & 2.5 & 15.4 \\
Construction & 10.0 & 7.0 & 5.0 & 1.0 & - & - & $25.0 \mathrm{~b}$ \\
\hline
\end{tabular}

aEstimated LBL outlays for accelerator and detector R\&D. Subject to project review on semiannual basis.

bincludes prior year (FY 1994) construction funds.

\section{Large Hadron Collider Initiative}

CERN's Large Hadron Collider (LHC) is destined to dominate highenergy physics for many years after its completion between 2002 and 2005. LBL is working to secure major roles in both accelerator work and in the ATLAS detector. The accelerator work is being coordinated with efforts at Brookhaven National Laboratory (BNL) and Fermilab. LBL's expertise in superconducting magnets, and superconducting cable in particular, is sure to be important to the project. In addition, LBL will undertake design and fabrication of the liner, which will ensure that the high vacuum in the beam pipe is not ruined by desorption caused by synchrotron radiation.

An LBL team, drawn in large part from the SDC effort, will play an important role in charged-particle tracking for the ATLAS detector. This will involve both silicon strip detectors and pixel devices. The latter will be developed using the Physics Division's Microsystems Laboratory. For the pixel work, LBL expects to design and fabricate pixel-detector elements, ondetector readout electronics, and off-detector control and readout electronics. We will contribute to the software and to the mechanical design. We may actually construct a portion of the full pixel structure at LBL.

In collaboration with UC Santa Cruz, LBL will design, fabricate, and test a significant portion of the silicon tracker for ATLAS, including the rad-hard front-end electronics. In collaboration with both UC Santa Cruz and UC Irvine, LBL will work on the data-acquisition and control electronics for the silicon strip readout. In collaboration with UC Irvine, LBL will have a role in the ATLAS data-acquisition system and in the level-2 trigger.

The major LBL accelerator contributions will be in three areas: vacuum 


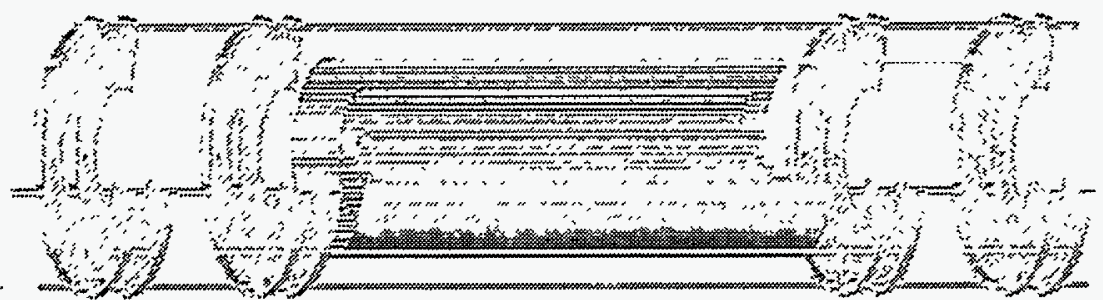

LBL, through its Accelerator and Fusion Research Division, is collaborating on the design and fabrication of the Large Hadron Collider at CERN, with programs in vacuum systems, superconducting magnets and cables, and feedback systems. The Physics Division is playing a leading role in the design and fabrication of the inner tracking system, which includes a silicon strip tracker and pixel tracking system, shown in the figure.

system, interaction-region quadrupole magnets, and beam feedback and control. The vacuum system contributions include (1) fabrication of beam tube liners, including the development of prototypes and the testing of fullscale liners; (2) oversight of full-scale production of beam tube liners (approximately $100 \mathrm{~km}$ for both beams); (3) development of demountable flanges and Be vacuum chambers for the interaction regions; (4) fabrication of beam dump vacuum chambers; and (5) development of a vacuum barrier for the cryostat insulation system.

Development of interaction-region (IR) quadrupoles is a joint task with Fermilab National Accelerator Laboratory (FNAL). LBL will work with FNAL on the development of a conventional IR quadrupole design, which FNAL will scale up and fabricate as full-scale prototypes. LBL will then develop an advanced $I R$ magnet design, based on an improved superconductor also being developed at LBL. One-meter-long prototypes of these magnets will then be fabricated at LBL. This advanced IR quadrupole may replace the conventional quadrupole. LBL will also provide cabling technology and strand-coating technology to the LHC project.

\footnotetext{
LBL will perform analysis, design, and fabrication work on the beam feedback system needed to stabilize the central beam motions in the LHC. The technologies needed are similar to those being developed at LBL for the B Factory system, but are more challenging due to the need to preserve beam density in the absence of radiation damping. A NEPA and CEQA review will be performed by LBL for activities proposed to take place at LBL under this initiative.
}

Large Hadron Collider Initiative Resource Requirements $(\$ M)^{\mathrm{a}}$

\begin{tabular}{lcccccrc}
\hline Category & 1995 & 1996 & 1997 & 1998 & 1999 & 2000 & Total \\
\hline Operating & 2.1 & 5.7 & 6.8 & 7.0 & 6.6 & 5.1 & 33.3 \\
Construction & 0.0 & 0.3 & 2.5 & 5.8 & 17.1 & 21.9 & 47.6 \\
\hline
\end{tabular}

aPreliminary estimate of LBL actual year Budget Authority (B\&R code KA). 


\section{Relativistic Heavy-Ion Collider Initiative}

LBL played a seminal role in defining relativistic heavy-ion physics and continues to maintain its leadership role. The Relativistic Heavy-lon Collider (RHIC) is now under construction at BNL, and LBL is the lead laboratory for the first approved RHIC experiment, the Solenoidal Tracker at RHIC (STAR) detector. LBL's Relativistic Nuclear Collisions Group is providing a focus for these RHIC activities. With 47 physicists, engineers, and technicians (including the spokesperson and project director) from LBL working on this experiment, the STAR collaboration now consists of over 350 physicists and engineers from 35 institutions internationally.

The picture shows the support structure for the construction of the outer field cage of the time projection chamber (TPC) to be built for the STAR Detector of the Relativistic Heavy-Ion Collider. The outer field cage and other components of the TPC need to use minimal materials yet be structurally strong. The TPC is a large-volume device ( 2 meters in diameter by 4.1 meters in length). It will serve as the central detector in $S T A R$, and is designed to reconstruct as completely as possible the final state of particles after a gold-on-gold ion collision at $40 \mathrm{GeV}$.

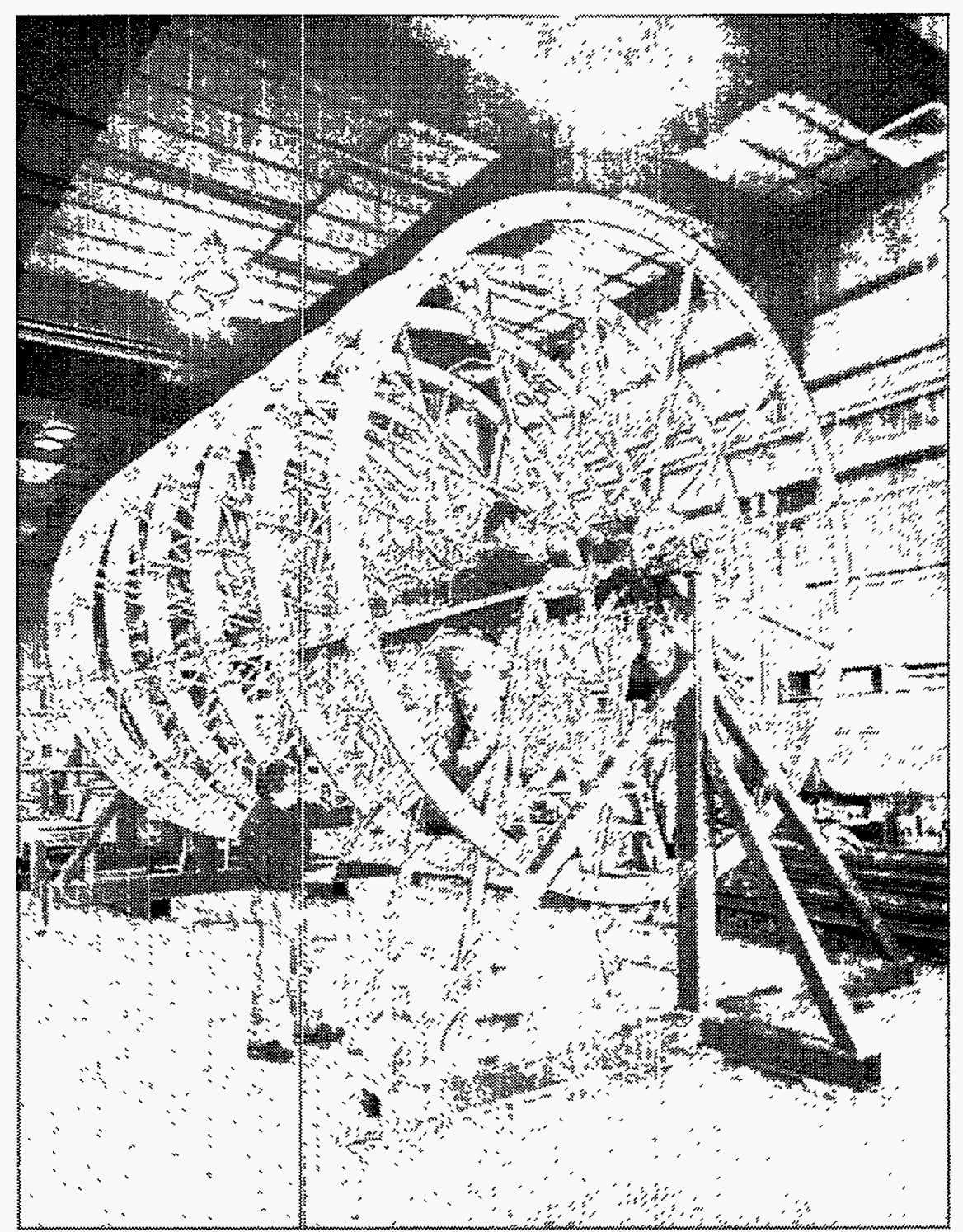

The goal of the experiment is to study particle production at midrapidity on an event-by-event basis to identify the phase transition from normal nuclear matter to quark matter. An event-by-event measurement of the produced particles at midrapidity provides the opportunity to select on events with extreme values of temperature (particle spectrum), flavor 
(strangeness content), shape (particle momenta), and size (two-particle correlations). Furthermore, the study of high-pt particle production as a function of energy and mass of the colliding system may also be an attractive experimental approach to identify the presence of quark matter.

The experiment will consist of a TPC and a silicon vertex tracker located inside a solenoidal magnet to provide tracking, momentum analysis, and identification of charged particles; a time-of-flight array to extend the particle identification to higher momentum; TPCs to provide tracking at large pseudo-rapidities; an electromagnetic calorimeter; and a multilevel flexible trigger. Projects at $L B L$ now receiving support from $R H I C$ detector R\&D funds administered by BNL include TPC improvements for measurements at the high track densities anticipated at RHIC, integrated electronics for advanced detectors, and development of a high-bandwidth data-acquisition system. Research is also proposed in the area of heavy-ion beam cooling for improved luminosities at the collider. These projects and the proposed STAR program are being developed in compliance with DOE Orders and all other applicable ES\&H requirements. LBL expects this R\&D effort and the detector engineering to continue over the next few years.

Relativistic Heavy-Ion Collider Initiative Resource Requirements (\$M) ${ }^{\mathrm{a}}$

\begin{tabular}{lccccccc}
\hline Category & 1995 & 1996 & 1997 & 1998 & 1999 & 2000 & Total \\
\hline Operating & 3.1 & 4.0 & 4.5 & 5.0 & 5.0 & 5.0 & 26.6 \\
Construction & 4.5 & 3.4 & 2.1 & 1.0 & 1.0 & 1.0 & 13.0 \\
\hline
\end{tabular}

apreliminary estimate of LBL Budget Authority (FY 1994 dollars), including equipment (B\&R code KB).

\section{IsoSpin Laboratory Initiative}

A broad base of nuclear scientists has recommended that the North American nuclear physics community should pursue the construction of a dedicated, flexible, radioactive nuclear beam facility. It would provide intense beams of nearly all elements for a program of scientific studies in nuclear structure, nuclear reaction dynamics, astrophysics, high-spin physics, nuclei far from stability, materials and surface science, and atomicand hyperfine-interaction physics. This international initiative has tentatively been given the name IsoSpin Laboratory (ISL) to underscore the key feature of this new physics tool.

The advent of unstable, radioactive nuclear beams in this decade may rival in importance the development of heavy-ion beams in the 1960s. These beams remove the restraint imposed by the natural neutron-to-atomicweight ratio of stable beams upon nuclear, astrophysical, atomic, and materials science experiments, and add the new dimension of isospin to the two traditional dimensions of nuclear spin and temperature.

Elsewhere in the world, facilities utilizing the fragmentation of heavyion projectiles are in operation and others are planned. They produce radioactive nuclear beams in the energy range of tens of $\mathrm{MeV} /$ nucleon to $\sim 1000 \mathrm{MeV} /$ nucleon. For the new North American project, a complementary approach to radioactive beam production has been chosen. The ISL is based on the coupling of two accelerators: the first to deliver a high-current light-ion beam to a thick spallation or fission target and the second to accelerate the emanating radioactive species with high efficiency and excellent beam qualities. 
Several options for implementing the ISL are being studied. One of the most attractive seems to be a rapid cycling synchrotron to serve, together with a new 70-100 MeV linac injector, as the primary beam accelerator. A proton energy of $1 \mathrm{GeV}$ and a current of $200 \mu \mathrm{A}$ would be obtainable, fully meeting ISL requirements. This would be followed by several target stations and a heavy-ion linac for the post-acceleration of the radioactive beams.

The ISL offers exciting physics prospects leading to a new understanding of nuclear structure and reaction dynamics, astrophysics, materials science, and atomic physics. LBL expertise in the underlying physics and machine design and development are coupled with excellent site characteristics and infrastructure. Existing expertise in environmental protection, health, and safety for handling beam systems and isotopes also couples well to the facility. Budget planning for the facility is under way; the following costs are for an initial design and planning program. It is anticipated that either an environmental assessment or an environmental impact statement will be required under NEPA and that an environmental impact report will be required under CEQA.

IsoSpin Laboratory Initiative Resource Requirements $(\$ M)^{\mathrm{a}}$

\begin{tabular}{lccccccr}
\hline Category & 1995 & 1996 & 1997 & 1998 & 1999 & 2000 & Total \\
\hline Operating & 2.0 & 2.5 & 3.5 & 3.5 & 3.5 & 3.5 & 18.5 \\
Construction & 0.0 & 0.0 & 0.0 & 0.0 & 0.0 & 0.0 & 0.0 \\
\hline
\end{tabular}

abudget to be determined for the facility.

\section{National Information Infrastructure: Networking Initiative}

The primary goal of the Networking Initiative is to enhance national research and domestic economic development through high-speed, advanced computation and data-communications capabilities. This initiative responds to the need for advanced information highways that can meet America's information needs. It addresses the critical relationship between successful federal scientific research and enabling high-performance computing and communications. The initiative stresses the importance of the U.S. computer industry to the national economy.

The DOE and LBL programs place emphasis on major scientific initiatives, including the development of software tools, new computational capabilities, and gigabit data-transmission network applications. As part of the DOE program, LBL has an R\&D strategy based on developing an integrated, distributed computing environment, including advanced workstation capacity, computer servers, database servers, and a high-speed network integrated through a software bus environment.

LBL is developing key technologies as a part of its high-performance computing research program, including: high-speed networking, advances in the software bus, development of scientific databases, imaging and visualization tools for multimedia capacity, a video-audioconferencing tool (VAT), "light-weight sessions" that permit the simultaneous multicast of and access to multiple video and audio conferences without extensive prearrangement and setup, development of a prototype workstation-based "electronic whiteboard," deployment of routine video conferencing over the Energy Sciences Network (ESnet), creation of a new audiodriver for Sun 
Microsystems' operating system, research on network traffic data and patterns, and analysis and control of gateway congestion.

These tools are being applied to Grand Challenges, including resolution-limited imaging (in the areas of medicine, structural biology, geology, and the ALS imaging systems), global climate modeling, human genome research, and development of the next generation of detectors for nuclear and high-energy physics.

National Information Infrastructure: Networking Initiative Resource Requirements $(\$ M)^{a}$

\begin{tabular}{llllllll}
\hline Category & 1995 & 1996 & 1997 & 1998 & 1999 & 2000 & Total \\
\hline Operating & 1.57 & 1.65 & 1.74 & 1.82 & 1.91 & 2.00 & 10.69 \\
Construction & 0.0 & 0.0 & 0.0 & 0.0 & 0.0 & 0.0 & 0.0 \\
\hline
\end{tabular}

aPreliminary estimate of LBL actual year Budget Authority (B\&R code KC).

\section{Basic Energy Sciences Program Direction}

\section{Materials Sciences}

LBL is one of the world's leading centers of research in materials science and the chemistry and physics of materials that are important to both the production and efficient use of energy. In addition, outstanding programs exist in advanced energy projects, in engineering and geosciences, in biological energy research, and in applied mathematics. Several of these programs are expected to expand, as indicated below.

Basic Energy Sciences (BES) programs in materials sciences will continue to emphasize new and forefront research projects for the synthesis, processing, and characterization of advanced materials. Expanded areas include the design and construction of synchrotron-radiation instrumentation and its application to important programs in materials sciences and advanced materials synthesis and fabrication technologies, especially at the atomic scale. Leading programs continue in $x$-ray optics, electron microscopy, solid-state physics, surface sciences, catalysis, polymers, biomolecular materials, metallurgy, electrochemical materials, electronic materials (including super-and semiconductors), ceramics, and materials chemistry. The ALS, a third-generation synchrotron-radiation facility, produces the world's brightest beams of ultraviolet and soft x-ray radiation. The commissioning of the injection system and storage ring has been completed. The ALS, Center for Advanced Materials (CAM), Center for X-Ray Optics (CXRO), and National Center for Electron Microscopy (NCEM) are organized interdisciplinary research centers that bring DOE resources to bear on scientific challenges of national importance.

In support of the ALS, the Laboratory is conducting research on storagering physics and engineering, ultrahigh-vacuum technology, instrumentation and feedback systems, insertion devices, beamline optical systems, and magnet systems. At CXRO, research is conducted on advanced opticalsystem components for the utilization of high-brightness photon beams.

CAM will continue major research efforts selected because of their potential impact on U.S. industrial strength. The research focus evolves with new discoveries and with the changing needs of U.S. industry for fundamental research to underlie its development and commercialization 
activities. Areas of specific focus are: surface science and catalysis; polymers and composites; high-performance metals; ceramics science; biomolecular materials; enzymatic synthesis; and high- $\mathrm{T}_{\mathrm{C}}$ superconductivity. These areas are discussed in further detail in the Industrial Competitiveness subsection of this section.

NCEM, a national user facility, is supported by the DOE Metallurgy and Ceramics Program but contributes substantially to research in other fields, such as biology and geology. The heart of NCEM consists of two microscopes: the High-Voltage Electron Microscope, the most powerful microscope of its kind in the U.S., and the Atomic-Resolution Microscope, with a resolution of $1.5 \AA$-currently the highest resolution in the U.S. To maintain U.S. leadership in electron microscopy, we are proposing to enhance the Center through the design and acquisition of new state-of-the-art microscopes.

Research at NCEM is carried out on a wide range of materials, including studies of high-temperature superconducting materials, structural materials, magnetic materials, ceramics, and amorphous silicon semiconductor materials. Research on the structure and properties of transformation interfaces has the goal of determining the atomic configuration at structural boundaries and the relationship between structure and properties at the interface.

LBL participates in the DOE Center of Excellence for the Synthesis and Processing of Advanced Materials. The Director of the Materials Sciences Divișion is the national coordinator for the focus area on Nanoscale Materials for energy applications, one of seven focus areas in the Center. This area of research has attracted the interest of a number of federal agencies, and LBL is hopeful that its research program can, with appropriate collaborative infrastructure and operating support, make a major contribution to the field. Materials Sciences Division investigators also lead projects in the other six focus areas: Metal Forming, Materials Joining, Hard Magnets, Surface Hardness, Corrosion Coatings, and Polymers.

Several communities of users in the physical, chemical, and biological sciences and in materials and nuclear engineering have expressed their need for a pulsed, megawatt-class neutron source that is based on an accelerator and a production target. The DOE Office of Basic Energy Sciences has asked LBL to host a Spallation Neutron Source Design Study to examine the users' needs and available technology concepts and to produce a conceptual design. LBL offers the accelerator expertise necessary to contribute to and manage the design effort and will not be in the competition for the neutron facility itself.

\section{Chemical Sciences}

DOE's Chemical Sciences Program supports focused research in several LBL divisions. Efforts in the Chemical Sciences Division emphasize chemical physics, dynamics and mechanisms of chemical reactions and combustion processes, catalysis, electron spectroscopy, atomic physics, photochemistry, theoretical chemistry, and chemistry of the actinide elements and their relationship to environmental issues. Programs in the Energy and Environment Division focus on advanced combustion and the mechanisms for minimizing emissions and improving fuel efficiency. In the Structural Biology Division, programs in photochemistry and the chemistry of electronically excited molecules are being conducted. In the Nuclear Science Division, a study of the chemical properties of the heaviest elements is being 
conducted. In the Physics Division's mathematical department, a study of hydrodynamical computational models for combustion processes is being carried out. This research is directed toward developing high-accuracy techniques that take into account both turbulent flow and the chemical interactions of these combustion processes.

The extensive chemical physics research includes several major programs. One focuses on spectroscopic studies of the structures of reactive intermediates. Laser-induced fluorescence, multiphoton ionization, and negative-ion photodetachment are used to study reactive species such as free radicals and cluster ions that may be important in combustion processes, reactive plasmas, and high-technology manufacturing processes. Techniques such as the use of crossed molecular beams are used for advanced and novel studies of the dynamics of important reactions with the goal of understanding elementary chemical reactions through singlecollision conditions or laser excitation. This research on reaction dynamics and combustion is expected to grow during the next several years.

The program in reactivity at surfaces and interfaces will involve molecular studies of interfacial phenomena using new techniques in laser spectroscopy and $x$-ray scattering. The program is designed to gain an understanding of chemical reactivity in key areas of energy science, including nearly all catalytic reaction systems, solar-energy conversion technologies, lightassisted chemical syntheses, electrochemical energy-conversion technologies, and corrosion phenomena.

Chemical energy research at $L B L$ has revealed new reactions between transition metals, such as rhenium, and organic molecules that are important to the improvement of catalysis involved in coal-conversion processes.

Continuing program areas focus on the fundamental chemistry of important environmental and fuel species, including aqueous and gaseous species of carbon and sulfur. Catalytic conversion of carbon monoxide and hydrogen to gaseous and liquid fuels is studied to develop more efficient catalysts for hydrocarbon production.

The research programs in theoretical chemistry have the goal of accurately predicting chemical reaction dynamics, especially those that are too complicated to be solved experimentally. The program on photochemical and radiation sciences includes research into the photochemistry of materials in the stratosphere (with applications to the role of trace gases in the "greenhouse effect").

Research in the actinide chemistry program in the Chemical Sciences Division has two thrusts: (1) the design and synthesis of sequestering agents for treatment of actinide poisoning and for possible application to spent reactor fuels, and (2) the preparation and study of new compounds incorporating actinides. A program to investigate the chemical properties of the heaviest elements ( $Z$ of 102 to 106$)$ at the furthest reaches of the periodic table is being undertaken in the Nuclear Science Division.

Research in the energy and environment area includes theoretical and experimental programs on ignition, reactivity, turbulence, and energy transfer in combustion systems. Advanced approaches include studies of photodissociation, laser spectroscopy methods, molecular-beam mass spectroscopy, and the use of unimolecular kinetics for the theoretical study of high-temperature reactions important to combustion. Another area of research is laser-material interactions for chemical analysis.

Research in structural biology is directed at a fundamental understanding of the chemistry of electronically excited molecules, with attention on 
features that relate to the conversion of photon energy and the photoassisted synthesis of high-value compounds. Projects focus on the manganese catalytic function in artificial photosynthesis, the photoinduced reduction of $\mathrm{CO}_{2}$ into organic products, and polyelectrolyte interfaces for increasing quantum efficiency in photosynthetic processes. Other work explores chemistry induced by red and near-infrared light, the most abundant form of energy available from the sun. The focus is on redox reactions in solution that may lead to new concepts for chemical storage and conversion of these long-wavelength quanta into electricity and on their use for controlled photochemical synthesis in a solid matrix. A major new effort is directed at monitoring elementary reaction steps in these environments by timeresolved Fourier-transform infrared spectroscopy.

\section{Applied Mathematics and Computer Science}

The program in mathematics at LBL centers on the development of numerical and analytical methods and their application to the most challenging problems in physics and engineering. Investigations that were started within the LBL Mathematics Department have been at the frontiers of such topics as vortex methods, random-choice techniques, high-resolution methods in gas dynamics, front-propagation techniques, and lattice and polymeric models in turbulence.

The five most active areas are: vortex and particle methods, interface techniques, the statistical mechanics of vortex systems, the parallel implementation of codes for large-scale scientific computing, and fast algorithms. In the first area, recent successes include the design and experimental validation of vortex codes for three-dimensional turbulent flow around complex bodies, rapid implementation of particle methods, and numerical studies of engineering flows and of suspensions. In the second area, we develop and apply state-of-the-art aigorithms, such as the level set methodology and new surface integral techniques for following free surfaces. These techniques allow models of complex problems in crystal growth and dendrite solidification, minimal-surface and capillarity theory, two-phase flow, and flame propagation. In the third area, we are implementing renormalization schemes for vortex interactions, have undertaken a systematic analysis of vortex motion in constant temperature systems, and are applying it to problems in superfluidity. In the fourth area, we are one of the leaders in the practical implementation of highly sophisticated codes for realistic problems on massively parallel processors. In the fifth area, we are developing and implementing new fast algorithms for elliptic and parabolic, linear and nonlinear problems.

The Scientific Data Base Management Research Program will continue to investigate new data-management techniques suited to scientific and statistical applications. New requirements arise from the structure of some scientific data (e.g., sparse multidimensional tables, temporal data) and operation needs (e.g., transposition, aggregation, random sampling, proximity searches). Thus, new efficient techniques for data-storage organization, new algorithms for data manipulation, and new data-modeling methods to improve the semantics of scientific data are being developed.

The Supercomputing Access Tools Program addresses the problems of scientific computing in distributed environments, with the goal of developing techniques that will partition the computational requirement optimally across distributed resources. The research on a software bus system will 
With the support of the Nuclear Physics program in DOE's Office of Energy Research, $L B L$, in partnership with other national laboratories, is constructing and operating the Gammasphere as a national user facility. This instrument for studying nuclear structure was first operational in $F Y$ 1993 in an early-implementation mode with 30 of the eventual 110 Ge detectors. Since then, experiments, conducted by researchers from around the world, have included studies of new superdeformed bands in nuclei, nuclear symmetries, identical bands in different nuclei, and pairing correlations in superdeformed nuclei. The photograph shows the assembling of the final framework for holding the 110 detectors. The detector is to be completed this coming year. result in an ability to generate interoperable, and therefore reusable and replaceable, software. This will greatly enhance the computing environment available to energy research scientists. Visualization and imaging tools compatible with this innovative architecture will be developed.

A new area of search, in collaboration with the Xerox Palo Alto Research Center, has been in multimedia conferencing. New protocols have been developed to support high-quality audio and video conferencing. New protocols also have been developed to support high-quality audio and video transmission on the Energy Sciences Network and other parts of the Internet. In addition, work is being done to incorporate an interactive "whiteboard," or graphic display window, in which network users can make real-time modifications.

\section{Nuclear Physics Program Direction}

Nuclear physics research at LBL will continue to focus on the experimental and theoretical investigation of the structure and properties of nuclei, emphasizing studies of nuclei under extreme conditions (of temperature, isospin, angular momentum, and density, for example). The research programs also play an important role in the education and training of young scientists. In addition, technology development efforts are directed toward ongoing detector design and construction, often in collaboration with industrial partners.

LBL has played a seminal role in defining the forefront of relativistic heavy-ion physics since the field's inception and intends to continue to maintain this leadership role. The main focus of the future high-energy, heavy-ion research program at LBL is RHIC, which is currently scheduled to be completed at BNL in 1999.

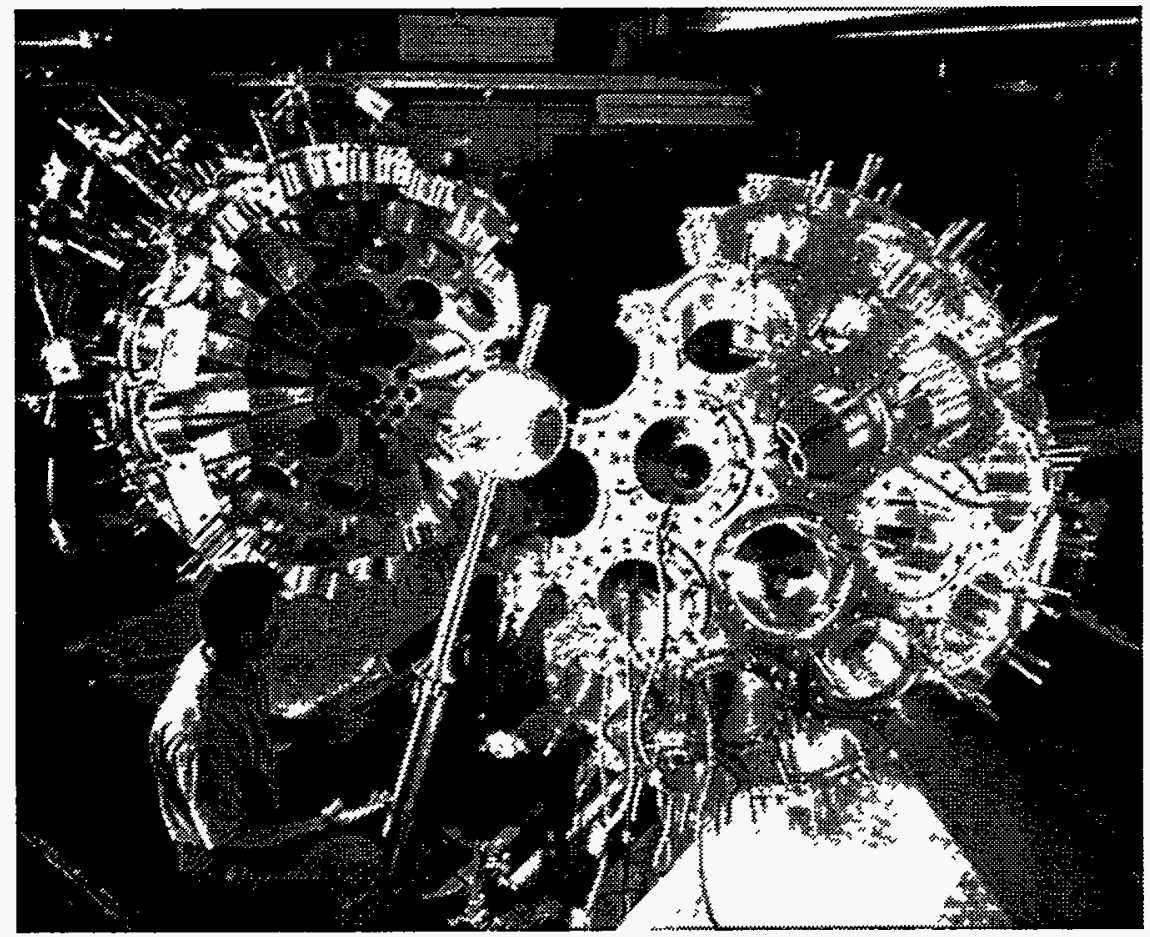
Experiments studying the collision of heavy ions are being carried out in four energy regimes: (1) the Bevalac (now shut down, but data analysis continues), where nuclear matter is compressed sufficiently to study its equation of state; (2) the AGS at BNL, extending the studies of the Bevalac to an energy range where the maximum pressure from the baryons is likely to occur; (3) the super proton synchrotron (SPS) at CERN, where the energy density of the nucleons in the collision of very heavy nuclei may be sufficient to produce a phase transition to a plasma of free quarks and gluons; and (4) RHIC, where the energy density of the produced particles will be sufficiently high that production of the quark-gluon plasma is expected to occur. Understanding the reaction dynamics and the nuclear matter 
equation of state is of fundamental interest and necessary for a physical description of neutron stars and supernova explosions. It is believed that the quark-gluon plasma existed soon after the Big Bang at the creation of the Universe and may exist now in the cores of neutron stars.

The broad and versatile nuclear structure and reactions research program is centered at the LBL 88-Inch Cyclotron. The cyclotron is operated as a national facility and is equipped with two state-of-the-art ECR ion sources capable of producing high-charge-state ions of most elements.

The cyclotron is the site of the major initiative of the nuclear structure research community-Gammasphere-a new high-resolution gamma-ray detector array. Although still under construction, Gammasphere has been operating in an early-implementation mode with approximately 30 detectors and a resolving power greater than any operating array. It is being used by the LBL nuclear structure group and groups from around the world to study nuclear structure and exotic nuclear shapes, including superdeformed nuclei (distorted nuclei with a 2-to-1 ratio of major to minor axis). Already during the initial operation of the Gammasphere facility, these groups have discovered a large number of new superdeformed bands and have observed many new phenomena, including new nuclear symmetries, identical bands in different nuclei, and pairing correlations in superdeformed nuclei. An inner $4 \pi$ particle detector system, MicroBall, also enables studies of angularmomentum transfer and alignment in deep inelastic reactions with discrete gamma rays. Gammasphere is scheduled to begin its first phase of operation with 50 detectors in 1994. When completed in October 1995, Gammasphere will consist of an array of 110 large Compton-suppressed Ge gamma-ray detectors. Gammasphere is operated by LBL's Nuclear Science Division as a national user facility.

The nuclear structure of nuclei far from stability in the region of light proton-rich nuclei and of very heavy nuclei is also being investigated at the 88-Inch Cyclotron. Several factors, including the availability of intense (microampere) beams and the technology for handling radioactive beams, have combined to make the 88-Inch Cyclotron facility one of the leading laboratories in the world for the production and study of transuranic nuclei. Recent experiments at the cyclotron by the heavy element group confirmed the original discovery of element 106 (at LBL in 1974), enabling the discoverers to finally name the element-Seaborgium. This research program will continue to use the cyclotron to produce and characterize new elements and isotopes, to study nuclear reaction mechanisms, and to train students in modern nuclear and radiochemical techniques. LBL scientists are also participating in planning for the IsoSpin Laboratory, a proposed major new national initiative.

The Nuclear Science Division has joined with the Physics Division to establish an Institute for Nuclear and Particle Astrophysics, wherein researchers with common scientific goals that transcend divisional boundaries will work together to create a vital astrophysics community at LBL. Research topics include searches for right-handed currents and violations of time reversal invariance, studies of neutrino masses, double beta decay, neutrino oscillations, the solar neutrino problem, cosmic rays, and nuclear reactions of astrophysical importance. A major new project is the application of laser techniques for trapping atoms to study fundamental problems in nuclear physics. Recently, the first transport, cooling, trapping, and alignment of radioactive atoms using laser light was demonstrated in experiments 
at the 88-Inch Cyclotron. In collaboration with eleven other institutions from Canada, the U.S., and the United Kingdom, LBL is participating in the Sudbury Neutrino Observatory (SNO), an experiment to detect neutrinos from the sun and from supernovae. Installation of the detector components will begin in the underground observatory later this year.

The goal of the nuclear theory program at LBL is to address important problems at the frontier of nuclear science by developing the theory and methods necessary for the analysis and interpretation of experiments involving nuclei and for the prediction of as yet unobserved phenomena. In addition, the program aims at adding breadth to the overall nuclear research program.

The Isotopes Project of the Nuclear Science Division provides evaluated nuclear structure and decay data for the world nuclear physics community. The group has an expanding role in the coordination of the U.S. Nuclear Data Network and in the development of electronic access and publication of the extensive data files.

Planned future improvements at the 88-Inch Cyclotron include modernization of the radiation safety interlock system and an upgrade of the Advanced Electron Cyclotron Resonance (AECR) ion source so that it can operate at higher magnetic fields and frequency, further increasing the Cyclotron's performance for the heavy-ion beams $(A>100)$. A subsequent project will improve the flexibility and precision of the beam timing characteristics of the 88-Inch Cyclotron. The goal of this project is to develop a fast beam-chopping system that could chop out individual beam pulses and enable the study of short half-life nuclear states with lifetimes greater than $100 \mathrm{~ns}$ with the Gammasphere detector.

\section{High-Energy Physics Program Direction}

In high-energy physics, the Laboratory continues its strong and diverse program of experimental and theoretical research, including the development and operation of innovative detectors and research on advanced accelerator components and concepts. LBL is actively participating in the national effort to design future facilities, including an asymmetric $B$ Factory at PEP.

The Laboratory's experimental programs in high-energy physics focus on the properties of quarks and leptons, the basic constituents of matter. Their interactions are mediated through the gauge bosons, namely, massless photons and gluons and massive $\mathrm{W}$ and $\mathrm{Z}$ particles. Efforts at studying these particles emphasize the development of sophisticated detectors and their operation at colliding-beam facilities. Major experiments are in progress or in active preparation at SLAC and Fermilab.

LBL has a major role in both of the two large detectors at the Fermilab Tevatron Collider, the Collider Detector Facility and the D-Zero. Both detectors are now taking data. The effectiveness of the Collider Detector Facility has been enormously enhanced by the Silicon Vertex Detector, for which LBL was the lead institution. This work involved close collaboration among the Physics Division, the Engineering Division, and industrial suppliers. The Silicon Vertex Detector has achieved approximately $10-\mu \mathrm{m}$ resolution in the transverse plane. The result is that $B$ physics has been opened up to an unprecedented and unexpected extent. This detector has played a crucial role in the evidence for the t quark. LBL groups with this experiment are involved in analysis of $B$ decays, the $t$ quark search, and the 
measurement of the $\mathrm{W}$ mass. Meanwhile, the $\mathrm{D}$-Zero detector has started to produce physics remarkably quickly. LBL's contributions to the hardwarethe Vertex Detector and End Cap Electromagnetic Modules-are functioning well. LBL groups are involved in analysis of $W$ and $Z$ events. The future of $D$-Zero lies with upgrades, and LBL is heavily committed to the design and construction of a new silicon vertex detector, building on the experience at LBL gained in the development of this type of detector. The SVXII chip is being developed jointly with Fermilab.

In addition, technology-development efforts are directed toward ongoing detector construction and upgrade projects. For example, ultrahighresolution vertex detectors for MARK II at the Stanford Linear Collider and D-Zero at the Tevatron I were fabricated to provide spatial resolution on the order of tens of micrometers to detect the decays of very-short-lived particles. Another example is the development of ultrahigh-resolution solidstate detectors to search for neutrinoless double beta decay to measure a finite neutrino mass.

The Stanford Linear Collider has dramatically improved its performance. High luminosity running with good polarization is now regularly achieved. The LBL Compton Polarimeter is a key element of this program. A stringent test of the Standard Model is being made by measuring the left-right asymmetry. Continued running with improved polarization will give a result that will rival those of CERN's Large Electron-Positron collider ring results for precision on the weak mixing angle.

The Astrophysics Program of the Physics Division has three components: cosmic microwave background measurements, a search for dark matter, and a search for distant supernovas. The cosmic microwave background program may be extended with new satellite-borne detectors. The dark matter search has embarked on a several-year program of experimentation. The distant supernova search program has already discovered the several very distant supernova, including the most distant one ever observed. An expanded program should find a few dozen distant supernovas, enough to determine the mass density of the universe.

The Laboratory also has a strong theoretical particle physics group, whose work ranges from highly theoretical topics to others closely related to current experiments. A substantial effort is being devoted to theoretical studies in support of physics in the multi-TeV energy range.

The Particle Data Group performs a service to the world's high-energy physics community through its compilations of particle properties. Its recent strengthening includes making databases more accessible through computer links.

Advanced detector development is aimed at long-range research in detector problems relevant to proposed hadron colliders. The program emphasizes the development of radiation-hardened devices, pixel devices, monolithic amplifier arrays, and data-acquisition electronics.

Accelerator physics and engineering for the design and application of particle beams is an LBL core competency that has long played a leading role in research and development for high-energy physics facilities. In the recent past, for example, LBL experience with beam cooling resulted in successful systems at the Tevatron I. Most recently, LBL has turned its attention to high-luminosity lepton colliders such as B-meson factories and has been using its combination of analytical and practical expertise to solve a variety of problems such as beam-beam instabilities and damping of higher-order modes. 
A comprehensive, integrated approach to superconducting magnet $R \& D$ is a part of the competency that LBL applies to high-energy physics. The program emphasizes three major aspects of building higher-field and higherquality magnets. An ongoing effort to make wire for greater current density explores small filament diameters $(2-3 \mu \mathrm{m})$ and brittle superconductors such as niobium-tin. Improved cable designs are also a major focus of activity. These achievements come together in the development of stronger and more efficient accelerator-type magnets, one of which set a field-strength record of slightly more than 10 tesla in 1992 testing. Other highlights include efforts to make better magnets and to find better ways of designing magnets.

Other activities include accelerator design of the PEP-II for an energyasymmetric B Factory. Based on an LBL concept, the collider will use $\mathrm{e}^{+} \mathrm{e}^{-}$ collisions at different energies to produce $B$ mesons in a way that spreads out the collisions over space-time so that they are easier to study for evidence of CP-violating decays. The B Factory has now been approved for construction. Work will be carried out jointly by a team of engineers and scientists from SLAC, LBL, and LLNL. It is anticipated that LBL will be responsible for about $\$ 25$ million of construction activities, focusing mainly on magnets and vacuum systems for the newly constructed low-energy ring.

In a futuristic program aimed at a next-generation, high-energy electron collider, LBL scientists, collaborating with LLNL and SLAC colleagues, are working on the Two-Beam Accelerator. This concept, pioneered at LBL, uses either a free-electron laser or a relativistic klystron to generate extreme levels of microwave power, which is then applied to a high-gradient linac structure.

LBL is conducting radiation health and instrumentation-development projects for the National Aeronautics and Space Administration (NASA). In collaboration with Colorado State University, Fort Collins, LBL is recognized as the NASA Specialized Center for Research and Training (NSCORT) in these areas. LBL also collaborates with various NASA groups to investigate the cosmic microwave background radiation and to develop gamma and $x-$ ray detector systems for various space applications, such as for the WIND Spacecraft and Nuclear Astrophysics Explorer. LBL scientists are developing a superconducting magnetic spectrometer for the space station to measure cosmic-ray particles and gamma rays in search of exotic matter. Other ongoing research concerns nitrogen recycling in a closed ecological lifesupport system for long-term space missions.

\section{Health and Environmental Research Program Directions}

Life-sciences-related research activities include six research program areas: gene expression and genome mapping; structural biology; nuclear medicine and functional imaging; carcinogenesis, mutagenesis, and radiation biology; environmental and health-effects research; and measurement technology. These programs form a core of research conducted for DOE's national programs supported by the Office of Health and Environmental Research (OHER). Program expansions are anticipated in human genome research, structural biology, gene expression, growth regulation, molecular medicine, and environmental science.

\section{Gene Expression and Genome Mapping}

Important research growth areas for $L B L$ are studies on human genome structure and regulation of gene expression. Research at the Human Ge- 
nome Center includes generation of physical and genetic maps, identification and localization of expressed genes on human chromosome 21, and a major effort for the development of techniques and automation for efficient sequencing of human DNA. Analysis of the biologically relevant signals culled from sequence information is under way. A physical map of the Drosophila genome, along with the identification of expressed genes, is being developed in collaboration with UC Berkeley and Harvard University. The biological function of the human DNA sequences identified by the Human Genome Center will be determined using genetically engineered mice developed by researchers at LBL.

LBL's Life Sciences Division conducts several related research programs on gene expression within mammary-gland and blood-forming systems. The highly secretory mammary epithelial cells provide excellent models for gene expression and chemical- and radiation-induced carcinogenesis and are now also providing vehicles for production of genetically engineered foreign genes. LBL has identified hematopoietic research for expansion. Bloodforming cells are important targets of radiation-induced damage and are versatile models of stem-cell differentiation and regulation of gene expression.

Molecular cytogenetics describes a set of pioneering diagnostic methodologies for biomedical applications-many of which have already made a significant contribution to the megabase sequencing effort in the Human Genome Project. The research program centers in particular on refinements in hybridization technology to increase the speed, sensitivity, and specificity of hybridization to allow rapid identification and mapping of genetic aberrations.

\section{Structural Biology}

One thrust of LBL's structural biology program is directed toward x-raybased research at the ALS (see the Structural Biology Initiative, p. 4-6), although the program has other facets as well. X-ray crystallography, electron crystallography, and NMR spectroscopy focus on protein and nucleic acid structures. Several studies are aimed at determining how proteins regulate the expression of genes by examining the structure of the protein-nucleic acid complexes. These latter studies will use advanced techniques such as $\mathrm{x}$-ray diffraction made possible by the ALS.

Studies based on high-resolution electron crystallographic structure analysis and $x$-ray diffraction are also elucidating the structure of specific membrane proteins involved in transmembrane signaling and ion channels. Using unique techniques for electron diffraction and imaging of crystalline sheets of membrane proteins, structural studies will focus on such membrane-bound proteins and integral membrane proteins as growth-factor receptors, chemotaxis receptors, and receptors for extracellular matrices. LBL's new intermediate-voltage electron microscope, which allows viewing of much thicker specimens than can be examined with conventional electron microscopes, is enabling researchers to study complex structures, including that of the isolated mitotic spindle, which can be prepared as a frozen, hydrated specimen. Related work will exploit circular-dichroism microscopy, electron microscopy, and novel microscopic-imaging capabilities from the ultraviolet and soft $x$-ray beams of the ALS.

Hard $x$-ray radiation from a wiggler will make it possible to develop technical capabilities in synchrotron-based protein crystallography that have 
never been attempted before. One such advance is specialized $x$-ray optical devices that will provide multiplexing and time-sharing capabilities leading to order-of-magnitude more efficient use of the available beams. Another is $\mathrm{x}$-ray microfocus technology to extend data-collection capabilities to protein microcrystals as small as $20 \mu \mathrm{m}$ or less.

Current research is exploring new concepts in the realm of soft x-ray microscopy of biological specimens. The goal is to test ideas such as highresolution fluorescence microscopy (using lanthanide chelate labels) and the mapping of elemental distribution within subcellular compartments. A scanning $\mathrm{x}$-ray microscope designed to exploit these techniques would use a specially designed undulator, optimized for light output in the "water window" range of $\mathrm{x}$-ray wavelengths.

LBL has also established a unique center of excellence, the Program for Biomolecular Design, that will bridge chemical, biological, and computer sciences by combining the expertise at LBL and UC Berkeley. The program will catalyze an understanding of biological systems with the ability to analyze and manipulate chemical structure. The aim is to redesign the natural biological molecules to create new classes of novel biomolecular structures with applications to major problems in the medical, biological, and environmental sciences relevant to DOE missions.

\section{Nuclear Medicine and Functional Imaging}

Research in nuclear medicine will include new studies in molecular biology and continuing studies of improved radiopharmaceuticals and advanced instrumentation for applications to medical science. A systematic search for new, ultrafast heavy-atom scintillators will continue, as well as the development of solid-state photodetectors for multilayer, high-resolution, positron-emission tomography. A new multilayer, high-resolution tomograph is planned for use in medical studies of the human and animal brain and heart.

Methods for the production of radioisotopes and for the labeling of biochemical substrates to be used in noninvasive imaging have contributed to the effective use of these diagnostic-imaging tools. Newly developed radioisotope generators give greater flexibility to the application of shortlived, positron-emitting isotopes by using long-lived parent radioisotopes, and automated elution techniques for the delivery of short-lived radionuclides.

Based on LBL's tradition of radiobiology and isotope chemistry, the Laboratory is supporting DOE's Health and Environmental Research program to conduct isotope-based research and to provide tracer isotope resources for the nation's biomedical research community. LBL is a consultant in studies for a national biomedical tracer facility, the purpose of which is to maintain a U.S. research infrastructure for stable and radioactive tracers that are vital in biomedical research, including metabolic studies, diagnostic imaging, and other applications.

In parallel with an emerging national trend in molecular nuclear medicine, studies in four new areas have been initiated: (1) use of modern instrumentation and dosimetry, along with human genetic studies and transgenic animal models, to pursue the relationship among variations in the genome, low-density lipoprotein receptors, and the occurrence of atherosclerosis; (2) use of advanced noninvasive methods of nuclear medicine and NMR to study the relationships among ion-channel protein aberrations, 
brain physiology associated with mental disorders, and the genome; (3) development of labeled DNA probes of specific proteins associated with inflammatory diseases, autoimmune conditions, atherosclerosis, and cancer; and (4) use of nuclear medicine methods to monitor gene therapy.

\section{Carcinogenesis, Mutagenesis, and Radiation Biology}

LBL has a number of strong research programs in carcinogenic mutagenesis and radiation biology:

Control of Growth, Differentiation, and Genomic Stability. LBL has strong programs in the elucidation of mechanisms that control cell proliferation, differentiation, and genomic stability, and the abnormalities that occur in each of these processes during tumorigenesis.

Hormones and Extracellular Matrix. Research is directed toward identifying the intracellular molecules that mediate cells' responses to hormones, particularly the sex steroids, and the extracellular matrix (ECM). Investigators have demonstrated the importance of hormones and ECM in maintaining the balance between growth, differentiation, and death in normal tissue; mediating the tissue response to radiation; and initiating neoplasia. Researchers are now working towards understanding the molecular bases for the actions of hormones and ECM and developing methods to modulate their activities.

Hematopoiesis. Using rodent and human cells in culture and in vivo, investigators are examining how differentiation and malignant transformation are controlled in blood-forming cells. In particular, there is a substantial effort to understand how tissue-specific gene expression is regulated, to identify chromosomal changes that occur in these cells after exposure to carcinogenic stimuli, and to characterize and isolate hematopoietic stem cells.

Mammary Biology. A large, multi-investigator program in mammary gland biology aims to understand the extracellular signals and intracellular mediators that control mammary epithelial cell growth, development, functional differentiation, and death, particularly as it relates to various stages of tumorigenesis. Investigators are studying epithelialstromal interactions, ECM-cell and cell-cell interactions, growth factor and ECM receptors and the intracellular signaling pathways, and transcription factors that are key regulators of growth and differentiation.

Oncogenes and Tumor Suppressor Genes. In another area of research, investigators are learning how protooncogenes (which, through mutation, become oncogenes) and tumor suppressor genes function in normal and neoplastic cells. Using both viral and cellular genes, investigators are determining the role of protooncogenes and tumor suppressor genes in the cell phenotype resulting from environmental signals, such as growth factors and ECM and DNA damaging agents, as well as genetic programs, such as replicative senescence and terminal differentiation.

Radiation and Chemical Carcinogenesis. LBL researchers are studying the biological responses of cells and organisms to environmental hazards such as ionizing radiation. These studies will enable an understanding of the consequences of low-dose exposure, which is important in assessing the risk associated with space exploration, certain occupational hazards, and medical diagnostic techniques. These 
studies also are providing a molecular understanding of how DNA lesions are acquired and repaired.

DNA Repair. Activities in the area of DNA repair include the cloning of human genes by complementation of repair defects in lower organisms, studies on how the structural constraints and genomic organization affects damage and repair, and the inducible responses to DNA damage.

Excellence in measurement technology is key to the success of OHER programs. Refinements in sensors and analytical techniques have been developed at LBL for a number of years. New detector configuration and the discovery of new scintillation will significantly benefit the positronemission tomography program of OHER. Research and development of the associated electronic signal-processing techniques complement this work. OHER support for $x$-ray detectors at the ALS and other synchrotron sources will utilize LBL expertise and provide significant advances in image resolution and chemical characterization of biological materials.

\section{Work For Others-National Institutes of Health}

The success of the DOE biosciences and environmental sciences programs at LBL has depended not only on DOE support but also on complementary NIH-supported research that is closely coupled to these programs. Several critical technologies recently identified as being of high priority for advancement by $\mathrm{NIH}-$ specifically molecular medicine, biotechnology, and structural biology - are all major components of the life sciences program at LBL. These technologies build on the unique facilities and expertise available at LBL and point toward a growing interaction of DOEand NIH-funded research in pursuing new goals in biology and medicine.

NIH supports programs on radionuclides, NMR, diagnostic image reconstruction, and radiopharmaceuticals related to advanced instrumentation and disease treatment. Other major $\mathrm{NIH}$-funded programs involve lipoproteins and their relationship to cardiovascular disease, biological structure analysis by electron crystallography to characterize cell-membrane proteins and viruses, and the intracellular molecular structure of DNA and sickle hemoglobin.

$\mathrm{NIH}$ applies LBL's unique resources to investigations of the human genome and in carcinogenesis and mutagenesis. Repair and recombination in yeast and the genetic effect of carcinogens will continue to be major foci. Cell nuclei are studied by circular dichroism and related techniques. The Laboratory's capability in culturing human mammary epithelial cells is used to study breast cancer.

The National Tritium Labeling Facility conducts research into the labeling of compounds with tritium. LBL also conducts a program on intermediate-voltage biological electron microscopy under NIH sponsorship. NIH also supports research on oxygen radicals and aging, environmental tobacco smoke, soil transport of gas pollutants, and ecotoxicology assays.

In the life sciences, research on human lipoprotein function and genetics is supported by the National Dairy Board through Children's Hospital in Oakland. The UC California Tobacco Research Institute supports research on carcinogenesis. The National Cancer Institute supports basic research on how carcinogenesis is initiated by chemicals and radiation. Additional 
studies are funded to investigate how normal growth and cancer cells are controlled by their microenvironments.

\section{Laboratory-Directed Research and Development Program Direction}

This program contributes to scientific staff capability and vitality through the support of new research programs of merit and potential. Examples of project areas eligible for support include:

- Work in forefront areas of science and technology that enrich Laboratory research and development capabilities

- Advanced study of new hypotheses, new experiments, and innovative approaches to development of new concepts or knowledge

- Experiments directed toward proof of principle for initial hypothesis testing or verification

- New-device studies to explore possible application to instrumentation or experimental facilities

Recent achievements sponsored by the Laboratory-Directed Research and Development (LDRD) program in the energy sciences include research in interface and nanostructure materials, blue-light-emitting diodes and lasers, novel displacement detectors for seismology, time-of-flight spectroscopy of rare gases, $x$-ray reduction lithography, novel chemical dynamics studies, studies of fullerenes, new high-pressure semiconductor processing, and advanced techniques for electron microscopy. Research achievements in the general sciences were directed towards prototype tracking and timing detectors, ultraviolet free-electron lasers, new types of silicon-tracking systems, and new data-acquisition systems. Achievements in the biosciences area included analysis of a cell-cycle regulatory gene, single DNA molecule chemistry, testing of novel mismatch repair enzymes, $x$-ray crystallographic studies of ribonucleic acid (RNA), and environmental air pollutants and oxidative stress. Achievements in the resources and operations areas included studies on neutron detectors for future applications and studies in high-density packaging of high-frequency integrated circuits. The Annual Report on the LBL Laboratory-Directed Research and Development Program is available from the Office for Planning and Communications. Planning documents that indicate program directions and projected resources are prepared annually.

Laboratory-Directed Research and Development Program ${ }^{a}$

\begin{tabular}{lcccc}
\hline Category & $\begin{array}{c}\text { FY 1993 } \\
\text { Actual }\end{array}$ & $\begin{array}{c}\text { FY 1994 } \\
\text { Authorized }\end{array}$ & $\begin{array}{c}\text { FY 1995 } \\
\text { Authorized }\end{array}$ & $\begin{array}{c}\text { FY 1996 } \\
\text { Projected }\end{array}$ \\
\hline Funding (\$M) & 5.1 & 8.5 & 7.6 & 7.9 \\
Projects Approved & 48 & 47 & - & - \\
\hline
\end{tabular}

apreliminary estimate of LBL actual year Budget Authority (B\&R code EM).

\section{University and Science Education Program Direction}

The Center for Science and Engineering Education (CSEE) develops and implements programs that utilize the resources of Lawrence Berkeley 
Laboratory (LBL) to improve the quality of mathematics, science, and technology education in the United States. On an annual basis, CSEE educational activities reach thousands of students and faculty from kindergarten through postgraduate school. With a total annual budget of $\$ 2.8$ million, CSEE programs are funded primarily by the Department of Energy, with additional funding from the National Science Foundation and the National Institutes of Health.

\section{Ongoing Initiatives}

Since its inception in 1987, CSEE has developed programs in four areas. By virtue of their history, programmatic excellence, and national reputation, these programs can be characterized as the Center's ongoing initiatives.

Precollege Programs encompass eight subprograms that focus on systematic reform and student and faculty development. Especially noteworthy is the Bay Area Science and Education Collaboration (BASTEC), which provides a broad range of educational activities and curriculum support for the Oakland Unified School District, one of the country's largest urban school districts. In addition to BASTEC, the Precollege Program sponsors partnerships and institutes to enrich student and faculty development in math and science.

College and University Programs bring students to LBL to work closely with scientists in summer and semester research programs. The programs are characterized by long-term, close mentor relationships that provide participants with in-depth exposure to the world of scientific research.

Educational Outreach Programs focus on supporting the educational needs of students and teachers in the San Francisco Bay Area. The program is noted for its cadre of LBL employees who volunteer to work in individual classrooms and for summer research programs for high school students from groups that are underrepresented in the sciences.

The Science Consortium is a collaboration of three entities-LBL, Jackson State University in Mississippi, and the Ana G. Méndez University System in Puerto Rico. These three organizations, working together, promote computer and science education in the academic institutions through collaboration with LBL scientists.

\section{New Initiatives}

To respond to changes and demands in education, the CSEE has two programs in a developmental phase.

The Educational Technology Program coordinates the educational efforts of various research divisions at $L B L$ to promote and deliver materials for the nation's classrooms through the World Wide Web. For example, the Physics Division has developed the groundbreaking program Hands-On Universe that links university astrophysics research with high school classrooms and museums. Through the use of computers, students gain access to professional grade telescopes, current analytical tools, and the training to use them. 
The Community College Connection provides educational services to students and faculty in community college career education programs. Program components include a Cooperative Work Education Program, a Speakers' Bureau, LBL tours designed to augment course curricula, and a summer faculty research associate program. Future plans include a school-to-work component for community college vocational students. 


\begin{tabular}{|c|c|c|c|c|c|c|}
\hline \multirow[b]{2}{*}{ Program } & \multicolumn{3}{|c|}{ FY 1993} & \multicolumn{3}{|c|}{ FY 1994} \\
\hline & Totals & Minorities $^{\mathrm{a}}$ & Women & Totals & Minorities $^{\mathrm{a}}$ & Women \\
\hline \multicolumn{7}{|l|}{ Precollege Programs } \\
\hline $\begin{array}{l}\text { Bay Area Science and Technology } \\
\text { Education Collaboration (Teachers) }\end{array}$ & 908 & 411 & 621 & 902 & 450 & 675 \\
\hline $\begin{array}{l}\text { Bay Area Science and Technology } \\
\text { Education Collaboration (Students) }\end{array}$ & 4,215 & 3,837 & 2,078 & 4,672 & 3,928 & 2,216 \\
\hline $\begin{array}{l}\text { Teacher Research Associates } \\
\text { (Teachers) }\end{array}$ & 44 & 7 & 21 & 40 & 9 & 22 \\
\hline $\begin{array}{l}\text { High School Honors Program } \\
\text { Life Sciences (Students) }\end{array}$ & 64 & 12 & 35 & 64 & 8 & 36 \\
\hline $\begin{array}{l}\text { High School Science } \\
\text { Symposium (Students) }\end{array}$ & 97 & 27 & 58 & - & - & - \\
\hline $\begin{array}{l}\text { Updating Science Knowledge } \\
\text { for Instruction (Teachers) }\end{array}$ & 450 & 75 & 208 & 420 & 95 & 257 \\
\hline Science Bowl (Students) & 80 & 10 & 12 & 96 & 10 & 15 \\
\hline $\begin{array}{l}\text { Student Research Assistantships } \\
\text { (Division Hires) }\end{array}$ & 200 & 50 & 90 & 190 & 45 & 85 \\
\hline Education Outreach ${ }^{b}$ & 1,500 & 7,000 & 8,000 & 20,000 & 8,000 & 10,000 \\
\hline Student Research Program & 15 & 15 & 9 & 25 & 25 & 19 \\
\hline $\begin{array}{l}\text { National Teacher Enhancement } \\
\text { Program (NTEP) }\end{array}$ & - & - & - & 19 & 10 & 13 \\
\hline Summer Youth Employment Training Program & 3 & 3 & 2 & 15 & 15 & 7 \\
\hline Oakland Science Explorers Program & 1,200 & 700 & 600 & 1,200 & 700 & 600 \\
\hline \multicolumn{7}{|l|}{ Undergraduate Programs } \\
\hline Alliance for Minority Participation & - & - & - & 4 & 4 & 0 \\
\hline Bridge Program & - & 一 & - & 5 & 0 & 0 \\
\hline $\begin{array}{l}\text { Community College Connection } \\
\text { (Faculty Development) }\end{array}$ & - & - & - & 236 & 43 & 84 \\
\hline Community College Transferc & 3 & 3 & 0 & 5 & 4 & 2 \\
\hline Laboratory Co-op Programc & 7 & 3 & 2 & 18 & 2 & 6 \\
\hline $\begin{array}{l}\text { Environmental Management Career } \\
\text { Opportunities for Minorities }{ }^{\mathrm{c}}\end{array}$ & 3 & 3 & 3 & 1 & 1 & 1 \\
\hline $\begin{array}{l}\text { Minority Access to Energy } \\
\text { Research Careers }\end{array}$ & 9 & 8 & 3 & 8 & 8 & 2 \\
\hline LBL/JSU/AGMUS Science Consortium & 23 & 22 & 12 & 19 & 19 & 17 \\
\hline $\begin{array}{l}\text { Science and Engineering } \\
\text { Research Semester }\end{array}$ & 28 & 2 & 10 & 24 & 3 & 9 \\
\hline Faculty/Student Teams (Faculty) ${ }^{c}$ & 2 & 2 & 1 & 5 & 3 & 1 \\
\hline
\end{tabular}

aMinority numbers are for underserved groups only.

bThis program covers Northern California.

${ }^{\mathrm{C} E s t i m a t e}$ includes summer programs. 
$L B L$ 's proposed Elise accelerator will perform crucial tests to demonstrate the feasibility of a full-scale driver for inertial confinement fusion. The accelerator will perform experiments on bending, drift compression, and focusing. Acceleration modules and a four-beam injector will be added to demonstrate all features of the fusion driver.

\section{$"$ ": ENERGY RESOURCES}

Energy resources efforts at $L B L$ are in line with DOE's strategy to encourage efficiency; advance alternative and renewable energy technologies; increase energy choices for all consumers; ensure adequate supplies of clean, conventional energy; and reduce U.S. vulnerability to external events. LBL efforts support DOE's key program areas of energy efficiency and renewable energy, fossil energy, fusion energy, civilian radioactive waste management (discussed under environmental quality), and least-cost energy supply-and-demand management systems. The following sections identify key LBL initiatives in support of these strategies and describe research and development directions. LBL's energy resources initiatives include the following:

- Heavy-Ion Fusion Driver

- Energy Efficiency for the Climate Change Action Plan

- Advanced Petroleum Technologies Alliance

\section{Heavy-Ion Fusion Driver Initiative}

The U.S. Heavy-Ion Fusion Accelerator Research (HIFAR) Program is building a research database to assess and develop heavy-ion accelerators as drivers for an inertial-fusion energy source for commercial power generation. The results of the successful Single-Beam Transport Experiment and the Multiple-Beam Experiment provide encouragement and justification to conduct larger, more complex experiments. The features of a full-scale fusion driver, yet to be demonstrated, include the components for beam merging with minimal phase-space density dilution, transition from electrostatic to magnetic focusing, transport using bending and focusing magnets, drift compression, and focusing onto a small target. To investigate these important accelerator components and beam manipulations, LBL proposes to build a new accelerator known as Elise, an induction linac system.

The Elise Program has three parts: (1) the Elise accelerator construction project; (2) construction of additional acceleration modules and a four-beam injector; and (3) a series of experiments to be performed with the accelerator, modules, and injector. Initially, Elise will use an existing one-beam, 2$\mathrm{MeV}$ injector and will accelerate $\mathrm{K}^{+}$ions to $5 \mathrm{MeV}$. The $5 \mathrm{MeV}$ beam will

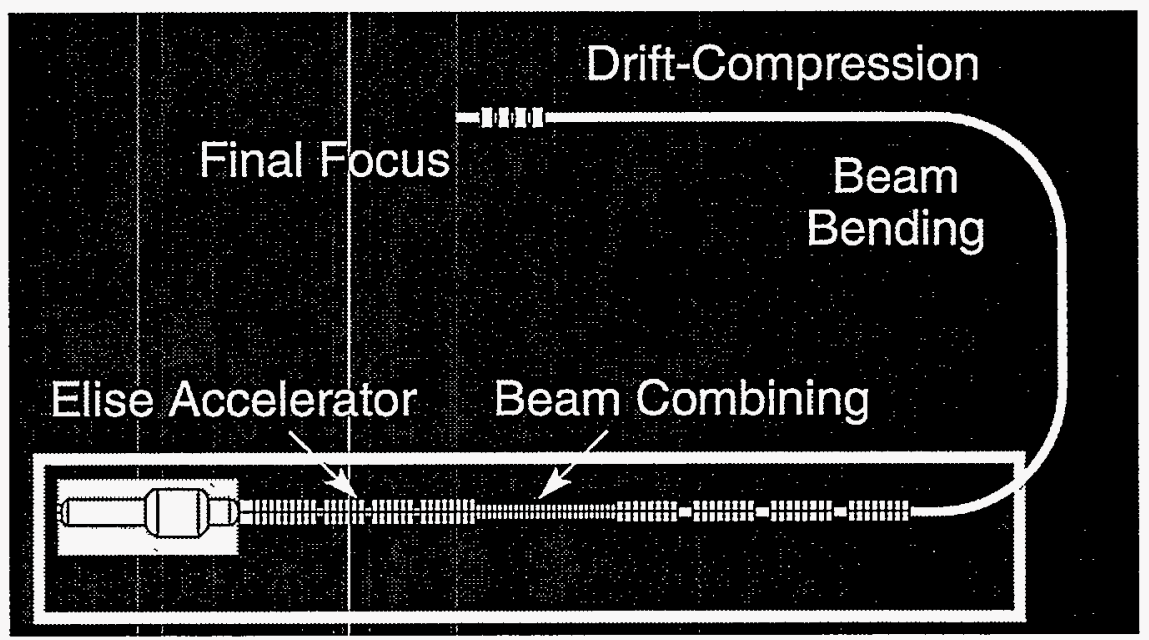


be suitable for a wide variety of experiments on bending, drift compression, and focusing; however, additional acceleration modules and a four-beam injector will be required to demonstrate all the features of the fusion driver described above. To minimize cost, the Elise accelerator is shorter and has fewer beams than a full-scale driver, but the beams will have the same diameter and line charge density as driver beams. Elise will therefore test much of the important driver physics at full scale. The Elise project also provides a pathway for the development of pulsed-power technology applicable to the production of pulsed neutrons. Incremental costs of Elise hardware above the base HIFAR Program are indicated in the following table, which assumes a construction project start in FY 1996. Additionally, the heavy-ion fusion base program will continue theoretical studies and small experiments required to investigate fundamental aspects of heavy-ion fusion physics. It also will address the design of the Elise experimental program that will begin in FY 2000 and require a support level of $\$ 8$ million/year. These costs include all required protective systems for ES\&H hazards, which are similar to those of the existing HIFAR program. A Conceptual Design Report addresses ES\&H requirements and NEPA/CEQA documentation.

Heavy-Ion Fusion Driver Initiative Resource Requirements $(\$ M)^{\mathrm{a}}$

\begin{tabular}{lccccccc}
\hline Category & 1995 & 1996 & 1997 & 1998 & 1999 & 2000 & Total \\
\hline Operating & 2.5 & 2.5 & 2.5 & 2.5 & 2.5 & 2.5 & 15.0 \\
Construction & 3.1 & 3.6 & 4.8 & 4.8 & 4.8 & 4.5 & $25.6^{\mathrm{b}}$ \\
\hline
\end{tabular}

aPreliminary estimate of LBL Budget Authority (B\&R code AT). Construction costs include $8.61 \% \mathrm{LBL}$ overhead.

bIncludes R\&D associated with construction, and preops. Some of this budget will be contracted to other labs or industry.

\section{Energy Efficiency for the Climate Change Action Plan Initiative}

LBL is proposing to assist DOE in its effort to implement actions to reduce greenhouse gas emissions as called for in the Climate Change Action Plan (CCAP). This initiative consists of three distinct elements, each of which is important to the nation if the CCAP is to be successful:

Assistance with specific elements in the CCAP. Among the fifty or more elements in the Plan, LBL has unique expertise in four or five (e.g., the "Cool Communities" initiative, "Rebuilding America," appliance standards, energy-efficient mortgages) and a great deal of expertise in another ten or more (e.g., the market pull/market push strategies for residential and commercial energy efficiency, energy ratings for buildings). We will establish a strong technical program that is directly supportive of these elements within the CCAP.

Monitoring and evaluation. There is an enormous need for monitoring and evaluation of the programs that are established for each of the elements in the CCAP. LBL will develop and extend the state of the art in monitoring of buildings (in terms of the collection of more accurate and meaningful data on the energy, environmental, and physical/ productivity-enhancing performance of buildings and in reducing the cost of such data collection). This capability will then be applied to 
$L B L$ 's expertise in a number of energy and environment research areas has led not only to concrete savings to the nation, but also has led to the development of a broad capability to support analysis of environmental and economic impacts of policy decisions. This table of Annual Energy Savings (1993) indicates four specific areas of $L B L$ 's research capabilities.

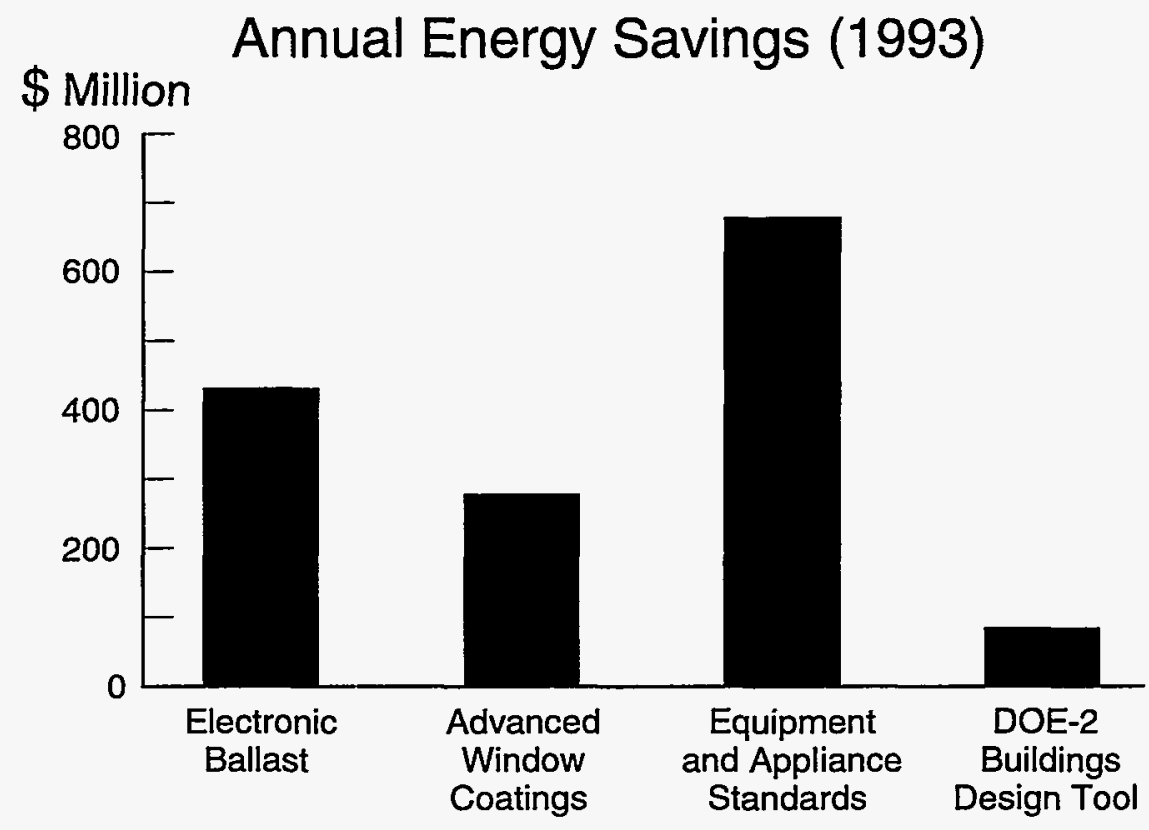

data gathering and evaluating the performance of key CCAP programs (and the specific actions that are part of the programs).

Research base. A significant research effort-particularly relating to new technology to improve the energy, environmental, and physical performance of buildings-is essential if the elements of the CCAP are to have major and increasing impact after the year 2000. LBL will carry out research on window and lighting systems, a new generation of energy-efficient appliances, advanced techniques for building design and for ensuring that the buildings are constructed and perform as intended, and advanced sensors and systems for more cost-effective monitoring. These advances will be made in conjunction with the development of the national information infrastructure (the "Information Superhighway") for such activities as information transfer, remote monitoring of building performance, and remote access (e.g., by industry) to LBL test facilities, databases, and models.

In parallel with this initiative, $L B L$ is preparing a proposal for a new facility that would enhance the programmatic activities by having uniquely focused laboratories, "showcasing" energy-efficient technologies and providing unique access for users from industry.

Energy Efficiency for the Climate Change Action Plan Initiative Resource Requirements $(\$ M)^{\text {a }}$

\begin{tabular}{lccccccc}
\hline Category & 1995 & 1996 & 1997 & 1998 & 1999 & 2000 & Total \\
\hline Operating & 2.0 & 3.0 & 5.0 & 5.0 & 5.0 & 5.0 & 25.0 \\
Construction & 0.0 & 0.0 & 0.0 & 0.0 & 0.0 & 0.0 & $0.0^{\mathrm{b}}$ \\
\hline
\end{tabular}

aPreliminary estimate of LBL actual year Budget Authority (B\&R code EE).

bProposal for new facility under preparation. 


\section{Advanced Petroleum Technologies Alliance Initiative}

The Earth Science Division has taken the lead to involve LBL in a number of new initiatives that address technology areas specific to the domestic oil and gas industry, an industry hit hard by declining oil prices and the high costs of finding new oil and producing oil tightly bound in mature fields. In FY 1994, LBL became a member of the Natural Gas and Oil Technology Partnership, joining SNL, Los Alamos National Laboatory (LANL), and LLNL in a DOE Laboratory partnership sponsored by DOE's Fossil Energy (FE) Program to make laboratory capabilities available to the oil and gas industry. Through the partnership, LBL and its industry collaborators competed for DOE/FE funds in the program area called the Oil Recovery Technology Program. LBL received $\$ 810,000$ to start four new projects directed toward improving heavy-oil recovery from California's diatomite reservoirs. These projects were developed as a result of LBL's efforts to build a California oil and gas alliance with 12 California-based oilproducing companies.

Another petroleum initiative involving LBL participation is the FY 1995 Presidential Initiative, called the Advanced Computational Technology Initiative (ACTI). Subject to Congressional approval, ACTI asks for a redirection of $\$ 50$ million in program funds to address petroleum industry-driven collaborative projects with the Laboratories on topics related to lowering the costs and risks of oil/gas exploration and production. LBL will compete for the approximately $\$ 30$ million of non-DP monies, and we expect to submit proposals totaling $\$ 2$ million for FY 1995 funds, with slightly larger requests to follow in FY 1996 and beyond.

These initiatives are driven by the effect of the decline in oil prices on the economic viability of domestic oil production. The Secretary of Energy has established a four-point energy initiative designed to decrease dependence on oil imports, increase gas supply and demand, reduce exploration and production cost, and reconcile environmental and energy differences. The California producers have been especially hard hit because of stringent and duplicative regulations and the fact that much of California's oil is relatively expensive to extract. Further, California's production problems are relatively unique and, although California is the fourth-largest oil-producing state, DOE's previous programs have largely ignored these special problems. Beyond California, companies throughout the country will live or die depending on how economically they can solve a variety of production and environmental problems.

LBL has special expertise that can contribute directly to solving technical problems in the petroleum industry. Many of the problems faced by industry can be addressed through better characterization of the underground environment. LBL's Earth Science Division is a center of excellence for advanced characterization technology, including geophysical imaging and fluid-flow modeling of heterogeneous and fractured rocks. We are also very strong at developing approaches to in situ engineering to control or effect a variety of processes that occur underground. We have developed technology to analyze and control complex flow processes under multiphase, multicomponent, nonisothermal, and reactive conditions, all of which occur in oil and gas reservoirs. Unique technology to create underground barriers through the production of foam or advanced materials has been developed here. Many of the industry's problems have resulted from traditional separation between disciplinary approaches to problems. We 
have developed an integrative style of research at the Earth Science Division, which by itself contributes to important advances. Specific research proposals will be reviewed for NEPA and CEQA compliance.

Advanced Petroleum Technologies Alliance Initiative Resource Requirements $(\$ M)^{a}$

\begin{tabular}{lccccccr}
\hline Category & 1995 & 1996 & 1997 & 1998 & 1999 & 2000 & Total \\
\hline Operating & 3.0 & 3.2 & 3.5 & 4.0 & 4.0 & 4.0 & 21.7 \\
Construction & 0.0 & 0.0 & 0.0 & 0.0 & 0.0 & 0.0 & 0.0 \\
\hline
\end{tabular}

aPreliminary estimate of $L B L$ actual year Budget Authority (B\&R code $A C$ ).

\section{Energy Efficiency and Renewables Program Direction}

The LBL program in Energy Efficiency and Renewable Energy comprises a broad set of related activities that provide research support and technology development in the furtherance of national goals to reduce energy demand and cost to consumers, balance environmental concerns with economic development, and enhance energy security. LBL's programs are principally in electrical-energy storage and distribution, buildings, industry, transportation, utility systems, and geothermal systems.

\section{Utility Technologies}

LBL's programs provide assistance to utility planning and renewable energy supply and research that will yield long-term options for utilities. An expanding effort in Integrated Resource Planning is directed toward providing information and analytic methods for incorporating conservation and renewable options in utility planning on an equivalent basis to conventional supply options. This effort is carried out in close collaboration with the National Association of Regulated Utility Commissioners.

The work on electrochemical energy storage, described below under Transportation Technologies, will provide stationary energy-storage options for load-leveling applications. In another effort, work is ongoing to understand the effects of electromagnetic fields on biological systems.

A multidisciplinary program addresses the characterization and development of geothermal energy resources. The current program consists of field, laboratory, and theoretical studies covering four principal technical areas: delineation and evaluation of geothermal systems, definition of reservoir processes, modeling of reservoir dynamics and exploitation effects, and optimization of field-management practices.

Reservoir technology work will lead to more accurate predictions of the responses of a geothermal reservoir to exploitation for optimum management through carefully designed fluid-production and injection operations. Joint field projects with U.S. geothermal developers and utilities continue to be highly productive, as do collaborations with organizations in Mexico, Iceland, and Italy.

Currently, LBL is investigating The Geysers, the largest geothermal field in the world, as well as several other systems in California and Nevada. This investigation is part of a DOE-industry multi-institutional collaboration. More generally, the U.S. geothermal industry, including utilities, continues to be assisted by LBL's geothermal program in its effort to develop hydro- 
thermal (hot water and steam) resources in the U.S. and abroad. Instrumentation, field techniques, data interpretation methods, and computer codes developed at LBL, which are used in locating, evaluating and optimizing the exploitation of geothermal systems, are being transferred to the private sector. The assistance to industry also includes the collection and release of information on geothermal prospects throughout the world.

LBL is performing extensive analysis of energy demand in China: historical studies of determinants of energy intensity, case studies of energyefficiency decision making in industrial facilities, analysis of possible energy-efficiency investments, and assessments of energy-efficiency business opportunities in China.

Magnetic-field interactions are being evaluated in experimental animal systems and in tissue and cellular systems potentially sensitive to this nonionizing radiation. This program will develop theoretical models of magnetic-field interactions with biological systems and provide essential data for assessing the potential effects of magnetic fields.

\section{Industry Technologies}

This effort focuses on advanced industrial concepts, including energyefficient chemical separations, the opacification of aerogels for highperformance insulation in non-view applications (e.g., refrigeration), and other industrial applications. Work is under way in two projects on catalysts for industry: theory-assisted design of metal and zeolite catalysts; and chemistry, immunology, and modeling as tools for the rational design of stable, active enzymes.

\section{Transportation Technologies}

LBL manages the Exploratory Technology Research Program, which is the electrochemical research arm of DOE's Office of Propulsion Systems. The primary goal of this program is to advance the development of highperformance rechargeable batteries and fuel cells for use in electric vehicles. The battery performance goals for this program have been established by the U.S. Advanced Battery Consortium. LBL research areas include exploratory $R \& D$ on new electrochemical systems, supporting research for advanced rechargeable batteries currently under development, materials science research for improved battery components, fundamental electrochemical research to improve electrochemical energy-conversion efficiency, and characterization of advanced electrodes and electrolytes for use in fuel cells.

Based on its work in building technologies, LBL is developing switchable glazings for automobiles and other vehicles. Optical control to reduce solar heat gain will permit down-sized air conditioners and thus reduce use of chlorofluorocarbon (CFC) refrigerants as well as increase mileage.

LBL is working in conjunction with the other DOE multiprogram Laboratories to assist DOE in its role in the federal-industry Partnership for a New Generation of Vehicles (PNGV). LBL expertise that is available to the PNGV includes the above areas as well as combustion and emissions, lightweight materials, and improved manufacturing techniques.

\section{Building Technologies}

LBL will continue activities related to residential and commercial buildings in a program of laboratory and field research, modeling, data analysis, and partnerships with industry to accelerate market impact of our 
research. This work is a coordinated systems approach to designing building components and whole buildings with improved energy efficiency and better conditions for human health, comfort, and productivity. Modeling and field measurements verify results on economic costs and benefits of using energy efficiently. Important aspects of the work include measurements of indoor air quality and possible health effects of proposed efficiency measures.

The Laboratory has a lead role in applied research in four areas related to energy efficiency in buildings: windows and daylighting, artificial lighting, computer modeling of building energy use, and infiltration/ventilation and indoor air quality. The general objective of these programs is to develop advanced energy-efficient technologies, computational tools, and experimental facilities. This will allow evaluation of technologies showing the greatest promise for significant energy savings in buildings while maintaining.levels of illumination and air quality adequate for human comfort and health. Specific projects focus on energy-savings opportunities in fluorescent lamps, advanced windows, novel building insulation, analysis of federally assisted housing, residential and commercial building analysis and performance studies, analysis of appliance energy efficiency, and siteplanning studies to minimize summer heat-island effects. An increasing effort is focused on providing more "user friendly" access to the technologies and methodologies developed at LBL. Examples include a graphic user interface to computer codes such as DOE-2, expert systems to aid energyefficient building design, and electronic kiosks for information transfer. Another growing effort involves providing technical assistance to the Federal Energy Management Program (FEMP) as that program seeks to increase the energy efficiency of federal buildings. A third new focus is an emphasis on the development and understanding of integrated building systems, and on assuring their performance (energy, and occupant health and productivity) throughout the life cycle of the building. These projects are undertaken in close collaboration with the building industry and utilities. There is an additional emphasis on supporting the U.S. government's commitment to reducing green-house gas emissions to 1990 levels by the year 2000, by accelerating implementation of new energy-efficient technologies and processes.

Both domestic and international studies of economic impacts of alternative energy policies are expected to grow. The purpose of surveying the conservation policies of other developed countries is to enable the U.S. to compare progress in this area and perhaps adopt some of their effective efficiency measures.

\section{Federal Energy Management Program}

LBL's In-House Energy Management Program, supported by the Federal Energy Management Program (FEMP), pursues opportunities to significantly reduce energy costs at LBL. It is estimated that the program will result in $\$ 750,000$ in annual savings by FY 1995 . The program involves surveys and studies of existing conditions, retrofit projects, new construction, support, and utility management and related operational programs. Retrofit projects have improved the efficiency of mechanical systems, lighting, and utility systems. 


\section{Fusion Energy Program Direction}

Fusion energy research at $L B L$ focuses on accelerator systems supporting the nation's inertial confinement and magnetic fusion energy programs. Materials, superconducting magnets, and magnetic fusion energy theory are important parts of the program. LBL's heavy-ion fusion accelerator research has focused on the physics and technology of induction acceleration as the means for producing high-current, heavy-ion beams as drivers for inertialconfinement fusion systems. In comparison with other possible inertialfusion drivers (e.g., lasers), beams of heavy ions offer important advantages for practical applications, including high efficiency from the "wall plug" to the beam, good potential for beam-to-pellet energy focusing and coupling, and high pulse-repetition rates. LBL's current efforts have resulted in successful completion of the multiple-beam experiment to examine the initial accelerator components for space-charge-dominated beams undergoing current amplification. Current efforts are directed toward design and development of the next set of studies, the Elise accelerator and experiments. The Elise accelerator would constitute a significant step toward realizing induction-accelerator technology that could be used to produce intense sources of spallation neutrons. An induction accelerator that uses 5-MW beams of protons at $1 \mathrm{GeV}$ to generate spallation neutrons in 100-ns bursts at a rep-rate of $10 \mathrm{~Hz}$ would be quite similar to, but one-tenth the size and cost of, an inertial fusion driver. At higher average power levels (i.e., rep-rate), induction accelerators are candidates for accelerator-based pulsed reactors for waste transmutation, for tritium production, and for a fusion material test facility.

LBL has contributed to the magnetic fusion energy program through the development of neutral beams for heating, refueling, and drive current in reactor plasmas. LBL's work on positive-ion-based neutral beams has been coupled with the research efforts at the Tokamak Fusion Test Reactor at Princeton and the DIII-D at General Atomics in La Jolla. The most prominent achievement thus far has been the design, development, and transfer to industry of the Common Long-Pulse Source, which has been incorporated into all major U.S. fusion experiments.

Many proposals for future magnetic-fusion projects involve injection of dc neutral beams at high currents and at high kinetic energy. The beams play a significant role in heating the plasma and in driving the toroidal current noninductively in the steady state. LBL has traditionally been a leading center for design and development of these neutral-beam injection systems, including the standardized Common Long-Pulse Source. Future work is likely to be carried out under the aegis of the Japan Atomic Energy Research Institute (JAERI).

An additional contribution to the magnetic fusion program in the Materials Sciences Division is research on alloys and weldments for lowtemperature superconducting magnets for magnetic-confinement fusion systems. The alloys must withstand extremely high magnetic fields at the cryogenic temperatures needed for superconductivity.

\section{Fossil Energy Program Direction}

LBL conducts research directed toward making coal more usable, including studies on conversion to gaseous and liquid fuels and reduction of emissions. One current effort focuses on the low-temperature catalytic gasification of graphite and other forms of carbon. A flue-gas chemistry 
project is directed toward methods of simultaneous removal of $\mathrm{SO}_{2}$ and $\mathrm{NO}_{x}$, and other new processes are being developed to remove $\mathrm{H}_{2} \mathrm{~S}$ from gas streams, such as those produced during coal gasification. Another project is studying the erosion and corrosion of materials used in systems developed for coal conversion and use.

In the oil and gas area, LBL is completing a study directed to the development of methods for characterizing heterogeneous and fractured reservoirs through a combination of geological, geophysical, and hydraulic studies. Heuristic methods are used to determine the systematic patterns of conductive features that result from the geologic processes. Improved seismic and electromagnetic methods are being developed for imaging these features from boreholes. Both of these types of information are used to obtain an improved model of reservoir performance to enhance our ability to manage reservoir production. LBL is continuing development of advanced inversion codes and interpretation methods. This work has active industrial support.

LBL is now collaborating with three other laboratories in the Oil Recovery Technology Partnership in joining with industry on a cost-shared basis to adapt and develop geophysical and other reservoir measurement and production techniques to aid oil production with a focus on California reservoirs. In FY 1995 this effort will be broadened further in support of DOE's Domestic Natural Gas and Oil Initiative.

LBL is exploring the major factors that will affect the economic and technical success of cogeneration in China, with the object of promoting the cost-effective application of that technology in China.

LBL is also exploring selenium's presence in San Francisco Bay and its effects on the Bay's water quality, as well as assessing whether oils refined in the region are sources of selenium contaminants.

\section{Engineering and Geosciences Program Direction}

The Geosciences Program at LBL is strengthening its multidisciplinary effort to support the scientific basis of many energy-related technologies, including development of hydrocarbon and strategic-mineral resources, safe disposal of radioactive and toxic chemical wastes, and exploitation of geothermal energy. Earth sciences researchers at LBL are among the leading experts in the areas of subsurface imaging of the structure and dynamics of the earth's crust, experimental investigation of the mechanisms by which lithospheric processes influence energy resources, and numerical modeling of geochemical and hydromechanical processes occurring in heterogeneous fractured rock formations.

Geohydrology research at LBL includes experimental studies of the physical behavior of fluid-saturated rock, numerical modeling of coupled processes in subsurface reservoirs, and investigation of the mechanisms associated with chemical transport and multiphase fracture-flow phenomena. New algorithms are being developed to greatly increase the efficiency of the numerical solution of flow and transport equations.

Geophysicists, supported by LBL's Geophysical Measurements Facility and the Center for Computational Seismology, are developing methodologies and instruments to define crustal structure over a range of scales, to monitor processes in subsurface reservoirs, and to track the movement of contaminated plumes in underground aquifers. Borehole seismic and 
electromagnetic source development, coupled with real time signal processing, are keys to improved high-resolution imaging capabilities. At a laboratory scale, new approaches are being employed to understand fracture processes and wave propagation in fractured media. Other geophysical research uses new computational codes to measure fracture properties in subsurface reservoirs and to map hydrofractures at well sites

Geochemical studies focus on the thermodynamic properties of electrolytes, the generation and migration of petroleum compounds, and the interactions between minerals and groundwaters. Analytical capabilities of the Center for Isotope Geochemistry provide a powerful means of characterizing natural systems. This center is an important element in many of the multidisciplinary geological and environmental investigations at LBL. It provides a focus, in collaboration with geophysicists and geologists, for continued study of crustal processes as part of the multiagency Continental Scientific Drilling Program.

\section{Energy Biosciences Program Direction}

LBL's program continues to improve understanding of the unique features of photosynthetic organisms for collecting light energy and storing it as chemical energy. One project uses spectroscopic techniques to map the components and the kinetics of the light reactions. The genetics of the photosynthetic apparatus of single-celled organisms are studied to allow application of DNA-cloning techniques to elucidate photosynthetic mechanisms. The light regulation of gene-encoding components of the photosynthetic apparatus in plant protoplasts is also being investigated. The DOE Division of Energy Biosciences is also supporting research at LBL's Center for Advanced Materials on the enzymatic synthesis of materials.

\section{ENVIRONMENTAL QUALITY}

Environmental quality is a key strategic growth area for LBL, directed toward understanding and reducing environmental, safety, and health risks and developing the technologies and institutions required for solving global and regional environmental problems. Key program areas include environmental management and environment, safety, and health. The following sections identify key LBL initiatives in support of these key areas and describe research and development directions. LBL's environmental quality initiatives include the following:

- Assisting Development of Energy Practices and Technologies

- Energy and Environmental Technology Strategic Initiative

- Advanced Risk-Based Environmental Technologies and Remediation

- California Environmental Enterprise

\section{Assisting Development of Energy Practices and Technologies Initiative}

The Assisting Development of Energy Practices and Technologies (ADEPT) Initiative has helped to establish a new program at DOE that provides assistance to developing and transitional countries in their choice and application of energy technologies. The program supports technology 


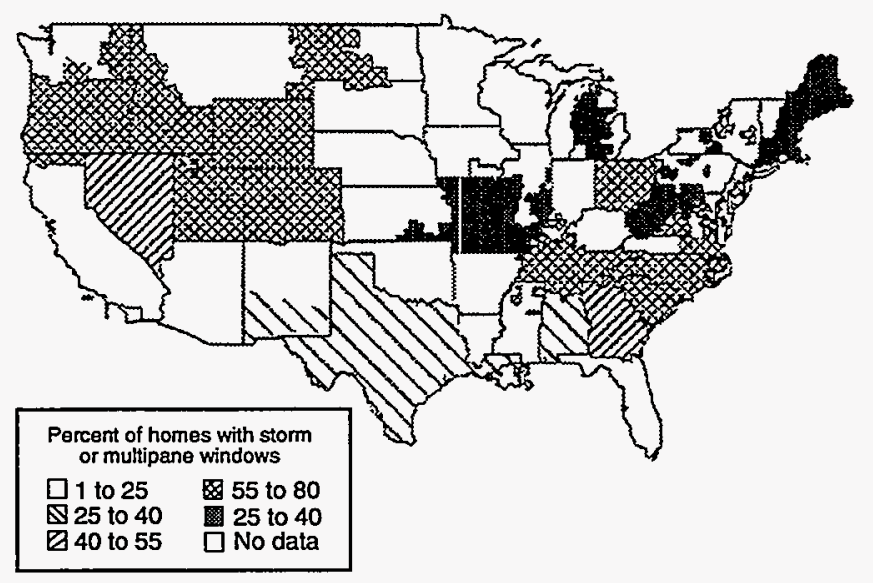

LBL has done a number of studies that support technology choices to produce energy more efficiently, reduce undesirable emissions, and still be economically competitive. For example, this map shows the saturation of homes with storm or multipane windows in the U.S. Such a study is of specific value in a comprehensive assessment of residential retrofit activities. However, it also illustrates the value of evaluating energy-saving conservation measures for improvement of forecasting, program and policy development, industry coordination, and research planning.

choices that-when compared with typical current technology-produce or use energy more efficiently, cause reduced levels of emissions of pollutants and greenhouse gases, and are economically competitive or superior on a lifecycle cost basis.

The new program consists of seven elements: (1) energy technology adaptation, (2) demonstrations, (3) key country programs (including China and India), (4) one-stop shopping network, (5) training, (6) applied R\&D, and (7) institution building in developing countries and Eastern Europe. Initial work on the program began within the Office of Policy, Planning and Education during FY 1993.

ADEPT will be especially valuable in responding to needs of developing countries, the former Soviet Union, and Eastern Europe for technical assistance in limiting greenhouse gas emissions in a highly cost-effective manner. It will also provide important benefits in reducing future global oil demand and in helping to establish international markets for U.S. energy technology. This program is an important United States contribution to support recommendations made by the Intergovernmental Panel on Climate Change and the United Nations Conference on Environment and Development. The program will be conducted consistent with applicable DOE Orders and Regulations. The resources indicated below are for LBL's activities for the national DOE program. Specific research proposals will be reviewed for NEPA and CEQA compliance requirements. 
Assisting Deployment of Energy Practices and Technologies Initiative Resource Requirements $(\$ M)^{a}$

\begin{tabular}{lccccccc}
\hline Category & 1995 & 1996 & 1997 & 1998 & 1999 & 2000 & Total \\
\hline Operating & 0.5 & 1.2 & 1.5 & 2.0 & 2.2 & 2.4 & 9.8 \\
Construction & 0.0 & 0.0 & 0.0 & 0.0 & 0.0 & 0.0 & 0.0 \\
\hline
\end{tabular}

aPreliminary estimate of LBL actual year Budget Authority (B\&R code PE).

\section{Energy and Environmental Technology Strategic Initiative}

Through its interagency report Technology for $A$ Sustainable Future: $A$ Framework for Action (July 1994), the Administration challenged the nation and the world to develop a comprehensive long-term strategy to stimulate economic growth, create skilled jobs in the energy and environmental technologies industries, and improve the quality of life and the environment for future generations. The Department of Energy, through its laboratories and their alliances, provides a substantial portion of U.S. basic and applied science and technology research, which has been critical in the development of innovative energy and environmental technologies and will continue to be so into the future.

Often these technologies are interrelated and connected to policy actions. For example, pollution prevention is a direct benefit of energy efficiency. DOE supports LBL's program to develop and commercialize low-emissivity windows, which now account for approximately 36 percent of the market. Aided by DOE efforts in the development of ratings and labels, the energy-efficient windows have more readily penetrated the market. Use of the windows has resulted in a cumulative energy savings of $\$ 1.6$ billion to date. By the year 2015, use of advanced energy-efficient windows will prevent the addition of 47 million tons of $\mathrm{CO}_{2}, 500,000$ tons of $\mathrm{SO}_{2}$, and 40,000 tons of $\mathrm{NO}_{x}$ into the atmosphere.

LBL has been working with the other national laboratories to identify how such DOE strengths and capabilities can best answer the Administration's call to action. Multilaboratory working groups have been formed in eight focus areas, two of which (water resources and energy efficiency) are led by LBL researchers:

- Air Quality

- Energy Efficiency

- Global Change

- Toxic and Hazardous Waste

- Water Resources

- Natural Resources

- Biodiversity and Ecosystems .

- Natural Disaster Reduction

As the exact scope and nature of the DOE sustainable technology program continue to take shape, it is clear that LBL will contribute substantially to the program. For example LBL's energy efficiency programs will continue to play a major role. In addition, the Toxic and Hazardous Waste 
working group has identified risk-based decision making and hazardous and radioactive site remediation as two of five key areas on which the DOE should focus. LBL's SELECT initiative (see the Advanced Risk-Based Environmental Technologies and Remediation Initiative on the next page) addresses both of these topics.

The Alameda Center for Environmental Technologies (ACET) is another national laboratory-led effort (see Northern California Defense Conversion Initiative p. 4-62) that has anticipated and will implement the principles of the technology for a sustainable future. With ACET's emphasis on green technologies and quality job creation, the Center has benefited during a two-year planning process from broad involvement by stakeholders, including regulatory agencies, community and environmental groups, and environmental companies. In these respects it is a model project for sustainable technology development.

Energy and Environmental Technology Strategic Initiative Resource Requirements $(\$ M)^{a}$

\begin{tabular}{lccccccc}
\hline Category & 1995 & 1996 & 1997 & 1998 & 1999 & 2000 & Total \\
\hline Operating & 1.3 & 2.5 & 5.0 & 5.0 & 5.0 & 5.0 & 23.8 \\
Construction & 0.0 & 0.0 & 0.0 & 0.0 & 0.0 & 0.0 & 0.0 \\
\hline
\end{tabular}

aPreliminary estimate of LBL actual year Budget Authority (B\&R codes EE, PE, EM).

\section{Advanced Risk-Based Environmental Technologies and Remediation Initiative}

The Initiative provides integrated, interdisciplinary methods, technologies, and approaches to support or enable the development of risk-based environmental policy on problems of national importance, such as remediation of subsurface contamination, urban air pollution, and stratospheric ozone depletion. This is a partnership between LBL and the Department of Defense (DOD)/McClellan Air Force Base. A memorandum of understanding is in place whereby McClellan and LBL will "collaborate with regard to environmental research, technology development, and field demonstration of promising environmental technology."

The initial focus of the collaboration is the development of a methodology and user-friendly software, called SELECT, for evaluating and comparing environmental remediation technologies with respect to their risk reduction and cost effectiveness. The software builds upon and integrates existing codes for various sub components of the methodology, such as transport and transformation of multimedia contaminants, background and siterelated exposures, and relative ranking by carcinogenic hazard for chemicals of concern. Key components of the research program are:

- Subsurface site characterization data from contaminated sites at McClellan are being used to test and advance subsurface fate and transport models.

- Exposures to volatile organic compounds (VOCs) and heavy metals will be modeled for people living near the base, base personnel, and site-cleanup workers. Multiple exposure pathways also will be modeled. 
- Cancer risk estimates will be developed based on linear extrapolation from rodent cancer tests. We propose to reduce uncertainty by addressing gaps in the current process.

- To improve remediation effectiveness, technologies and strategies will be identified according to their potential for risk reduction and cost effectiveness.

- To ensure that there is a net reduction in health risk, the secondary effects of any chosen remediation will be evaluated. Exposure reductions anticipated as a result of various remediation technologies employed at the most hazardous sites will be calculated.

This project will develop a new, integrated science-based methodology for use on information networks to facilitate more risk-reducing and cost effective environmental remediation. A broadened perspective on cancer risk will be provided by ranking and comparing possible hazards from exposures to site contaminants with background exposures to naturally occurring chemicals in the diet and with other exposures to synthetic chemicals in indoor and workplace settings. The methodology will also advance and integrate scientific understanding of exposure pathways and human health effects with environmental remediation. Specific research proposals will be reviewed for NEPA and CEQA compliance requirements.

\section{SELECT: Risk-Based Cleanup}

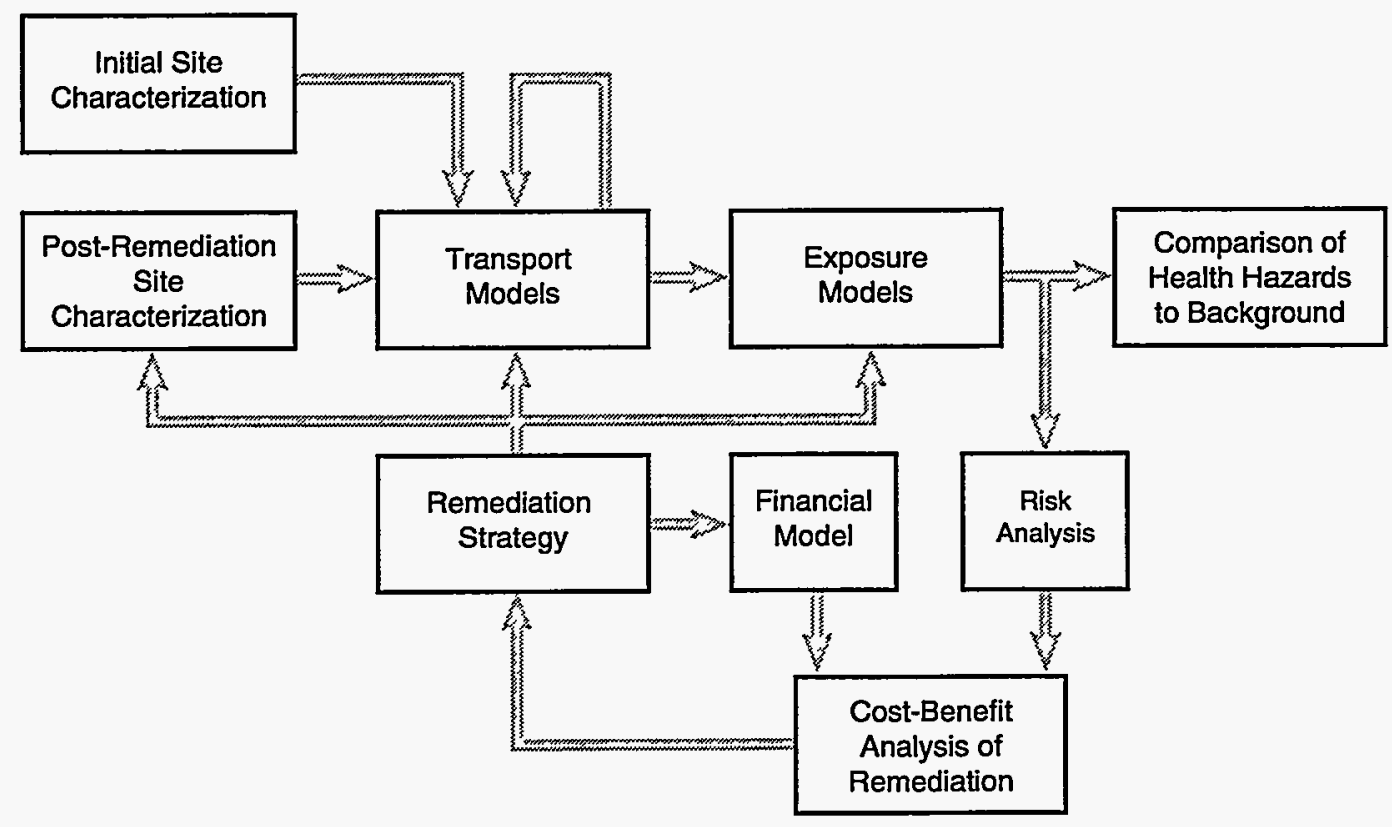

SELECT is a risk-based environmental assessment tool being developed at LBL. The tool will enable cleanup decisions to be based on cost-effective risk reduction. SELECT modules can be updated individually to reflect current scientific understanding. 
Advanced Risk-Based Environmental Technologies and Remediation Initiative Resource Requirements $(\$ M)^{a}$

\begin{tabular}{lccccccr}
\hline Category & 1995 & 1996 & 1997 & 1998 & 1999 & 2000 & Total \\
\hline Operating & 1.5 & 1.7 & 1.9 & 2.1 & 2.3 & 2.5 & 12.0 \\
Construction & 0.0 & 0.0 & 0.0 & 0.0 & 0.0 & 0.0 & 0.0 \\
\hline
\end{tabular}

apreliminary estimate of $L B L$ actual year Budget Authority ( $B \& B$ codes $E M, K P)$.

\section{California Environmental Enterprise Initiative}

LBL has joined with LLNL and SNL to form a joint project called the California Environmental Enterprise (CEE). Formed in FY 1994 with initial funding from the Office of Technology Development, Office of Environmental Restoration and Waste Management, the CEE project is seeking to help facilitate the cleanup of the many contaminated properties in California by utilizing the environmental remediation expertise developed at the DOE laboratories.

LBL's role in the CEE will be to use the special expertise within its Earth Sciences, EH\&S, Engineering, and Information and Computing Sciences Divisions for the following activities: (1) to assist small environmental technologies businesses gain access to information about technologies being developed within LBL and other federal laboratories, (2) to broker technologies developed within the DOE programs to companies in California, and (3) to help design and develop a better information system that will give businesses, regulators, and laboratories access to a wider range of information than is currently available from a single source.

These activities will be coordinated through a CEE project office, whose organizational structure is being designed by the laboratories and DOE to help meet programmatic needs. The CEE will partner with umbrella nonprofit environmental organizations such as ACET (see Northern California Defense Conversion Initiative, p. 4-63).

California Environmental Enterprise Initiative Resource Requirements $(\$ M)^{\mathrm{a}}$

\begin{tabular}{llllllll}
\hline Category & 1995 & 1996 & 1997 & 1998 & 1999 & 2000 & Total \\
\hline Operating & 0.25 & 0.3 & 0.35 & 0.4 & 0.4 & 0.5 & 2.2 \\
Construction & 0.0 & 0.0 & 0.0 & 0.0 & 0.0 & 0.0 & 0.0 \\
\hline
\end{tabular}

apreliminary estimate of LBL actual year Budget Authority (B\&B codes EM).

\section{Environmental Management Program Direction}

\section{Policy, Planning, and Analysis}

LBL undertakes analysis activities in support of policy issues of concern to DOE. Recent efforts include analysis of "feebates" (an imposition of fees and concomitant offering of tax incentives to encourage energy-efficient technologies) as a policy approach to increase auto fuel economy, combustion pollution exposure that takes place indoors, the development of data and models for developing projections of energy demand under a variety of 
policy cases, and assessment of a variety of policies as an input to DOE's assessment of U.S. energy strategies.

LBL is supporting (along with Battelle/Pacific Northwest Laboratory) the creation of the Beijing Energy Efficiency Center. This center has the mandate to influence Chinese policy to promote energy efficiency, to carry out training and educational programs, and to promote trade and joint ventures in energy-efficiency products.

In 1994, the U.S. government initiated a program to provide technical assistance to developing countries in their efforts to find ways to reduce the growth of greenhouse gas emissions. While the program is governmentwide, the Policy Office is playing a leading role in organizing this enterprise. LBL has been selected as the organization that will organize the technical assistance effort, using our own expertise and that of other organizations. This will begin with assistance to 14 countries and is expected to expand to about as many more countries over time.

\section{Environmental and Health-Effects Research}

Environmental research at LBL comprises multidisciplinary efforts on global, regional, and local environmental problems such as epidemiological factors, subsurface contamination, indoor air quality, and high-magneticfield environments, all conducted for the Office of Health and Environmental Research.

The Laboratory is advancing programs in support of DOE's research initiatives on the subsurface environment. The Laboratory's proposed program encompasses the biological and hydrogeochemical control and remediation of toxic waste. Specific projects include characterization of contaminants, subsurface heterogeneity, transport processes, and enhancement of restoration methods. Improved risk-estimation methods will enable the deployment of cost-effective remediation technologies.

LBL is developing an interdisciplinary program to investigate the processes that lead to changes in the physical and chemical characteristics of the atmosphere and other potential changes in the ecosystem. Initial research subjects include atmospheric processes that are involved in the generation of nucleating particles from artificial and natural sources; heterogeneous chemical processes, the role of particulates in the formation of clouds, and the resulting chemical and physical changes in the atmosphere; and atmosphere-ecosystem interactions.

The LBL program on radon migration includes unique experimental facilities for studying radon movement in soil and into buildings under controlled conditions. These studies will allow comparisons with detailed numerical simulation models. In addition, the radon research program examines the effects of other parameters, including other indoor air pollutants, building construction and operation, and climate conditions, on the resulting indoor radon concentrations and, ultimately, on the radiation doses to the building occupants from these indoor exposures.

\section{Environment, Safety, and Health}

LBL is continuing its strong programs in analytical methods development and statistical studies of environmental and epidemiological factors supported by the Office of Epidemiology and Health Surveillance. The Population at Risk to Environmental Pollution Project focuses on the collection, analysis, and interpretation of data pertaining to relationships between 
human health and environmental pollution. Computational techniques are developed for the analysis of ecologic data, especially small-area geographic data, to investigate alleged departures from expected disease rates, to generate etiologic hypotheses, and to plan clinical trials or cohort studies. The role of the Comprehensive Epidemiologic Data Resource (CEDR) Project is to provide exposure and health data on DOE workers to research epidemiologists both within and outside DOE. CEDR includes work on past and current epidemiologic studies by DOE and the Department of Health and Human Services, and is being expanded to include current epidemiologic monitoring data on DOE employees. Other data sets of interest, such as Radiation Effects Research Foundation summary tables and dose reconstruction data from the Nevada Test Site, are included. The goal of the program is to encourage independent review and augmentation of existing findings on DOE worker health effects, in response to criticism that DOE research has not been viewed as impartial by the public. The availability of CEDR data has been increased by posting the data on the World Wide Web and providing access through Gopher and Mosaic interfaces.

\section{Work For Others-Environmental Protection Agency}

LBL conducts research on the hydrogeological transport of contaminant plumes from deep underground injection disposal. In the area of global environmental effects, LBL is characterizing the emissions of energy technologies, improving global energy projections, fostering international awareness of global trends, studying effects of tropical deforestation, and gathering information on the potential effect of global climate change on U.S. natural resources. LBL, with other national laboratories, is working to develop new programs in partnership with the Environmental Protection Agency (EPA) that advance national environmental goals, including the more efficient use of energy to reduce greenhouse gas emissions.

\section{Environmental Characterization Program Direction}

\section{Engineering and Geosciences}

The thrust of $L B L$ research will be in two different areas, the understanding of radionuclide chemistry and mixed organic radionuclides and the effect of natural subsurface heterogeneity on microbed behavior.

The goal of this research is to provide fundamental knowledge on the complexation behavior of neptunium, plutonium, and cobalt with synthetic organic-radionuclide wastes in the subsurface at DOE sites. Fundamental knowledge of the chemical processes that occur between radionuclides and synthetic organic chemicals present in DOE waste sites will provide the ability to more accurately predict the processes that control contaminant mobilization and transport in the subsurface environment. We are conducting experiments to determine the thermodynamic and kinetic solution phenomena such as complexation constants, speciation, solubility of complexes, and the nature of compounds that can form between constituents of mixed organic-radionuclide wastes.

The heterogeneity research is part of the Subsurface Science Program Heterogeneity Subprogram. It contributes to meeting the goals of the subprogram by improving means to relate subsurface physical properties to the microbial heterogeneity of natural systems. A large number of field- 
scales of heterogeneity range from the pore scale to the bed scale and beyond. To relate the significant scales of variation of microbial and chemical behavior to the variation of physical properties, it is critical to know the scales at which various processes dominate. Without this knowledge, it would be impractical to attempt to relate a small-scale process (i.e., microbial behavior) to field-scale measurements.

The goal of the work is to use subsurface imaging to identify the fundamental scales of variation of physical parameters that control transport behavior relative to predicting subsurface microbial behavior. With this information, it may be possible to relate physical and chemical heterogeneity (i.e., those parameters that geoscientists have experience measuring in situ) to significant microbial heterogeneity and thus explain and predict their behavior in the subsurface. This approach uses controlled meter-scale field sites and supplementary laboratory and intermediate-scale information to characterize those physical properties that affect fluid flow and chemical transport and can be imaged with in situ methods. The work must be tightly integrated with chemical and microbial characterization and process definition efforts that will be undertaken in the subprogram. At DOE sites where information has been gathered on the microbial and chemical properties, field work will be carried out to define and characterize the natural subsurface physical heterogeneity. This work is being done in close collaboration with Pacific Northwest Laboratory, Oak Ridge National Laboratory, and the University of Virginia as part of DOE's Subsurface Science Program for studying subsurface heterogeneity and bacterial transport.

\section{Civilian Radioactive Waste Management}

LBL continues a strong multidisciplinary program of interrelated geoscience and geological engineering research important to the safe long-term underground storage of high-level nuclear wastes, e.g., characterization of deep geologic formations, determination of the physical and chemical processes occurring in the repository rocks, analysis of hydrologic and chemical transport mechanisms, and development of predictive techniques for repository performance. Coupled with ongoing basic research, LBL is contributing to technology and applied development research at DOE's Yucca Mountain Project as well as to international projects in cooperation with Sweden, Switzerland, Canada, and Japan.

Experimental work involves testing rock samples to determine fundamental chemical, mechanical, and hydrologic parameters under a suite of anticipated repository conditions. Complementary research is conducted on the solubilities of actinides and the characteristics and processes that control radionuclide transport in host rocks. Related efforts involve the understanding of processes and the development of methods for predicting the response of geologic systems to repository development and the performance of geologic environments for various repository containment designs. These expanding research activities draw upon LBL's expertise in chemistry, earth science, computing, and numerous engineering fields.

\section{Work For Others}

Laboratory scientists are investigating the geochemistry of selenium and other trace elements at Kesterson Reservoir, which has been a terminus of agricultural drainage water in California's San Joaquin Valley. Continuing 
collaborative investigations are under way to evaluate remediation techniques for the area's soil. Related research is being conducted at Stillwater Marsh, Nevada.

The Agency for International Development is supporting a multiyear effort in which LBL will perform research in support of improving the efficiency of energy use in developing countries.

The Laboratory conducts research for the Electric Power Research Institute (EPRI). Chemistry-related research includes studies on reducing oxidation and scale formation, and on oxygen depletion in compressed-air storage. Another EPRI project is the study of surface modification with metal plasma techniques.

The Gas Research Institute supports databases on the influence of clays on seismic-wave attenuation in reservoir rocks. The California Air Resources Board is sponsoring an analysis of polycyclic aromatic hydrocarbons in indoor air.

LBL's expertise in buildings is recognized by the California Energy Commission and the energy utilities. Much of the support is through the California Institute for Energy Efficiency (CIEE), a joint effort among the California energy utilities, LBL, the University of California, and the California Energy Commission and Public Utilities Commission. CIEE manages a focused research program, with the research carried out by California universities and DOE national laboratories located in the state. CIEE's overall research framewrork is determined by a research board consisting of high-level executives from the utilities, the University, the commissions, DOE, the Gas Research Institute, and EPRI. A planning committee with members from these same institutions approves individual research projects within this framework. CIEE serves in effect as a Work-for-Others (WFO) agency that evaluates and funds proposals from LBL along with those from other California research institutions. Particular LBL projects funded by CIEE include the study of efficient systems for thermal distribution in buildings, integrated envelope and lighting technologies, end-use technology performance data, urban landscape modifications to reduce energy use and air pollution, and advanced combustion devices to reduce nitrogen oxide emissions.

Additional utility support is as follows: Southern California Edison supports window studies; Pacific Gas and Electric Company supports a study of end-use energy intensifiers in commercial and residential buildings; and the Sacramento Municipal Utilities District supports energy-savings studies of shade trees and other surfaces.

\section{Environmental Restoration and Waste Management Program Direction}

The Laboratory is implementing site projects for restoration and waste management consistent with DOE's National Environmental Restoration and Waste Management Program. Through direct involvement at LBL and other DOE and DOD sites, LBL intends to identify major technology gaps in environmental restoration. These gaps will then be addressed and resolved in close cooperation with industry. In addition, and as described in greater 
detail in Section $V$, the existing and budgeted site projects address specific conditions at the Laboratory, including facilities and operating programs for corrective actions, environmental restoration, and waste management.

In support of the Office of Technology Development in DOE's Environmental Restoration and Waste Management program, and to facilitate the development of underlying science conducted in the Offices of Basic Energy Sciences and Health and Environmental Research, LBL is developing a multidisciplinary research and development program directed at improving the effectiveness and cost/risk benefits of environmental restoration technologies. The program has four components:

- Improved characterization of subsurface environments, including better measurement of their biological, chemical, and physical properties and better definition of the associated contaminant transport processes.

- Development of methods for assured containment and control of subsurface biological, chemical, and radiological contaminations.

- Development of advanced remediation technologies, including instrumentation for cost-effective measurements and treatment methods appropriate to complex and heterogeneous subsurface environments.

- Improved risk assessment and prioritization systems to allow better allocation of remediation funds.

The methodologies used in the program will include field testing and tracking contaminant fronts; developing descriptive and predictive mathematical models; characterizing heterogeneous underground systems; designing, demonstrating, and testing containment and cleanup systems at specific contaminant sites; and determining the underlying chemical, biological, and thermodynamic properties involved in mixed contamination. As program plans are developed, they will be reviewed for NEPA and CEQA documentation requirements.

\section{I.] INDUSTRIAL COMPETITIVENESS}

Industrial competitiveness efforts of the Laboratory promote economic growth and the creation of high-technology jobs through research and development partnerships with industry. They can also help industry become more competitive by cost-effectively shifting from inefficient technology, processes, and activities to resource efficiency and pollution prevention. LBL's activities support DOE's efforts toward developing partnerships to advance this core business area. The following sections identify four important LBL initiatives and describe research and development directions.

- Advanced Lithography

- Fabrication of Microelectromechanical Systems, Using Deep X-ray Lithography

- AMTEX: The AMerican TEXtile Partnership

- Northern California Defense Conversion 
Point diffraction interferometry, a new technique for at-wavelength extreme ultraviolet (EUV) optics testing, uses spatially coherent undulator light from the Advanced Light Source to evaluate multilayer mirrors and other EUV and $x$-ray optical components. When light from an optical component under testing illuminates a specialized mask, the resulting interferograms yield information about the quality or defects of the optical component. This image is an interferogram that was taken using $122-\AA$ light, with the pinhole of the mask placed somewhat off-axis and out of the zone plate focal plane.

\section{Advanced Lithography Initiative}

Since the creation of the first integrated circuit in 1960, there has been an ever-increasing density of devices manufactured on semiconductor substrates. The very-large-scale integration (VLSI) era from the mid-70s to the present has seen chip densities increase from 100,000 transistors per chip to over 1 million per chip. The increasing device count was accompanied by a shrinking minimum feature size, from $2 \mu \mathrm{m}$ in the late $70 \mathrm{~s}$ to less than $0.5 \mu \mathrm{m}$ in current 4 -megabit DRAMs. The challenge to continued U.S. industrial competitiveness in microelectronics is the development of techniques of lithography and pattern transfer at minimum feature sizes less than $0.25 \mu \mathrm{m}$. This will lead to densities of over $10^{9}$ transistors per chip. The advanced lithography program at LBL's CXRO focuses on the enabling technologies essential for soft x-ray (SXR) and extreme ultraviolet (EUV) optical imaging systems. These systems will be required for $0.1 \mu \mathrm{m}$ features, implying 1-Gbit DRAM's by the year 2000. CXRO's lithography program will concentrate on the development of EUV/SXR interferometry for atwavelength $(130 \AA)$ testing of optics, nanofabrication facilities for zone plates and reflective masks, high-precision metrology, and the necessary processing for pattern transfer.

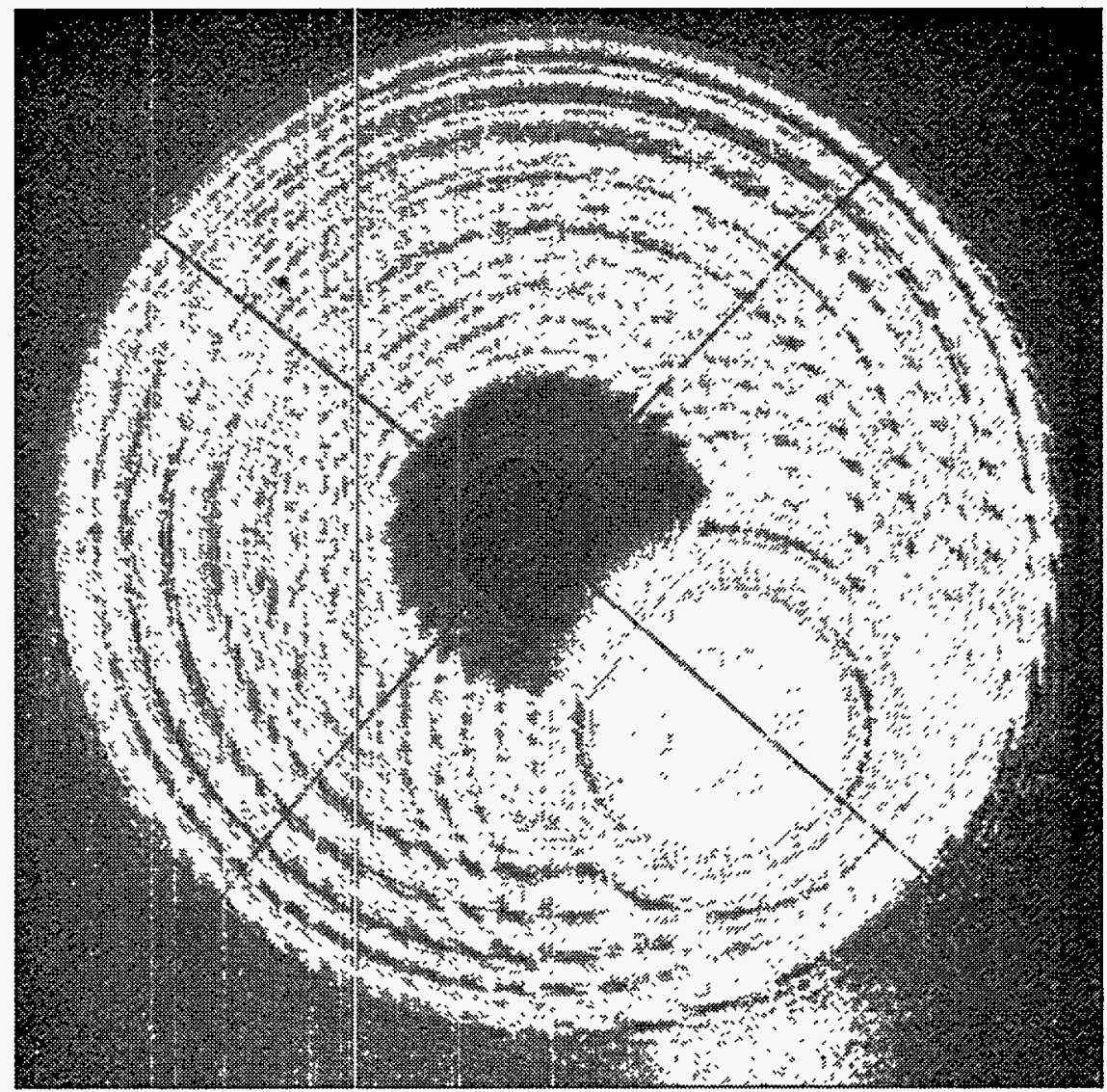

LBL's initiative responds to programmatic needs established by both the Department of Defense and the Department of Energy to provide collaborative support for this emerging area of science, which supports essential U.S. technological capabilities. A successful program requires an integrated consortium of industrial, university, and national laboratory scientists to 
break new scientific and technical ground well in advance of the competition and to train a new generation of scientists to carry the project to fruition in the 21 st century. The consortium will require dedicated laboratory research facilities with modern high-brightness partially coherent $x$-ray and EUV sources, expertise in short wavelength nanometer-scale structures, and the synthesis of new materials whose structures are controlled at the atomic level. Strong participation of a consortium of local microelectronics companies will ensure quick and effective transfer of all relevant technologies.

The ALS, a unique national resource for EUV interferometry and metrology, will be utilized by the collaboration. Additional laboratory equipment dedicated to advanced lithographic research for nanoelectronics applications also will be required. These facilities will include insertion devices, bend magnets, and beamline components; metrology stations to test all optical surfaces and coatings; and advanced EUV interferometers for testing optical surfaces and integrated optical systems, as well as equipment that will support the fabrication of nanostructures and the synthesis of artificially structured materials. Specific initial elements are to include:

- A high-brightness coherent undulator beamline for at-wavelength EUV interferometry

- A direct-write electron beam "nanowriter" for diffractive optics and mask patterning

- Component and system EUV interferometry

- An EUV metrology bending magnet beamline for absolute radiometry and calibrations

A continued investment would further the consortium efforts and lead to improved American competitiveness in this critical technological area. Costs include operational procedures and equipment design in compliance with DOE ES\&H orders and regulations. As proposals under this program are developed, they will be reviewed for NEPA and CEQA documentation requirements.

Advanced Lithography Initiative Resource Requirements $(\$ M)^{a}$

\begin{tabular}{lccccccc}
\hline Category & 1995 & 1996 & 1997 & 1998 & 1999 & 2000 & Total \\
\hline Operating $^{\mathrm{b}}$ & 15.0 & 8.0 & 8.0 & 7.0 & 3.0 & 0.0 & 41.0 \\
Construction & 0.0 & 0.0 & 0.0 & 0.0 & 0.0 & 0.0 & 0.0 \\
\hline
\end{tabular}

aPreliminary estimate of LBL actual year Budget Authority from DOD. ${ }^{\text {b }}$ Costs are primarily for equipment.

\section{Fabrication of Microelectromechanical Systems, Using Deep X-ray Lithography Initiative}

Microelectromechanical systems (MEMS) represent the next phase of the microfabrication revolution. These systems are made by applying processing techniques developed for the semiconductor industry to the design of microscopic sensors, actuators, and motors. This technology provides a 
powerful tool for mass production and miniaturization of mechanical systems into a dimensional regime not accessible by traditional machining operations. The synergy with integrated circuit manufacturing provides the potential for integration of mechanical systems with the associated electronics required for closed-loop control.

One technique specifically developed for the creation of high-aspectratio devices is called LIGA (a German acronym for Lithographie Galvanoformung, Abformung). LIGA is a combination of deep x-ray lithography, electroplating, and injection-molding processes, which allows the fabrication of microstructures with lateral dimensions in the micrometer range, vertical dimensions of several hundred micrometers, and submicron tolerances. This type of $x$-ray lithography depends on the existence of synchrotron radiation sources that can provide a sufficient flux of highly parallel $x$-rays in the 3-10 KeV range. The ALS is an excellent source of radiation for this application. The techniques of deep $x$-ray lithography are used in the construction of micromotors, acceleration sensors, microgears, and linear comb drives. The technologies we will develop in support of micromechanical fabrication are:

- Design and construction of a bending magnet beamline with three branchlines. The branchlines will be configured as follows:

- A branchline fitted with aspheric optics for the exposure of large areas. An exposure station with sufficient throughput and ease of use for advanced industrial prototype development.

- A branchline fitted with a planar mirror for development of exposure systems.

- A white-radiation beamline for resist exposure modeling.

- Development of electroplating techniques that address the issues of plating stress and thickness.

- Improvement of the properties of resists used in deep x-ray lithography.

- Design and fabrication of novel micromechanical devices that exploit the properties of deep x-ray lithography.

This technology will be used in the manufacture of microsensors and actuators that will revolutionize automobile, aerospace, and medical technology in the same manner that integrated-circuit process technology revolutionized the electronics industry. The processes and devices addressed by our program will seek solutions to the basic problems that currently slow the commercialization of this technology. The resulting impact will be a new class of miniaturized components such as micromotors, sensors, actuators, pumps, valves, and interconnects that will be used to manufacture commercial products. Specific proposals will be reviewed for NEPA and CEQA compliance requirements. 

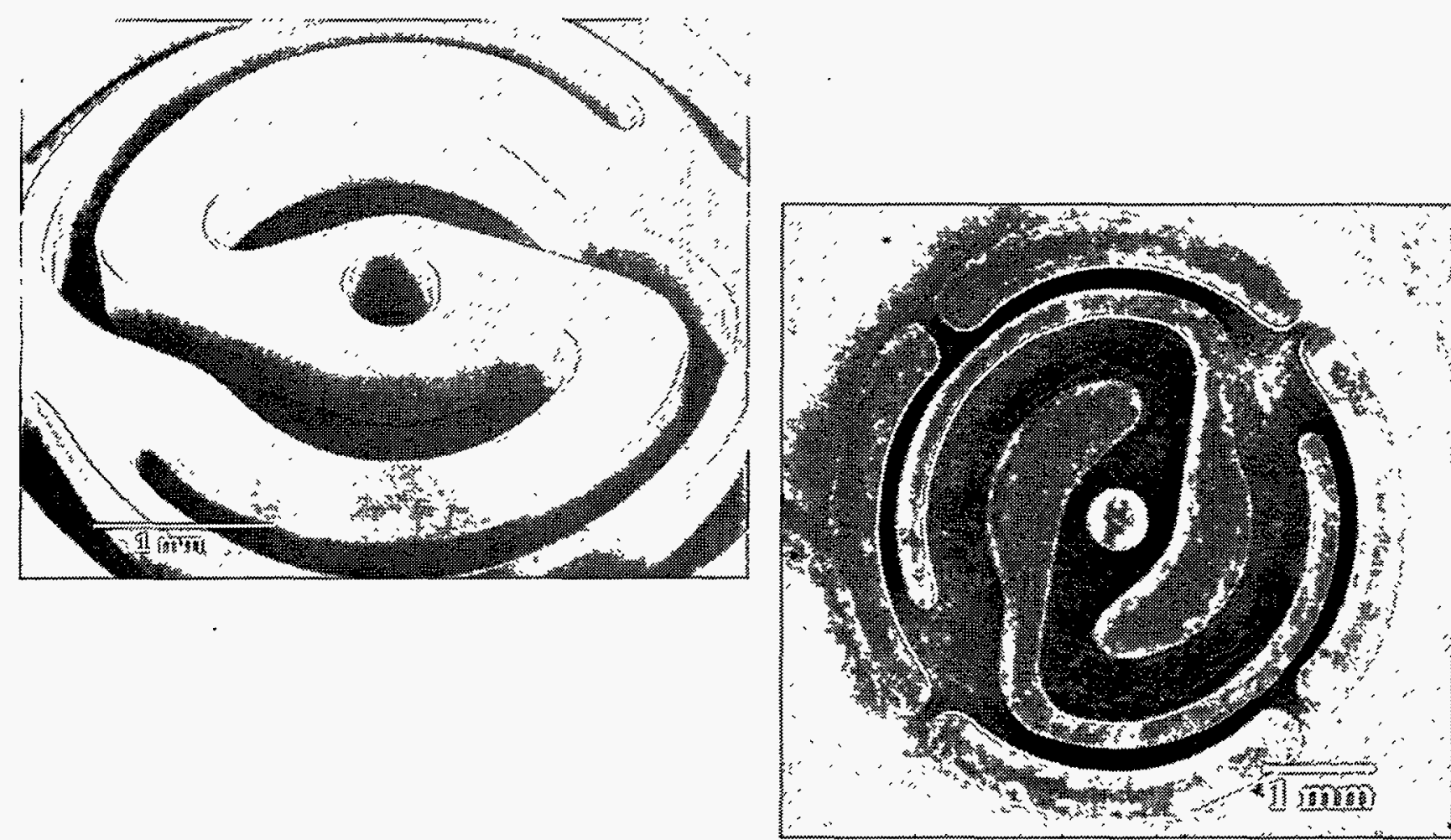

Microfabrication using deep-etch $x$-ray lithography demands the intense, well-collimated sources of $x$-rays provided by the ALS. The high penetrating power of ALS X-rays in the 3 to $9 \mathrm{keV}$ range allows the fabrication of miniature components with vertical dimensions up to the millimeter range and lateral dimensions as small as 5 to 10 microns. Such high-aspect-ratio design improves the performance range of its products. For example, it increases the available torque from micromotor parts. These are photos of two steps in the microfabrication process. The photo on the left shows an 800- $\mu \mathrm{m}$-thick template for a

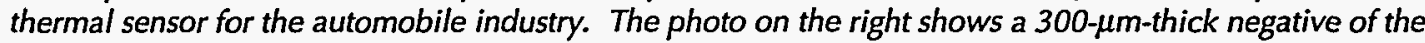
resist template, created by electroforming copper into the template. The copper mold can be used to mass-produce thermal sensors. These microstructures were produced by researchers from $L B L$, the jet Propulsion Laboratory, and SNL.

Fabrication of Microelectromechanical Systems, Using Deep X-ray Lithography Initiative Resource Requirements $(\$ M)^{\mathrm{a}}$

\begin{tabular}{lccccccc}
\hline Category & 1995 & 1996 & 1997 & 1998 & 1999 & 2000 & Total \\
\hline Operating & 2.2 & 2.0 & 0.0 & 0.0 & 0.0 & 0.0 & 4.2 \\
Construction & 0.0 & 0.0 & 0.0 & 0.0 & 0.0 & 0.0 & 0.0 \\
\hline
\end{tabular}

aPreliminary estimate at LBL actual year Budget Authority from DOD.

bTotal including \$1M FY 1993 funding for beamlines.

\section{AMTEX: The AMerican TEXtile Partnership Initiative}

AMTEX is a partnership among the integrated U.S. textile industry and their suppliers (the Industry), the nation's textile research universities, the DOE, and the DOE national laboratories. The Industry encompasses fiber producers, textile and fabric manufacturers, apparel and home furnishings manufacturers, industrial products manufacturers, and retailers of such 
products. It is an initiative in support of DOE's mission to fuel a competitive national economy through technological improvements that enhance industrial competitiveness.

The Industry is among the largest contributors to the national economy. It is the largest employer in the U.S. manufacturing sector and has a long history of excellence in product development. However, today the Industry is being seriously threatened by offshore competition. The rise of imports has accounted for a loss of over 400,000 jobs in the last 10 years. Unless trends are reversed, it is predicted that another 500,000 jobs will be lost over the next 10 years and the Industry may eventually be lost.

The initiative is composed of a large variety of R\&D efforts. Projects were selected jointly by representatives from the national laboratories and members of the Industry. The Demand Activated Manufacturing Architecture (DAMA) Project was identified as a first priority for the initiative. The DAMA Project seeks to facilitate step-change improvements in the competitiveness of the Industry by designing information systems and business practices that will enable more effective and responsive business decisionmaking strategies. Several other projects are funded or are in line for funding: computer-aided fabric evaluation, rapid cutting of fabrics, advanced sensors for sewing and garment identification, cotton genome research, and environmental effects of textile processes.

Lawrence Berkeley Laboratory provides research excellence in many of these areas. $L B L$ is leading the Automation Technical Area in which the rapid cutting and sensors for sewing and garment identification projects are being organized. Current participation in the DAMA project is in the leadership of the Enterprise Understanding Task. The purpose of the Enterprise Understanding Task is to understand the industry as a whole-its structure, its dynamics, its limitations, and the future structures that will make it more effective-and to introduce communication technology, where appropriate, to improve the timeliness of response and enhance industrial coupling.

A novel and important aspect of this initiative is the coupling of the DOE and its resources with an entire industrial complex. The partnership involves all of the national laboratories and an industry represented by industrial supported technology centers that provide a focal point for technology developments and university involvement. A strong approach to teaming, and flexibility in the mechanisms for such teaming, will be the key to the success of this initiative. It represents a new business practice for both the national laboratory system and the integrated textile industry. The joint effort can bring about step-change in performance of the Industry if the talents of the national laboratory system are successfully fused with the talents of an excellent U.S. business system and work force. This powerful synergy can be a role model for revitalizing entire sectors of the U.S. economy. Specific proposals will be reviewed for NEPA and CEQA compliance requirements. 
AMTEX Initiative Resource Requirements $(\$ M)^{a}$

\begin{tabular}{lccccccc}
\hline Category & 1995 & 1996 & 1997 & 1998 & 1999 & 2000 & Total \\
\hline Operating & 2.8 & 4.0 & 5.3 & 6.0 & 8.0 & 8.0 & 34.1 \\
Construction & 0.0 & 0.0 & 0.0 & 0.0 & 0.0 & 0.0 & 0.0 \\
\hline
\end{tabular}

\section{Northern California Defense Conversion Initiative}

The Northern California Defense Conversion Initiative addresses the economic impact of military base closures using LBL strengths in science and technology development, planning, and public service. This initiative is an example of the new working relationships that are being built to bring the power of world-class science and technology to bear on the issues of environmental remediation, manufacturing, and defense conversion. The initiative addresses the intersection of three core DOE businesses-industrial competitiveness, environmental quality, and science and technology.

Closure of the Naval Air Station, Alameda, provides an opportunity to create needed businesses and jobs for the Bay Area region to offset the potentially devastating impact of such base closures by capitalizing on the area's unique research and industrial entrepreneurial resources for technology commercialization and development. The Alameda Reuse and Redevelopment Authority has requested funds from the DOD to initiate a planning study for an institute for Technology Development (ITD) and to investigate the institute's relationship to an associated Industrial Park. The request also calls for implementation of the Alameda Center for Environmental Technologies (ACET), a nonprofit corporation that has been created to expedite the development, commercialization, and application of new environmental technologies. The ITD, Industrial Park, and ACET together will form the Alameda Science City (ASC), an essential part of the integrated Community Reuse Plan for sustainable development of the Naval Air Station, Alameda, site. The Planning Study for the ITD will be undertaken by the University of California on behalf and at the request of the Alameda Reuse and Redevelopment Authority.

LBL will play an active role with the University in the ITD Planning Study. As presently envisioned, the ITD will carry out applied science projects in close cooperation with industries in areas of high national interest, such as manufacturing competitiveness and work force training. The IP will be home to advanced technology industries of various sizes and specialties. The ITD is separate from the IP, but will work in concert with the IP and serve as a magnet to attract industries to the area. The ITD and its component entities will be planned and structured to complement existing institutions in the region. For example, the relationships between these institutions at Alameda and existing national laboratories and university campuses in the region will be fully developed, especially with regard to the size and functions of the ITD. Unnecessary duplication of locally available resources will be avoided. It is anticipated that for the first three to five years after initiation of the ASC project, prior to completion of needed new facilities at the site, the ITD will operate as an intermediary between inter- 
ested ASC participants and existing Bay Area organizations, such as LBL, in facilitating joint ventures.

The ITD and ACET will be dedicated to narrowing the research-commercialization gap, catalyzing new business structures and financing arrangements, and emphasizing science and technology as a nucleus for joint ventures beyond the means of a single large company. These two organizations also will act together as a business incubator with a capacity for laboratory and field studies for emerging environmental businesses, as a liaison between small entrepreneurial enterprises and venture capital, and as a center for vocational training and education.

ACET was developed by the Alameda County Economic Development Advisory Board and the East Bay Conversion and Reinvestment Commission in concert with the Alarneda Reuse and Redevelopment Authority. All three national laboratories located in Alameda County - LBL, LLNL, and SNLwere involved in its development, together with governmental agencies, community and environmental groups, and environmental companies. ACET's areas of expertise will include environmental remediation; ecological restoration of impacted lands; instrumentation for environmental monitoring; waste prevention, handling, treatment, and management; water use, purification, and reuse; environmentally conscious manufacturing; green building technologies; and lessening environmental impacts of transportation. It will supply services in directed technology development, technology demonstration, business assistance, environmental policy and land-use planning analysis, and environmental training and retraining.

This initiative also covers LBL's work with other closing bases, including the Mare Island Naval Shipyard, Fort Ord, and the Presidio. At the Mare Island Naval Shipyard, LBL is evaluating the toxicity and restoration potential of contaminated sites in collaboration with UC Davis. The focus for reuse at Fort Ord is on development of a multi-institutional Center for Science, Technology, Education, and Policy. We have also been involved in a multilaboratory proposal to design innovative groundwater monitoring for the Fort Ord groundwater cleanup. At the Presidio, LBL has contributed to preparation of the energy vision and program, the design considerations for maximum energy conservation in buildings, and an institute to support sustainable technology.

Northern California Defense Conversion Initiative Resource Requirements $(\$ M)^{a}$

\begin{tabular}{lccccccc}
\hline Category & 1995 & 1996 & 1997 & 1998 & 1999 & 2000 & Total \\
\hline Operating & 0.3 & 0.5 & 0.5 & 0.5 & 0.5 & 0.5 & 2.8 \\
Construction & 0.0 & 0.0 & 0.0 & 0.0 & 0.0 & 0.0 & 0.0 \\
\hline
\end{tabular}

aPreliminary estimate of LBL actual year Budget Authority from DOD, to be contracted indirectly.

\section{Industrial Collaborations Program Direction}

LBL's Center for Advanced Materials (CAM) will continue major research efforts selected because of their potential impact on U.S. industrial strength. The research focus evolves with new discoveries and with the changing needs of U.S. industry for fundamental research to underlie its development and commercialization activities. 
- The Surface Science and Catalysis Program focuses on studies of advanced catalysts for clean fuels, pollutant reduction and methanol synthesis, and the atomic-scale surface structure and chemical and mechanical properties of solids and adsorbed monolayers. Advanced surface instrumentation includes nonlinear optical techniques, scanning tunneling and atomic-force microscopies, and advanced Raman spectroscopy.

- The Electronic Materials Program focuses on theoretical and experimental studies of basic materials problems pertinent to the development of advanced electronic and optical materials. Semiconductor thin-film crystal growth and characterization, and comprehensive investigations of structural properties of heterointerfaces are pursued.

- The Polymers and Composites Program pursues polymer synthesis and studies of the relationships between polymer processing and microstructure, focusing on anisotropic materials and surface interactions between polymer liquids and metals.

- The High-Performance Metals Program addresses the mechanisms of structural failure, including fatigue and fracture in metals and intermetallics and is concerned with the theoretical and experimental studies of the properties and development of metal alloys, formable steels, advanced intermetallics, and materials for high-field superconducting magnets.

- The Ceramics Science Program supports research on the development of predictive, quantitative theories of densification and microstructure development, the application of these theories to produce and evaluate advanced structural ceramics with improved high-temperature performance, and new understanding of fatigue and other failure mechanisms in ceramics.

- The Biomolecular Materials Program explores the application of recent advances in biochemistry, molecular biology, and bioorganic chemistry to the synthesis of novel materials. Emphasis is on the use of natural, genetically engineered and "created" enzymes, novel (bio)polymers, and self-assembling thin films to control surface properties and form the basis of sensors.

- The High- $T_{c}$ Superconductivity Program focuses on basic science, including theory, synthesis, and characterization of new materials; and on thin films and their applications, including devices such as SQUIDs and bolometers.

The Air Force Office of Scientific Research supports x-ray microscopy and high-brightness $\mathrm{x}$-ray studies. The Office of Naval Research supports studies of oxide scales on aluminides, quantum Monte Carlo calculations, thin-film superconductors, zinc cells, and microwave-radiation-stimulated release of drugs. The Navy also sponsors research on efficient lighting for ships and optical properties of the ocean.

The Advanced Research Projects Agency (ARPA) is expanding its support of research in $x$-ray lithography at the CXRO. The projects supported by ARPA are on the critical path for the development of EUV patterntransfer technology. The research is in response to unique opportunities for science and technology at the ALS. Funding for the design of an undulator beamline was received in FY 1991 through LBL's CXRO is also acquiring an 
advanced direct-write electron beam lithography system with ARPA funding. This e-beam writer, or "nanowriter," will be used to support $x$-ray lithography research through the fabrication of zone plates and x-ray masks. Funds for new instrumentation are expected to continue in FY 1995. LBL may also receive increased ARPA support for dual-use R\&D for magnetic materials microscopy and for molecular design.

ARPA funds the MAGIC gigabit testbed, a project to combine highspeed, wide-area-network technology, distributed image-storage systems, and high-speed graphics with aerial and satellite images, to present a user with a virtual reality that corresponds to what a real observer would see while traveling through the real terrain. $L B L$ is doing the research and development for the high-speed distributed-image server system that will supply the imagery to the terrain-visualization application providing the real-time view of the landscape.

\section{Technology Transfer Program Direction}

To support DOE's mission to increase the nation's technological competitiveness, LBL has strengthened its programs promoting technology transfer and science and engineering education. LBL has put in place numerous projects to help link its research and resources to private industry and to enhance its contributions to the nation's education system for the benefit of both teachers and students.

The new Technology Transfer Program strives to make technology transfer an integral part of all LBL programs. It identifies promising research early in the creative process and guides it through to its industrial application. The Technology Transfer Department has comprehensive responsibility for providing technology transfer services to the divisions, including work for others and user facilities agreements. The department works closely with the research divisions to select and promote technologies available for licensing or collaboration with industry. It negotiates intellectual property agreements and Cooperative Research and Development Agreements (CRADAs); manages the interface with licensees and CRADA participants; and oversees submission, approval, and tracking of sponsored research projects. The Technology Transfer Department also keeps researchers apprised of new opportunities and acts as an interface for DOE small business partnerships.

\section{Work for Others}

LBL is engaged in $\$ 41$ million worth of sponsored research agreements that are non-DOE funded. Examples include:

- $\$ 16$ million per year from the NIH to support various research projects in the Life Sciences Division

- $\$ 6.1$ million from Amgen to support the automation of a generation of sorted cDNA libraries

- $\$ 491,000$ from Loma Linda to transfer Bevalac technology to Loma Linda Medical Center

- $\$ 525,000$ from Pfizer Pharmaceuticals to support structural studies of the TCell Type-n potassium channel

- $\$ 941,000$ from NASA for a training grant in radiation health 
Technology Transfer Program

\begin{tabular}{|c|c|c|c|c|c|c|c|c|}
\hline Category & $\begin{array}{l}\text { FY } \\
1993\end{array}$ & $\begin{array}{l}\text { FY } \\
1994\end{array}$ & $\begin{array}{l}\text { FY } \\
1995^{a}\end{array}$ & $\begin{array}{l}\text { FY } \\
1996^{a}\end{array}$ & $\begin{array}{l}\text { FY } \\
1997^{a}\end{array}$ & $\begin{array}{l}\mathrm{FY} \\
1998^{\mathrm{a}}\end{array}$ & $\begin{array}{c}F Y \\
1999^{a}\end{array}$ & $\begin{array}{c}\mathrm{FY} \\
2000^{\mathrm{a}}\end{array}$ \\
\hline \multicolumn{9}{|l|}{ Activity } \\
\hline $\begin{array}{l}\text { Industry Cooperative } \\
\text { Agreements }\end{array}$ & 15 & 28 & 29 & 29 & 30 & 30 & 35 & 35 \\
\hline Agreement Value $(\$ M)^{b}$ & b 6.2 & 7 & 18 & 20 & 24 & 30 & 36 & 36 \\
\hline Personnel Exchanges & 6 & 6 & 7 & 7 & 7 & 7 & 7 & 7 \\
\hline \multicolumn{9}{|l|}{ Staffing } \\
\hline $\begin{array}{l}\text { Technology Transfer } \\
\text { Department }{ }^{c}\end{array}$ & 6 & 12 & 14 & 14 & 14 & 14 & 14 & 14 \\
\hline
\end{tabular}

aEstimated approved (CRADAs only).

bThis represents total dollars awarded by calendar year (vs. funding received annually).

Includes Office of Sponsored Research Administration, promotion, program management, and licensing efforts.

\section{Marketing/Outreach}

\section{Partnerships with Industry}

The outreach objective at $L B L$ is to increase the recognition of LBL as a major resource for new technologies and partnerships. To promote LBL's technologies, the Technology Transfer Department uses a broad range of communication tools, from press releases and brochures to exhibiting at trade shows. As a result of successful marketing efforts, LBL received over 1500 inquires from the private sector in FY 1993. Each inquiry receives personal, one-on-one contact by letter, by phone, or by visiting LBL facilities. An LBL inquiry database is maintained to track interactions. One example of a successful communications effort is the "New Technology Announcement." The attributes of a new invention available for licensing or collaborative partnership are summarized using attractive layouts that highlight potential product applications. The package is sent to the corporate executive in charge of company development, targeted through a hightechnology corporate database. LBL also distributes the announcements to emerging technology newsletters and releases them to trade journals and the general press.

LBL has instituted several outreach innovations within the past two years. One example is LBL's joining the information superhighway. A variety of documents that describe Lawrence Berkeley Laboratory, including LBL's Catalog of Research Abstracts, are now available to users of Gopher and the World Wide Web via the global Internet. Further plans include listing technologies available for licensing and collaboration. Individuals in the private sector (including industry) can access LBL from anywhere in the world through the World Wide Web at the following address: http:// www.lbl.gov/lbl.html. Gopher users can access LBL via the server at gopher.lbl.gov. LBL was accessed more than 13,400 times from April 10th through April 16th. We estimate up to 20,000 accesses per week within the next month. 
Because outreach involves Laboratory networking, internal programs will focus on the important opportunities to perform collaborative research with U.S. industry. One way in which this is being accomplished is through a technology transfer liaison, a senior division manager or technology transfer specialist who acts as a single point of contact within each of the 12 research divisions to help disseminate technology transfer opportunities, new funding sources, updates, technology inquiries, and requests.

\section{ER-LTT Program}

LBL's Energy Research Laboratory Technology Transfer (ER-LTT) Program has been established to support DOE's overall Technology Transfer Program, which aims to enhance U.S. industrial competitiveness through beneficial collaborations between the national ER laboratories and industry. The program goal is to derive maximum value for the American public from technology developed at LBL. Funding mechanisms include "Spin-Off" Cooperative Research and Development Agreements (CRADAs), technology maturation projects, and personnel exchanges. The program also supports major projects like the AMTEX collaboration and the Advanced Computational Technology Initiative, which begins in 1995. Over 60 ER-LTT projects have been funded with industrial partners such as Rockwell, Amgen, Motorola, Seagate, Chiron, AccSys Technology, Octree Corporation, Advanced Photonics, Mas Par, and Intel. Other partners include IBM, AMTEX, Siemens, Rhone Poulenc, Kaiser Foundation Hospital, Motorola, and Bay Technical Products. DOE funding to date totals over $\$ 13$ million, and combined industry and DOE commitments exceed $\$ 30$ million.

\section{Industrial Development Program}

The Industrial Development Program is an outreach of the Technology Transfer Department staffed with personnel who have scientific and industrial backgrounds. Internally, LBL's evaluation of new scientific and technological results is coupled to markets and specific industrial candidates. Expertise and strategies for presentation of LBL capabilities, including technology transfer mechanisms, are then presented to industry and/or local institutions jointly with the scientific team.

To maximize the effectiveness of the $\mathrm{LBL} /$ industry efforts, the resources of LBL should be focused where the greatest leverage is gained from a match between Laboratory talent and infrastructure, and industry needs. The Laboratory would benefit most from a focus on local industries.

For example, biotechnology is a well-matched industry. The biotechnology industry was born out of UC San Francisco, UC Berkeley, Stanford, and LBL. The greatest concentration of biotechnology companies in the U.S. is located in the Bay Area and in California. This high-growth industry is expected to create one-third of the new jobs in California $(200,000)$ over the next five years. The industry is already closely tied to existing scientific programs with LBL, including the Human Genome Center, the Materials Sciences Division, the Structural Biology Division, the Life Sciences Division, and the Advanced Light Source. These divisions and centers employ a substantial fraction of the scientific and technical staff of the Laboratory. This synergy of human resources, a growth industry, unique physical infrastructure, and an industry with positive growth potential can keep the U.S. at the top of the international biotechnology industry. LBL intends to contribute to the success of this exciting field. 


\section{Opportunities for Small Business}

LBL's Technology Transfer Department is the designated contact for small business outreach. Small businesses are a major source of job creation, economic growth, and technological innovation in our economy. Current technology policies create both an opportunity and an obligation for all Federal agencies to sharpen their strategic focus on American industrial competitiveness and job creation. One of DOE's highest priorities is to work with existing public- and private-sector networks for reaching small and historically underutilized businesses. In the last round of approved LBL/ ER-LTT CRADAs, $100 \%$ were with small companies. LBL is committed to helping small businesses leverage their R\&D resources.

Intellectual Property

In addition to increasing its outreach efforts and encouraging its scientists to become involved in technology transfer opportunities, LBL has developed a "Technology and Invention Inventory," which lists LBL technologies (including patentable inventions, public domain technologies, and copyrightable works) in database form. The database yields information regarding subject area, patent and licensing status, and scientists. Portfolio categories of technologies that interest U.S. companies include transportation technology, chemical processes, new materials, sensors and detectors, energy-efficiency technology, ion sources, new instrumentation, medical applications, software, biotechnology, and environmental technology. The Laboratory is taking steps to identify potential intellectual property early in the creation process, to encourage its development, and to ensure its protection through appropriate means. The technology transfer staff works closely with the LBL Patent Department to ensure the early identification of commercializable ideas.

\section{Patents and Licensing}

LBL seeks to patent and license its intellectual property to strengthen the value of its inventions, both for use and application by industry and to promote the research and technology transfer interests of the Laboratory and its research staff. LBL made 27 patent applications in FY 1994, and 9 patents were issued. LBL licensing activity also has increased significantly since the 1980s, when relatively few agreements were made during the decade.

Licensing income typically arises from three terms in the license agreement: an up-front, one-time license issue fee; minimum annual royalties; and sales royalties. Intellectual property from a research laboratory may require considerable development effort. Thus, sales royalties are not expected to begin until several years after the license is signed. Licensing and royalty income is distributed according to University of California policy and is consistent with patent law. Patent prosecution costs are first deducted and University policy allows the Laboratory to assign $15 \%$ for administrative costs for licensing effort and in maintaining the license. After the inventor receives a share, the remainder is available for laboratory research. Consistent with patent law, $75 \%$ of the royalty income that exceeds $5 \%$ of LBL's annual budget will be paid to the U.S. Treasury. LBL evaluates the allocation of intellectual property rights in technology transfer agreements. 
Intellectual Property Management

\begin{tabular}{lcccrr}
\hline Category & FY 1993 & FY 1994 & $\begin{array}{c}\text { FY 1995 } \\
\text { (est.) }\end{array}$ & $\begin{array}{c}\text { FY 1996 } \\
\text { (est.) }\end{array}$ & $\begin{array}{c}\text { FY } 1997 \\
\text { (est.) }\end{array}$ \\
\hline New Licenses (No.) & & & 20 & 25 & 25 \\
License Income (\$K) $^{\mathrm{b}}$ & 16 & 280 & 150 & 180 & 220 \\
Patent Applications & 344 & 27 & 30 & 33 & 34 \\
Patents Issued & 27 & 9 & 21 & 23 & 23 \\
Patent Staffing & 12 & 3 & 3 & & 4 \\
Intellectual Property & 2.5 & & & & \\
Use of Incomec Management & & & & & \\
\hline
\end{tabular}

ancludes options.

bCash in and estimated fair market value of noncash income.

cInvention \& ORTA Administration: $15 \%$ of gross income plus patenting costs

Scientific or Applied R\&D: $50 \%$ of net income

Awards \& Inventor Payments: $50 \%$ of net income

Education/Training: 0

Other (Specify): 0

Industry-Laboratory Cooperative Projects

LBL is engaged in $\$ 41$ million worth of industrially-sponsored research agreements that are non-DOE funded. This represents a $10 \%$ increase over 1993. The Laboratory has also initiated a number of technology research participation programs to improve the transfer of emerging energy technology to private industry. This includes forefront developments in advanced nanostructures fabrication and novel biomedical instrumentation. The Laboratory is also supporting new CRADA opportunities and initiating pilot projects to promote cooperative research opportunities within specific program areas. These projects have been successful in attracting U.S. corporate interest. Currently, LBL has 47 signed CRADAs with private industry. CRADAs and private industry WFOs brought approximately $\$ 17$ million into LBL in 1994.

CRADAs in progress include: the California Institute for Energy Efficiency, Dow Chemical, the U.S. Advanced Battery Consortium, and the New York State Energy Research and Development Authority.

Examples of some current industry-LBL cooperative projects are:

- LBL, EPRI, and NY State to Develop Energy Efficiency Database. Lawrence Berkeley Laboratory has signed Cooperative Research and Development Agreements with the New York State Energy Research and Development Authority (Albany, NY) and the Electric Power Research institute (Palo Alto, CA) to jointly develop a database on energy-efficiency programs (DEEP). Cofunding commitments have been obtained from the Bonneville Power Administration, the Rockefeller Family and Associates, and The Energy Foundation.

DEEP will provide unbiased and accurate information on the cost and performance of utility demand-side management programs, with the aim of defining successful program approaches that can be applied to a broad range of regions and utilities. The database will consist largely 
of North American programs. Information provided by DEEP will include program descriptions and key summary data on program costs, participation rates, total eligible customer base, energy and demand savings, cost-effectiveness, and evaluation methodologies. The database will include additional practical information, such as program contacts. The primary source of the data will be evaluation reports prepared by utilities and state and regional governmental organizations. The database will also offer periodic analyses and summaries of pertinent data to present the lessons learned from particular types of programs (e.g., lighting programs in commercial buildings or appliance rebate programs for energy-efficient refrigerators). DEEP will be updated with information from additional energyefficiency programs as the information becomes available.

- LBL and Rouge Steel Company Focus on Paintability. Lawrence Berkeley Laboratory is working with Rouge Steel Company under terms of a 3-year CRADA to improve electrogalvanized steel used in automobile manufacturing. They will optimize steel finish for painting while maintaining superior formability. By gaining a better understanding of the metallurgical and processing variables that control finish, this project will further strengthen the U.S. automotive industry's competitive position with foreign competitors.

Rouge Steel Company, located in Dearborn, Michigan, is the eighth largest integrated steel producer in the U.S. and provides sheet steel products to the automotive industry. 
LBL's strategic planning addresses the need to align its management and operational systems to support DOE national research programs and to achieve Vision 2000. A number of organizational systems have been identified as critical factors for measuring the Laboratory's performance. The previous section addressed the programmatic initiatives and research directions whose performance is essential for meeting the needs of LBL's customers_principally DOE, other federal agencies, industrial partners, and state and local agencies. This section describes the management and operational support systems that support LBL's programs and are a key to successful research management and institutional planning.

\section{HUMAN RESOURCES}

LBL's strategic plan supports a major initiative in empowering and respecting all employees. This effort undergirds and supports DOE's efforts for aligning human resources with agency priorities, including increased diversification of the workforce, organizations with streamlined management, the utilization of process improvement tools in systems review, and empowered employees with lessened regulation by oversight so that LBL and the Department can respond to today's technology-demanding economy.

An LBL Human Resources strategic planning effort involving Division Directors, LBL Center Heads, and Senior Administrative and Human Resources staff has resulted in the formation of a vision and mission for the Human Resources Department at Lawrence Berkeley Laboratory. This mission is to positively influence the organization's culture by offering quality programs and services to our customers and proactively supporting all organization members in establishing, maintaining, and promoting a 
team-oriented work environment within an atmosphere of mutual respect and trust.

Each individual is provided opportunities and is challenged to reach his or her highest potential, resulting in maximum productivity, creativity, and job satisfaction.

Our ultimate goal is a motivated, committed, and educated work team, ready to serve our partners, peers, and the community to achieve the objectives of the Laboratory.

The Human Resources Strategic Plan has resulted in the creation of a set of operating principles and expected outcomes concerning the delivery of services in the areas of compensation, employee relations, labor relations, benefits, training, and staffing. In each of these areas, we are challenged to deliver services that add value to our customers.

The principles that guide our performance require us to establish a consultative partnership with our clients. Our efforts will support institutional goals and directives that are situationally rather than structurally based. We need to be flexible and offer a market-based compensation program focusing on duties and responsibilities. We will deliver training that is skilled and behavioral and value based, and that incorporates the "Laboratory culture." We expect a bipartisan employee relations climate that balances management's perspective with the employee's perspective, and where our Employee Relations staff is valued for its objectivity and quality consulting.

LBL's initiative directly addresses the barriers to achieving human resources goals that DOE has identified with many departmental elements. These barriers include resource constraints, complex personnel systems, competition for diversity talent, and historic cultures that impede trust and delegation of responsibility. The principal elements of LBL's human resources planning effort are discussed below and are also summarized in Section III, "Laboratory Strategic Plan."

\section{Laboratory Personnel and Programs}

LBL's most valuable resource is its people-the scientists, engineers, and support staff who contribute their many diverse skills to advance the Laboratory's research programs. The Laboratory's scientific and engineering staff are known for a wide range of accomplishments and honors. Nine LBL scientists have become Nobel laureates, sixteen have won Lawrence Awards, and four have won Fermi Awards. Of its present staff, 55 have been elected to the National Academies of Sciences and Engineering. Much of this success is founded on the Laboratory's ability to create highly effective teams of scientists, engineers, technicians, and students-then to orchestrate their efforts to produce a rich yield of basic knowledge and applied technology. From scientific leadership to technical expertise to administrative support, all parts of the team are necessary if we are to succeed.

To reinforce our successes, the Outstanding Performance Award (OPA) Program has been developed to recognize and reward individual and team efforts that support the Laboratory's strategic plan. Examples of these achievements include areas such as technology transfer, total quality management, and workforce diversity.

A large part of the Laboratory's success is also due to the many graduate and undergraduate students who contribute their efforts each year, as well 
as the many senior staff scientists jointly appointed as faculty on the UC campuses, primarily at UC Berkeley. This relationship with UC provides a unique ability to interact with the broader university community, and helps to attract and retain a professional staff of high caliber. All of these factors contribute to LBL's mission to promote excellence in education and training, both for its own employees and for the greater scientific community. Our ultimate goal is to offer exceptional opportunities for professional growth, in an environment where achievement is recognized and rewarded at every level.

The Laboratory's Human Resources programs are administered in a manner consistent with the Prime Contract with the University of California and the Department of Energy. The Laboratory has adopted a compensation philosophy with the following objectives:

- To provide a level of compensation that, within available funds, attracts, motivates, and retains the quality work force necessary for the achievement of Laboratory goals.

- To recognize and reward performance and productivity while maintaining a competitive market position and providing internal Laboratory equity.

In its administering of the Human Resources programs, the Laboratory strives to deliver Human Resources systems in a cost-effective manner; develop and maintain work force excellence; strengthen the Laboratory's commitment to achieving work force diversity; and guide Human Resources programs and operations by quality principles in accordance with the Laboratory's quality strategies and initiatives.

Human Resources programs and initiatives that are planned for the next fiscal year include:

- Developing and maintaining an applicant pool that is diverse, current, and highly qualified.

- Streamlining the hiring and recruitment process, with more scientific and engineering activity occurring in the research divisions and more administrative hiring occurring centrally.

- Providing affirmative action guidelines and resources.

- Sponsoring campus recruitment and internship programs.

- Developing a strategic plan for LBL training requirements.

- Implementing a career development program and an integrated management program with defined skill sets for each management level.

- Implementing supervisory training programs that reinforce and support Laboratory quality initiatives.

- Developing a proactive role for Employee Relations and Labor Relations staff that balances employee concerns with management needs.

- Developing programs that effectively coordinate the Laboratory's employee resources.

- Developing and implementing alternative rewards programs, including nonmonetary rewards, spot awards, and employee-initiated awards. 
Laboratory Staff Composition (Full- and Part-Time Personnel-FY 1993)

\begin{tabular}{lccccc}
\hline Group & Doctoral & Master's & Bachelor & Other & Total \\
\hline Professional Staff & & & & & \\
Staff Scientists & 763 & 77 & 60 & 11 & 911 \\
& $(22.0)$ & $(2.2)$ & $(1.7)$ & $(0.3)$ & $(26.3)$ \\
Engineers & 126 & 108 & 100 & 14 & 348 \\
& $(3.6)$ & $(3.1)$ & $(2.9)$ & $(0.4)$ & $(10.0)$ \\
Management/Administrative & 33 & 97 & 214 & 306 & 650 \\
& $(1.0)$ & $(2.8)$ & $(6.2)$ & $(8.8)$ & $(18.8)$ \\
Support Staff & & & & & \\
Technicians & 11 & 91 & 209 & 645 & 956 \\
& $(0.3)$ & $(2.6)$ & $(6.0)$ & $(18.6)$ & $(27.6)$ \\
All Other & 0 & 107 & 341 & 150 & 598 \\
& $(0.0)$ & $(3.1)$ & $(9.8)$ & $(4.3)$ & $(17.3)$ \\
Total & 933 & 480 & 924 & 1126 & 3463 \\
& $(27.0)$ & $(13.9)$ & $(26.7)$ & $(32.5)$ & $(100.0)$ \\
\hline
\end{tabular}

\section{Training and Development Programs}

Through a combination of on-the-job training, in-house training programs, and attendance at programs/courses provided by non-Laboratory institutions, the technical skills of the Laboratory's employees are constantly upgraded to keep current with rapid technological advances. Communication and training are the keys to achieving our diversity goals. For any program to be successful, it must be integrated at all levels of authority and supervision. Therefore, a diversity training program is mandatory for all managers and supervisors. This program not only helps them to understand cultural differences and manage diversity, but also solicits their assistance in creating a diverse work force at the Laboratory. As a follow-up to the mandatory diversity training, we have also provided training for targeted groups of supervisors and employees in employment law. For supervisors, the focus is on ensuring that the work environment is in full compliance with employment law dictates. For employees, the objective is to assist them in understanding their rights and responsibilities. In addition, it is the Laboratory's policy that all managers and supervisors be held accountable for achieving diversity goals, and as such they are reviewed annually to evaluate their performance with regard to affirmative action and Equal Employment Opportunity (EEO) policies.

The development of training in leadership skills continues. The pilot program now includes approximately 200 managers, supervisors, Laboratory Heads, and Division Directors. We have now moved beyond the pilot and are developing a program for the scientific organizations. Ultimately, this training will be integrated into the Laboratory's required training program.

In addition, the Human Resources Department offers a range of management and supervisory development courses. One of these is the ongoing training program to assist managers and supervisors of disabled employees. Special events have been held to heighten the awareness of the Laboratory community regarding employment of the disabled, including workshops and demonstrations of equipment to assist disabled employees. The Vocational Rehabilitation Service is available to assist supervisors in making accommo- 
dations for employees and potential employees with disabilities. Other important Human Resources Department programs and activities include on-site and off-site programs to assist employees in developing their skills. Several of these courses emphasize communications, including The Pronounced Difference: Speech Evaluation Workshop for Non-native Speakers of English. In addition, the Laboratory has an education assistance and tuition reimbursement program to assist employees in obtaining advanced training and education through approved employee development plans.

In addition to providing training, the Laboratory maintains an Employee Assistance Program (EAP), which offers several employee programs to promote job retention, personal well-being, and effective job performance. These resources and programs are available through the Human Resources Department's Employee Relations and Labor Relations offices and the Health Services Department. The EAP includes on-site consulting services for emotional problems and substance abuse as well as referral services for off-site resources.

More than 30 development courses and seminars are offered on site each year. The Laboratory also provides support for off-site training and education, including baccalaureate and advanced degrees as well as other professional training credentials. Scheduled on-site training and programs are conducted in environmental safety and health and in management, personnel, computer, and workstation skills.

\section{Work Force Diversity}

One of the guiding principles of Vision 2000 is a reaffirmation of our commitment to work force diversity - to a cultural ethic that fully respects every individual. The Laboratory is committed to affirmative action and EEO in all aspects of employment. This means integrating diversity into its research culture and providing an environment that is accessible, equitable, and hospitable to all employees.

The Laboratory's commitment, coupled with the recognition of the changing dynamics of the work force and increasing competition among the scientific and technological industries, means that we must work harder to make Lawrence Berkeley Laboratory an employer of choice. To achieve this goal, the Laboratory has formed a Work Force Diversity Office and a Committee on Diversity as well as initiated a wide range of programs that will enhance our ability to attract and retain qualified individuals. We recognize that there is still much to be done to accomplish this goal. What follows is a sampling of our efforts.

\section{Affirmative Action Programs: Accomplishments in 1993}

While an affirmative action program is required by federal regulations, the Laboratory considers affirmative action as a mechanism to promote diversity and as an integral part of the Laboratory's institutional and planning processes. The following is a representation of several programmatic achievements that occurred in 1993:

New Training Efforts. The Laboratory introduced two new training programs. Managers and supervisors in the Administration and Operations Divisions participated in the field testing of the Zenger-Miller "FrontLine Leadership" program and in a workshop entitled "Managing Within the Law." A similar workshop, "Employee Rights \& Responsibilities: Knowing the Law," is offered to employees. These training efforts 
demonstrate the Laboratory's renewed commitment to strengthening supervisory skills and addressing employee expectations and responsibilities in the workplace.

Outstanding Performance Awards. In October, the Laboratory sponsored its second annual Outstanding Performance Award program. The criteria for selection included contribution to outstanding techniques or procedures; technical innovation or notable accomplishment in improving environmental, health, or safety conditions at LBL; or demonstrated leadership and success in employee development, affirmative action, diversified work force, and community relations. The awards ranged from $\$ 1,000$ to $\$ 5,000$.

Career Development Initiatives. The Employee Training and Development Unit in the Human Resources Department developed and piloted a new department-based career development training program. Unit staff met with supervisors and managers in the Financial Management Department to discuss their roles as "career coaches." They then conducted employee training sessions that guided them through a skill assessment and interest and values clarification process. Employees were invited to participate in follow-up activities, which included group sessions and one-on-one career counseling. The Unit expects to offer the program on a broader basis in 1994 .

Community Relations Office. The Community Relations Office, now in its second year, initiated several new outreach efforts, including participation in Berkeley Chamber of Commerce Trade Fairs; participation in the city's celebration of Earth Week; and development of a Laboratorywide Outreach Network, which is a group of employees representing offices with outreach functions.

RESUMIX - Automated Résumé Screening to Promote Diverse Applicant Pools. The Human Resources Department purchased a computerized résumé scanner that allows the Laboratory to access thousands of résumés on file in a matter of minutes. This technology has facilitated efforts to follow through on contacts with individuals from protected classes interested in employment at the Laboratory. As a follow-up to outreach recruitment efforts, collected résumés are now placed into the system. Whenever a vacancy occurs, the system can identify all affirmative action candidates who appear to be likely candidates for the position. This technology should help the Laboratory develop more diverse applicant pools.

Management Skills Assessment Program. After a lapse of several years, the Laboratory renewed its participation in the Management Skills Assessment Program, an affirmative action training program administered by the University of California.

Easy Access to Current Job Opportunities (CJO) Bulletin. The CJO Bulletin is now available to both internal and external callers through a telephone job line accessed at (510) 486-4266. Additionally, the document is available electronically through computerized bulletin boards on the Internet (via Gopher and the World Wide Web), which can be accessed by thousands of users worldwide.

A Fine Line. The Laboratory, in cooperation with the Human Resources Department at Los Alamos National Laboratory, produced a new 
publication entitled A Fine Line: How to Recognize and Deal with Sexual Harassment in the Workplace. This 49-page booklet is used in related informal and formal training settings.

Child Care Center Working Group. The Director's Office has worked on developing a feasibility study for a day care center at the Laboratory. The Deputy Director convened a Child Care Center Working Group, conducted a Laboratory-wide survey to evaluate employee interest in a center, and is preparing a business plan for the center. Although a final decision has not been made, the year's efforts have moved the Laboratory forward in laying the foundation necessary to make a sound decision about the center.

Employee Associations. In 1994, the Women's Science and Engineering Group continued to meet to share research as well as mutual areas of professional concern. In response to strong employee interest, several employee groups met to develop employee associations aimed at supporting diversity at the Laboratory. The African-American Employee Association received recognition from the Laboratory Director and elected its first officers. The Association's activities included a formal induction ceremony and reception for newly elected officers, a picnic to welcome summer students participating in programs sponsored by the Center for Science and Engineering Education, and a canned food basket drive in cooperation with a local nonprofit organization for Thanksgiving. The Lesbian, Gay \& Bisexual Association was formed in March 1994, and in May 1994, the Latinos and Native Americans were also formally recognized.

\section{Affirmative Action 1994}

\section{Laboratory-wide Activities}

All Laboratory Divisions will continue to carry out a variety of baseline and ongoing development activities aimed at obtaining diverse applicant pools and developing and retaining employees. These activities comprise a program that has expanded steadily over the last three years. The following is a synopsis of these cross-Division activities. Specific actions initiated and carried out by particular divisions are included in later Division summaries.

Basic equal opportunity/affirmative action efforts have been incorporated into standard Division procedures such as the inclusion of EEO and affirmative action responsibilities in supervisors' performance reviews, development of recruitment plans when vacancies exist, equity review of salary actions, and participation in related training programs.

The Laboratory will continue to support development efforts by facilitating participation in training, through the tuition reimbursement program and on-the-job training. Formal programs will continue to be promoted, such as the UC Postdoctoral Program, the Management Skills Assessment Program, and the Leadership Education for Asian Pacifics (LEAP) Program.

Divisions will continue to participate in the Diversity Committee by sending representatives to discuss related issues and action-oriented planning. The Committee makes recommendations to the Director about ways to enhance the LBL work environment and accommodate the diverse needs of its employees. 


\section{Short-Range Plans}

LBL is committed to the following goals:

- An annual reduction of outstanding affirmative action goals based on opportunities, i.e., personnel "actions" that move an individual into a job group via promotion or transfer, or by the hiring of a new employee.

- Continued targeting of goals for placement of minorities and women at mid- and upper-management levels until these groups are better represented within all job areas at these management levels.

- An assessment of supervisors' affirmative action/EEO contributions in their annual performance reviews.

- As vacancies appear, LBL will continue to target openings to obtain representation of diverse ethnic groups and of women throughout the Laboratory. A special emphasis is being placed on all professional and technical positions so that we may attain our affirmative action goals and diversity in the Laboratory work force.

- A new focus on the recruitment and hiring of disabled persons will be made.

- The Work Force Diversity Office will continue to implement the recommendations contained in the Laboratory Committee on Diversity Report, as approved by the Laboratory Director.

- Establishing and continuing support for the Work Force Diversity Office, fully staffed, with strong administrative support, to fully implement and support the Affirmative Action Program while maintaining records and statistics regarding affirmative action activities.

- The Work Force Diversity Office will develop newly designed affirmative action reports for Division Directors to assist Department Heads in managing their affirmative action responsibilities and to assess how well Divisions are using available opportunities to improve and meet their work force affirmative action goals.

- Line management will be targeted for greater participation in affirmative action recruitment for vacancies as they occur in the Divisions. Managers will attend job fairs and related events and speak directly with qualified individuals interested in working in their specific area of operations.

- A Recruitment Resource Guide, under development, will be available for circulation in FY 1995. It will serve as a resource to supervisors and managers in their recruitment planning for job vacancies.

- Implementation of a Laboratory mentoring program (pilot) in the Engineering Division.

- Implementation of Laboratory-wide diversity training for new supervisors and managers.

- The Work Force Diversity Office, in conjunction with the Laboratory's employee associations, will sponsor multicultural diversity awareness/ education events and programs.

\section{Long-Range Plans}

- New priority will be placed on targeting underrepresented groups so as to place more individuals from these groups in management positions. Community outreach activities, refined and selective recruitment activities, and affirmative action networking will be utilized for this purpose. 
- Monitoring activities will be expanded and/or refined to include:

Merit increases

Upward mobility

Promotions

Transfers

Special recruitment

Reorganization

Classification

Training and staff development

Discipline

Retention

Issues related to labor relations

- Work force statistical goals will be attained through the use of training programs and employment pools, and through the targeting of management-level positions. The Laboratory will direct proactive efforts toward reduction and/or attainment of affirmative action goals in job groups and/or classifications.

\section{Affirmative Action Plans}

On an annual basis, the Laboratory prepares two Affirmative Action Plans, which are approved by the University of California and provided to the U.S. Department of Energy. The Equal Opportunity Administrator is responsible for the audit and implementation of the Laboratory's Affirmative Action Program. The Affirmative Action Plans allow the Laboratory to establish and audit its affirmative action and EEO activities and its performance. The first Plan focuses on women and underrepresented groups, and the second Plan focuses on Vietnam-era veterans and persons with disabilities. These Plans are distributed to each of the Laboratory's 13 Divisions, senior-level management offices, and the Laboratory's five on-site libraries. Copies are available to employees, applicants, and compliance agencies upon request through the Work Force Diversity Office. The Plans provide the Laboratory with an opportunity to discuss analyses of the Laboratory's underutilization and division-based adverse impact as well as Laboratory-wide and division-based affirmative action efforts of the preceding year. On an annual basis, the Equal Opportunity Administrator meets with the Laboratory Director, Deputy Laboratory Director, Associate Laboratory Directors, and each Division Director to discuss the Plans in detail.

The following two tables are excerpted from the Laboratory's Report of Employment and Labor Turnover for fiscal years 1988 and 1993. The data source for each report is based on the preceding fiscal year (October 1st through September 30th). The two tables show Laboratory populations for the 1988 and 1993 fiscal years and provide breakdowns by federal occupation category and by race. Population figures are based on career-status employees. Despite the shrinking pool (Ph.D. level) of available women and underrepresented groups, the Laboratory population has remained relatively constant during this period. To increase the recruitment pool of qualified individuals, the Work Force Diversity Office will undertake various programs and initiatives, such as those mentioned above, that focus on staff career development as well as outreach recruitment to offset the dearth of women and underrepresented groups in the pipeline. 
Lawrence Berkeley Laboratory 1988

\begin{tabular}{|c|c|c|c|c|c|c|c|c|}
\hline \multirow{3}{*}{$\begin{array}{l}\text { Federal Occupational Category } \\
\text { Gender }\end{array}$} & & & \multirow{3}{*}{$\frac{\text { Total }}{\text { White }}$} & \multicolumn{5}{|c|}{ Minority } \\
\hline & \multicolumn{2}{|c|}{ Total } & & \multirow{2}{*}{$\begin{array}{l}\text { Total } \\
M \& F\end{array}$} & \multirow{2}{*}{$\frac{\text { Black }}{\mathrm{M} \& \mathrm{~F}}$} & \multirow{2}{*}{$\frac{\text { Hispanic }}{M \& F}$} & \multirow{2}{*}{$\frac{\text { Asian }}{M \& F}$} & \multirow{2}{*}{$\frac{\text { Nat. Am }}{\text { M\&F }}$} \\
\hline & Male & Female & & & & & & \\
\hline OFFICIALS \& MANAGERS & $\begin{array}{c}92 \\
81.42 \%\end{array}$ & $\begin{array}{c}21 \\
18.58 \%\end{array}$ & $\begin{array}{c}102 \\
90.27 \%\end{array}$ & $\begin{array}{c}11 \\
9.73 \%\end{array}$ & $\begin{array}{c}7 \\
6.19 \%\end{array}$ & $\begin{array}{c}3 \\
2.65 \%\end{array}$ & $\begin{array}{c}1 \\
0.88 \%\end{array}$ & $\begin{array}{c}0 \\
0.00 \%\end{array}$ \\
\hline $\begin{array}{l}\text { PROFESSIONALS } \\
\text { Scientists \& Engineers }\end{array}$ & $\begin{array}{c}677 \\
84.52 \%\end{array}$ & $\begin{array}{c}124 \\
15.48 \%\end{array}$ & $\begin{array}{c}674 \\
84.14 \%\end{array}$ & $\begin{array}{c}127 \\
15.86 \%\end{array}$ & $\begin{array}{c}12 \\
1.50 \%\end{array}$ & $\begin{array}{c}11 \\
1.37 \%\end{array}$ & $\begin{array}{c}103 \\
12.86 \%\end{array}$ & $\begin{array}{c}1 \\
0.12 \%\end{array}$ \\
\hline Management \& Administrative & $\begin{array}{c}61 \\
32.80 \%\end{array}$ & $\begin{array}{c}125 \\
67.20 \%\end{array}$ & $\begin{array}{c}136 \\
73.12 \%\end{array}$ & $\begin{array}{c}50 \\
26.88 \%\end{array}$ & $\begin{array}{c}18 \\
9.68 \%\end{array}$ & $\begin{array}{c}10 \\
5.38 \%\end{array}$ & $\begin{array}{c}22 \\
11.83 \%\end{array}$ & $\begin{array}{c}0 \\
0.00 \%\end{array}$ \\
\hline TECHNICIANS & $\begin{array}{c}440 \\
86.61 \%\end{array}$ & $\begin{array}{c}68 \\
13.39 \%\end{array}$ & $\begin{array}{c}391 \\
76.97 \%\end{array}$ & $\begin{array}{c}117 \\
23.03 \%\end{array}$ & $\begin{array}{c}35 \\
6.89 \%\end{array}$ & $\begin{array}{c}25 \\
4.92 \%\end{array}$ & $\begin{array}{c}56 \\
11.02 \%\end{array}$ & $\begin{array}{c}1 \\
0.20 \%\end{array}$ \\
\hline $\begin{array}{l}\text { All Other (Crafts, Laborers, Service } \\
\text { Workers, Office, and Clericals) }\end{array}$ & $\begin{array}{c}303 \\
52.97 \%\end{array}$ & $\begin{array}{c}269 \\
47.03 \%\end{array}$ & $\begin{array}{c}353 \\
61.71 \%\end{array}$ & $\begin{array}{c}219 \\
38.29 \%\end{array}$ & $\begin{array}{c}116 \\
20.28 \%\end{array}$ & $\begin{array}{c}58 \\
10.14 \%\end{array}$ & $\begin{array}{c}39 \\
6.82 \%\end{array}$ & $\begin{array}{c}6 \\
1.05 \%\end{array}$ \\
\hline Total All Categories & $\begin{array}{c}1573 \\
72.16 \%\end{array}$ & $\begin{array}{c}607 \\
27.84 \%\end{array}$ & $\begin{array}{c}1656 \\
75.96 \%\end{array}$ & $\begin{array}{c}524 \\
24.04 \%\end{array}$ & $\begin{array}{c}188 \\
8.62 \%\end{array}$ & $\begin{array}{c}107 \\
4.91 \%\end{array}$ & $\begin{array}{c}221 \\
10.14 \%\end{array}$ & $\begin{array}{c}8 \\
0.37 \%\end{array}$ \\
\hline
\end{tabular}

Source: 1989 LBL Affirmative Action Plan. Figures are based on fiscal year 1988 (10/1/87-9/30/88). 
Lawrence Berkeley Laboratory 1993

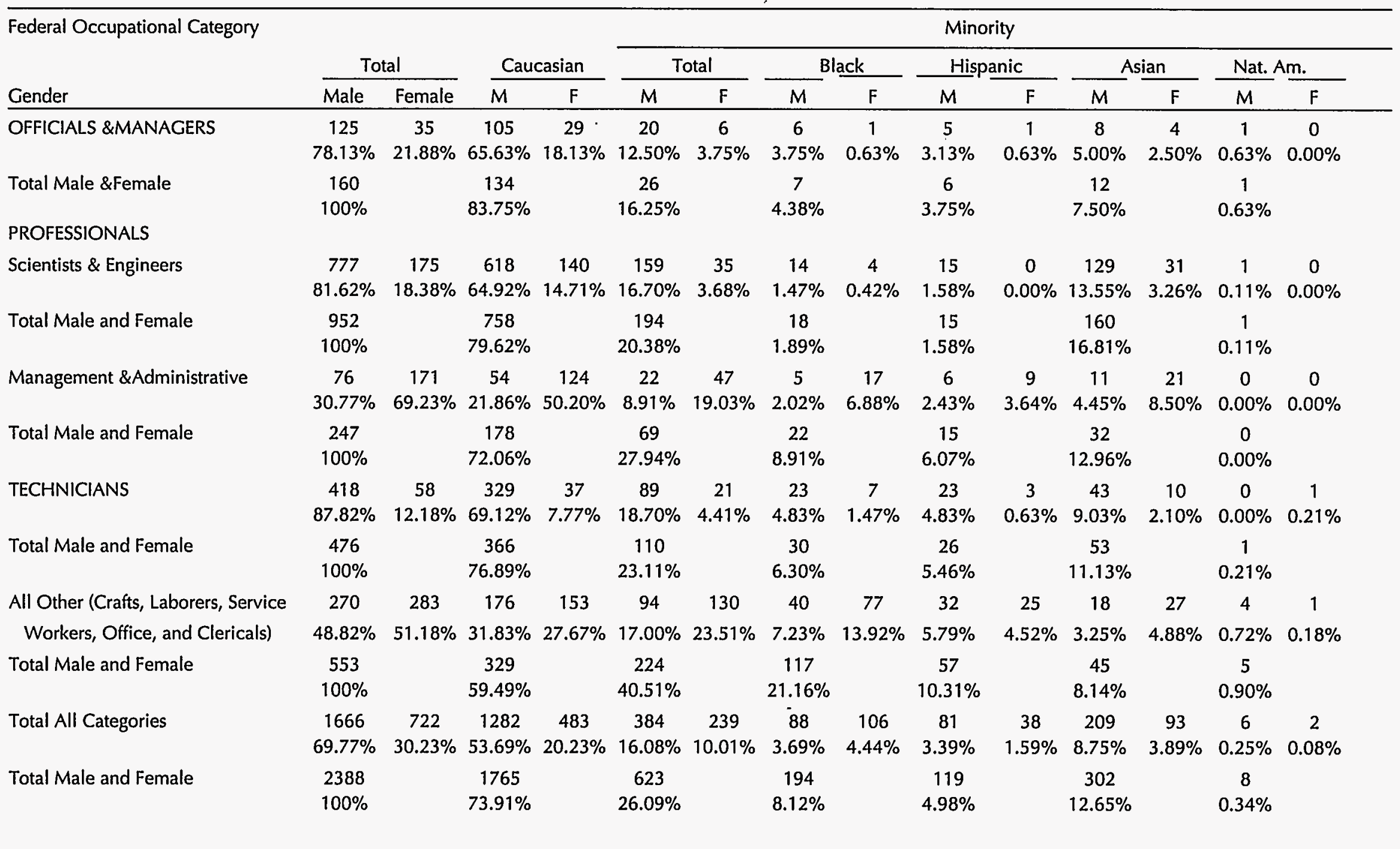




\section{... ENVIRONMENT, SAFETY, AND HEALTH}

LBL's environment, safety, and health programs, which are integral to program performance, fully support DOE's Critical Success Factor Strategies for ensuring the safety and health of workers and the protection and restoration of the environment. Excellence and timely implementation of environmental, safety, and health activities are critical to the success of each of LBL's-and DOE's - core business areas. LBL strongly endorses DOE's vision that the hallmark and highest priority of all our activities is daily excellence in the protection of the worker, the public, and the environment. LBL's EH\&S programs correspond to and cooperate with all four of the DOE goals in support of this vision. Our entire program overlaps DOE's Goal 1. Performance measures corresponding to Goal 2 are incorporated into the DOE-UC contract for the management of LBL. EH\&S priorities are set and followed in accordance with DOE's EH\&S Management Plan and the DOE Environmental Restoration and Waste Management Five-Year Plan to satisfy the requirements of Goal 3. Also, LBL is striving diligently to demonstrate respectable performance related to environmental protection and worker/ public safety, as envisioned by Goal 4 . The programs described below are fundamental to the attainment of this vision and represent the performance commitment of LBL employees to trust, communications, and continuous improvement in all LBL activities.

\section{ES\&H Goals and Objectives}

It is the policy of the Lawrence Berkeley Laboratory to integrate environment, safety, and health (ES\&H) performance into all of its operations to ensure employee and public safety and the protection of the environment. The Laboratory has developed institutional ES\&H goals to guide its integrated ES\&H program. The ES\&H goals are the following:

- LBL will provide employees with a safe workplace.

- LBL will design and operate facilities and research activities to minimize adverse impact on public health and the environment.

- LBL will produce and use only materials that can be disposed of safely and will minimize waste.

- LBL will promptly communicate the known hazards of our activities and the related methods necessary for safety and health protection.

- LBL will use available technology, engineered safeguards, and responsible science to mitigate all significant risks arising from its research and related activities.

The objectives of ES\&H in the conduct of research activities are to ensure the integrity of human health and safety and the environment in which we operate, to manage resources with a value-added perspective, to maintain a capability that is not currently supported by other Laboratory programs, to provide opportunities for staff development, to build new competencies that could prove useful to future Laboratory and DOE ES\&H programs, and to support the Laboratory's technology transfer mission.

Underlying these goals is a commitment to ES\&H performance through 
quality management of the Laboratory's programs and in its conduct of operations. LBL's current efforts respond to the management initiatives of the DOE Office of Energy Research (ER), and directly to the Corrective Action Plan for the Tiger Team Assessment and ER review. The Laboratory has also developed ES\&H management and is developing implementation programs for a DOE Environmental Restoration and Waste Management Five-Year Plan. These efforts include a renewed commitment to ES\&H through self-assessment and an effective Corrective Action Plan.

The Laboratory's ES\&H Performance Measures are utilized to improve performance and institute a more quantitative framework for LBL's ES\&H trends and activities. For employee health and safety, representative measures include those that document occupational radiation doses and accidental frequency and severity rates (expressed as cases or days lost per 200,000 hours worked). In accordance with the requirements of Contract 98, LBL has embarked on a course of continuous improvement in ES\&H performance over prior years.

Environmental Performance Measures include public radiation doses from LBL operations, waste minimization, and the Toxics Release Inventory. The goal of waste minimization is to manage waste disposal more effectively and efficiently, including significantly reducing the total amount of hazardous wastes generated when compared to prior years. Waste minimization indicators include the percent of Laboratory office waste recycled, and the total number of waste streams recycled. Performance Measures include . those that count the number of waste pick-up requisitions rejected because waste is nonhazardous (waste minimization), the number of complaints received by a group leader (waste management operations), and the number of requisitions received and processed with average turn-around time for each requisition (waste management operations). In addition, the Laboratory is establishing procedures to assure that there will be no excess discharge of heavy metals and toxic chemicals into the sewer system. The goal is to keep all discharges below established standards.

\section{Current Conditions}

\section{Programmatic Directions and Potential Hazards}

As indicated in Section IV, LBL's scientific and technical programs primarily support DOE's Office of Energy Research (62\%). The multiprogram character includes programs in Basic Energy Sciences, Nuclear Physics, High Energy Physics, and Health and Environmental Research. Energy Efficiency and Renewable Energy (6\%) supports studies in building energy conservation, energy storage, and solar and geothermal energy. Other DOEsponsored programs $(16 \%)$ include research supported by Civilian Radioactive Waste Management, and Fossil Energy. Work for Other Agencies and Institutions (16\%) is primarily for the National Institutes of Health (NIH), Department of Defense (DOD), states, and private industry.

LBL's potential ES\&H risks are characteristic of accelerator operations, shops, and a diversity of laboratories for chemical, biological, materials science, and technology development, as well as other facility support operations. Hazards arise from both radiological and nonradiological activities. Radiation protection for workers and the public is required for accelerators, $x$-ray units, sealed sources, and radioisotope use. The nonradiological hazards include electrical systems, sources of ignition and 
combustible materials, rotating and reciprocating machinery, hoisting and rigging operations, lasers, chemicals, biohazards, moving vehicles, construction activities, and natural phenomena such as storms and earthquakes.

LBL monitors the levels of chemicals, biohazards, and radioactivity discharged from operations at the Laboratory, and evaluates their impact on the environment and to the public health. Radiological operations include particle accelerators, gamma irradiators, laboratories conducting research using radionuclides and radiopharmaceuticals, and the National Tritium Labeling Facility. Nonradiological sources and discharges include chemicals from research operations, fabrication shops, automotive shops, paint shops, water treatment facilities, and the Hazardous Waste Handling Facility. Current trends in Laboratory activity indicate the following environmental and safety hazards that must be mitigated:

- Chemistry and Materials Research. Programs in materials and chemical research, including new hazardous materials management requirements, create demands on many ES\&H programs. Examples include managing chemical acquisition and inventories, chemical monitoring systems, fume hood monitoring, laser safety training, respiratory protection prograrns, and hazards communications. The ALS, a controlled facility, expands the use of $x$-rays on site, and is operated with complete ES\&H systems and management protocols in place.

- Biological Research. LBL biological research programs have grown recently. LBL's biological hazards control program continues to be expanded. Biological research programs often utilize radiolabeled materials, and the delivery of low-level radioisotopes has increased in the last few years. The radioactive and mixed wastes derived from these operations present a special challenge because of the restrictions on their disposal.

- Radiobiology Experimental Programs. LBL radiological research at the Bevalac has closed, but the use of isotopes is expected to continue in many applications, including the new Biomedical Isotope Facility.

- Construction. LBL's construction activity has increased during the past decade, resulting in potential additional accident risk. ES\&H oversight for construction contractors and tradespersons has become increasingly important, including, but not limited to, the need to ensure the stability of excavations and management of drainage systems.

- Nuclear Physics and Nuclear Chemistry. The nuclear physics experimental programs at the Bevalac were curtailed in FY 1993. However, the 88-Inch Cyclotron has expanded research with the Gammasphere detector and supports a more diversified scientific program. In addition, planning is under way for an IsoSpin Laboratory that would utilize radioactive isotopes.

Increased resources have been provided to serve research program areas that have potential hazards, such as those employing toxic chemicals, lasers, new $\mathrm{x}$-ray sources and beamlines, and radiolabeled compounds. Space for staff and equipment must be provided to meet service demands and logistics needs. Reporting functions have been formalized to optimize use of staff. Automated systems to track chemicals from procurement to disposal and to automate all monitoring and analytical activities are under development. LBL is committed to meeting all regulatory requirements in a cost-effective, 
risk-based fashion that deals with real risks and environmental concerns as the top priority.

\section{Activities and Accomplishments}

\section{Environment Department}

During the past year, LBL's Hazardous Waste Handling Facility (HWHF) received full authorization to ship all kinds of waste (hazardous, radioactive, and mixed radioactive and hazardous) from the HWHF to off-site hazardous-waste facilities, including the Westinghouse Hanford Company's Hanford, WA radioactive-waste site. This authorization ended a two-year moratorium on shipments of these wastes from LBL, established as a result of an audit by the Westinghouse Hanford Corporation and the DOE "Tiger Team" visit of January 1991. The moratorium was declared on shipment of waste from LBL until the waste management work methods and documentation could be upgraded to ensure that all LBL waste was characterized and packaged correctly. EH\&S undertook a major upgrading of waste management documentation, procedures, and work methods, as well as a major upgrade of the HWHF itself. Waste shipments have now resumed, and the backlog of "legacy" waste (including mixed waste) built up during the moratorium has been eliminated.

\section{Health Department}

EH\&S's Radiation Assessment Group undertook a major program upgrade this past year. The Group completed and issued the Radiological Control Manual, which was required by both federal law and DOE orders. The Group also began documenting Lab-wide radioisotope use via a newly developed Project Review and Radioisotope Permitting Program. Under this Program, all LBL radioisotope users must complete a Radioisotope Project Review Form, which looks at all health and safety aspects of the proposed project related to radioisotope usage.

\section{Safety Department}

The LBL Emergency Command Center (ECC) was upgraded significantly during the past year. This upgrade was a long-needed project that received a boost after the disastrous 1991 Oakland Hills fire, which came within a few miles of LBL. The upgraded ECC features a dedicated line to the Fire Department Dispatch Center, located in the same building. Additional telephones were installed, and new maps, checklists, and administrative supplies were also provided. LBL also formed an Automatic Aid Agreement with the City of Berkeley Fire Department. The agreement allows the LBL Fire Department to respond to fires in the adjoining Oakland and Berkeley hill areas. The agreement should improve fire safety greatly in the hill areas adjoining LBL.

\section{ES\&H Plans and Initiatives}

To promote integration of strategic and program planning, the Laboratory maintains a Comprehensive Planning Calendar that defines annual planning requirements and provides a schedule that identifies necessary information exchange and preparation responsibilities. This process defines the responsibilities and information requirements intended to promote the 
integration of environmental, safety, and health concerns into institutional and program planning elements. Examples of planning products include those for program plans and for ES\&H support functions:

- ES\&H planning and initiatives. The EH\&S Division has realigned its mission to align with the broader mission of the Laboratory. To this end, the Division effectively manages environment, health, and safety in a way that minimizes interference with new or ongoing scientific research. Creating value for the economy and contributing to the community through partnerships with industry is another part of the Laboratory's mission. The EH\&S Division is developing creative solutions for environment, safety, and health problems that can be transferred into the private sector for broader application.

- ES\&H management and operations planning. LBL manages and coordinates its ES\&H programs through strategic plans that define activities, source needs, staffing, and regulating responsibilities. These plans include, as examples, the Tiger Team Assessment Corrective Action Plan, the ES\&H Management Plan, the Self-Assessment Implementation Plan, NEPA programs planning, and waste minimization plans (see below). Other examples are the Environmental Protection Implementation Plan, the Environmental Monitoring Plan, and the Groundwater Protection Management Plan.

- LBL emergency preparedness and response planning. This includes maintaining and updating a Master Emergency Plan, building and facility emergency plans, and individual equipment emergency plans. $\mathrm{LBL}$ is revising and strengthening these plans and implementing programs to ensure that resources and trained staff are available to address all credible emergencies.

- Research program planning. As an example of this program planning, early in the formulation of the Induction Linac Systems Experiments (see Section IV), the underlying ES\&H criteria for this facility were established, as documented in a Conceptual Design Report. The safety systems and procedures for this facility are being designed to meet all standards for the expected occupancy. Safety management operations have been integrated with program plans and are included in cost estimates. The National Environmental Policy Act (NEPA) document preparation schedule and the requirements for Operational Safety Procedures were also planned from the outset. The Human Genome Laboratory underwent a similar review and documentation process.

- Site development planning. The Site Development Plan integrates ES\&H objectives and needs in all facilities-related programmatic building support projects. Site planning goals, existing conditions, and planning analysis integrate ES\&H planning information, including NEPA and CEQA requirements. Specific references are made to environmental monitoring, environmental impact studies, and the health and safety of facilities. To provide adequate ES\&H support facilities, two general-purpose building initiatives are proposed within the period covered by this plan. Current Laboratory support service 
facilities are inadequate and inefficient because of obsolete design and substandard construction.

\section{Corrective Action Plan}

LBL's Tiger Team Assessment Corrective Action Plan, completed in September 1991, addresses the findings and concerns of the Tiger Team as well as the 1989 Technical Safety Appraisal. The Laboratory and DOE/OAK developed 409 tasks with subsidiary milestones to correct the findings and concerns and eliminate the underlying root causes. These root causes addressed the need for:

- Greater formality of operations and effective verification of the meeting of environmental, safety, and health requirements.

- More effectively addressing the demands of environment, safety, and health requirements and the urgency of incorporating these demands into LBL operations.

- Providing DOE program direction and oversight that place adequate emphasis on environmental, safety, and health requirements.

LBL has made significant progress on the Corrective Action Plan. A majority of the findings have been completed with the completion of $95 \%$ of the corrective actions. All Category II OSHA findings have been corrected or reduced to lower levels. LBL is continuing to allocate significant resources to the Corrective Action Plan effort. Complete implementation of the Corrective Action Plan also requires the support of the Office of Energy Research and Office of Environmental Restoration and Waste Management, as well as the providing of additional resources for LBL. In February 1993, a

ES\&H Program and Corrective Action Plan Resource Requirements (\$M)

\begin{tabular}{lcccccccc}
\hline Category & 1993 & 1994 & 1995 & 1996 & 1997 & 1998 & 1999 & 2000 \\
\hline Corrective Action Plan & & & & & & & & \\
Office of Energy Research & & & & & & & & \\
$\quad$ Operating & 0.7 & 0.7 & 0.0 & 0.0 & 0.0 & 0.0 & 0.0 & 0.0 \\
GPP & 1.7 & 0.1 & 0.0 & 0.0 & 0.0 & 0.0 & 0.0 & 0.0 \\
GPE & 0.0 & 0.0 & 0.0 & 0.0 & 0.0 & 0.0 & 0.0 & 0.0 \\
$\quad$ MEL-FS & 2.0 & 1.7 & 2.0 & 1.1 & 0.0 & 0.0 & 0.0 & 0.0 \\
ERWM & 1.1 & 1.1 & 0.1 & 0.0 & 0.0 & 0.0 & 0.0 & 0.0 \\
Laboratory Overhead & 1.5 & 1.1 & 0.0 & 0.0 & 0.0 & 0.0 & 0.0 & 0.0 \\
$\quad$ Total Corrective & & & & & & & & \\
$\quad$ Action Plan & 7.0 & 4.7 & 2.1 & 1.1 & 0.0 & 0.0 & 0.0 & 0.0 \\
\hline
\end{tabular}




\section{Environment, Safety, and Health Management Plan}

The Laboratory has developed and utilizes a prioritized Management Plan for Environment, Safety \& Health Activities that includes the existing core program of environment, safety, and health activities, additional basic support, and specific projects to fully meet all LBL and DOE environmental, safety, and health goals. LBL contributed to the development of the ER prioritization system to rank activities based on quantitative risk reduction criteria for the purpose of allocating funds.

In response to the requirements of the Corrective Action Plan, and to emphasize safety and health issues, LBL has significantly increased its spending on core environment, health, and safety programs since 1991. Conscientious application of prioritization methodology to these issues will allow the Laboratory and DOE to allocate appropriate funding each year, and to assure that serious and urgent issues are addressed appropriately in light of resource limitations.

Management Plan Projections

\begin{tabular}{lrrrrrrrr}
\hline Category & 1993 & 1994 & 1995 & 1996 & 1997 & 1998 & 1999 & 2000 \\
\hline Operating & & & & & & & & \\
Core & 10.4 & 10.3 & 10.3 & 10.6 & 10.9 & 11.3 & 11.9 & 11.9 \\
Improvements & 8.4 & 10.9 & 15.1 & 22.0 & 22.0 & 21.1 & 20.0 & 20.5 \\
Total Op. & 18.8 & 21.2 & 25.4 & 32.6 & 32.9 & 32.4 & 31.9 & 32.4 \\
Gap. Equipm't & & & & 0.5 & 0.1 & 0.1 & 0.1 & 0.1 \\
GPP & & & & 3.4 & 0.2 & 0.8 & 0.1 & 0.1 \\
Line Item & & & & 9.0 & 14.7 & 44.0 & 18.0 & 11.0 \\
\hline
\end{tabular}

\section{Waste Minimization Plan}

LBL's waste minimization program is an organized, comprehensive, and continual effort to systematically reduce hazardous, radioactive, and mixed waste generation. The DOE Waste Minimization and Pollution Prevention Awareness Program and California Senate Bill 14 (HazWaste Source Reduction and Management Review) are designed to eliminate or minimize pollutant releases to all environmental media from all areas of the site's operations. These efforts offer increased protection of public health and the environment. They will yield the following additional benefits:

- Reduction of waste management and compliance costs.

- Reduction of resource usage.

- Reduction or elimination of inventories and releases of hazardous chemicals.

- Reduction or elimination of civil and criminal liabilities under environmental laws. 
The program reflects the goals and policies for waste minimization for LBL and represents an ongoing effort to make waste minimization/pollution prevention part of the site's operating philosophy. LBL's overall efforts include assessment of waste minimization opportunities and the development of source reduction plans. The projected budget authority goals of the waste minimization program as included in the Environmental Restoration and Waste Management Program are indicated in the following table for LBL's unconstrained funding case.

Waste Minimization Management Program (FY BA \$M) ${ }^{\mathrm{a}}$

\begin{tabular}{lcccccccc}
\hline Category & 1993 & 1994 & 1995 & 1996 & 1997 & 1998 & 1999 & 2000 \\
\hline Operating & 0.2 & 0.2 & 0.4 & 0.4 & 0.4 & 0.4 & 0.5 & 0.5 \\
\hline
\end{tabular}

aEstimated ERWM (EX) Budget Authority for unconstrained funding case.

\section{National Environmental Policy Act Plan}

The Laboratory has undertaken a program to achieve full compliance with the National Environmental Policy Act (NEPA) and the California Environmental Quality Act (CEQA). Consistent with the Office of Energy Research's goals for adherence to the principles of the National Environmental Policy Act, LBL's NEPA Program, in concert with other LBL Environment, Health and Safety programs, strives to: (1) prevent and eliminate damage to the environment from LBL activities; (2) attain beneficial uses of the LBL environment and site without degradation; (3) reduce the risk of undesirable or unintended environmental consequences of $L B L$ activities; and (4) thus, achieve productive harmony between LBL's mission and the environment. The Laboratory has prepared Guidelines for Compliance with the National Environmental Policy Act and the California Environmental Quality Act that describe processes and procedures for LBL scientists and managers to ensure compliance and to prepare documents and recommendations for DOE and for the University of California, Office of the President (UCOP).

LBL's general plan of action for projects includes preparation of checklists specifying NEPA and CEQA documentation recommendations for Field Task Proposals and capital projects one to two years prior to funding, and for Work for Others and Cooperative Research and Development agreements, and research grant proposals at the time of proposal preparation. The checklists are prepared by Division NEPA/CEQA Coordinators, in consultation with principal investigators, and are submitted to DOE in budget documents and to the LBL Office for Planning and Communications for preparation and submittal of formal NEPA and CEQA documentation to DOE and UCOP.

During 1994, the following NEPA Environmental Assessments were under development: the proposed Human Genome Laboratory, Recycling Surplus Copper, and the Building 64 Office and Laboratory Renovation Project. 


\section{Environmental Restoration and Waste Management}

LBL environmental management site projects supported through the DOE Office of Environmental Restoration and Waste Management (EM) are essential to correct and restore environmental conditions at the Laboratory and to improve the management of waste handling operations in support of DOE's national environmental objectives. The corrective actions achieve and maintain required low exposure and risk levels. The environmental restoration program includes the assessment and characterization of contamination and the closure of the existing Hazardous Waste Handling Facility. Increased support for the waste management program is necessary for the proper management of radioactive and hazardous waste. The waste management program supports the construction of a new Hazardous Waste Handling Facility. These two programs meet for compliance with DOE and other federal regulations, as well as requirements established by state and local agencies.

The Laboratory's systematic and prioritized input to the EM Five-Year Plan supports DOE's national environmental restoration and waste management goals. The plan responds to specific environmental conditions at the Laboratory and includes facilities and operating programs for managing those conditions to maintain air quality, surface water quality, and groundwater quality. The plan focuses on three Environmental Management programs for restoration and management activities:

- Environmental Restoration. Assessment, characterization, and remediation of chemical contamination of soils and groundwater, closing of the existing LBL Hazardous Waste Handling Facility, and decommissioning of the Bevalac facility.

- Corrective Activities. The more significant corrective-action projects are associated with an EPA Finding of Violation for noncompliance with Clean Air Act requirements regarding the evaluation of potential radioactive air releases. Subsequently, a Federal Facility Compliance Agreement was established between EPA and DOE. These projects will bring LBL into full compliance by February 1, 1995.

- Waste Management. Waste management program for hazardous and radioactive waste handling, disposal, waste minimization, planning, and the construction of a new Hazardous Waste Handling Facility. Current funding projections as shown in the following table are adequate for meeting regulatory requirements. However, increased funding of waste management operations will be necessary to meet additional program requirements.

EM's Five-Year Plan is vital for compliance with DOE and other federal regulations and for meeting requirements established by state and local agencies. The program has been developed in conjunction with DOE, state, and federal reviews. The resource projections below and in Section VI reflect existing guidance in Activity Data Sheets. These resources do not include additional corrective action planning requirements, maintenance projects, and other upgrades funded through LBL institutional resources. The resources also do not include the cost for decommissioning the Bevalac, following acceptance by EM. 
Environmental Restoration and Waste Management Resource Requirements $(\$ M)^{\mathrm{a}}$

\begin{tabular}{lrrrrrrl}
\hline Category & 1994 & 1995 & 1996 & 1997 & 1998 & 1999 & 2000 \\
\hline Environmental Restoration & & & & & & & \\
$\quad$ Operating & 3.6 & 4.4 & 4.7 & 4.6 & 2.5 & 2.1 & 2.2 \\
$\quad$ Capital Equipment & 0.0 & 0.0 & 0.0 & 0.0 & 0.0 & 0.0 & 0.0 \\
GPP & 0.0 & 0.0 & 0.0 & 0.0 & 0.0 & 0.0 & 0.0 \\
$\quad$ Line Items & 0.0 & 0.0 & 0.0 & 0.0 & 0.0 & 0.0 & 0.0 \\
$\quad$ Total & 3.6 & 4.4 & 4.7 & 4.6 & 2.5 & 2.1 & 2.2 \\
EM Corrective Activity & 0.0 & 0.0 & 0.0 & 0.0 & 0.0 & 0.0 & 0.0 \\
Waste Management & & & & & & & \\
$\quad$ Operating & 6.9 & 8.7 & 9.3 & 6.8 & 6.9 & 7.1 & 7.2 \\
$\quad$ Capital Equipment & 0.8 & 0.6 & 0.2 & 0.2 & 0.2 & 0.2 & 0.2 \\
$\quad$ GPP & 0.0 & 0.0 & 0.0 & 0.0 & 0.0 & 0.0 & 0.0 \\
$\quad$ Line Items & 5.8 & 0.6 & 0.2 & 0.0 & 0.0 & 0.0 & 0.0 \\
$\quad$ Total & 13.5 & 10.0 & 9.7 & 7.0 & 7.1 & 7.3 & 7.4 \\
Total EM Funding & 17.1 & 14.4 & 14.4 & 11.6 & 9.6 & 9.4 & 9.6 \\
\hline
\end{tabular}

actual-year LBL. Budget Authority as provided in the EM Five-Year Plan. The funding level shown for FY 1994 reflects the actual approved budget, for FY 1995 reflects the President's budget request to Congress, and for FY 1996 reflects the target (EM requested) budget level. Proposals for decommissioning of Bevalac accelerators not included (see subsection on Site and Facilities in this section, below, for more details).

\section{Landlord Funded ES\&H}

ES\&H (other than Waste Management and Site Restoration) is currently funded mostly through Laboratory overhead. Proposals for replacing overhead have been submitted through the ES\&H Management Plan. In 1994 , LBL received $\$ 170,000$ in operating funds through the Multiprogram Energy Laboratory Facilities Support (MEL-FS) program, and this funding support is anticipated to increase to $\$ 1$ million in FY 1995. In addition, the MEL-FS program has also provided support of $\$ 100,000$ for Capital Equipment and approximately $\$ 4$ million for line-item projects.

The ES\&H Management Plan makes the distinction between core activities and additional funding necessary to fully implement an ES\&H program that will address needed improvements facing the Laboratory. This year's Activity Data Sheet submissions are summarized in the table "Management Plan Projections" on page 5-18.

Additional funds have been provided by the Laboratory to cover Tiger Team activities. A very small amount of funds was received for Tiger Team activities in FY 1993 and FY 1994. 


\section{$\because$ MANAGEMENT PRACTICES}

A key element of LBL's strategic plan addresses the need for efficient and effective management practices that focus on performance and accountability. LBL is working with DOE and the UCOP to fully implement performance-based contracting and to streamline management systems. LBL fully supports DOE's Strategic Plan, which addresses the numerous separate systems for managing its operations and improving the Department's flexibility. LBL is working with DOE through Process Improvement Teams and through other management and communications systems to achieve DOE goals to become a more streamlined and agile organization. $L B L$ is taking specific steps to address DOE's defined need for change in information systems, procurement and contracting, planning and budgeting, financial management, and site and facilities management.

\section{Quality Assurance and Self-Assessment Programs}

LBL has a strong tradition of research productivity and quality, maintaining a commitment to the success of DOE's mission and its research program. The Laboratory is committed to continuous improvement in program performance and environment and safety management, and to the execution of best business practices. LBL has developed a strategy for programs to improve performance based on nationally recognized criteria and Total Quality Management principles, and is working with DOE on implementation of these programs.

The Laboratory is responsible for maintaining the infrastructure for effective financial and administrative performance. LBL is committed to best business practices and continuous improvements in all areas of administration, including human resource development and training, finance and contractual relations, materials management and procurement, and facilities maintenance and engineering. The University and the Laboratory emphasize the application of performance measures in key functional areas, and are committed to working in partnership with DOE for continuous performance improvement for national research institutions.

The LBL Operating and Assurance Program (OAP) Plan is the institutional document that specifies the Quality Assurance requirements for the Laboratory. LBL's OAP was developed using the guidance provided in the Implementation Guide for QA Programs for Basic and Applied Research (DOE-ER-STD 600092) and is intended to optimize the long tradition of LBL excellence in science by applying required management controls to both performance of research and to research support activities in an integrated and cost-effective way. In both cases, the extent and detail of the management systems are commensurate with the scale, cost, complexity, and hazards of the work being performed. The requirements specified by the OAP are intended to meet the requirements of DOE Order 5700.6C, Quality Assurance. The Plan describes a management system and set of activities designed to:

- Maintain the level of performance necessary to achieve LBL's programmatic and administrative objectives effectively and safely through the application of Quality Assurance principles.

- Implement an LBL management philosophy that supports and encourages continuous improvement in performance and quality at the Laboratory. 
- Provide a management system that permits an integrated approach to compliance with applicable and related regulatory requirements and DOE orders.

LBL has also instituted a program for self-assessment as a systematic way to identify LBL's strengths and weaknesses, and to develop corrective actions if needed. Each LBL Division develops its own program to evaluate itself against performance objectives established by the Laboratory. This continuous process of information gathering enables LBL to assess performance in a systematic and uniform manner, and to target areas that may need improvement.

\section{Administrative Management}

\section{Financial Management}

The LBL Financial Management organization is responsible for the financial functions of the Laboratory. These include planning for, utilizing of, and accounting for the funds provided to LBL to carry out its research and development programs. Under the Chief Financial Officer there are two major areas: Financial Management/Finance and Accounting, and the Financial Management/Budget Office.

Financial Management/Finance and Accounting is responsible for conducting and overseeing financial operations in a manner that is responsive to the Laboratory's research mission, and complies with UC and DOE regulations, Generally Accepted Accounting Principles, and Federal Cost Accounting Standards. The Financial Management/Budget Office is responsible for budget formulation and execution, budget planning and analysis, and indirect budget planning and monitoring. These areas continue to improve and enhance the Laboratory's financial systems and budget formulation and execution processes.

With respect to financial systems in recent years, substantial improvements have been made and are continuing to be made in streamlining . interactive processes from laborious, time-consuming, multistage hard copy inputs to electronic PC accessibility for Laboratory employees. Results are reflected in the reduction of time it takes to complete actions-e.g., the time to complete some 20,000 annual general ledger account changes has been reduced from as long as six weeks in a given transaction to overnight; and conversion in the handling of over 50,000 annual employee time cards to instantaneous, electronic transactions. A new accounts payable system was implemented, and plans are in place to tie the system into a purchasing-receiving system currently under development. The Laboratory's other immediate projects include developing a general ledger system to handle its 550;000 ledger and 5,600 Financial Information Systems transactions and replacing its existing payroll and personnel system with a new UNIX/Oracle-based system.

With respect to the Laboratory's budget formulation and execution processes, the Financial Management organization conducts an annual workshop for research divisions that is supplemented with a budget preparation handbook; validates budget proposals for adequate justification; and coordinates the Laboratory Director's formal review-discussion process. In the indirect area, the Laboratory's cost structure and indirect rates have been revised to comply with the Cost Accounting Standards. 


\section{Materiel and Site Logistics}

The Materiel and Site Logistics Department has four major functions: (1) material operations; (2) procurement; (3) property management; and (4) site access services. The purpose of the Department (formerly named Business and Materiel) is to procure, store, issue, transport, and distribute materials, and to acquire construction and services necessary to support the Laboratory. The Department is also responsible for overall property management of accountable equipment and supplies. In addition, the Department provides bus transportation support to employees and processes in-bound personnel and visitors through a Reception Center. Administration of the Protective Services contract with the University of California Police Department also falls under Materiel and Site Logistics.

Major challenges continue to face the Department in terms of inefficient and outdated processes and systems. These need to be replaced with a modern overall material tracking system, which includes electronic data interchange with vendors who have cost-effective, bar-coding, and just-intime contracting systems. This is particularly important in light of continuing budget limitations.

Much of more recent quality management thinking focuses on the need for quality improvements to be expressed in terms of purpose as well as in terms of activity. While continuous improvement in and of itself often leads to increased productivity and greater customer acceptance, the real purpose of Total Quality is best achieved by focusing on the need for functional areas to become competitive and to then maintain that competitive posture. In terms of customer relations, the definition of quality might be best stated as follows: "Meeting customers' expectations at prices that they can afford and when they need a product or service." Three separate requirements must be met by Materiel and Site Logistics to successfully achieve this. First, it must meet the specifications of the customer in terms of usability and functionality. Second, the service and/or product must be economically produced or provided, and perceived as such by the customer. Finally, the service/product must be supplied on time to be of proper use.

Accordingly, Materiel and Site Logistics must measure success on the basis of whether we can compete with commercial business entities in the surrounding area that provide like products and/or services. Each support function at LBL must be capable of competing with those "off-the-hill" business firms in the three areas discussed above. Comparisons must be made regularly to ensure that it is cost effective to maintain functions inhouse. This is most cornmonly referred to as making "Make or Buy" decisions. The underlying principle is that "functions should be performed by those best equipped and able to perform them." Of course, factors other than cost must be considered in such decisions, including customer convenience, contractual administration costs, and others. All of these are part of the cost-benefit analysis and evaluation conducted at regular intervals.

\section{Internal Auditing}

The LBL Internal Audit Services Department, established during FY 1993, provides independent and objective reviews and analyses to assist management in achieving internal control objectives. Audit resources are allocated on the basis of a formal risk analysis process. Audit efforts are proactive whenever possible and include involvement in the development of new systems. Topics considered for evaluation encompass all facets of 
the Laboratory, including: Financial Management; Procurement Management; Human Resources Management; Construction and Facilities Management; Information Management; Environmental Health and Safety; Data Processing Systems; Property Management; Planning and Budget Management; and Support Services.

LBL is a participant in the DOE Cooperative Audit Strategy, emphasizing improved communication and coordination among the DOE Offices of Inspector General, DOE Field Offices, and internal audit staffs. This quality initiative should result in greater reliance on the work of internal audit staffs, and in reduced DOE audit effort and oversight. During the past year, LBL Internal Audit Services led the development of an Internal Audit Peer Review Program for DOE management and operating contractors. This program provides assurance that professional auditing standards are complied with and acts as a forum for the exchange of Best Practices to assist in meeting the challenges imposed by changes in strategic direction, increased public scrutiny, and limited DOE resources.

\section{Information Resources Management Program}

The LBL Information Resources Management (IRM) program includes both research and operational activities. Research is discussed in the Applied Mathematics and Computer Science program description (pp. 4-24 and 4-25). Operational activities involve the integration of these new technologies into a seamless information technology infrastructure that supports the research and administrative requirements of the Laboratory.

All scientific programs at LBL make essential use of $L B L$ information resources for the collection and analysis of data and the modeling and visualization of physical process; for numerous administrative support functions; and for communication with colleagues-both on an individual basis through e-mail, fax, and telephone services and through hardcopy and electronic (e.g., World Wide Web) publications, conferences, and video conferences.

This section identifies the major objectives of LBL's IRM efforts and the principal strategies for achieving those objectives.

\section{Major Objectives}

The Laboratory has developed IRM objectives that will support the National Information Infrastructure (NII), encourage the development of usable tools for all LBL employees, foster a corporate approach to information management, encourage the transition from a paper-based information society to one based on electronic media, and promote the development and maintenance of a comprehensive records management program. In somewhat more detail, these goals are:

- For corporate data: Define and implement a corporate information management architecture that includes interoperable electronic mail, makes corporate databases fully accessible, implements file portability, and strengthens institutional standards for database systems and utilities. (Infrastructure)

- For Industrial Partnership (AMTEX): Develop a set of operating principles and enterprise interactions in the Integrated Textile Industry that will enable the system to achieve a sustainable market share. (Programmatic) 
- For the management of scientific and technical information: Expand and extend our current lifecycle information management program to accommodate the increasing shift from a paper-based environment to a network-based environment. (Infrastructure)

- For the NII: Conduct an advanced research and development program in support of the nation's High Performance Computing and Communications Initiative and the most effective development and utilization of national information highways. (Programmatic)

- For on-line information: Encourage the development of on-line access to information located both locally and at other sites; assist in the development of DOE policy governing electronic information; create a library "without walls"; promote the introduction of digital technology into the preparation and presentation of technical material; and contribute to the development of the World Wide Web. (Infrastructure)

- For records management: Maintain a secure records management program that identifies and protects the essential scientific and administrative records of the Laboratory and administers appropriate retention schedules for all LBL records. (Infrastructure)

- For the staff: Provide state-of-the-art and transparent computing and communications resources for DOE programs and service to every scientific, engineering, or administrative employee. These services include advanced network communications technology that keeps pace with demand; workstation support services and technical support for telecommuting and telework; transparent access to computing resources; upgraded central computing facilities; and proper control of access to sensitive files. (Infrastructure)

These goals underpin LBL's mission for research and development, design and operation of user facilities, education and training, and technology transfer. Together with the human and facilities resources of the Laboratory, the information resources enable a flexible and responsive operating environment for the implementation of DOE programs. Effective information management is vital to the success of this mission, and will require the allocation of adequate DOE resources for effective implementation.

Information Resource Management Resource Requirements (FY BA, \$M) ${ }^{\mathrm{a}}$

\begin{tabular}{lrrrrrr}
\hline Category & 1995 & 1996 & 1997 & 1998 & 1999 & 2000 \\
\hline Recharge & 18.10 & 19.01 & 19.96 & 20.96 & 22.00 & 23.10 \\
Overhead & 3.10 & 3.30 & 3.40 & 3.60 & 3.80 & 3.96 \\
\hline
\end{tabular}

aFor those activities identified above as "Infrastructure." 


\section{Strategies}

LBL's strategy for IRM includes focused support for improving the nation's computing and communications infrastructure, assistance to DOE in the development of IRM policies and plans, and development of LBL's computing and communications infrastructure to support the LBL DOE multiprogram energy research Laboratory mission. LBL's strategies directly support IRM goals and objectives described above to provide state-of-theart, transparent computing and communications resources; accessible corporate information management systems; effective scientific and technical information services; and a secure records management program.

Improve National Computing Infrastructure. LBL has provided continuing support to the Office of IRM Policy to develop DOE's Information Resources Management strategies. This includes review of existing strategies and policies and the development of new policies to cope with the changing IRM environment. LBL was active on the IRM Planning Process Improvement Team through direct participation in the Team itself, and continues to participate in the pilot program instituted by OER to test various methods for increasing participation by program managers in the planning process. LBL strongly supports DOE's efforts to develop a planning process that is driven by programmatic need rather than by reporting requirements.

LBL is active in several branches of the standards-development process that are of particular interest to DOE. These include participation in technical committees dealing with network issues, the planning and development of the Energy Sciences Network (ESnet), and the Internet Engineering Task Force.

LBL also supports the development of the national communications infrastructure by providing the chairperson of the ESnet Steering Committee, which assists in the prioritization of DOE developments in highspeed data transfer, video conferencing, and other network-based communications media.

Enhance International Collaborations and Information Environment. In support of DOE and Laboratory goals to increase the benefits of international cooperation in basic science, LBL engages in many activities that involve foreign collaborations. Among those that are IRM-intensive are activities involving LBL's expertise in the numerical modeling of geophysical and geochemical processes. For example, there are joint projects on nuclear or chemical waste with Russia, Sweden, Switzerland, and Canada, and formal agreements with both Mexico and Italy on geothermal development. LBL is the site of the International Geothermal Association. LBL is also a contributor to the extensive international electronic information exchange known as the World Wide Web.

Enhance the LBL Work Environment and Corporate Information. A new institutional strategic change to achieve the LBL Vision 2000 has been an increase in activity directed toward the definition and management of corporate and institutional data. LBL's site strategy encourages 
the integration of appropriate information technology into the individual work environments. The process of creating an integrated Laboratory approach to information management has begun. This effort will extend over several years. Activities in FY 1994 have included Oracle DBMS applications in electronic time reporting, chemical inventory, and waste tracking. Oracle-based commercial databases for Procurement and Human Resources were also acquired. Client-server technology was used for a sample analysis application in the Environment, Health and Safety Division. The ability to join tables from independent databases was demonstrated.

Many of LBL's computing and communications facilities and planning requirements are institutional, and we believe that DOE should provide more vigorous support for institutional technology resources at the multiprogram energy laboratories. This strategy strongly supports the goals of DOE's Strategic Plan 2000 because it reinforces the notion of institutionalization of IRM Planning, which is obscured by a reporting process that requires the partitioning of institutional requirements into a welter of small, fictitious, "programmatic" tabulations.

Convert to a Primarily Electronic Information Environment. LBL has made a number of preliminary steps towards creating an information environment that is primarily on-line rather than paper-based. Over the next several years we shall bring these efforts together so that all LBL staff have convenient on-line access to essentially all the information they need, whether it is located at LBL or at other institutions. This effort will include administrative applications (such as electronic time reporting), digital photography, the exchange of scientific and general-interest information through the World Wide Web, access to remotely located computational resources through ESnet, the expansion of on-line library services, and video conferencing, both desktop and studio-based. It is expected that this new on-line environment will materially change the way in which intellectual and administrative work is accomplished in the years ahead.

Provide Quality and Timely Information and Records. In support of LBL Vision 2000 and DOE's renewed emphasis on quality, LBL is placing increased emphasis on a quality of operations consistent with DOE Orders 5480.19 (Conduct of Operations) and 5700.6B (Quality Assurance). These orders require the Laboratory to ensure that suppliers to LBL meet acceptable quality standards. LBL is seeking to implement this for all information resources and related services, including telecommunications and printing services. LBL encounters problems with GPO-approved nationally solicited printers who fail to meet quality standards in the initial press run. This leads to publication delays, often compounded by time-consuming travel to conduct press inspections. LBL will continue to work with DOE to resolve the problem and enable the timely preparation of quality technical documents.

\section{Resources and Initiatives}

LBL IRM initiatives encompass a range of significant new programs that will expand DOE computing and communications resources. The National Information Infrastructure's Networking Initiative is described in Section IV. The following initiatives are directed at improving DOE and Laboratory infrastructure and management and are directed towards meeting the resource and management needs of the DOE and its Office of Energy Research. 


\section{Advanced High-Speed Networking}

To meet projected traffic and demand, LBL anticipates that expanded communications and networking infrastructure will be required during the middle of this decade. The Laboratory's High Speed Networking Initiative seeks to extend high-speed ( $100 \mathrm{Mbs}$ and beyond) network access to the majority of LBL workstations. In addition, network backbones and server systems will need to be upgraded or replaced.

Advanced High-Speed Networking Resource Requirements $(\$ M)^{a}$

\begin{tabular}{lccccccc}
\hline Category & 1995 & 1996 & 1997 & 1998 & 1999 & 2000 & Total \\
\hline Operating & 0.8 & 0.7 & 0.3 & 0.2 & 0.2 & 0.2 & $2.4^{\mathrm{b}}$ \\
Construction & 0.0 & 0.0 & 0.0 & 0.0 & 0.0 & 0.0 & 0.0 \\
\hline
\end{tabular}

aPreliminary estimate of LBL actual year budget authority (B\&R code $\mathrm{KCO}$, tentative).

blncludes $\$ 1.3 \mathrm{M}$ in equipment costs.

\section{Visualization, Video Conferencing, and Technical Information}

LBL is playing a lead role in organizing a testbed that will bring together most of the major workstation vendors, several universities and laboratories, and the telecommunications industry to develop and test the hardware and software technology needed to support workstation-based video teleconferencing in a metropolitan area network. The project will also develop prototype technologies for teleseminars.

LBL is also playing a lead role in the development of packet-based computing and communication environments to support collaborative work. This includes a multimedia environment incorporating audio, video, and a distributed electronic whiteboard operating over the Internet/ESnet, as well as a packet-based video-conferencing multicast environment that will greatly enhance ER-wide video conferencing.

LBL is continuing to introduce new technology into the operations of the Library and the Technical and Electronic Information Department. The Digital Darkroom, which began full operation at the end of FY 1994, has replaced on-site wet-chemistry photography.

LBL regularly participates in video conferences with collaborators, the UCOP, and DOE Headquarters. We have introduced Mosaic and the World Wide Web into the review of DOE directives, and expect to participate in the initial trials of the World Wide Web as a vehicle for distribution of IRM planning information. LBL also expects to provide production access to desktop video conferencing tools, beginning with limited availability in FY 1995, and becoming generally available by FY 1998.

Technical Information Resource Requirements $(\$ M)^{a, b}$

\begin{tabular}{lccccccc}
\hline Category & 1995 & 1996 & 1997 & 1998 & 1999 & 2000 & Total \\
\hline Operating & 0.5 & 0.3 & 0.2 & 0.2 & 0.2 & 0.3 & 1.7 \\
Construction & 0.0 & 0.0 & 0.0 & 0.0 & 0.0 & 0.0 & 0.0 \\
\hline
\end{tabular}

aPreliminary estimate of LBL actual year budget authority ( $B \& R$ code $K C 07$ ).

${ }^{b}$ Does not include infrastructure activities. 


\section{Other Initiatives and Infrastructure Investments}

In FY 1993 LBL began a program to assess the implications of telecommuting for scientific environments such as exist at DOE laboratories. As a separate initiative, and in common with other federal facilities, LBL has begun an examination of options to satisfy the impending narrowed bandwidth requirements for radio systems. Conversion to narrow bandwidth telecommunications using a trunk-based system will require expenditures over the next several years, beginning in FY 1996. This program is to be coordinated among the DOE laboratories in response to the DOE requirements.

In FY 1995, LBL is beginning a formal program of support for development of the information infrastructure. Some of the initial activities are described above. Other possibilities include the development of a cadre of experts skilled in preparation of multimedia presentations and documents, the introduction of virtual reality concepts into the analysis of complex data, and a sophisticated mechanism for the indexing, storage, and retrieval of visual information using visual and textual search techniques.

Other Initiatives and Infrastructure Investments Resource Requirements $(\$ M)^{a, b}$

\begin{tabular}{lccccccc}
\hline Category & 1995 & 1996 & 1997 & 1998 & 1999 & 2000 & Total \\
\hline Operating & 0.3 & 0.5 & 1.0 & 1.5 & 1.5 & 1.5 & 6.3 \\
Construction & 0.0 & 0.4 & 0.0 & 0.0 & 0.0 & 0.0 & 0.4 \\
\hline
\end{tabular}

apreliminary estimate of LBL actual year budget authority ( $B \& R$ code $K C 07$ ).

boes not include infrastructure activities.

\section{Site and Facilities}

Lawrence Berkeley Laboratory is located in the hills above the UC Berkeley campus, on a 54-hectare (134-acre) site overlooking the San Francisco Bay. Founded in 1931 by Ernest O. Lawrence, the Laboratory was moved to its present site in 1940. It is the oldest of the DOE national laboratories, and the only one located adjacent to a major university.

Over the past decades, LBL has continued on a course of diversification that has brought it wide recognition in high-energy and nuclear physics, materials sciences, chemistry, life sciences, and energy conservation research. For LBL to continue its mission as a multidisciplinary national laboratory, it must address some critical issues and opportunities concerning site and facility improvement. The Laboratory strongly supports strategic planning activities that are now taking place as part of a national effort to restore and maintain the nation's scientific infrastructure, and has made these activities an integral part of its own site development process. In addition, LBL is continually vigilant about creating conditions that protect the LBL staff, the public, and the environment.

\section{Site Description and Status}

The first building on the LBL site was constructed in 1940. In FY 1993 the main site included 77 permanent buildings and 121 trailers and temporary structures. Over $61 \%$ of the permanent buildings are over 25 years old. The existing facilities and the currently funded construction at LBL constitute $182,000 \mathrm{gsm}$ (1.95 Mgsf) located on the main site, the UC campus, and leased off-site locations. The space distribution upon completion of current projects in FY 1994 is shown below.LBL space in campus buildings is 
Age distribution of permanent mainsite buildings (excluding proposed FY 1994 construction).

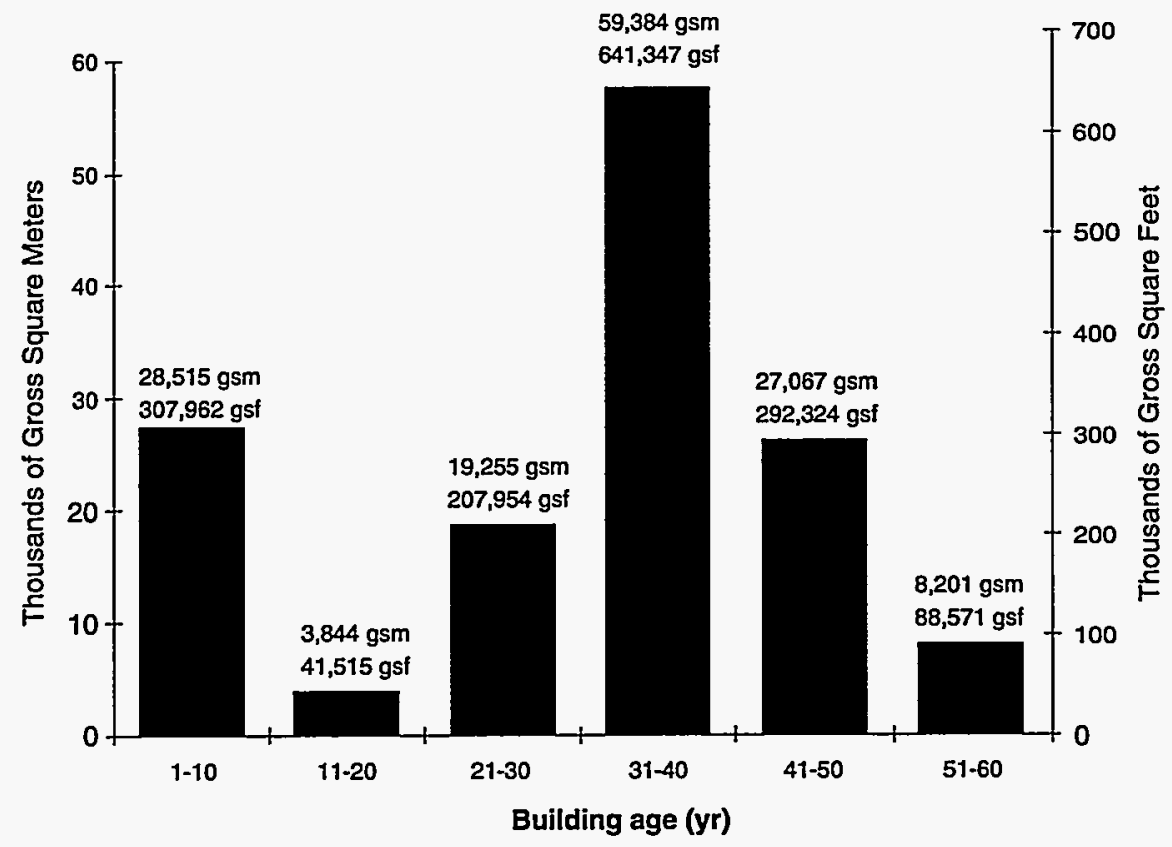

LBL Space Distribution ${ }^{\mathrm{a}}$

\begin{tabular}{lccc}
\hline Location & Area (Ksm) & Area (Mgsf) & \% of Total \\
\hline Main site & 154 & 1.66 & 85 \\
On campus and & & & \\
$\quad$ Richmond Field Station & 10 & 0.10 & 5 \\
Off-site leased & 18 & 0.19 & 10 \\
Total & 182 & 1.95 & 100 \\
\hline
\end{tabular}

ancludes funded projects.

LBL space in campus buildings is available to DOE by a long-term arrangement. The off-site leased buildings provide space for essential research and support functions. Of the $154,000 \mathrm{gsm}$ (1.66 Mgsf) on the main site, about $8,000 \mathrm{gsm}(86,000 \mathrm{gsf})$ are in trailers and other temporary structures. The replacement value of the buildings at the main site, as determined by DOE's Real Property Inventory System for 1994, is more than $\$ 745$ million. Replacement values for utilities and other site improvements continue to be developed. follows:

The inventory of building space, including funded construction, is as

- Rehab Status 1: $51,972 \mathrm{gsm}(558,839$ gsf). Adequate, may require maintenance such as painting, repairs, and minor alterations.

- Rehab Status 2: 82,287 gsm (884,804 gsf). Approaching technological and functional obsolescence but can be economically upgraded to adequate space. 
Condition of Laboratory space (excluding proposed FY 1994 construction).
- Rehab Status 3: 19,877 gsm $(213,734$ gsf). Substandard buildings and temporary trailers that have aged beyond their useful life. Not economically feasible to upgrade to current environmental health and safety standards or functional adequacy.

These categories correspond to former Strategic Facilities Initiative (SFI) building condition categories: Rehab Status 1 (Adequate); Rehab Status 2 (Substandard, Can Be Made Adequate); Rehab Status 3 (Substandard, Cannot Be Made Adequate). The following graph illustrates LBL's inventory of building space. The Condition of Main-Site Space tables on page 5-33 give the specific amounts for each space-type in square meters and square feet.

Facilities Replacement Value ${ }^{\mathrm{a}}$

\begin{tabular}{lcc}
\hline Type & Value $(\$ M)$ & $\%$ of Total \\
\hline Buildings & $272.5^{\mathrm{b}}$ & 64 \\
Utilities & 123.9 & 29 \\
Miscellaneous & 28.8 & 7 \\
Total & 425.2 & 100 \\
\hline
\end{tabular}

aEstimated in 1986.

beplacement value for buildings (1994) is $\$ 745 \mathrm{M}$. Replacement value for utilities and other site improvements are under revision.

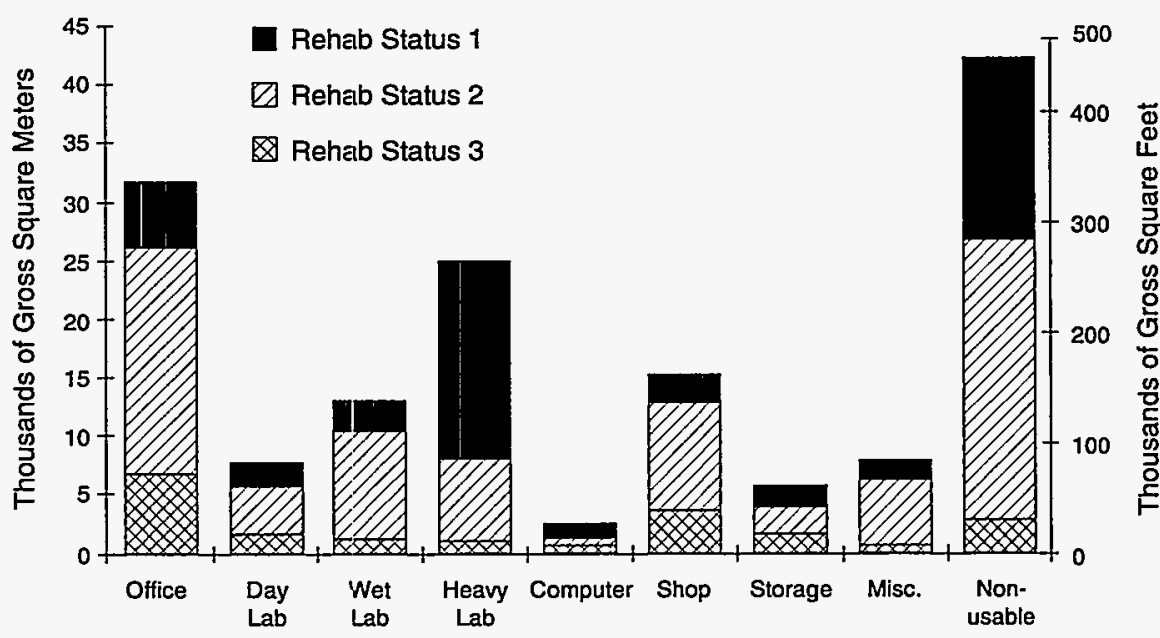


Condition of Main-Site Space by Space Type

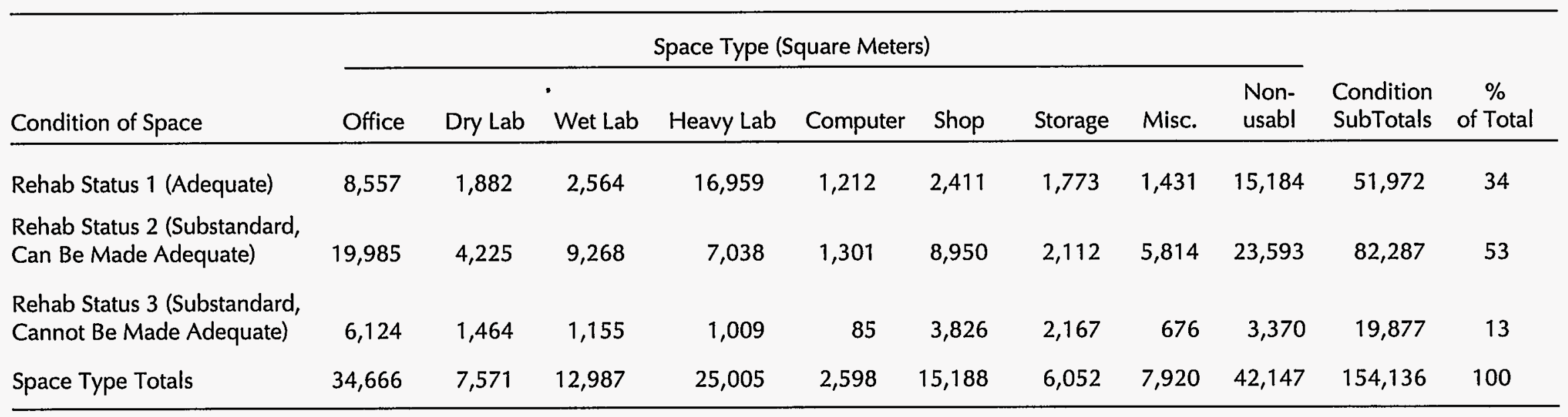

\section{Space Type (Square Feet)}

\begin{tabular}{|c|c|c|c|c|c|c|c|c|c|c|c|}
\hline Condition of Space & Office & Dry Lab & Wet Lab & Heavy Lab & Computer & Shop & Storage & Misc. & $\begin{array}{l}\text { Non- } \\
\text { usable }\end{array}$ & $\begin{array}{l}\text { Condition } \\
\text { SubTotals }\end{array}$ & $\begin{array}{c}\% \\
\text { of Total }\end{array}$ \\
\hline Rehab Status 1 (Adequate) & 92,010 & 20,232 & 27,569 & 182,355 & 13,027 & 25,930 & 19,062 & 15,382 & 163,272 & 558,839 & 34 \\
\hline $\begin{array}{l}\text { Rehab Status } 3 \text { (Substandard, } \\
\text { Cannot be Made Adequate) }\end{array}$ & 65,851 & 15,746 & 12,422 & 10,847 & 918 & 41,140 & 23,305 & 7,270 & 36,235 & 213,734 & 13 \\
\hline
\end{tabular}




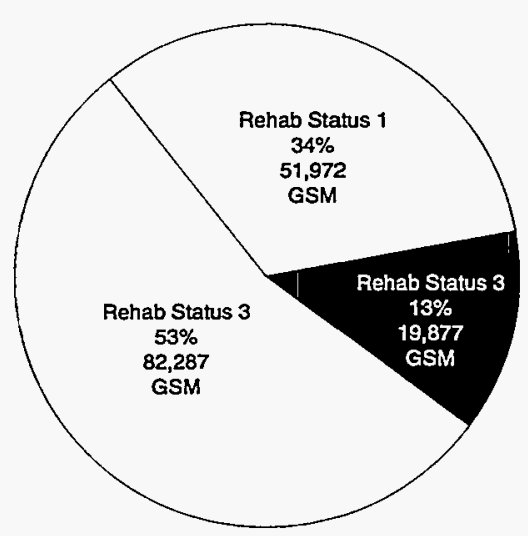

Condition of main-site buildings, including existing construction projects (classified as adequate space).

\section{Facilities Plans and Options}

\section{Site Development Plans}

Each year LBL prepares site-development plans to provide analysis and policy guidance for the effective use and orderly development of land and facilities at LBL. This planning effort is critical to all of the Laboratory's programs. First, it is critical because facilities require rehabilitating to avert safety hazards, shutdowns, and failures. Second, it is critical to optimize use of the Laboratory's limited land and building resources. In addition to the site development plan, department-wide planning efforts such as the Capital Asset Management Process (CAMP) are integrated into the total site-planning effort. The objectives of the site-planning effort are as follows:

- Evaluate future mission projections and anticipate DOE national research facility needs.

- Ensure a safe and healthy workplace in full compliance with building and fire codes.

- Protect the environment and buffer activities to enhance adjacent land uses.

- Protect the national investment in government-owned research and support assets.

- Consolidate research and support services through proper siting of new buildings and maintenance of functional units.

- Work with UC to identify projects with synergistic benefits.

- Make efficient use of unique Laboratory assets and the adaptive reuse of similar facilities with potential to support Laboratory missions.

- Improve access and communications within and to the Laboratory.

- Promote cost reductions and energy conversion through efficiencies in building design and location, operation and maintenance, and parking and transportation.

Resources to improve the Laboratory's facilities are provided through Multiprogram Energy Laboratory Facilities Support (MEL-FS), General Plant Projects (GPP), In-House Energy Management, and General Purpose Equipment (GPE). Adequate funding from these resources is a critical element of LBL's ability to provide DOE with an effective multiprogram Laboratory that can meet environmental and safety standards. A major effort is under way to provide conditions that meet accepted standards for LBL's environment, health, and safety programs, including providing adequate monitoring and sample processing laboratories, emergency command and response facilities, and sufficient space for on-site offices for industrial hygiene, environment, and other essential EH\&S staff discussed above.

LBL's facilities planning is coordinated through specific Laboratory management activities and DOE initiatives. The Site Development Plan (SDP) and the Laboratory Integrated Facilities Plan, which combine the requirements of the Site Development and the Capital Asset Management Process orders, have been updated for FY 1994 and will continue to be 
updated as requested by the Office of Laboratory Management in the Office of Energy Research (ER-80). LBL has established a prioritization framework for its multiprogram capital projects and incorporates a safety and environmental program that supports CAMP. Operating funds have been provided through the MEL-FS General Purpose Facilities KG01 subprogram to support infrastructure, the Condition Assessment Survey (CAS), CAMP and other siteplanning activities. The Laboratory's ten-year In-House Energy Management Plan represents significant opportunities for cost savings. All proposed projects undergo NEPA and CEQA review procedures for full compliance with DOE and UC guidelines. Institutional planning acts to couple site management planning activities to program planning and other strategic management processes. For FY 1994, a complete 20-year infrastructure program has been developed consistent with DOE guidelines and supports the Laboratory's Vision 2000 strategic planning.

The SDP is based on five LBL-site master-plan concepts. These concepts accommodate the facilities improvement needs within existing geophysical, environmental, and operational conditions. They provide a basis for understanding and evaluating the more detailed elements of specific projects, planned locations, and other site-improvement projections. These siteplanning concepts are:

- Consolidation of activities within seven functional planning areas to enhance efficiency and effectiveness and to provide specialized research facilities.

- Redevelopment of obsolete buildings and deteriorated infrastructure, elimination of temporary structures used for permanent functions, and improvement of building arrangements to increase safety and energy efficiency.

- Concentration of development along the east-west circulation and utilities axis to enhance transportation and service systems, e.g., development of off-road parking and improvement of pedestrian pathways.

- Improvement and maintenance of perimeter and internal buffer zones to screen noise-generating activities and minimize potential incompatibility between adjacent operations.

- Providing of off-site facilities for receiving, warehousing, and other support and research activities suitable for decentralization.

Facilities planning includes exploring such options as the potential interim uses of off-site facilities for administrative and other support functions. Over the past three decades administrative requirements have been increasing as safety, accounting, environmental, and other requirements are addressed. LBL is exploring long-term options, such as reconfiguration of some existing space, new additions of priority office and support buildings, and removal of temporary office structures. These temporary office structures proliferated during the past several decades as a result of limited construction for offices and support buildings.

If the full programmatic capability of the site is developed to meet anticipated national needs in energy technology and supporting research, per the Five-Year Plan, the sites and new buildings would result in a net increase of $37,000 \mathrm{gsm}$ ( $0.4 \mathrm{Mgsf}$ ) to the existing main site, for a total of approximately $186,000 \mathrm{gsm}$ ( $2.0 \mathrm{Mgsf}$ ). For comparison, the 1993 total, 
including current construction, consists of $153,000 \mathrm{gsm}(1.65 \mathrm{Mgsf})$ at the main site. The Laboratory's on-site space is at an approximate $70 \%$ net-togross area efficiency. The usable on-site space is approximately 112,000 gsm (1.2M net square feet). The building utilization efficiency is not projected to change significantly, although the efficiency of land use is expected to improve with the replacement of obsolete single- and two-story buildings with three- to five-story structures.

\section{General Purpose Facilities Plans}

Multiprogram Energy Laboratory Facilities Support. The total proposed five-year MEL-FS program represents an investment need of over $\$ 180$ million. This modernization program addresses needs primarily related to the many buildings and utilities that are 30 to 50 years old. Individual projects are evaluated against generic Laboratory site-development priorities such as safety, environmental protection, reliability, maintainability, design standards and obsolescence, and delivery of research and support services. Full modernization of the site would require a long-term 20-year investment program.

Safety and Health Improvements. Health and safety improvements are needed in safety services, health services, building illumination, radiation protection and monitoring, and in other safety systems (see the "Environment, Safety and Health" subsection in this section). Many of these projects were initiated as MEL-FS projects. Examples of important funded projects include Slope and Seismic Stabilization (required in the Bevalac and Shops areas), the Original Labsite Substation Project, the Instrument Support Laboratory Rehabilitation, the Building 90 Seismic Rehabilitation, a Fire and Safety Systems Upgrade Project (Phase I), and the Hazardous Materials Safeguards Program (Phase I).

Road safety improvements are required for safe and efficient operations and movement of staff and materials throughout the site. New facilities for EH\&S operations and laboratories are necessary to correct for overcrowding and substandard space. LBL is re-evaluating ES\&H needs previously defined in safety, support, and environmental facilities to alleviate these conditions. These facilities will allow the Laboratory to meet accepted standards for LBL's environment, health, and safety programs, including providing adequate monitoring and sample processing laboratories, adequate emergency command and response facilities, and sufficient space for on-site offices for industrial hygiene, environmental, and other essential ES\&H staff.

In addition, other safety-related projects are needed to address fire safety and emergency egress. Also, roads need widening, base materials need to be replaced to conform to current standards, acute curves and blind spots need to be eliminated, and road beds and adjacent slopes on steep hillsides need to be stabilized. As an example of long-range safety needs, the plan calls for three phases of road rehabilitation as MEL-FS projects.

Mechanical Utilities/Safety. The Laboratory's mechanical/utility systems are up to 40 years old. Mechanical utilities consist of domestic- and cooling-water, storm-drain and waste, natural-gas, compressed-air, and vacuum systems. Recent MEL-FS funding for Phase I of the Mechanical Utilities Rehabilitation Plan has improved several critical mechanical systems, but nearly $60 \%$ of existing equipment is more than 20 years old and beyond its useful service life. Full implementation of LBL's Six-Phase Plan would minimize the possibility of accidents or program disruption by loss of essential utilities and equipment.

Electrical Utilities/Safety. LBL's power-distribution system consists of 24 
substations and $32 \mathrm{~km}$ ( 20 miles) of $12-\mathrm{kV}$ primary distribution cable. Much of the distribution equipment and cables are obsolete, resulting in reduced reliability and increased maintenance. The electrical rehabilitation projects have been prioritized into several phases based on the expected failure rates of equipment and the importance to site-wide facilities demands. Phase one, Grizzly Peak Substation, has been completed. Phase two, Original Labsite Substation, was funded in 1990, and phase three, East Canyon Electrical Safety Project, was funded in FY 1992. The remaining phases are proposed projects included in the Twenty-Year MEL-FS Plan. The upgraded switching and distribution system will provide the reliability, flexibility, and expandability necessary for efficient Laboratory operation and future growth.

Building Replacement and Modernization. The MEL-FS building replacement and modernization plan responds to the needs for safety, support, and research infrastructures, and for general-purpose engineering facilities. Improvements in substandard space conditions, as part of a long-term modernization program, would enhance the achievement of the Laboratory's mission. Included in the long-range plan is the removal of substandard facilities that cannot be made adequate. Projects include upgrades and additions for facilities, engineering and maintenance services, administrative services, applied sciences, science education, and visitor facilities.

\section{General Plant Projects}

GPP funds are required to address a number of the essential strategic and site-planning objectives of the Department and the Laboratory. The Laboratory applies GPP funds to important environmental, health, safety, and building modernization project needs in an expeditious and cost-efficient manner. Realization of strategic and planning objectives requires capital funds as facility improvements are accomplished. GPP funds are the sole appropriate choice as the cost of these projects is below the MEL-FS threshold. GPP funds support achievement of objectives in many areas, including projects to upgrade high efficiency particulate air (HEPA) filtration systems on glove-box exhaust stacks and to upgrade environmental monitoring equipment capable of documenting the care taken to preserve and protect the environment; to improve slope stability and the use of alternative transportation; and to correct existing noncompliant problems to conform with fire and life safety codes. Additional GPP projects include those to modernize and enhance utility infrastructure; to maintain and advance facility utilization through adaptive reuse of existing assets; and to modernize buildings in order to realize the benefits of automation and technological advances. The Laboratory has a significant backlog of projects, in the amount of approximately $\$ 30$ million. This backlog is not being reduced, as approximately $\$ 3$ million of new project needs are identified annually while as the Laboratory's GPP funding has remained at a similar level for a number of years. In addition, application of overhead further reduces available funds. The Laboratory reviews all candidates on an annual basis, and projects are ranked and reviewed using CAMP and RPM criteria. Management by DOE at the level of the OER facilitates effective resource prioritization and coordination. Increasing GPP funding to $\$ 6$ million annually would ensure achievement of critical strategic and site-planning objectives and reduce the backlog of projects.

\section{General-Purpose Equipment}

Essential support equipment has been funded through DOE. LBL's FiveYear GPE Plan identifies needs based on a range of criteria, including 
environment, safety, and health; legal requirements; failed, worn, inefficient, or obsolete equipment; substandard performance; or increased workload and demand. The current funding level of $\$ 1.7$ million/year is inadequate to meet the Laboratory's needs. As noted in the new DOE/UC contract, "a continuing, active and affirmative program of supplementing and replacing such equipment is essential to scientific progress." Currently, there is a $\$ 19$ million equipment backlog for environmental monitoring, the physical plant, transportation, data processing, and communications. Consolidated GPE management at the level of the OER facilitates the implementation of an integrated and longer-range GPE plan.

\section{Maintenance Plans}

Maintenance plans and budgets are developed annually within an overall five-year planning and safety management strategy. The Laboratory has improved its current maintenance scheduling system and backlog of maintenance projects through implementation of the site-wide Plant Inspection Program and is an active participant in the development of the CAS program. These maintenance plans and budgets include noncapital alterations, general plant projects, and multiprogram general-purpose line items. Requirements are identified by periodic reviews and inspections, and new priorities are developed during the fiscal year.

The operating expenses for maintenance include physical-plant maintenance, mobile-equipment maintenance, and noncapital alternatives related to maintenance. In addition, specialized maintenance related to shop, computer, and telecommunications facilities is also performed.

The current strategy for improving maintenance relies on strengthening the capital outlays, continuing the operating-cost efforts, and implementing the maintenance-planning system. This allows the Laboratory to sustain DOE facilities while planning for maintenance cost economies. These economies can be achieved through the replacement of existing obsolete and high-maintenance-cost facilities with modern facilities and equipment supported by increased MEL-FS, operating, and GPE funds. Operating funds to replace infrastructure equipment are a particularly pressing need because recent revisions to the GPE criteria have eliminated the GPE funding option in this area.

\section{Programmatic Facilities Plans}

The new programmatic research buildings and facilities in the plan serve the national interest in several research areas where LBL has established programs. Several major scientific facilities form the core of LBL's plans to contribute to DOE's research capabilities. The Human Genome Laboratory and ALS Structural Biology Support Facilities are recently funded projects. Proposed are the Chemical Dynamics Research Laboratory, Elise accelerator, ALS Beamline Initiative, and expansion of the National Center for Electron Microscopy-Electron Beam Microcharacterization Facility. Completion of the second floor of the ALS building for users is essential to meet ALS program requirements. The Laboratory is also in the initial stages of planning for an Energy Efficiency and Renewables Facility and a Molecular Design Institute.

\section{Facilities Decommissioning Plan}

The development of new national program directions for nuclear physics resulted in the shutdown of LBL's Bevalac nuclear physics program during FY 1993. We completed a Nuclear Physics stand-down-and-secure of the facility in FY 1993 and FY 1994. LBL is currently working with DOE/ER and DOE/EM 
to obtain a cost-effective and timely recovery of the space previously required for Bevalac operations and experimental programs.

The key element of LBL's plan is a waste minimization initiative for recycling of the Bevalac shielding blocks at the Relativistic Heavy lon Collider (RHIC) at Brookhaven National Laboratory. This proposal leads to significant cost savings to DOE as it averts the disposal as low-level waste of 480,000 cubic feet of shielding block. Thus, use of valuable burial space is avoided, valuable shielding blocks can be reused, and over $\$ 100$ million in burial costs at Hanford are eliminated. A demonstration project to ship 33 roof blocks to Brookhaven was undertaken and successfully completed in FY 1994.

LBL has also developed an alternative plan for Decontamination and Decommissioning of the Bevalac (D\&D; EM-60, 40). This is the broad scope proposal for clearing surplus portions of the site for beneficial reuse by DOE and LBL. The primary scope of work is for the Decontamination and Decommissioning (D\&D) of the Bevatron accelerator and its associated shielding. The scope of this work could be significantly reduced as a consequence of the recycling effort discussed in the previous paragraph.

LBL has developed and submitted several proposals to the Inactive and Surplus Facilities Program (I\&SFP; ER-80). These proposals are for the cleanup and cleanout for eventual reuse of nonactivated Bevalac equipment and peripheral spaces, permitting a gradual return of these spaces to productive Laboratory and programmatic use. Pending funding of the facility cleanup proposals, the Bevalac is in a surveillance-and-maintenance-only mode, funded by the Nuclear Physics Program in OER.

Aside from the Bevalac, the Laboratory conducts periodic reviews of facilities that may become inactive. Other facilities to be decommissioned include gamma irradiators.

Facilities Decommissioning Plan (\$M)

\begin{tabular}{lcccccc}
\hline Category & FY 1995 & FY 1996 & FY 1997 & FY 1998 & FY 1999 & FY 2000 \\
\hline Bevalac: & & & & & & \\
$\quad$ Stand-down and Secure (OER/DNP) & 0.00 & 0.00 & 0.00 & 0.00 & 0.00 & 0.00 \\
$\quad$ Surveillance and Maintenance (OER) & 0.13 & 0.14 & 0.15 & 0.15 & 0.16 & 0.16 \\
$\quad \begin{array}{l}\text { Transition (EM-60) } \\
\text { Decontamination \& }\end{array}$ & 0.00 & 0.00 & 1.15 & 3.00 & 3.00 & 3.00 \\
$\quad \begin{array}{l}\text { Decommissioning (EM-40) } \\
\quad \text { (\$15M in FY 2001) }\end{array}$ & - & - & - & - & - & - \\
$\quad$ Return on Investment (EM-352) & 0.50 & 4.00 & 4.00 & 2.30 & - & - \\
$\quad \begin{array}{l}\text { D\& Characterization Technology } \\
\quad \text { Center (EM-50) }\end{array}$ & 0.52 & 0.54 & TBD & - & - & - \\
$\quad$ Inactive \& Surplus Facilities & 0.20 & 0.16 & 0.16 & 0.16 & 0.16 & 0.16 \\
$\quad$ Program (ER-80) & 0.30 & - & - & - & - & - \\
$\begin{array}{l}\text { Gamma Irradiators (OER) } \\
\text { D\&D Base Program (OER) }\end{array}$ & 0.40 & 0.60 & 0.60 & - & - & - \\
$\quad$ Total & 2.05 & 5.44 & 6.06 & 5.61 & 3.32 & 3.32 \\
\hline
\end{tabular}

aTotal estimate to complete the D\&D of the Bevalac by FY 2004 per DOE guidance is $\$ 140 \mathrm{M}$ (representing a $\$ 35 \mathrm{M}$ increase over proposed FY 1998 start for D\&D). Also, no allowance is included for possible savings from other initiatives (e.g., shielding block recycling as discussed above). 


\section{Facilities Resources Requirements}

A five-year construction plan for programmatic and general purpose facilities is provided in the Lawrence Berkeley Laboratory Construction Plan table for FY 1996 to FY 2000 (facing page). MEL-FS proposed projects are listed in a prioritized order of sequence in accordance with CAMP criteria. All budget information as indicated is actual-year authority. 


\section{LAWRENCE BERKELEY LABORATORY CONSTRUCTION PLAN FY 1995 - FY 2000}

Plan for Programmatic and General Purpose Facilities, Including Funded, Budgeted and Proposed Construction (FY BA, \$M)

\begin{tabular}{|c|c|c|c|c|c|c|c|c|c|c|c|}
\hline Project & Scope & TEC & Prior" & 1995 & 1996 & 1997 & 1998 & 1999 & 2000 & 2001 & 2002 \\
\hline \multicolumn{12}{|l|}{ FUNDED PROGRAM RELATED PROJECTS: } \\
\hline 94 Human Genome Laboratory (KP) & $3.809 \mathrm{gsm}(41,000 \mathrm{gsf})$ & 24.700 & 2.134 & 15.800 & 5.766 & 1.000 & & & & & \\
\hline 94 ALS Structural Biology Support Facilities & $1,031 \mathrm{gsm}(11,100 \mathrm{gst})$ & 7.900 & 0.582 & 4.700 & 2.618 & & & & & & \\
\hline 96 Elise (AT) & $595 \mathrm{gsm}(6,400 \mathrm{gsi})$ & 20.200 & & & 5.000 & 5.350 & 5.650 & 4.200 & & & \\
\hline SUBTOTAL-FUNDED PROGRAM RELATED & & 52.800 & 2.716 & 20.500 & 13.384 & 6.350 & 5.650 & 4.200 & & & \\
\hline \multicolumn{12}{|l|}{ FUNDED MEL-FS PROJECTS (KG): } \\
\hline 92 East Canyon Electrical Safety Project & & 3.900 & 2.900 & 1.000 & & & & & & & \\
\hline 93 Fire and Safoty Systems Upgrade Proj., Ph I & & 4.600 & 1.470 & 2.000 & 1.130 & & & & & & \\
\hline 93 Hazardous Materials Safeguards, $\mathrm{Ph} . \mathrm{I}$ & & 4.720 & 1.470 & 1.962 & 1.288 & & & & & & \\
\hline 96 Sanitary Sewer Restoration, Phase I & $1,036 \mathrm{~m}(3,400 \mathrm{ft})$ & 2.400 & & & 0.400 & 1.600 & 0.400 & & & & \\
\hline SUBTOTAL_FUNDED MEL-FS PROJECTS & & 15.620 & 5.840 & 4.962 & 2.818 & 1.600 & 0.400 & 0.000 & 0.000 & 0.000 & 0.000 \\
\hline \multicolumn{12}{|l|}{ FUNDED ERWM PROJECTS: NONE } \\
\hline 88 Hazardous Waste Handling Facility & 1,198 gsm (12,900 gsf) & 12.625 & 11.829 & 0.625 & 0.171 & & & & & & \\
\hline SUBTOTAL_FUNDED ERWM PROJECTS & & 12.625 & 11.829 & 0.625 & 0.171 & 0.000 & 0.000 & 0.000 & & & \\
\hline TOTAL FUNDED & & 81.045 & 20.385 & 26.087 & 16.373 & 7.950 & 6.050 & 4.200 & & & \\
\hline \multicolumn{12}{|l|}{ BUDGETED MEL-FS PROJECTS (KG): NONE } \\
\hline TOTAL FUNDED and BUDGETED & & 81.045 & 20.385 & 26.087 & 16.373 & 7.950 & 6.050 & 4.200 & & & \\
\hline \multicolumn{12}{|l|}{$\begin{array}{l}\text { PROPOSED PROGRAM RELATED } \\
\text { PROJECTS: }\end{array}$} \\
\hline $\begin{array}{l}\text { PROJECTS: } \\
\text { ALS Beamlines Initiative (KC) }\end{array}$ & 1,877 gsm (20,200 gsi) & 54.7 & & & & 12.1 & 24.9 & 17.8 & & & \\
\hline Chemical Dynamics Research Laboratory (KC) & $3,066 \mathrm{gsm}(33,000 \mathrm{gsf})$ & 64.3 & & & & 8.1 & 19.2 & 18.4 & 12.2 & 6.4 & \\
\hline NCEM Electron Beam Microchar Facility (KC) & $900 \mathrm{gsm}(10,000 \mathrm{gsf})$ & 16.5 & & & & 1.0 & 8.1 & 7.0 & 0.4 & & \\
\hline TOTAL-PROPOSED PROGRAM RELATED & & 135.5 & & & & 21.2 & 52.2 & 43.1 & 12.7 & 6.4 & \\
\hline \multicolumn{12}{|l|}{ PROPOSED MEL-FS PROJECTS: } \\
\hline 96 Childcare Facility & $595 \mathrm{gsm}(6,400 \mathrm{gsf})$ & 3.5 & & & 0.4 & 3.1 & & & & & \\
\hline $\begin{array}{l}97 \text { Elec Sys Rehab, Ph IV-BBC Swch Sta } \\
\text { Replc }\end{array}$ & & 8.8 & & & & 0.5 & 23 & 5.0 & 1.0 & & \\
\hline Roof Replacements, Ph II & & 7.5 & & & & 0.5 & 3.1 & 3.3 & 0.6 & & \\
\hline 98 Seismic Safety Improvement Project, Ph I & & 4.0 & & & & & 0.6 & 20 & 1.4 & & \\
\hline Mechanical Equipment Replacement, Ph I & & 4.9 & & & & & 0.5 & 42 & 0.2 & & \\
\hline Environmental and Support Bldg** & & 10.0 & & & & & 0.9 & 4.0 & 4.5 & 0.6 & \\
\hline 99 Building Rehabilitiation, Phase I & & 5.5 & & & & & & 0.5 & 22 & 2.0 & 0.8 \\
\hline Fire \& Salety Systems Upgrd Project. Ph II & & 4.9 & & & & & & 0.7 & 2.5 & 1.7 & \\
\hline Admin Services Addn-BIdg 50E/F 2nd FI & $1,709 \mathrm{gsm}(18,400 \mathrm{gsf})$ & 82 & & & & & & 1.0 & 4.5 & 2.7 & \\
\hline oo Mochanical Utilities Upgrade & & 7.4 & & & & & & & 1.1 & 4.0 & 2.3 \\
\hline Roadway Safety \& Stabilization, Phase 1 & & 6.8 & & & & & & & 0.8 & 3.0 & 3.0 \\
\hline Building Rehabilitiation, Phase II & & 3.5 & & & & & & & 0.5 & 2.0 & 1.0 \\
\hline 01 Roof Replacements, Phase III & & 5.9 & & & & & & & & 0.6 & 2.8 \\
\hline Fire \& Safety Systems Upgrd Project, Ph III & & 5.8 & & & & & & & & 0.8 & 3.0 \\
\hline Facilities Building & 2,657 gsm $(28,600 \mathrm{gsf})$ & 16.6 & & & & & & & & 2.5 & 6.0 \\
\hline SUBTOTAL-PROPOSED MEL-FS PROJECTS & & 103.3 & 0.0 & 0.0 & 0.4 & 4.1 & $7 A$ & 20.7 & 19.3 & 19.9 & 18.9 \\
\hline $\begin{array}{l}\text { TOTAL FUND. BUDGT \& PROP MEL-FS } \\
\text { PROJECTS }\end{array}$ & & 118.9 & 5.8 & 5.0 & 32 & 5.7 & 7.8 & 20.7 & 19.3 & 19.9 & 18.9 \\
\hline
\end{tabular}

-Prior costs from previous years.

"This project incoporates the highest priority needs from the larger and previously submitted Safety and Support Facility, and Environmental Monitoring and Industrial

Hygiene Building candidates. The prioritization and rescoping are currently underway.

Notes: August 1994 escalation rates 1 FY96, 3.8\%; FY97, 3.9\%; FY98, 4.1\%; FY99, 3.9\%; FYo0 and beyond 3.7\%. Overhead $8.61 \%$ on TEC and is subject to change. Long-term projects also under consideration are Isospin Laboratory, Molecular Design Institute, and Energy Efficiency for the Climate Change Action Plan Initiative. 


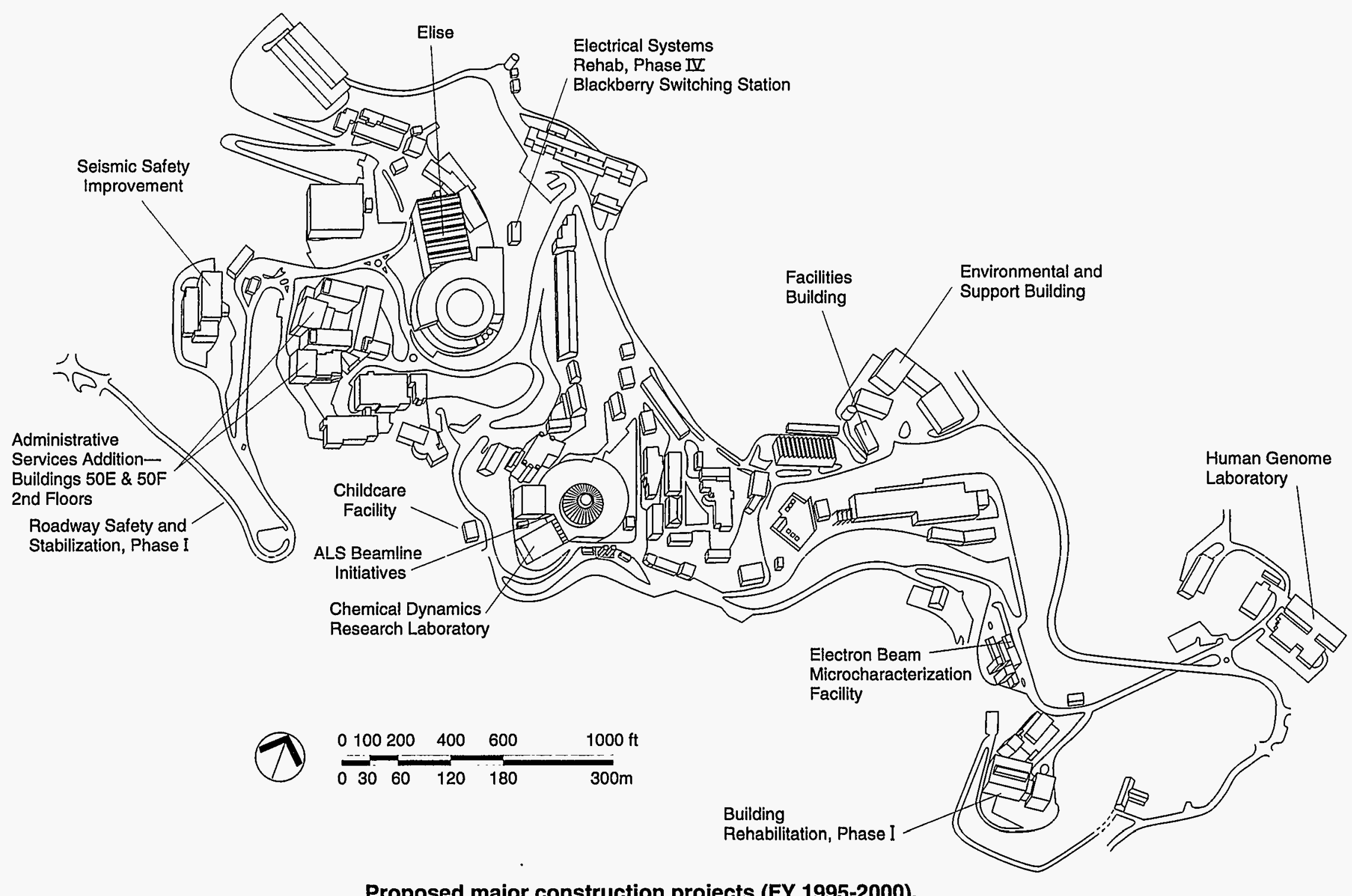

Proposed major construction projects (FY 1995-2000). 


\section{COMMUNICATIONS AND TRUST}

A key element of LBL's strategic plan (summarized in Section III) is a broad initiative to strengthen communications at all levels and to build trust with LBL's customers and stakeholders - focusing initially on several essential areas: enhanced internal communications, improved communications with DOE and federal and industrial partners, and a strengthened identity for the community and region. LBL has prepared an initial communications report defining communications issues and potential strategies and has established a Communications Task Force and Advisory Council to address communications needs. These efforts support DOE's strategic communications planning to transform the Department into a service-oriented, customer-driven leader in science, technology, and environmental management.

\section{Strengthening Communication}

Strengthening communications at all levels, internal and external, to build trust with LBL's customers, stakeholders, and employees is among the key elements of the LBL Strategic Plan. This emphasis parallels the Department of Energy's goal to change its culture to one of openness, communication, and trust in a service-oriented, customer-driven environment.

LBL's history has been one of excellent science and outstanding research achievement, but its public persona has been less clearly recognized. As the public and government demand more accountability for scientific investments and increase their scrutiny in areas of environmental responsibility and safety, improved relationships with local, regional, and national constituencies become necessary instruments of survival. Institutions such as LBL must deal honestly and fairly with the public they serve, to increase knowledge about their mission and activities, and to reduce unwarranted fears. Habits that encourage communication and trust must become part of LBL's culture.

To do this, LBL commissioned an initial report in Fall of 1993 defining communications issues and potential strategies for improving current programs and developing new ones. In 1994, two committees were established to address communications needs-the Communications Advisory Council, a management-level group which serves as counsel to the Director; and the Laboratory Communications Task Force, a working group charged with developing a Laboratory-wide LBL Communications Plan.

\section{Identity and Image}

The LBL strategic plan clearly elucidates a mission and priorities for the Laboratory and thus provides the foundation for development of unifying and consistent written and graphic symbols of LBL's identity. An image program which clearly defines and identifies the elements that make LBL unique will assist in its overall recognition among its many constituencies. One manifestation of the identity program will be a more welcoming and negotiable campus. Consistent graphics and publications guidelines will also lend a sense of unity and familiarity to LBL products and vehicles. Consistent with the mandate of the strategic plan, LBL's identity will evolve from simply one of great science to also one of value and relevance in the pursuit of national problems. 


\section{On-site Communications}

A heightened image and visibility for LBL externally need to begin with a positive and informed community on-site. This includes vertical as well as horizontal communications. LBL will continue to enhance two-way interactions between management and the workforce through training programs for Laboratory leadership, increased opportunities for employee development and feedback, and improved communication mechanisms and programs. To strengthen the sense of community, programs are being designed to encourage participation and involvement among all segments of the Laboratory-educational, social, and recreational-throughout the year.

Integration of electronic communications systems and networks will be essential for effective linkage of LBL personnel and programs, and the development of new and innovative technologies to share information will be among the Laboratory's top planning priorities. A well-informed Laboratory citizenry leads to a more rewarding and safe workplace that nurtures creativity, rewards achievement, and is results-oriented and enjoyable.

\section{Public Information}

How LBL is perceived by its most important external constituenciesBay Area communities, Congress and the Department of Energy, the general and scientific news media, the University of California, and prospective business partners-will be essential to the fulfillment of its mission. An aggressive, market-based, customer-oriented program of publicity and promotion is being developed to heighten LBL's profile and broaden understanding of and appreciation for its work.

Primary audiences include the Bay Area communities, in particular the neighborhoods of Berkeley and Oakland; the undefined "public" reached via the general news media; elected officials and regulatory agencies; the Department of Energy; the University of California; prospective business clients in technology and industry; and members of various scientific communities, reached through professional affiliations or the science press. Prospective employees, especially from minority and underrepresented communities, are particularly important targets to reach as LBL strives to achieve its diversity goals. LBL's continuing relationships with educators in the promotion of science in the schools will be highlighted, as will the many other special partnerships upon which the Laboratory's future successes depend.

In addition to the messages of relevance that will spotlight applied science in the core competencies and their applications to real-world needs, LBL will convey the excitement and value of fundamental research in the problem-solving continuum. Basic science has been the foundation of the Laboratory's record of achievement and reputation, and it continues to reflect the creativity and the pioneering spirit of discovery that underlies the continuing quest to solve the riddles of the universe. The nation's taxpayers, as shareholders in these endeavors, must be kept informed of the benefits of their investments in scientific advancement. LBL's communications goalthrough the proactive utilization of print, electronic, and visual media-is to make the American people aware of this world-class treasure in order to encourage their continuing support. 


\section{Community Relations and Outreach}

Being a "good neighbor" and a valued member of the surrounding community has never been more important to LBL, and thus its communications and interactions with local government, agencies, citizens' groups, schools, the news media, and other stakeholders will be enhanced. This includes continuing efforts to cooperate in the planning of all projects that might involve environmental and cultural impacts on the city and county within which LBL exists.

A comprehensive and effective community relations program requires regular interactions between the citizens of $L B L$ and the external environment. These connections, involving administrators, scientists, and staff members at the Laboratory, will be encouraged through the Speakers' Bureau, the participation of employees in community-based projects and service groups, and the development of a more welcoming Laboratory environment. Visitor programs, special events, and targeted communications vehicles will be developed and promoted to project an inviting, cooperative, user-friendly image of openness and honesty. Goals of the Community Relations Program are identified as follows:

- Integrate community relations activities within the scientific divisions with the overall LBL community relations effort, so that all issues of public concern may be addressed in a coordinated program.

- Continue a two-way communication with the public.

In particular, the local community is active in many environmental issues. Opportunities for public involvement are built into regulations, but LBL will expand these opportunities by encouraging the public to participate in discussions at local community meetings about pertinent issues. Another, more focused goal is:

- Provide the community with accurate and timely information to increase the level of understanding of LBL programs.

The local community, including elected officials, staff, LBL employees, and site neighbors, is very interested in receiving more information about LBL. Several vehicles to orient the public about LBL programs are: site tours and open houses, information repositories, mailing lists, fact sheets and summaries of technical documents, a speakers bureau, and meetings and presentations. The purpose of these is to:

- Respond to the different information requirements of specific groups, including elected officials, city staff, site neighbors, and employees. Activities include: briefings for elected officials, attendance at local community meetings, and an electronic mail notification system for neighboring facilities, including a fax notification system.

- Respond to the changing needs of the community.

LBL's efforts to assist in the delivery of science education in the schools through regional partnerships, participation in special in-school programs, and development of student exchange agreements with other institutions will be maintained and enhanced. Its commitment to the encouragement of science as a career for women and minorities will continue to be a priority in its fulfillment of educational outreach goals. And the communication of these efforts to the greater public audience is essential to LBL's recognition as a valued national and regional citizen. 
LBL values its relations with local communities and is committed to an expanding outreach effort. The benefits of a good community relations program can be seen in the burgeoning partnerships LBL is now forming with the surrounding cities of Berkeley and Oakland. For example, LBL has become an active participant in the city of Berkeley's Environmental Affairs Commission Meetings and its Earth Day celebration. The Laboratory is also a prominent member of a county-wide coalition to formulate a reconversion plan for the Alameda Naval Air Station, which has been scheduled for closure. LBL will bring its scientific and technological expertise to bear on the effort to restore the environment around the base and create new jobs in place of those that will be lost.

Operating under the philosophy that every LBL employee is an ambassador for the Laboratory, the community relations program is directed internally as well. Noon-time lectures and films, nature walks around the Laboratory's scenic premises, ecology fairs, and other activities are held throughout the year, and all employees are encouraged to attend. 


\section{RESOURCE PROJECTIONS}

Resource projections for the Institutional Plan provide a description of the budget authority to implement the research programs. The resource tables also indicate actual FY 1993 BA and estimated FY 1994 BA for comparison. These tables include:

- Resource Summaries (page 6-2);

- Secretarial Level Resources (pages 6-3 and 6-4);

- Program Office Resources (pages 6-5 through 6-8);

- Work for Others Resources (page 6-9); and

- Subcontracting and Procurement (page 6-10).

The FY 1995 estimate is based on FY 1995 DOE budget guidance and assessments by LBL Divisions. For fiscal years 1996 and beyond, operating cost projections are in FY 1996 dollars and construction costs are in actualyear dollars (as indicated in the DOE guidance). For FY 1996 to FY 2000, the growth assumptions in program areas as tabulated range from $0 \%$ to $1.5 \%$ per year. These growth assumptions are based on the general direction indicated by DOE program personnel. Specific trend levels were established within each program activity.

The resource projections that follow include all funded and budgeted construction projects, the projected MEL-FS program, the approved Environmental Restoration and Waste Management program funding and construction, and operational costs for the Advanced Light Source. The new initiative costs are indicated in Section IV. Construction project costs are provided in Section V. The distribution of direct and indirect full-time equivalents is integrated with ES\&H staffing planning, but is insufficient for full support of the 5-year ES\&H Plan and Corrective Action Plan (see Section V), both of which require additional program support for full implementation. 
Funding Summary (Fiscal Year Budget Authority, \$M)

\begin{tabular}{lrrrrrrrr}
\hline Category & 1993 & 1994 & 1995 & 1996 & 1997 & 1998 & 1999 & 2000 \\
\hline DOE Operating & 173.8 & 184.3 & 173.2 & 186.1 & 181.4 & 181.1 & 182.4 & 184.4 \\
WFO Operating & 40.6 & 43.0 & 44.4 & 44.8 & 54.7 & 58.1 & 58.5 & 59.7 \\
$\quad$ Total Operating & $\mathbf{2 1 4 . 4}$ & $\mathbf{2 2 7 . 3}$ & $\mathbf{2 1 7 . 6}$ & $\mathbf{2 2 7 . 3}$ & $\mathbf{2 3 6 . 1}$ & $\mathbf{2 3 9 . 2}$ & $\mathbf{2 4 0 . 9}$ & $\mathbf{2 4 4 . 1}$ \\
Capital Equipment & 29.9 & 34.5 & 34.0 & 31.4 & 24.4 & 23.0 & 23.1 & 23.0 \\
Program Construction & 3.9 & 8.6 & 25.2 & 12.7 & 5.5 & 5.5 & 4.5 & 4.5 \\
MEL/FS-Revitalization & 4.6 & 5.0 & 5.8 & 5.7 & 10.0 & 10.0 & 10.0 & 10.0 \\
Environmental Restoration & 0.5 & 5.7 & 0.6 & 0.2 & 0.0 & 0.0 & 0.0 & 0.0 \\
General Plant Projects & 3.4 & 3.4 & 4.0 & 4.5 & 4.5 & 4.5 & 4.5 & 4.5 \\
General Purpose Equipment & 1.9 & 1.9 & 3.0 & 3.4 & 4.5 & 4.5 & 4.5 & 4.5 \\
$\quad$ Total Lab Funding & $\mathbf{2 5 8 . 6}$ & $\mathbf{2 8 6 . 4}$ & $\mathbf{2 9 2 . 2}$ & $\mathbf{2 8 5 . 2}$ & $\mathbf{2 8 5 . 0}$ & $\mathbf{2 8 6 . 7}$ & $\mathbf{2 8 7 . 5}$ & $\mathbf{2 9 0 . 6}$ \\
\hline
\end{tabular}

Personnel Summary (Fiscal Year FTE)

\begin{tabular}{lrrrrrrrr}
\hline Category & 1993 & 1994 & 1995 & 1996 & 1997 & 1998 & 1999 & 2000 \\
\hline DOE Effort & 1590 & 1527 & 1505 & 1520 & 1532 & 1535 & 1545 & 1559 \\
WFO & 338 & $\mathbf{3 2 8}$ & 325 & 339 & 349 & 354 & 354 & 354 \\
$\quad$ Total Direct & $\mathbf{1 9 2 8}$ & $\mathbf{1 8 5 5}$ & $\mathbf{1 8 3 0}$ & $\mathbf{1 8 5 8}$ & $\mathbf{1 8 8 1}$ & $\mathbf{1 8 8 8}$ & $\mathbf{1 8 9 9}$ & $\mathbf{1 9 1 3}$ \\
$\quad$ Total Indirect & $\mathbf{7 8 7}$ & $\mathbf{7 7 6}$ & $\mathbf{7 6 1}$ & $\mathbf{7 6 4}$ & $\mathbf{7 7 0}$ & $\mathbf{7 7 0}$ & $\mathbf{7 7 0}$ & $\mathbf{7 7 1}$ \\
Total Lab Personnel & $\mathbf{2 7 1 5}$ & $\mathbf{2 6 3 1}$ & $\mathbf{2 5 9 1}$ & $\mathbf{2 6 2 2}$ & $\mathbf{2 6 5 1}$ & $\mathbf{2 6 5 8}$ & $\mathbf{2 6 6 9}$ & $\mathbf{2 6 8 4}$ \\
\hline
\end{tabular}


Secretarial Office Funding Summary (Fiscal Year Budget Authority, \$M)

\begin{tabular}{|c|c|c|c|c|c|c|c|c|}
\hline Office/Program & 1993 & 1994 & 1995 & 1996 & 1997 & 1998 & 1999 & 2000 \\
\hline \multicolumn{9}{|l|}{ Office of Energy Research } \\
\hline Operating & 131.5 & 132.5 & 127.5 & 134.1 & 135.8 & 137.4 & 139.0 & 140.6 \\
\hline Capital Equipment & 20.1 & 22.6 & 16.9 & 17.4 & 18.2 & 18.2 & 18.2 & 18.2 \\
\hline Construction & 10.5 & 15.9 & 33.0 & 21.9 & 19.0 & 19.0 & 18.0 & 18.0 \\
\hline Total & 162.1 & 171.0 & 177.4 & 173.4 & 173.0 & 174.6 & 175.2 & 176.8 \\
\hline \multicolumn{9}{|c|}{ Energy Efficiency and Renewable Energy } \\
\hline Operating & 17.6 & 24.6 & 20.4 & 21.0 & 21.0 & 21.0 & 21.0 & 21.0 \\
\hline Capital Equipment & 0.7 & 1.3 & 0.7 & 0.7 & 0.7 & 0.7 & 0.7 & 0.7 \\
\hline Total & 18.3 & 25.9 & 21.1 & 21.7 & 21.7 & 21.7 & 21.7 & 21.7 \\
\hline \multicolumn{9}{|l|}{ Fossil Energy } \\
\hline Operating & 2.2 & 3.3 & 2.0 & 2.1 & 2.1 & 2.1 & 2.1 & 2.1 \\
\hline Capital Equipment & 0.0 & 0.0 & 0.1 & 0.0 & 0.1 & 0.0 & 0.1 & 0.0 \\
\hline Total & 2.2 & 3.3 & 2.1 & 2.1 & 2.2 & 2.1 & 2.2 & 2.1 \\
\hline \multicolumn{9}{|l|}{ Civilian Waste Management } \\
\hline Operating & 3.3 & 3.8 & 3.5 & 3.6 & 3.6 & 3.6 & 3.6 & 3.6 \\
\hline Capital Equipment & 0.1 & 0.2 & 0.1 & 0.1 & 0.1 & 0.1 & 0.1 & 0.1 \\
\hline Total & 3.4 & 4.0 & 3.6 & 3.7 & 3.7 & 3.7 & 3.7 & 3.7 \\
\hline \multicolumn{9}{|c|}{ Environmental Restoration and Waste Management } \\
\hline Operating & 10.1 & 12.5 & 15.3 & 17.2 & 14.4 & 12.5 & 12.2 & 12.5 \\
\hline Capital Equipment & 0.2 & 0.9 & 0.6 & 0.2 & 0.2 & 0.2 & 0.2 & 0.2 \\
\hline Construction & 0.5 & 5.7 & 0.6 & 0.2 & 0.0 & 0.0 & 0.0 & 0.0 \\
\hline Total & 10.8 & 19.1 & 16.5 & 17.6 & 14.6 & 12.7 & 12.4 & 12.7 \\
\hline \multicolumn{9}{|c|}{ Environment, Health and Safety } \\
\hline Operating & 2.6 & 1.7 & 1.0 & 1.0 & 1.0 & 1.1 & 1.1 & 1.1 \\
\hline Total & 2.6 & 1.7 & 1.0 & 1.0 & 1.0 & 1.1 & 1.1 & 1.1 \\
\hline \multicolumn{9}{|c|}{ Administration and Human Resource Management } \\
\hline Operating & 0.2 & 0.7 & 0.3 & 0.3 & 0.3 & 0.3 & 0.3 & 0.3 \\
\hline Construction & 1.4 & 1.1 & 2.0 & 1.0 & 1.0 & 1.0 & 1.0 & 1.0 \\
\hline Total & 1.6 & 1.8 & 2.3 & 1.3 & 1.3 & 1.3 & 1.3 & 1.3 \\
\hline \multicolumn{9}{|c|}{ Domestic \& International Energy Policy } \\
\hline Operating & 0.8 & 0.5 & 0.2 & 0.2 & 0.2 & 0.2 & 0.2 & 0.2 \\
\hline Total & 0.8 & 0.5 & 0.2 & 0.2 & 0.2 & 0.2 & 0.2 & 0.2 \\
\hline \multicolumn{9}{|c|}{ Work for Other DOE Contractors } \\
\hline Operating & 5.5 & 4.7 & 3.0 & 3.0 & 3.0 & 3.0 & 3.0 & 3.0 \\
\hline Capital Equipment & 6.0 & 6.3 & 5.0 & 3.1 & 3.1 & 3.1 & 3.1 & 3.1 \\
\hline Total & 11.5 & 11.0 & 8.0 & 6.1 & 6.1 & 6.1 & 6.1 & 6.1 \\
\hline \multicolumn{9}{|l|}{ Total DOE } \\
\hline Operating & 173.8 & 184.3 & 173.2 & 182.5 & 181.4 & 181.1 & 182.4 & 184.4 \\
\hline Capital Equip. (inc.GPE) & 27.1 & 31.3 & 23.4 & 21.5 & 22.4 & 22.3 & 22.4 & 22.3 \\
\hline Construction (inc. MEL) & 12.4 & 22.7 & 35.6 & 23.1 & 20.0 & 20.0 & 19.0 & 19.0 \\
\hline Total & 213.3 & 238.3 & 232.2 & 227.1 & 223.8 & 223.4 & 223.8 & 225.7 \\
\hline Work for Others & 45.3 & 48.1 & 58.0 & 58.1 & 61.2 & 63.3 & 63.7 & 64.9 \\
\hline Total Lab Funding & 258.6 & 286.4 & 290.2 & 285.2 & 285.0 & 286.7 & 287.5 & 290.6 \\
\hline
\end{tabular}


Personnel By Assistant Secretary Level Office (Fiscal Year FTE)

\begin{tabular}{lrrrrrrrr}
\hline Office/Program & 1993 & 1994 & 1995 & 1996 & 1997 & 1998 & 1999 & 2000 \\
\hline Office of Energy Research & 1213 & 1147 & 1143 & 1159 & 1173 & 1186 & 1199 & 1213 \\
Energy Efficiency \& Renewable Energy & 156 & 164 & 163 & 161 & 161 & 161 & 161 & 161 \\
Fossil Energy & 17 & 16 & 16 & 16 & 16 & 16 & 16 & 16 \\
Civilian Waste Management & 26 & 26 & 27 & 27 & 27 & 27 & 27 & 27 \\
Environment, Health and Safety & 12 & 12 & 7 & 7 & 7 & 7 & 7 & 7 \\
Environmental Restoration & 57 & 65 & 78 & 79 & 78 & 67 & 64 & 64 \\
Administration \& Human Resource & 9 & 9 & 10 & 9 & 9 & 9 & 9 & 9 \\
Domestic and International Energy Policy & 12 & 12 & 2 & 2 & 2 & 2 & 2 & 2 \\
Other DOE Contractors & 88 & 76 & 60 & 60 & 60 & 60 & 60 & 60 \\
$\quad$ Total DOE & $\mathbf{1 5 9 0}$ & $\mathbf{1 5 2 7}$ & $\mathbf{1 5 0 5}$ & $\mathbf{1 5 2 0}$ & $\mathbf{1 5 3 2}$ & $\mathbf{1 5 3 5}$ & $\mathbf{1 5 4 5}$ & $\mathbf{1 5 5 9}$ \\
Work for Others & 338 & $\mathbf{3 2 8}$ & $\mathbf{3 2 5}$ & 339 & 349 & 354 & $\mathbf{3 5 4}$ & 354 \\
$\quad$ Total Direct & $\mathbf{1 9 2 8}$ & $\mathbf{1 8 5 5}$ & $\mathbf{1 8 3 0}$ & $\mathbf{1 8 5 8}$ & $\mathbf{1 8 8 1}$ & $\mathbf{1 8 8 8}$ & $\mathbf{1 8 9 9}$ & $\mathbf{1 9 1 3}$ \\
$\quad$ Total Indirect & $\mathbf{7 8 7}$ & $\mathbf{7 7 6}$ & $\mathbf{7 6 1}$ & $\mathbf{7 6 4}$ & $\mathbf{7 7 0}$ & $\mathbf{7 7 0}$ & $\mathbf{7 7 0}$ & $\mathbf{7 7 1}$ \\
$\quad$ Total Personnel & $\mathbf{2 7 1 5}$ & $\mathbf{2 6 3 1}$ & $\mathbf{2 5 9 1}$ & $\mathbf{2 6 2 2}$ & $\mathbf{2 6 5 1}$ & $\mathbf{2 6 5 8}$ & $\mathbf{2 6 6 9}$ & $\mathbf{2 6 8 4}$ \\
\hline
\end{tabular}


Office of Energy Research Funding and Personnel (FY BA, \$M)

\begin{tabular}{|c|c|c|c|c|c|c|c|c|}
\hline Office/Program & 1993 & 1994 & 1995 & 1996 & 1997 & 1998 & 1999 & 2000 \\
\hline \multicolumn{9}{|l|}{ AT Fusion Energy } \\
\hline Operating & 7.3 & 3.8 & 5.3 & 5.5 & 5.5 & 5.5 & 5.5 & 5.5 \\
\hline Capital Equipment & 1.4 & 0.7 & 0.8 & 0.6 & 0.6 & 0.6 & 0.6 & 0.6 \\
\hline $\begin{array}{l}\text { Total } \\
\text { Direct FTE }\end{array}$ & $\begin{array}{l}8.7 \\
70\end{array}$ & $\begin{array}{l}4.5 \\
39\end{array}$ & $\begin{array}{l}6.1 \\
53\end{array}$ & $\begin{array}{l}6.1 \\
53\end{array}$ & $\begin{array}{c}6.1 \\
53\end{array}$ & $\begin{array}{l}6.1 \\
53\end{array}$ & $\begin{array}{l}6.1 \\
53\end{array}$ & $\begin{array}{l}6.1 \\
53\end{array}$ \\
\hline \multicolumn{9}{|c|}{ KA High Energy Physics } \\
\hline Operating & 19.8 & 19.6 & 19.4 & 20.0 & 20.3 & 20.6 & 20.9 & 21.2 \\
\hline Capital Equipment & 2.3 & 2.2 & 2.1 & 2.1 & 2.1 & 2.1 & 2.1 & 2.1 \\
\hline Construction & 0.0 & 2.8 & 0.0 & 0.0 & 0.0 & 0.0 & 0.0 & 0.0 \\
\hline $\begin{array}{l}\text { Total } \\
\text { Direct FTE }\end{array}$ & $\begin{array}{l}22.1 \\
167\end{array}$ & $\begin{array}{l}24.6 \\
164\end{array}$ & $\begin{array}{l}21.5 \\
159\end{array}$ & $\begin{array}{l}\mathbf{2 2 . 1} \\
157\end{array}$ & $\begin{array}{l}22.4 \\
160\end{array}$ & $\begin{array}{l}22.7 \\
162\end{array}$ & $\begin{array}{l}23.0 \\
165\end{array}$ & $\begin{array}{l}23.3 \\
167\end{array}$ \\
\hline \multicolumn{9}{|l|}{ KB Nuclear Physics } \\
\hline Operating & 25.5 & 22.2 & 17.4 & 18.2 & 18.2 & 18.2 & 18.2 & 18.2 \\
\hline Capital Equipment & 7.1 & 8.4 & 7.1 & 7.7 & 8.5 & 8.5 & 8.5 & 8.5 \\
\hline Construction & 3.7 & 3.6 & 4.2 & 4.9 & 5.0 & 5.0 & 5.0 & 5.0 \\
\hline Total & 36.3 & 34.2 & 28.7 & 30.8 & 31.7 & 31.7 & 31.7 & 31.7 \\
\hline Direct FTE & 272 & 234 & 215 & 222 & 222 & 222 & 222 & 222 \\
\hline \multicolumn{9}{|c|}{ KC 02 Materials Sciences } \\
\hline Operating & 38.5 & 37.9 & 36.0 & 37.0 & 37.6 & 38.2 & 38.7 & 39.3 \\
\hline Capital Equipment & 4.1 & 5.3 & 4.4 & 4.5 & 4.5 & 4.5 & 4.5 & 4.5 \\
\hline Construction & 1.6 & 1.8 & 2.5 & 3.0 & 3.0 & 3.0 & 3.0 & 3.0 \\
\hline Total & 44.2 & 45.0 & 42.9 & 44.5 & 45.1 & 45.7 & 46.2 & 46.8 \\
\hline Direct FTE & 357 & 355 & 343 & 339 & 344 & 349 & 355 & 360 \\
\hline \multicolumn{9}{|c|}{ KC 03 Chemical Sciences } \\
\hline Operating & 8.3 & 8.7 & 7.7 & 7.9 & 8.1 & 8.2 & 8.3 & 8.5 \\
\hline Capital Equipment & 2.3 & 2.5 & 1.0 & 1.0 & 1.0 & 1.0 & 1.0 & 1.0 \\
\hline $\begin{array}{l}\text { Total } \\
\text { Direct FTE }\end{array}$ & $\begin{array}{l}10.6 \\
69\end{array}$ & $\begin{array}{l}11.2 \\
64\end{array}$ & $\begin{array}{r}8.7 \\
61\end{array}$ & $\begin{array}{l}8.9 \\
60\end{array}$ & $\begin{array}{l}9.1 \\
61\end{array}$ & $\begin{array}{l}9.2 \\
62\end{array}$ & $\begin{array}{l}9.3 \\
63\end{array}$ & $\begin{array}{c}9.5 \\
64\end{array}$ \\
\hline \multicolumn{9}{|c|}{ KC 04 Engineering, Math and Geosciences } \\
\hline Operating & 2.5 & 2.4 & 1.6 & 1.6 & 1.7 & 1.7 & 1.7 & 1.7 \\
\hline Capital Equipment & 0.3 & 0.3 & 0.1 & 0.1 & 0.1 & 0.1 & 0.1 & 0.1 \\
\hline Total & 2.8 & 2.7 & 1.7 & 1.7 & 1.8 & 1.8 & 1.8 & 1.8 \\
\hline Direct FTE & 25 & 24 & 19 & 19 & 19 & 19 & 20 & 20 \\
\hline \multicolumn{9}{|c|}{ KC 05 Advanced Energy Projects } \\
\hline Operating & 0.5 & 0.5 & 0.5 & 0.5 & 0.5 & 0.5 & 0.5 & 0.5 \\
\hline Capital Equipment & 0.1 & 0.1 & 0.1 & 0.1 & 0.1 & 0.1 & 0.1 & 0.1 \\
\hline Total & 0.6 & 0.6 & 0.6 & 0.6 & 0.6 & 0.6 & 0.6 & 0.6 \\
\hline Direct FTE & 8 & 8 & 8 & 8 & 8 & 8 & 8 & 8 \\
\hline \multicolumn{9}{|c|}{ KC 06 Energy Biosciences } \\
\hline Operating & 1.1 & 1.0 & 1.1 & 1.1 & 1.1 & 1.2 & 1.2 & 1.2 \\
\hline Capital Equipment & 0.1 & 0.1 & 0.1 & 0.1 & 0.1 & 0.1 & 0.1 & 0.1 \\
\hline Total & 1.2 & 1.1 & 1.2 & 1.2 & 1.2 & 1.3 & 1.3 & 1.3 \\
\hline Direct FTE & 8 & 8 & 8 & 8 & 8 & 8 & 9 & 9 \\
\hline
\end{tabular}


Office of Energy Research Funding and Personnel (FY BA, \$M) (continued)

\begin{tabular}{|c|c|c|c|c|c|c|c|c|}
\hline Office/Program & 1993 & 1994 & 1995 & 1996 & 1997 & 1998 & 1999 & 2000 \\
\hline \multicolumn{9}{|c|}{ KC 07 Applied Math Sciences } \\
\hline Operating & 3.5 & 7.8 & 3.8 & 4.3 & 4.7 & 5.0 & 5.3 & 5.6 \\
\hline Capital Equipment & 0.1 & 0.3 & 0.1 & 0.1 & 0.1 & 0.1 & 0.1 & 0.1 \\
\hline Total & 3.6 & 8.1 & 3.9 & 4.4 & 4.8 & 5.1 & 5.4 & 5.7 \\
\hline Direct FTE & 28 & 30 & 30 & 33 & 36 & 38 & 40 & 43 \\
\hline \multicolumn{9}{|c|}{ KC Basic Energy Sciences (Total) } \\
\hline Operating & 54.4 & 58.3 & 50.7 & 52.6 & 53.7 & 54.8 & 55.8 & 56.9 \\
\hline Capital Equipment & 7.0 & 8.6 & 5.8 & 5.9 & 5.9 & 5.9 & 5.9 & 5.9 \\
\hline Construction & 1.6 & 1.8 & 2.5 & 3.0 & 3.0 & 3.0 & 3.0 & 3.0 \\
\hline Total & 63.0 & 68.7 & 59.0 & 61.5 & 62.6 & 63.7 & 64.7 & 65.8 \\
\hline Direct FTE & 495 & 489 & 469 & 467 & 476 & 485 & 494 & 503 \\
\hline \multicolumn{9}{|c|}{ KG General Purpose Facilities Revitalization } \\
\hline Operating & 0.0 & 0.3 & 0.2 & 0.2 & 0.2 & 0.2 & 0.2 & 0.2 \\
\hline Capital Equipment & 0.0 & 0.1 & 0.0 & 0.0 & 0.0 & 0.0 & 0.0 & 0.0 \\
\hline Construction & 4.6 & 5.0 & 5.8 & 5.7 & 10.0 & 10.0 & 10.0 & 10.0 \\
\hline Total & 4.6 & 5.4 & 6.0 & 5.9 & 10.2 & 10.2 & 10.2 & 10.2 \\
\hline Direct FTE & 24 & 24 & 26 & 26 & 26 & 26 & 26 & 26 \\
\hline \multicolumn{9}{|c|}{ KP Biological and Environmental Research } \\
\hline Operating & 20.4 & 21.2 & 22.9 & 25.2 & 25.5 & 25.7 & 26.0 & 26.2 \\
\hline Capital Equipment & 1.9 & 2.6 & 1.1 & 1.1 & 1.1 & 1.1 & 1.1 & 1.1 \\
\hline Construction & 0.6 & 2.7 & 20.5 & 8.3 & 1.0 & 1.0 & 0.0 & 0.0 \\
\hline Total & 22.9 & 26.5 & 44.5 & 34.6 & 27.6 & 27.8 & 27.1 & 27.3 \\
\hline Direct FTE & 165 & 165 & 183 & 196 & 198 & 200 & 202 & 204 \\
\hline \multicolumn{9}{|c|}{ KS Superconducting Super Collider } \\
\hline Operating & 0.0 & 0.1 & 0.0 & 0.0 & 0.0 & 0.0 & 0.0 & 0.0 \\
\hline Capital Equipment & 0.4 & 0.0 & 0.0 & 0.0 & 0.0 & 0.0 & 0.0 & 0.0 \\
\hline Total & 0.4 & 0.1 & 0.0 & 0.0 & 0.0 & $\mathbf{0 . 0}$ & 0.0 & $\mathbf{0 . 0}$ \\
\hline Direct FTE & 6 & 4 & 0 & 0 & 0 & 0 & 0 & 0 \\
\hline \multicolumn{9}{|c|}{$\mathrm{KT} / \mathrm{KV}$ University and Science Education } \\
\hline Operating & 2.4 & 2.6 & 2.8 & 3.0 & 3.0 & 3.0 & 3.0 & 3.0 \\
\hline Total & 2.4 & 2.6 & 2.8 & 3.0 & 3.0 & 3.0 & 3.0 & 3.0 \\
\hline Direct FTE & 7 & 8 & 8 & 9 & 9 & 9 & 9 & 9 \\
\hline \multicolumn{9}{|c|}{ KU Laboratory Technology Transfer } \\
\hline Operating & 1.7 & 4.4 & 8.8 & 9.5 & 9.5 & 9.5 & 9.5 & 9.5 \\
\hline Total & 1.7 & 4.4 & 8.8 & 9.5 & 9.5 & 9.5 & 9.5 & 9.5 \\
\hline Direct FTE & 7 & 20 & 30 & 30 & 30 & 30 & 30 & 30 \\
\hline \multicolumn{9}{|l|}{ Total OER } \\
\hline Operating & 131.5 & 132.5 & 127.5 & 134.1 & 135.8 & 137.4 & 139.0 & 140.6 \\
\hline Capital Equipment & 20.1 & 22.6 & 16.9 & 17.4 & 18.2 & 18.2 & 18.2 & 18.2 \\
\hline Construction & 10.5 & 15.9 & 33.0 & 21.9 & 19.0 & 19.0 & 18.0 & 18.0 \\
\hline Total & 162.1 & 171.0 & 177.4 & 173.4 & 173.0 & 174.6 & 175.2 & 176.8 \\
\hline Direct FTE & 1213 & 1147 & 1143 & 1159 & 1173 & 1186 & 1199 & 1213 \\
\hline
\end{tabular}


Energy Efficiency and Renewable Energy Funding and Personnel (FY BA, \$M)

\begin{tabular}{|c|c|c|c|c|c|c|c|c|}
\hline Office/Program & 1993 & 1994 & 1995 & 1996 & 1997 & 1998 & 1999 & 2000 \\
\hline \multicolumn{9}{|c|}{ AK Electric Energy Systems } \\
\hline Operating & 0.2 & 0.3 & 0.2 & 0.2 & 0.2 & 0.2 & 0.2 & 0.2 \\
\hline Total & 0.2 & 0.3 & 0.2 & 0.2 & 0.2 & 0.2 & 0.2 & 0.2 \\
\hline Direct FTE & 1 & 1 & 1 & 1 & 1 & 1 & 1 & 1 \\
\hline \multicolumn{9}{|l|}{ AM Geothermal Energy } \\
\hline Operating & 0.8 & 0.0 & 0.0 & 0.0 & 0.0 & 0.0 & 0.0 & 0.0 \\
\hline Total & 0.8 & 0.0 & 0.0 & $\mathbf{0 . 0}$ & 0.0 & 0.0 & 0.0 & 0.0 \\
\hline Direct FTE & 7 & 1 & 0 & 0 & 0 & 0 & 0 & 0 \\
\hline \multicolumn{9}{|l|}{ EC Building Sector } \\
\hline Operating & 10.6 & 16.4 & 14.4 & 14.8 & 14.8 & 14.8 & 14.8 & 14.8 \\
\hline Capital Equipment & 0.5 & 1.0 & 0.6 & 0.6 & 0.6 & 0.6 & 0.6 & 0.6 \\
\hline Total & 11.1 & 17.4 & 15.0 & 15.4 & 15.4 & 15.4 & 15.4 & 15.4 \\
\hline Direct FTE & 104 & 120 & 120 & 119 & 119 & 119 & 119 & 119 \\
\hline \multicolumn{9}{|l|}{ ED Industrial Sector } \\
\hline Operating & 1.0 & 1.0 & 0.9 & 0.9 & 0.9 & 0.9 & 0.9 & 0.9 \\
\hline Capital Equipment & 0.1 & 0.0 & 0.0 & 0.0 & 0.0 & 0.0 & 0.0 & 0.0 \\
\hline Total & 1.1 & 1.0 & 0.9 & 0.9 & 0.9 & 0.9 & 0.9 & 0.9 \\
\hline Direct FTE & 10 & 9 & 8 & 8 & 8 & 8 & 8 & 8 \\
\hline \multicolumn{9}{|c|}{ EE Transportation Sector } \\
\hline Operating & 4.0 & 5.4 & 3.8 & 3.9 & 3.9 & 3.9 & 3.9 & 3.9 \\
\hline Capital Equipment & 0.1 & 0.3 & 0.1 & 0.1 & 0.1 & 0.1 & 0.1 & 0.1 \\
\hline Total & 4.1 & 5.7 & 3.9 & 4.0 & 4.0 & 4.0 & 4.0 & 4.0 \\
\hline Direct FTE & 23 & 23 & 23 & 23 & 23 & 23 & 23 & 23 \\
\hline \multicolumn{9}{|l|}{ EK Utility Sector } \\
\hline Operating & 1.0 & 1.3 & 1.1 & 1.1 & 1.1 & 1.1 & 1.1 & 1.1 \\
\hline Total & 1.0 & 1.3 & 1.1 & 1.1 & 1.1 & 1.1 & 1.1 & 1.1 \\
\hline Direct FTE & 11 & 10 & 11 & 11 & 11 & 11 & 11 & 11 \\
\hline \multicolumn{9}{|c|}{ Total Energy Efficiency and Renewable Energy } \\
\hline Operating & 17.6 & 24.6 & 20.4 & 21.0 & 21.0 & 21.0 & 21.0 & 21.0 \\
\hline Capital Equipment & 0.7 & 1.3 & 0.7 & 0.7 & 0.7 & 0.7 & 0.7 & 0.7 \\
\hline Total & 18.3 & 25.9 & 21.1 & 21.7 & 21.7 & 21.7 & 21.7 & 21.7 \\
\hline Direct FTE & 156 & 164 & 163 & 161 & 161 & 161 & 161 & 161 \\
\hline
\end{tabular}


Fossil Fuel and Other DOE Program Funding and Personnel (FY BA, \$M)

\begin{tabular}{|c|c|c|c|c|c|c|c|c|}
\hline Office/Program & 1993 & 1994 & 1995 & 1996 & 1997 & 1998 & 1999 & 2000 \\
\hline \multicolumn{9}{|l|}{ AA Coal } \\
\hline Operating & 0.9 & 0.9 & 0.7 & 0.7 & 0.7 & 0.7 & 0.7 & 0.7 \\
\hline Capital Equipment & 0.0 & 0.0 & 0.1 & 0.0 & 0.1 & 0.0 & 0.1 & 0.0 \\
\hline Total & 0.9 & 0.9 & 0.8 & 0.7 & 0.8 & 0.7 & 0.8 & 0.7 \\
\hline Direct FTE & 6 & 5 & 4 & 4 & 4 & 4 & 4 & 4 \\
\hline \multicolumn{9}{|l|}{ AB Gas } \\
\hline Operating & 0.5 & 0.5 & 0.5 & 0.5 & 0.5 & 0.5 & 0.5 & 0.5 \\
\hline Total 0 & 0.5 & 0.5 & 0.5 & 0.5 & 0.5 & 0.5 & 0.5 & 0.5 \\
\hline Direct FTE & 4 & 4 & 4 & 4 & 4 & 4 & 4 & 4 \\
\hline \multicolumn{9}{|l|}{ AC Petroleum } \\
\hline Operating & 0.8 & 1.9 & 0.8 & 0.8 & 0.8 & 0.8 & 0.8 & 0.8 \\
\hline Total & 0.8 & 1.9 & 0.8 & 0.8 & 0.8 & 0.8 & 0.8 & 0.8 \\
\hline Direct FTE & 7 & 7 & 7 & 7 & 7 & 7 & 7 & 7 \\
\hline \multicolumn{9}{|l|}{ Total Fossil } \\
\hline Operating & 2.2 & 3.3 & 2.0 & 2.1 & 2.1 & 2.1 & 2.1 & 2.1 \\
\hline Capital Equipment & 0.0 & 0.0 & 0.1 & 0.0 & 0.1 & 0.0 & 0.1 & 0.0 \\
\hline Total & 2.2 & 3.3 & 2.1 & 2.1 & 2.2 & 2.1 & 2.2 & 2.1 \\
\hline Direct FTE & 17 & 16 & 16 & 16 & 16 & 16 & 16 & 16 \\
\hline \multicolumn{9}{|c|}{ DB Civilian Waste Management } \\
\hline Operating & 3.3 & 3.8 & 3.5 & 3.6 & 3.6 & 3.6 & 3.6 & 3.6 \\
\hline Capital Equipment & 0.1 & 0.2 & 0.1 & 0.1 & 0.1 & 0.1 & 0.1 & 0.1 \\
\hline Total & 3.4 & 4.0 & 3.6 & 3.7 & 3.7 & 3.7 & 3.7 & 3.7 \\
\hline Direct FTE & 26 & 26 & 27 & 27 & 27 & 27 & 27 & 27 \\
\hline \multicolumn{9}{|c|}{ EX/EW Environmental Restoration and Waste Management } \\
\hline Operating & 10.1 & 12.5 & 15.3 & 17.2 & 14.4 & 12.5 & 12.2 & 12.5 \\
\hline Capital Equipment & 0.2 & 0.9 & 0.6 & 0.2 & 0.2 & 0.2 & 0.2 & 0.2 \\
\hline Construction & 0.5 & 5.7 & 0.6 & 0.2 & 0.0 & 0.0 & 0.0 & 0.0 \\
\hline Total & 10.8 & 19.1 & 16.5 & 17.6 & 14.6 & 12.7 & 12.4 & 12.7 \\
\hline Direct FTE & 57 & 65 & 78 & 79 & 78 & 67 & 64 & 64 \\
\hline \multicolumn{9}{|c|}{ HA Environment, Safety \& Health } \\
\hline Operating & 2.6 & 1.7 & 1.0 & 1.0 & 1.0 & 1.1 & 1.1 & 1.1 \\
\hline Total & 2.6 & 1.7 & 1.0 & 1.0 & 1.0 & 1.1 & 1.1 & 1.1 \\
\hline Direct FTE & 12 & 12 & 7 & 7 & 7 & 7 & 7 & 7 \\
\hline \multicolumn{9}{|c|}{ WB Administration and Human Resource Management } \\
\hline Operating & 0.2 & 0.7 & 0.3 & 0.3 & 0.3 & 0.3 & 0.3 & 0.3 \\
\hline Construction & 1.4 & 1.1 & 2.0 & 1.0 & 1.0 & 1.0 & 1.0 & 1.0 \\
\hline Total & 1.6 & 1.8 & 2.3 & 1.3 & 1.3 & 1.3 & 1.3 & 1.3 \\
\hline Direct FTE & 9 & 9 & 9 & 9 & 9 & 9 & 9 & 9 \\
\hline \multicolumn{9}{|c|}{ PE Domestic and International Energy Policy } \\
\hline Operating & 0.8 & 0.5 & 0.2 & 0.2 & 0.2 & 0.2 & 0.2 & 0.2 \\
\hline Total & 0.8 & 0.5 & 0.2 & 0.2 & 0.2 & 0.2 & 0.2 & 0.2 \\
\hline Direct FTE & 12 & 12 & 2 & 2 & 2 & 2 & 2 & 2 \\
\hline \multicolumn{9}{|c|}{ Work for Other DOE Contractors } \\
\hline Operating & 5.5 & 4.7 & 3.0 & 3.0 & 3.0 & 3.0 & 3.0 & 3.0 \\
\hline Capital Equipment & 6.0 & 6.3 & 5.0 & 3.1 & 3.1 & 3.1 & 3.1 & 3.1 \\
\hline Total & 11.5 & 11.0 & 8.0 & 6.1 & 6.1 & 6.1 & 6.1 & 6.1 \\
\hline Direct FTE & 88 & 76 & 60 & 60 & 60 & 60 & 60 & 60 \\
\hline
\end{tabular}


Work for Others Funding and Personnel (FY BA, \$M)

\begin{tabular}{|c|c|c|c|c|c|c|c|c|}
\hline Office/Program & 1993 & 1994 & 1995 & 1996 & 1997 & 1998 & 1999 & 2000 \\
\hline \multicolumn{9}{|c|}{ Other Federal Agencies } \\
\hline AID & 0.4 & 0.4 & 0.5 & 0.5 & 0.5 & 0.5 & 0.5 & 0.5 \\
\hline Direct FTE & 3 & 3 & 3 & 3 & 3 & 3 & 3 & 3 \\
\hline Defense & 7.5 & 9.0 & 19.0 & 15.0 & 15.0 & 15.0 & 15.0 & 15.0 \\
\hline Direct FTE & 51 & 58 & 55 & 55 & 55 & 55 & 55 & 55 \\
\hline EPA & 1.6 & 1.2 & 1.2 & 1.2 & 1.2 & 1.2 & 1.2 & 1.2 \\
\hline Direct FTE & 12 & 11 & 11 & 11 & 11 & 11 & 11 & 11 \\
\hline DOI & 0.6 & 0.5 & 0.7 & 0.7 & 0.7 & 0.7 & 0.7 & 0.7 \\
\hline Direct FTE & 5 & 4 & 4 & 4 & 4 & 4 & 4 & 4 \\
\hline NASA & 2.7 & 2.7 & 2.5 & 2.5 & 2.6 & 2.6 & 2.6 & 2.6 \\
\hline Direct FTE & 22 & 17 & 22 & 22 & 22 & 22 & 22 & 22 \\
\hline $\mathrm{NIH}$ & 13.7 & 14.8 & 15.9 & 20.0 & 23.0 & 25.0 & 25.0 & 26.0 \\
\hline Direct FTE & 109 & 103 & 116 & 130 & 140 & 145 & 145 & 145 \\
\hline Other & 0.4 & 0.4 & 1.2 & 1.2 & 1.2 & 1.3 & 1.7 & 1.9 \\
\hline Direct FTE & 2 & 4 & 6 & 6 & 6 & 6 & 6 & 6 \\
\hline \multicolumn{9}{|c|}{ Total Other Federal Agencies } \\
\hline Operating & 24.5 & 25.0 & 28.4 & 28.8 & 38.7 & 42.1 & 42.5 & 43.7 \\
\hline Capital Equipment & 2.4 & 4.0 & 12.6 & 12.3 & 5.5 & 4.2 & 4.2 & 4.2 \\
\hline Total & 26.9 & 29.0 & 41.0 & 41.1 & 44.2 & 46.3 & 46.7 & 47.9 \\
\hline Direct FTE & 204 & 200 & 217 & 231 & 241 & 246 & 246 & 246 \\
\hline \multicolumn{9}{|l|}{ State/Private } \\
\hline Operating & 16.1 & 18.0 & 16.0 & 16.0 & 16.0 & 16.0 & 16.0 & 16.0 \\
\hline Capital Equipment & 2.3 & 1.1 & 1.0 & 1.0 & 1.0 & 1.0 & 1.0 & 1.0 \\
\hline Total & 18.4 & 19.1 & 17.0 & 17.0 & 17.0 & 17.0 & 17.0 & 17.0 \\
\hline Direct FTE & 134 & 128 & 108 & 108 & 108 & 108 & 108 & 108 \\
\hline \multicolumn{9}{|l|}{ Total Work for Others } \\
\hline Operating & 40.6 & 43.0 & 44.4 & 44.8 & 54.7 & 58.1 & 58.5 & 59.7 \\
\hline Capital Equipment & 4.7 & 5.1 & 13.6 & 13.3 & 6.5 & 5.2 & 5.2 & 5.2 \\
\hline Total & 45.3 & 48.1 & $\mathbf{5 8 . 0}$ & 58.1 & 61.2 & 63.3 & 63.7 & 64.9 \\
\hline Direct FTE & 338 & 328 & 325 & 339 & 349 & 354 & 354 & 354 \\
\hline
\end{tabular}


Subcontracting and Procurement (\$M)

\begin{tabular}{lcccc}
\hline Recipient & FY 1992 & FY 1993 & FY 1994 (Projected) & FY 1995 (Projected) \\
\hline Universities & 10.0 & 8.0 & 7.5 & 8.0 \\
All Other & 80.0 & 69.0 & 66.6 & 75.0 \\
Other DOE & 2.0 & 1.0 & 1.5 & 1.0 \\
Total & $\mathbf{9 2 . 0}$ & $\mathbf{7 8 . 0}$ & $\mathbf{7 5 . 6}$ & $\mathbf{8 4 . 0}$ \\
\hline
\end{tabular}

Procurement from Disadvantaged, Women Owned, and All Small Businesses

\begin{tabular}{lccc}
\hline Business & FY 1992 & FY 1993 & FY 1994 \\
Category & $\$ M(\%)$ & $\$ M(\%)$ & $\$ M(\%)$ \\
\hline Disadvantaged & $6.4(8)$ & $7.0(9)$ & $9.6(12.7)$ \\
Women Owned & $4.0(5)$ & $3.9(5)$ & $5.1(6.6)$ \\
All Small & $36.0(45)$ & $35.8(46)$ & $44.5(52.9)$ \\
\hline
\end{tabular}




\section{$V I I$}

ACKNOWLEDGMENTS

Institutional planning at LBL is conducted as an annual management activity based on technical information contributed by the Laboratory's Division Directors (see organization chart, Section II). Preparation of reporting documents is coordinated through the Office for Planning and Communications. Divisional staff coordinating information and assisting in preparation include:

Accelerator and Fusion Research

Chemical Sciences

Earth Sciences

Energy and Environment

Engineering

Environment, Health and Safety

Information and Computing Sciences

Life Sciences

Materials Sciences

Nuclear Science

Physics

Structural Biology
Christine Celata, Joseph Chew, Richard Gough, Henry Rutkowski Linda Maio Ellen Klahn, Karl Olson Donald Grether Mary Clary John Bartley, Mark Kotowski David Stevens Mohandas Narla, David Gilbert Mark Alper Janis Dairiki Pauline Fong James Bartholomew

Robert Johnson, Cheryl McFate, and Douglas Vaughn, of the Deputy Director's Office, provided review and comments, and scientific program leaders contributed to specific sections of the plan through division offices. In addition, elements of the document are developed in conjunction with 
responsible support program administrators and staff:

Assessment and Assurance

Budget and Resource Planning

Educational Programs

Facilities Planning

Laboratory Directed R\&D

Technology Transfer

Workforce Diversity
Jon Stanley

Patricia Jenkins, Benjamin Shuey Roland Otto, Eileen Kraskouskas

Laura Chen Todd Hansen

Bruce Davies Christine Jue

Correspondence regarding the Institutional Plan can be directed to:

Michael A. Chartock

Office for Planning and Communications

Lawrence Berkeley Laboratory, MS 50A-4112

University of California

Berkeley, California 94720

(415) 486-6669; (Fax) 486-6866

MAChartock@lbl.gov 


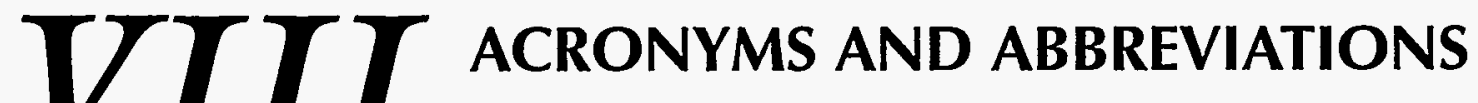

$\begin{array}{ll} & \\ \text { ACET } & \text { Alameda Center for Environmental Technologies } \\ \text { ACTI } & \text { Advanced Computational Technology Initiative } \\ \text { ADEPT } & \text { Assisting Development of Energy Practices and Technology } \\ \text { AECR } & \text { Advanced Electron Cyclotron Resonance } \\ \text { AGMUS } & \text { Ana G. Méndez University System } \\ \text { AID } & \text { Agency for international Development } \\ \text { ALS } & \text { Advanced Light Source } \\ \text { AMTEX } & \text { AMerican TEXtile Partnership } \\ \text { ARPA } & \text { Advanced Research Projects Agency } \\ \text { ASC } & \text { Alameda Science City } \\ \text { ATLAS } & \text { A Toroidal LHC Apparatus } \\ \text { BA } & \text { Budget Authority } \\ \text { BASTEC } & \text { Bay Area Science and Technology Education Collaboration } \\ \text { BES } & \text { Basic Energy Sciences (Office) } \\ \text { BESAC } & \text { Basic Energy Sciences Advisory Committee } \\ \text { BNL } & \text { Brookhaven National Laboratory } \\ \text { CEE } & \text { California Environmental Enterprise } \\ \text { CAM } & \text { Center for Advanced Materials } \\ \text { CAMP } & \text { Capital Asset Management Plan } \\ \text { CAS } & \text { Condition Assessment Survey } \\ \text { CCAP } & \text { Climate Change Action Plan } \\ \text { CDF } & \text { Collider Detector Facility at Fermilab } \\ \text { CDRL } & \text { Chemical Dynamics Research Laboratory } \\ \text { CEDR } & \text { Comprehensive Epidemiologic Data Resource } \\ \text { CEQA } & \text { California Environmental Quality Act } \\ \text { CERN } & \text { European Organization for Nuclear Research } \\ \text { CIEE } & \text { California Institute for Energy Efficiency } \\ \text { CJO } & \text { Current Job Opportunities (Bulletin) } \\ & \end{array}$




\begin{tabular}{|c|c|}
\hline$C P$ & charge parity \\
\hline CFC & chlorofluorocarbon \\
\hline CRADAs & Cooperative Research and Development Agreements \\
\hline CSEE & Center for Science and Engineering Education \\
\hline $\mathrm{CXRO}$ & Center for X-ray Optics \\
\hline$D \& D$ & Decontamination and Decommissioning \\
\hline DAMA & Demand Activated Manufacturing Architecture \\
\hline DBMS & data base management system \\
\hline DEEP & data base on energy-efficiency programs \\
\hline DNA & deoxyribonucleic acid \\
\hline DOD & U.S. Department of Defense \\
\hline DOE & U.S. Department of Energy \\
\hline DOE/EM & $\begin{array}{l}\text { U.S. Department of Energy, Office of Environmental } \\
\text { Research }\end{array}$ \\
\hline DOE/ER & U.S. Department of Energy, Office of Energy Research \\
\hline DOE/OAK & DOE Operations Office, Oakland \\
\hline DOI & U.S. Department of Interior \\
\hline DP & Office of Defense Programs \\
\hline EAP & Employee Assistance Program \\
\hline EBMF & Electron Beam Microcharacterization Facility \\
\hline ECC & Emergency Command Center \\
\hline ECM & extracellular matrix \\
\hline EEO & equal employment opportunity \\
\hline EH\&S & Environment, Health and Safety Division (LBL) \\
\hline EM & Environmental Management \\
\hline EPA & U.S. Erivironmental Protection Agency \\
\hline EPRI & Electric Power Research Institute \\
\hline ER & Office of Energy Research (see also OER) \\
\hline ER-LTT & DOE Energy Research Laboratory Technology Transfer \\
\hline ERWM & Environmental Restoration and Waste Management \\
\hline ES\&H & environment, safety, and health \\
\hline ESnet & Energy Sciences Network \\
\hline EUV & extreme ultraviolet \\
\hline FE & Office of Fossil Energy \\
\hline FEMP & Federal Energy Management Program \\
\hline FNAL & Fermilab National Accelerator Laboratory \\
\hline FTE & full-time equivalent \\
\hline FY & fiscal year \\
\hline GPE & General Purpose Equipment \\
\hline GPO & Government Printing Office \\
\hline GPP & General Plant Projects \\
\hline HEPA & high-efficiency particulate air \\
\hline HIFAR & Heavy-Ion Fusion Accelerator Research \\
\hline HGC & Human Genome Center \\
\hline HVAC & heating, ventilating, and air-conditioning \\
\hline HWHF & Hazardous Waste Handling Facility \\
\hline ILSE & Induction Linac Systems Experiments \\
\hline IRM & information resources management \\
\hline ISL & IsoSpin Laboratory \\
\hline ITD & Institute for Technology Development \\
\hline JAERI & Japan Atomic Energy Research Institute \\
\hline JSU & Jackson State University \\
\hline LANL & Los Alamos National Laboratory \\
\hline
\end{tabular}




\begin{tabular}{|c|c|}
\hline LBL & Lawrence Berkeley Laboratory \\
\hline LDRD & Laboratory Directed Research and Development Program \\
\hline LEAP & Leadership Education for Asian Pacifics \\
\hline LHC & Large Hadron Collider \\
\hline LIGA & Lithographie Galvanoformung, Abformung \\
\hline LIMS & Laboratory Information Management System \\
\hline LLNL & Lawrence Livermore National Laboratory \\
\hline M\&F & male and female \\
\hline $\mathrm{M} \& \mathrm{O}$ & maintenance and operations \\
\hline MEL-FS & Multiprogram Energy Laboratory Facilities Support \\
\hline MEMS & microelectromechanical systems \\
\hline NASA & National Aeronautics and Space Administration \\
\hline NCEM & National Center for Electron Microscopy \\
\hline NEPA & National Environmental Policy Act \\
\hline NIH & National Institutes of Health \\
\hline NII & National Information Infrastructure \\
\hline NMR & nuclear magnetic resonance \\
\hline NSCORT & NASA Specialized Center for Research and Training \\
\hline NSF & National Science Foundation \\
\hline OAP & Operating and Assurance Program \\
\hline OER & Office of Energy Research (see also ER) \\
\hline OER/DNP & Office of Energy Research, Division of Nuclear Physics \\
\hline OHER & Office of Health and Environmental Research \\
\hline OSHA & Occupational Safety and Health Administration \\
\hline OPA & Outstanding Performance Award \\
\hline PC & personal computer \\
\hline PEP & Positron-Electron Project \\
\hline PNGV & Partnership for a New Generation of Vehicles \\
\hline R\&D & research and development \\
\hline RHIC & Relativistic Heavy-lon Collider \\
\hline RNA & ribonucleic acid \\
\hline RPM & Risk Prioritization Matrix \\
\hline SDC & Solenoidal Detector Collaboration \\
\hline SDP & Site Development Plan \\
\hline SFI & Strategic Facilities Initiative \\
\hline SLAC & Stanford Linear Accelerator Center \\
\hline SLC & Stanford Linear Collider \\
\hline SNL & Sandia National Laboratories \\
\hline SNO & Sudbury Neutrino Observatory \\
\hline SPS & Super Proton Synchrotron \\
\hline SSC & Superconducting Super Collider \\
\hline STAR & Solenoidal Tracker at RHIC \\
\hline SXR & soft x-ray \\
\hline TEC & total estimated cost \\
\hline TPC & Time Projection Chamber \\
\hline UC & University of California \\
\hline UCOP & University of California, Office of the President \\
\hline VAT & Video-Audio Conferencing Tool \\
\hline VOC & volatile organic compound \\
\hline VUV & vacuum ultraviolet \\
\hline WFO & work for others \\
\hline YAC & yeast artificial chromosome \\
\hline
\end{tabular}

